\title{
EFFECTS OF HIGH INTENSITY, LARGE-SCALE FREESTREAM COMbustor TurbulenCe ON Heat Transfer In TransoniC TURBINE BLADES
}

\author{
by \\ Andrew Carl Nix, MS \\ Dissertation submitted to the Faculty of the \\ Virginia Polytechnic Institute and State University \\ in partial fulfillment of the requirements for the degree of \\ Doctor of Philosophy \\ in \\ Mechanical Engineering
}

\author{
APPROVED: \\ Dr. Thomas E. Diller, Co-Chairman \\ Dr. Wing F. Ng, Co-Chairman \\ Dr. Karen A. Thole \\ Dr. Joseph A. Schetz \\ Dr. Clinton L. Dancey
}

April, 2003

Blacksburg, Virginia

Keywords: Heat Transfer, Turbine, Freestream Turbulence, Transonic Cascade Copyright (C2003, Andrew C. Nix 


\title{
EFFECTS OF High INTENSITY, LARGE-SCALE FREESTREAM COMBUSTOR TURBULENCE ON HEAT TRANSFER IN TRANSONIC TURBINE BLADES
}

\author{
Andrew Carl Nix \\ Virginia Polytechnic Institute and State University, 2003 \\ Advisors: Dr. Thomas E. Diller and Dr. Wing F. Ng
}

(ABSTRACT)

The influence of freestream turbulence representative of the flow downstream of a modern gas turbine combustor and first stage vane on turbine blade heat transfer has been measured and analytically modeled in a linear, transonic turbine cascade. Measurements were performed on a high turning, transonic turbine blade. The facility is capable of heated flow with inlet total temperature of $120^{\circ} \mathrm{C}$ and inlet total pressure of $10 \mathrm{psig}$. The Reynolds number based on blade chord and exit conditions $\left(5 \times 10^{6}\right)$ and the inlet and exit Mach numbers (0.4 and 1.2, respectively) are representative of conditions in a modern gas turbine engine. High intensity, large length-scale freestream turbulence was generated using a passive turbulence-generating grid to simulate the turbulence generated in modern combustors after it has passed through the first stage vane row. The grid produced freestream turbulence with intensity of approximately $10-12 \%$ and an integral length scale of $2 \mathrm{~cm}$ near the entrance of the cascade passages, which is believed to be representative of the core flow entering a first stage gas turbine rotor blade row. Mean heat transfer results showed an increase in heat transfer coefficient of approximately $8 \%$ on the suction surface of the blade, with increases on the pressure surface on the order of two times higher than on the suction surface (approximately 17\%). This corresponds to increases in blade surface temperature of $5-10 \%$, which can significantly reduce the life of a turbine blade. The heat transfer data were compared with correlations from published literature with good agreement. 
Time-resolved surface heat transfer and passage velocity measurements were performed to investigate and quantify the effects of the turbulence on heat transfer and to correlate velocity fluctuations with heat transfer fluctuations. The data demonstrates strong coherence in velocity and heat flux at a frequency correlating with the most energetic eddies in the turbulence flow field (the integral length-scale). An analytical model was developed to predict increases in surface heat transfer due to freestream turbulence based on local measurements of turbulent velocity fluctuations ( $u_{\text {RMS }}^{\prime}$ ) and length-scale $\left(\Lambda_{\mathrm{x}}\right)$. The model was shown to predict measured increases in heat flux on both blade surfaces in the current data. The model also successfully predicted the increases in heat transfer measured in other work in the literature, encompassing different geometries (flat plate, cylinder, turbine vane and turbine blade) as well as both laminar and turbulent boundary layers, but demonstrated limitations in predicting early transition and heat transfer in turbulent boundary layers. Model analyses in the frequency domain provided valuable insight into the scales of turbulence that are most effective at increasing surface heat transfer. 


\section{ACKNOWLEDGMENTS}

This work was supported by the Air Force Office of Scientific Research (AFOSR) under grant number F49620-01-1-0177, monitored by Dr. Tom Beutner. I would like to thank Paul Giel, Bob Boyle and Jim Van Fossen at NASA Glenn for their help and insight into developing and evaluating the turbulence generator.

I would like to thank the members of my committee for their help, guidance, insight and support throughout my studies. Many of my committee members I have known for 8 years now, through my Master's work and my Ph.D. Dr. Diller has helped me stay focused on the task at hand and has provided me with a wealth of knowledge of heat transfer and the ability to take a step back and look at things from a different perspective. He also was there to give me a push when I was starting to lose steam. Dr. $\mathrm{Ng}$ has provided me with valuable insight into many aspects of my work and also has helped me prepare for a future career in academia. Dr. Thole has helped me with her knowledge of turbulence and turbulent heat transfer, as well as with writing and editing research papers and preparing presentations. Dr. Schetz and Dr. Dancey have always been there to answer questions on compressible flow. Most of my knowledge of boundary layers and high-speed compressible flow were taught to me by them. I would also like to thank Dr. Al Wicks for his help with signal processing. Dr. Wicks was always willing to sit down and discuss data acquisition and signal processing (as well as golf).

The support and friendship of my fellow graduate students is greatly appreciated, especially Austin Smith. Austin's help with data collection and the day-to-day "grunt work" in the tunnel, as well as out-of-lab diversions helped me get through some difficult 
times. Thanks also to Todd Bailie, Bo Song, Jason Anderson and Angie Rabe for their help and advice and general conversation throughout my studies, and to the graduate students of Dr. Thole, especially Andy Lethander and Mike Barringer.

Thanks to the guys in the Mechanical Engineering and the Aerospace and Ocean Engineering machine shops, Johnny Cox, Bill Songer, Tim Kessinger and Jamie Dowdy in the ME shop and Bruce Stanger and Mike Vaught in the AOE shop. Whenever I was in a bind with machining work or in need of parts for my experiments, the guys in the shop always came through for me. I would also like to thank the "new guy", Steve Edwards, in the AOE electronics shop. Steve quickly became an integral part of the dayto-day operations of the wind tunnel facilities and is a valuable asset to the $\mathrm{AOE}$ department and the wind tunnel facilities.

Finally, thank you to my wife and family. None of this would have happened without the support (both emotionally and financially) of my wife, Cheryl, and the encouragement of my family. It was not easy for us to leave a financially stable life with two professional jobs to allow me to return to school to pursue my Ph.D. The sacrifice Cheryl made to help me to achieve my academic goals is beyond words. One other family member has helped me immensely, my golden retriever, Seamus. When I was down or stressed out and after many all-nighters, all it took was that wagging tail and happy greeting from my faithful dog when I got home to cheer me up.

DREW NIX

Virginia Polytechnic Institute and State University April 2003 


\section{CONTENTS}

$\begin{array}{ll}\text { AbSTRACT } & \text { ii }\end{array}$

ACKNOWLEDGEMENTS iv

LIST OF TABLES $\quad$ ix

LIST OF FIGURES $\quad$ X

NOMENCLATURE

CHAPTER 1 INTRODUCTION 1

1.1 Turbine Blade Heat Transfer - Problem Statement 1

1.2 LiteratURE REVIEW 2

1.2.1 Turbulent Heat Transfer Studies and Correlations 2

1.2.1.1 Flat Plate Experiments 3

1.2.1.2 Cylinder Experiments 4

1.2.1.3 Turbine Vane and Blade Experiments 6

1.2.2 Turbulence Generation Methods 8

1.2.3 Time-Resolved Heat Transfer 8

$\begin{array}{ll}1.3 & \text { OVERVIEW OF CURRENT WORK } \\ \end{array}$

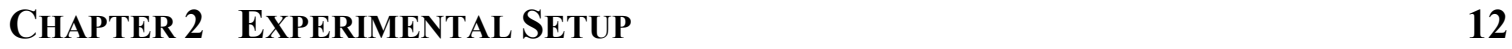

2.1 Transonic CASCAdE Wind TunNEL 12

2.2 Cascade Test Section and Blade Instrumentation 14

$\begin{array}{lll}2.3 & \text { INSTRUMENTATION AND DATA ACQUISITION SYSTEMS } & 18\end{array}$

2.3.1 Vatell HFM-7 Heat Flux Sensors 18

2.3.2 Kulite Pressure Transducers 20

2.3.3 Hot-wire Probes and Anemometer 22

2.3.3.1 Probe Design 22

$\begin{array}{ll}\text { 2.3.3.2 Anemometer } & 27\end{array}$

2.3.4 Low Frequency Data Acquisition- Labview 27

2.3.5 High Frequency Data Acquisition - LeCroy 28 
2.4 TURBULENCE GRID 28

2.5 MEASUREMENT UNCERTAINTY 31

Chapter 3 Data Analysis and Results 32

$\begin{array}{lll}3.1 & \text { TURBUlenCE } & 32\end{array}$

3.1.1 Definition of Turbulence 32

3.1.2 Characterizing Turbulence 33

3.1.2.1 Time-Resolved Velocity Signal Decomposition 33

3.1.2.2 Turbulence Intensity and Velocity Spectrum 34

3.1.2.3 Length Scale and Autocorrelation 36

3.1.2.4 Energy Spectra Relations and Isotropic Turbulence 38

3.1.2.5 Coherence 40

3.2 Steady Surface Heat Transfer 41

3.2.1 High Speed Flow Heat Transfer (from Nix, 1996) 41

3.2.2 Steady Heat Transfer Coefficient Methodology 42

3.2.3 Steady Heat Transfer Results 46

3.2.3.1 Low Turbulence Heat Transfer Coefficients 46

3.2.3.2 High Turbulence Heat Transfer Coefficients 47

3.3 Time-Resolved Velocity AND HeAt TRANSFER 50

3.3.1 Time Domain Analysis $\quad 51$

3.3.1.1 Direct Time Signal Comparison 51

3.3.1.2 Time Correlation and Scales 55

3.3.2 Frequency Domain Analysis 58

3.3.2.1 Velocity and Heat Flux Spectra 58

3.3.2.2 Coherence 62

3.4 Analytical Model 64

3.4.1 Analytical Model Development 64

3.4.2 Single-scale Model Results 68

3.4.2.1 Application to Transonic Facility Data 68

3.4.2.2 Application to Other Data Sets 70

3.4.2.3 Stagnation Point Data - Diller and Van Fossen, 199671 
3.4.2.4 Low speed turbine vane data - Radomsky and Thole, 1998

3.4.2.5 Low speed turbine vane pressure surface data - Ames, 1994

3.4.2.6 Turbulent flat plate data - Moss and Oldfield, $1992 \quad 79$

3.4.2.7 Transonic turbine blade data - Holmberg, 1996

3.4.2.8 Film-cooled Transonic turbine blade data - Smith, et al., 2000

3.4.3 Comparison with Other Correlations

3.4.3.1 Dullenkopf and Mayle (1995) Effective Turbulence Correlation

3.4.3.2 Maciejewski and Moffat (1992) St' Correlation

3.4.3.3 Van Fossen, Simoneau and Ching (1995) Frossling Number Correlation

3.4.4 Multiple-scale Model

3.4.4.1 Development of Multiple-scale Model 90

3.4.4.2 Application of Multiple-scale Model 92

3.4.4.3 Combination of Multiple-scale Model Data 97

3.4.4.4 Application of Multiple-scale Model to Other Data 108

Chapter 4 CONClusions

4.1 SUMMARY

4.2 Application to Turbine Design

4.3 OUTLOOK AND FUtURE WORK

\section{APPENDICES}

Appendix A - Turbulence Generation Paper and Presentation 


\section{LIST OF TABLES}

1.1 Review of Turbulence Generation Techniques 9

2.1 Non-dimensional conditions and cascade conditions and geometry 15

$\begin{array}{lll}2.2 & \text { Blade Measurement Locations } & 18\end{array}$

2.3 Summary of total uncertainty $(\Delta) \quad 31$

3.1 Summary of heat transfer coefficients with low turbulence 47

3.2 Summary of heat transfer coefficients with high turbulence 48

3.3 Turbulence and heat flux intensity 53

3.4 Time scales and integral length scales 55

3.5 Results of single-scale model applied to transonic tunnel data 69

$\begin{array}{lll}3.6 & \text { Diller (1996) heat transfer data } & 71\end{array}$

3.7 Results of single-scale model applied to Diller (1996) data 72

3.8 Model results compared to data from Moss and Oldfield (1992) 80

3.9 Model results compared to data from Holmberg (1996) 82

3.10 Analytical model applied to film-cooled data from Smith, et al. (2000) 84

3.11 Multiple-scale model results by various methods for location PS1 104

3.12 Multiple-scale model results by various methods for locations PS2 and SS1 107

3.13 Multiple-scale model results by various methods for data from Diller and Van Fossen (1996) 108 


\section{LIST OF FIGURES}

2.1 Virginia Tech transonic blowdown wind tunnel facility 13

$\begin{array}{lll}2.2 & \text { Typical tunnel inlet total pressure plot } & 14\end{array}$

2.3 Cascade test section illustration showing instrumented blade location $\quad 15$

2.4 Predicted Mach number distribution for the turbine blades 16

2.5 Turbine blade showing measurement locations and instrumentation details 17

2.6a Vatell HFM-7 Heat Flux Microsensor 19

2.6b Unmounted Kulite pressure transducer 22

2.7a Drawings of custom hot-wire probes used in experiments (Edited from Holmberg, 1996) 23

$2.7 \mathrm{~b}$ Pictures of hot-wire probes used in experiments 24

2.8 Picture of PHW probe mounted above heat flux gage on blade surface 26

2.9 Turbulence generating grid used in the facility 29

3.1 Illustration of velocity time signal and decomposition 33

3.2 Example of velocity power spectral density (PSD) 36

3.3 Typical autocorrelation curves $\left(\mathrm{R}_{\text {uи }}\right) \quad 38$

3.4 Normalized PSD and Von Kármán relation 40

3.5a Time history of relevant temperatures (SS1) 44

$3.5 \mathrm{~b}$ Heat transfer data analysis (SS1) 45

3.6 Time history of heat flux and heat transfer coefficient (SS1) 46

3.7 Heat transfer coefficient data compared with KEP code $\begin{array}{ll}\text { predictions and previous data } & 50\end{array}$

3.8 Illustration of simultaneous velocity and heat flux measurements 51

3.9a Time-resolved velocity and heat flux signals - Location SS1 54

3.9b Time-resolved velocity and heat flux signals - Location PS1 54

3.9c Time-resolved velocity and heat flux signals - Location PS2 55 
3.10a Autocorrelation of velocity and heat flux signals - Location SS1 56

3.10b Autocorrelation of velocity and heat flux signals - Location PS1 57

3.10c Autocorrelation of velocity and heat flux signals - Location PS2 57

3.11a Velocity and heat flux spectra - Location SS1 58

3.11b Velocity and heat flux spectra - Location PS1 59

3.11c Velocity and heat flux spectra - Location PS2 59

3.12 Comparison of velocity spectra at all measurement locations 60

3.13 Comparison of heat flux spectra at all measurement locations 60

3.14 Normalized velocity spectra and Von Kármán relation 61

3.15 Coherence between velocity and heat flux signals 63

3.16 Illustration of turbulent heat transfer model 65

3.17a Comparison of model predicted and measured Stanton numbers (St) from Radomsky and Thole (1998) - 10\% Turbulence Case 74

3.17b Comparison of model predicted and measured Stanton numbers (St) from Radomsky and Thole (1998) - 20\% Turbulence Case 74

3.18 Comparison of model predicted and measured $\mathrm{St} / \mathrm{St}_{\mathrm{o}}$ from Radomsky and Thole (1998)

3.19 Comparison of model predicted and measured Stanton numbers (St) from Ames (1994)

3.20 Comparison of model predicted and measured $\mathrm{St} / \mathrm{St}_{\mathrm{o}}$ from Ames (1994) 78

3.21 Comparison of model predicted and measured $\mathrm{St} / \mathrm{St}_{0}$ from Ames (1994) for Comb 2 with error estimates

3.22 Comparison with Dullenkopf and Mayle (1995) effective turbulence Correlation $\quad 86$

3.23 Comparison with Maciejewski and Moffat St' correlation 87

3.24 Comparison with Van Fossen, et al. (1995) Frossling number $\begin{array}{ll}\text { Correlation } & 89\end{array}$

3.25 Model predicted heat flux increase as a function of frequency 92

3.26 Comparison of model predicted heat flux increases with measured $\begin{array}{ll}\text { Fluctuations } & 93\end{array}$

3.27 Coherence between velocity and heat flux signals 95

3.28a Predicted model heat flux with coherence applied and measured heat flux fluctuations 
3.28b Predicted model heat flux with coherence applied and measured heat flux fluctuations (Linear scale)

3.29 Illustration of increase in heat transfer by turbulent fluctuations in heat flux

98

3.30 Illustration of frequency band of multiple-scale heat flux model

106 


\section{NOMENCLATURE}

\section{Symbols}

a

B

C

$\mathrm{C}_{\mathrm{p}}$

c

$\mathrm{c}_{\mathrm{a}}$

$\mathrm{c}_{\mathrm{ps}}$

$\mathrm{c}_{\mathrm{ss}}$

D

$\mathrm{D}_{\mathrm{s}}$

d

E(f)

$\mathrm{f}$

$\mathrm{f}_{\mathrm{s}}$

$\mathrm{f}_{\Lambda}$

Fr

$\mathrm{G}_{\mathrm{HFS}}$

$\mathrm{G}_{\mathrm{RTS}}$

$\mathrm{G}_{\mathrm{p}}$

$\mathrm{h}$

$\overline{\mathrm{h}}$

$h_{\text {rms }}^{\prime}$

$\mathrm{h}_{\mathrm{o}}$
Freestream strain rate

Bias uncertainty

Hot-wire calibration constant

Specific heat

Blade chord length

Aerodynamic chord

Chord length of the pressure surface

Chord length of the suction surface

Characteristic diameter (in Strouhal number)

Heat flux sensor footprint diameter

Cylinder or blade leading edge diameter

Power spectral density (energy spectrum) (PSD)

Frequency

Vortex shedding frequency

Frequency corresponding to integral length scale in multiple-scale model

Frossling number

Amplifier gain of heat flux sensor (HFS)

Amplifier gain of resistance temperature sensor (RTS)

Amplifier gain of pressure transducer

Convection heat transfer coefficient

Mean heat transfer coefficient

Root mean square of fluctuating heat transfer coefficient

Low turbulence heat transfer coefficient 


\begin{tabular}{|c|c|}
\hline$h_{o, c}$ & Film-cooled low turbulence heat transfer coefficient \\
\hline $\mathrm{h}_{\mathrm{t}}, \mathrm{h}_{\text {turb }}$ & High turbulence heat transfer coefficient \\
\hline$h_{t, c}$ & High turbulence heat transfer coefficient with film cooling \\
\hline$h$ & Enthalpy \\
\hline$h_{t}$ & Total enthalpy \\
\hline I & Momentum flux ratio \\
\hline $\mathrm{I}_{\mathrm{RTS}}$ & Current applied to resistance temperature sensor (RTS) \\
\hline $\mathrm{k}$ & Thermal conductivity \\
\hline $\mathrm{L}_{\mathrm{a}}$ & Length scale including strain rate \\
\hline $\mathrm{Lu}$ & Streamwise integral length scale \\
\hline $\mathrm{L}_{\mathrm{u}} \mathrm{e}^{\mathrm{a}}$ & Dissipation length scale \\
\hline M & Mach number \\
\hline$N$ & Number of samples \\
\hline $\mathrm{Nu}$ & Nusselt number \\
\hline $\mathrm{Nu}^{\prime}$ & Modified Nusselt number for hot-wire calibration \\
\hline $\mathrm{Nu}_{\mathrm{a}}$ & Nusselt number based on strain rate \\
\hline $\mathrm{Nu}_{\mathrm{o}}$ & Low turbulence Nusselt number \\
\hline $\mathrm{P}$ & Precision uncertainty \\
\hline $\mathrm{P}_{\mathrm{s}}$ & Static temperature \\
\hline $\mathrm{P}_{\mathrm{t}}$ & Total pressure \\
\hline $\mathrm{p}$ & Blade pitch \\
\hline $\operatorname{Pr}$ & Prandtl number \\
\hline PS1 & Measurement location on the pressure surface \\
\hline PS2 & Measurement location on the pressure surface \\
\hline q, q" & Heat flux \\
\hline$q^{\prime}$ & Fluctuating component of heat flux \\
\hline $\mathrm{q}_{\text {'rms }}^{\prime}$ & Root mean square of fluctuating heat flux \\
\hline Qf & FFT of q' \\
\hline $\mathrm{Qf}_{\mathrm{mag}}$ & Magnitude of Qf \\
\hline $\mathrm{R}$ & Resistance (of RTS sensor) \\
\hline $\mathrm{R}_{\mathrm{xx}}$ & Autocorrelation of variable $\mathrm{X}$ \\
\hline
\end{tabular}




\begin{tabular}{|c|c|}
\hline $\mathrm{R}_{\mathrm{o}}$ & RTS sensor resistance at room temperature \\
\hline $\mathrm{r}$ & Recovery factor \\
\hline $\operatorname{Re}$ & Reynolds number \\
\hline $\operatorname{Re}_{\delta}$ & Reynolds number based on boundary layer thickness $(\delta)$ \\
\hline $\operatorname{Re}_{\theta}$ & Reynolds number based on momentum thickness $(\theta)$ \\
\hline $\mathrm{Re}_{\mathrm{w}}$ & Reynolds number of hot-wire \\
\hline S & Strouhal number \\
\hline$S_{p}$ & Sensitivity of pressure transducer \\
\hline$S_{\mathrm{xx}}$ & Auto spectrum of variable $X$ \\
\hline $\mathrm{S}_{\mathrm{xy}}$ & Cross spectrum of variables $X$ and $Y$ \\
\hline sp & Span \\
\hline SS1 & Measurement location on the suction surface \\
\hline St & Stanton number \\
\hline $\mathrm{St}^{\prime}$ & Modified Stanton number based on $\mathrm{u}_{\mathrm{rms}}^{\prime}$ \\
\hline $\mathrm{St}_{\mathrm{o}}$ & Low turbulence Stanton number \\
\hline $\mathrm{T}$ & Temperature \\
\hline$T$ & Time period of sample \\
\hline $\mathrm{T}_{\mathrm{d}}$ & Difference between recovery temperature and total temperature \\
\hline $\mathrm{T}_{\mathrm{e}}$ & Eddy temperature \\
\hline $\mathrm{T}_{\mathrm{q}}$ & Time scale of heat flux \\
\hline $\mathrm{T}_{\mathrm{r}}$ & Recovery temperature \\
\hline $\mathrm{T}_{\mathrm{s}}$ & Static temperature \\
\hline $\mathrm{T}_{\mathrm{t}}$ & Total temperature \\
\hline $\mathrm{T}_{\mathrm{u}}$ & Time scale of velocity \\
\hline $\mathrm{T}_{\mathrm{w}}$ & Wall temperature (blade surface) \\
\hline $\mathrm{T}_{\infty}$ & Freestream static temperature \\
\hline $\mathrm{t}$ & Time (residence time in model) \\
\hline TLR & Turbulence parameter using integral parameters (Moffat, et al.) \\
\hline $\mathrm{Tu}$ & Turbulence intensity \\
\hline $\mathrm{Tu}_{\mathrm{a}}$ & Turbulence intensity including strain rate \\
\hline $\mathrm{T}_{\mathrm{hw}}$ & Hotwire temperature \\
\hline
\end{tabular}




$\begin{array}{ll}\mathrm{Tu}_{\mathrm{q}} & \text { Heat flux intensity } \\ \mathrm{Tu}_{\lambda} & \text { D\&M turbulence parameter } \\ \mathrm{t}^{\prime} & \text { Temperature fluctuations } \\ \mathrm{U}_{,} \mathrm{U}_{\infty} & \text { Freestream velocity } \\ \bar{U} & \text { Mean streamwise velocity } \\ \mathrm{U}_{\mathrm{e}} & \text { Boundary layer edge velocity } \\ \widetilde{u} & \text { Instantaneous streamwise velocity } \\ \mathrm{u}_{\mathrm{j}} & \text { Velocity of active grid jet } \\ \mathrm{u}_{\infty} & \text { Freestream velocity } \\ \mathrm{u}^{\prime} & \text { Streamwise velocity fluctuations } \\ \mathrm{u}_{\mathrm{eff}}^{\prime} & \text { Dullenkopf \& Mayle effective turbulence level } \\ \mathrm{u}_{\mathrm{rms}}^{\prime} & \text { Root mean square of the fluctuating component of streamwise velocity } \\ \mathrm{Uf}^{\prime} & \text { FFT of u' } \\ \mathrm{Uf}_{\mathrm{mag}} & \text { Magnitude of Uf } \\ \mathrm{V} & \text { Hot-wire voltage } \\ \mathrm{V}_{\mathrm{HFS}} & \text { Voltage output of heat flux sensor (HFS) } \\ \mathrm{V}_{\mathrm{p}} & \text { Voltage output of pressure transducer } \\ \mathrm{V}_{\mathrm{RTS}} & \text { Voltage output of resistance temperature sensor (RTS) } \\ \mathrm{v}^{\prime} & \text { Cross-stream velocity fluctuations } \\ \mathrm{w}^{\prime} & \text { Lateral velocity fluctuations } \\ \mathrm{x} & \text { Distance along surface (blade, flat plate, etc) } \\ \mathrm{X}(\mathrm{f}) & \text { FFT of variable X }\end{array}$

\section{Greek Symbols}

$\alpha \quad$ Thermal diffusivity

$\gamma \quad$ Specific heat ratio $\left(\mathrm{C}_{\mathrm{p}} / \mathrm{C}_{\mathrm{v}}\right)$

$\gamma_{\text {uq }}^{2} \quad$ Coherence between velocity and heat flux

$\gamma_{x y}^{2} \quad$ Coherence function of variables $X$ and $Y$

$\Delta \quad$ Total uncertainty

$\Delta \mathrm{f} \quad$ Frequency resolution (bandwidth)

$\Delta \mathrm{f}_{\mathrm{b}} \quad$ Frequency band centered on integral scale 
$\Delta \phi \quad$ Dimensionless frequency band centered on integral scale

$\Delta \mathrm{h}_{\mathrm{t}} \quad$ Increase in heat transfer coefficient due to turbulence

$\Delta \mathrm{q}_{\mathrm{t} \text {, total }} \quad$ Total of heat flux increases in frequency domain

$\Delta \mathrm{T} \quad$ Temperature difference

$\Delta \mathrm{t} \quad$ Time per sample point

$\delta \quad$ Boundary layer thickness

$\theta \quad$ Momentum thickness

$\kappa \quad$ Wave number

$\kappa_{\mathrm{e}} \quad$ Most energetic wave number

$\Lambda_{\mathrm{x}} \quad$ Streamwise integral length scale

$v \quad$ Kinematic viscosity

$\rho \quad$ Density

$\rho_{j} \quad$ Density of active grid jet

$\rho_{\infty} \quad$ Density of freestream flow

$T \quad$ Integral timescale

$\tau \quad$ Time lag in autocorrelation

$\phi_{\lambda} \quad$ Dimensionless frequency centered on integral length scale 


\section{CHAPTER 1}

\section{INTRODUCTION}

\subsection{Turbine Blade Heat Transfer - Problem Statement}

Gas turbine engine designers continually strive to improve engine performance by increasing overall engine efficiency and thrust-to-weight ratio. Improvements in overall engine efficiency are driven in large part by increasing the combustor exit temperature and turbine inlet temperature. Increasing thrust-to-weight ratio necessitates the use of lighter materials, which may operate near their thermal limits.

The implementation of blade cooling techniques in conjunction with advances in blade materials has increased the envelope of turbine inlet temperatures. With the blades operating near their thermal limits, it is critical to the engine designer to have accurate predictions of hot mainstream gas to blade surface heat transfer rates. The heat transfer to the turbine blades is composed of steady heat transfer based on mean-flow conditions, as well as unsteady heat transfer due to fluctuations in the mean flow. The unsteadiness of the flowfield is a product of wake passing and shock waves from blade row interactions and freestream turbulence generated in the combustor. The effects of these unsteady features of the gas turbine flowfield on blade heat transfer need to be quantified to provide more accurate predictions of blade surface heat transfer and facilitate the development of more effective cooling schemes.

Analysis of the effects of flow unsteadiness on heat transfer is often performed by decoupling the effects of the unsteady flow phenomena and studying each independently. Studies range from simple flat plate and cylinder stagnation point analyses to cascade testing and full rotating rigs. Rotating rigs simulate turbine-operating conditions more accurately, however they often make it difficult to separate the effects of various unsteady flow phenomena. Cascade testing provides the ability to study heat transfer matching many engine conditions while decoupling the effects of various unsteady flow phenomena. 
The focus of the current research work is to investigate the effects of freestream turbulence on turbine blade heat transfer in a transonic cascade with high turning turbine blades. The steady flowfield matches engine non-dimensional conditions (Mach number and Reynolds number) and the generated turbulence field, intensity and length scale, is consistent with recent reports from modern gas turbine engine combustors (Van Fossen and Bunker, 2002) and reports of turbulence decay through the first stage vane row (Radomsky and Thole, 1998, 2000). The turbulence field simulates the core flow turbulence in the turbine, decoupling this turbulence from the turbulence in the wake. The use of high frequency instrumentation allows for the analysis of both time-averaged and time-resolved heat transfer.

Following is a review of the literature of investigations of the effects of turbulence on heat transfer. The review attempts to cover the broad spectrum of studies which have been performed on the topic and also reviews turbulence generation techniques and time-resolved measurements of heat flux.

\subsection{LiteratURE REVIEW}

\subsubsection{Turbulent Heat Transfer Studies and Correlations}

Many studies have been performed in the last 25 years focusing on quantifying the effects of freestream turbulence on heat transfer. Much of the previous work has been experimental analyses on simplified geometries, namely the flat plate and cylinder in cross-flow. Flat plate experiments present the most basic geometry for experiments, with the absence of curvature and pressure gradient effects. Cylinder experiments are of particular importance as the stagnation point is typically the region of highest heat transfer on turbine vanes and blades except for regions of transitional and turbulent flow. Cylinder experiments also provide data on the effects of curvature and pressure gradient. In more recent years, studies have been performed in cascades of turbine blades and vanes. These studies present data sets that are generally more applicable to real gas turbine engines. The following review of previous work will be presented by grouping experiments together by classification of the experiments, rather than presenting a chronological succession of studies. 


\subsubsection{Flat Plate Experiments}

Several research groups have performed flat plate studies of turbulent heat transfer. Bradshaw (1974) and Hancock and Bradshaw (1983) studied the effects of freestream turbulence on turbulent flat plate boundary layers. Data were collected with and without turbulence and comparisons between the data sets were made at similar Reynolds numbers based on boundary layer thickness $\left(\operatorname{Re}_{\theta}\right.$ and $\left.\operatorname{Re}_{\delta}\right)$. The level of freestream turbulence in these experiments was rather low, with reported intensity at $7 \%$ and below. A correlation was developed which correlates increases in skin friction with turbulence intensity, dissipation length scale $\left(\mathrm{L}_{u}{ }^{e}\right)$ and boundary layer thickness $(\delta)$. Reynolds number was reported to have no effect on how freestream turbulence affected skin friction enhancement.

Blair (1983) correlated skin friction and heat transfer increases due to freestream turbulence on turbulent flat plates and demonstrated that increases in freestream turbulence induce upstream boundary layer transition. His work showed that there was a low Reynolds number effect that attenuated skin friction enhancement and modified the correlation of Hancock and Bradshaw to include low Reynolds number effects. Measured heat transfer enhancement was shown to increase at a higher rate than skin friction with increasing turbulence intensity. The Reynolds analogy factor was demonstrated to increase linearly with increasing freestream turbulence levels. The work of Blair was later corrected based on additional low Reynolds number data by Castro (1984). It was shown that the low Reynolds number effect was less significant at higher turbulence intensities. Moss and Oldfield (1992) combined the data sets of Blair and Castro with their own data, which included higher turbulence intensity (up to 16\%) and modified the correlation of Blair. Work by MacMullin, et al. (1989), Maciejewski and Moffat (1989, 1992), Ames and Moffat (1990) and Sahm and Moffat (1992) also extended the range of the correlation developed by Blair and proposed a new parameter (TLR) which uses integral quantities rather than boundary layer thickness, since defining the edge of the boundary layer at high levels of freestream turbulence can be difficult.

Maciejewski and Moffat (1989) presented flat plate turbulent boundary layer data with turbulence intensities as high as $60 \%$ using a free jet. They proposed a simpler method of analyzing high turbulence heat transfer data. They observed a linear 
relationship between measured heat transfer coefficient (h) and turbulent fluctuations in the freestream $\left(u^{\prime}\right)$. Their correlation was independent of geometry or length scale, and was simply a function of near wall turbulence level. Although the range of turbulence intensity is much higher than what is believed to be representative of turbine conditions, these results are of interest. Later work by Maciejewski and Moffat (1992), Maciejewski and Anderson (1996) and Denninger and Anderson (1999) revised the correlation of Maciejewski and Moffat (1989) using the value of $\mathrm{u}_{r m s, \max }^{\prime}$ in the inner wall region as an input parameter to improve the correlation and extend it's applicability to lower levels of freestream turbulence.

Thole and Bogard (1995) performed a comprehensive analysis of all of these works and reported that the correlation using integral quantities is more successful in scaling heat transfer enhancement, while Blair's correlation is more appropriate for skin friction enhancement predictions. They also concluded that the correlation of Maciejewski and Moffat (1992) is good for cases of very high freestream turbulence $(\mathrm{Tu}>20 \%)$. Relative to the differences in measured heat transfer and skin friction enhancements in the presence of high freestream turbulence, they conclude that the difference is due to the fact that at higher levels of turbulence, large scale eddies penetrate into the boundary layer. These large scale eddies are effective at removing heat, but are non-stress producing, so at higher turbulence levels, they continue to enhance heat transfer, but their affect on shear stress is attenuated.

Barrett and Hollingsworth (2003) studied the effects of very small scale (ratio of length scale to boundary layer momentum thickness of 4 to 32$)$, moderate intensity (0.1 $8 \% \mathrm{Tu}$ ) turbulence on skin friction. They developed a new boundary layer model to establish a skin friction correlation that does not rely on knowledge of the momentum thickness Reynolds number.

\subsubsection{Cylinder Experiments}

Many of the original works studying the effects of freestream turbulence on heat transfer were cylinder and leading edge studies. Since the flow in the stagnation region can be simulated by studying a cylinder in cross-flow, these studies represent a simple but effective experimental analysis which has a valid application to turbine vane and blade studies. As mentioned, the heat transfer in the leading edge region of turbine 
blades and vanes is typically the region of highest heat transfer with the exception of regions of transitional and turbulent flow.

Smith and Keuthe (1966), Kestin and Wood (1971) and Lowery and Vachon (1975) developed and modified correlations that correlated Nu/Re ${ }^{1 / 2}$ (Frossling number) against $\mathrm{TuRe}^{1 / 2}$. The correlations were only a function of the turbulence intensity (Tu) and cylinder Reynolds number (Re), length scale was not included in these studies as it was in later work on cylinders and in most of the work on flat plates. Mehendale, et al (1991) later modified the correlation for a larger range of turbulence levels up to $15 \%$.

Van Fossen and Simoneau (1985) performed a streamwise traverse of a hot-wire along the stagnation streamline for a cylinder in cross-flow. From measurement of velocity fluctuations approaching the leading edge of the cylinder it was determined that vorticity increases as it approaches the stagnation region. This has been attributed to vortex stretching around the leading edge.

Van Fossen, et. al (1995) studied the influence of turbulence on heat transfer on cylinders and cylinders with wedge shaped afterbodies. In these studies, it was determined that augmentation of stagnation region heat transfer increases as integral length scale decreases. A correlation was developed which modified those of previous cylinder studies to include the integral length scale in the form of the ratio of the length scale to cylinder leading edge diameter $\left(\Lambda_{\mathrm{x}} / \mathrm{d}\right)$. This type of correlation may have direct application to turbine blade leading edge heat transfer predictions, using an effective diameter based on potential flow approaching the leading edge. Since combustor turbulence, as will be discussed later, has been shown to be of fairly large scale, the lower bound of the correlation presented by Van Fossen may not be of particular interest in direct application to turbine design.

Dullenkopf and Mayle (1995) presented a new correlation that introduced the idea of an "effective" turbulence level. The correlation was developed based on stability theory for a laminar boundary layer. Fluctuations at high frequencies (small scale) are viscously damped, while disturbances at low frequency (large scale) are seen as quasisteady similar to bulk flow pulsations, which in the mean do not affect heat transfer. Only a small frequency band around a dominant frequency is effective at increasing heat transfer. A new turbulence parameter $\left(\mathrm{Tu}_{\lambda}\right)$ was introduced which accounts for effective 
turbulence level and length scale. They introduced a Stanton number that was linearly proportional to the effective turbulence level ( $u_{\text {eff }}^{\prime}$, similar to the results proposed by Maciejewski and Moffat (1992).

Van Fossen and Bunker (2002) measured stagnation region augmentation downstream of a section of a dual-annular combustor. The turbulence intensity and length scale downstream of the combustor section were mapped out using a two component hot-wire. The turbulence field was shown to have a maximum intensity of approximately $32 \%$ with an integral length scale of $1.29 \mathrm{~cm}$. Heat transfer augmentation was predicted well by correlations. The reported turbulence levels exiting the combustor were somewhat higher than measured by other researchers (Goldstein, et al., 1983, Kuotmos and McGuirk, 1989 and Ames 1994, 1995 and 1997) where levels of 20-25\% were measured.

\subsubsection{Turbine Vane and Blade Experiments}

Several research groups have performed experiments on turbine vane geometries in low speed cascade facilities. Cascade experiments present a more realistic simulation of the effects of turbulence on turbine heat transfer and results are generally more applicable to turbine conditions than flat plate data.

Ames $(1995,1997)$ performed measurements of the effects of large-scale freestream turbulence in a low speed turbine vane cascade. In these studies, three different turbulence fields were generated with varying intensity and scale. Freestream turbulence was shown to significantly augment heat transfer in the laminar regions of the blade (stagnation point, pressure surface and favorable pressure gradient region of the suction surface). Heat transfer augmentation was reported to be highest in the stagnation region and on the pressure surface. Stagnation region increases agreed well with cylinder stagnation point correlations. The average augmentation of heat transfer on the pressure surface was found to scale well with the inlet turbulence intensity and the length scale to the $-1 / 3$ power $\left(\mathrm{TuLu}^{-1 / 3}\right)$. Comparisons of Stanton number increases on both the pressure and suction surfaces of the vane demonstrated that turbulence has a much greater effect on the pressure surface of the vane as compared to the suction surface. Increased turbulence slightly shifted suction surface transition towards the leading edge. 
In the turbulent region of the suction surface, increased turbulence had a smaller effect on heat transfer.

Radomsky and Thole $(1998,2000,2002)$ documented surface heat transfer and the turbulent flowfield around a scaled up stator vane. Several different turbulence levels as high as $25 \%$ were generated. Observations of heat transfer augmentation were consistent with results reported by Ames (1995), with heat transfer augmentation being greatest on the pressure surface and in the stagnation region and suction surface transition shifting toward the leading edge at elevated freestream turbulence levels. In Radomsky and Thole (1998), detailed measurements of unsteady velocity upstream of the vane passage and along a streamline in the center of the vane passage were used to document the levels of turbulence kinetic energy and all three unsteady components of velocity through the vane passage. The turbulent kinetic energy was shown to increase through most of the blade passage. The streamwise velocity fluctuations $\left(u^{\prime}\right)$ were shown to decrease through the blade passage, while cross-stream $\left(v^{\prime}\right)$ and lateral $\left(w^{\prime}\right)$ fluctuations increased. Detailed measurements using a 3 component LDV system were reported in Radomsky and Thole (2000) and showed that turbulence remains quite high throughout the passage. Length scale was shown to decrease through the blade passage, although not considerably. The effect of turbulent length scale was reported to have the largest effect on the pressure surface of the vane, with lower Stanton numbers at the larger scales.

Mehendale, et al. (1994) and Wang, et al. (1998) measured the effects of high intensity, large scale turbulence on turbine blade heat and mass transfer in a low speed cascade at varying turbulence levels. In general, results of their tests agreed with those from turbine vane testing in terms of locations of highest heat and mass transfer and early boundary layer transition on the suction surface. Leading edge results agreed well with published correlations from Kestin and Wood (1971) and Dullenkopf and Mayle (1995).

Holmberg (1996) studied the effects of small-scale, relatively low intensity turbulence on heat transfer in high-turning turbine blades in transonic flow and measured time-resolved heat flux and velocity. Coherence between freestream velocity and surface heat flux was found to be useful in determining the scale and frequency range of turbulent structures interacting with surface heat flux. This study is similar to the current work except with lower intensity, smaller scale turbulence. 


\subsubsection{Turbulence Generation Methods}

Experimental measurements of turbulent heat transfer cannot, of course, be performed without the means of generating the desired turbulent flowfield. One consequence of the many turbulence studies discussed in the previous section is a vast array of turbulence generation techniques. A fairly comprehensive discussion is presented in Nix, et al. (2002) in Appendix A. The paper breaks down the generation techniques into two categories, mock combustor/jets in crossflow and grid or bar generators. A summary of the grid generation techniques is presented in Table 1.1.

From the summary, it can be seen that active blowing (either through jets in cross flow or through active grids or bar arrays) and passive bar arrays with large flow blockage are effective at producing high levels of turbulence. The current work attempted to use active blowing in the transonic facility, as discussed in detail later in section 2.4, but the momentum flux ratio $\left(I=\rho_{j} u_{j}{ }^{2} / \rho_{\infty} u_{\infty}{ }^{2}\right)$ required was not achievable. The grid used in this work was a large blockage grid based on the results reported by Polanka and Bogard (1999).

\subsubsection{Time-Resolved Heat Transfer}

The analyses presented later in this work rely on the measurement of timeresolved heat transfer and velocity. Limited time-resolved heat flux experiments exist in the open literature. Heat flux microsensors have been used to measure time-resolved heat flux at Virginia Tech by Johnson (1995), Holmberg (1996), Nix, et al. (1997a, b), and Popp, et al. (2000). Time-resolved measurements were performed to measure unsteady fluctuations and unsteady shock passing events.

Thin film heat flux gages have been used to measure unsteady heat transfer in rotating rigs at CALSPAN by Dunn (1986, 1989), at MIT by Guenette (1989) and at Oxford by Ashworth (1989), Garside et al. (1994), by Moss and Oldfield $(1992,1996)$ and by Anthony, et al (1999) at Oxford and Syracuse.

Of the time-resolved heat flux data sets published in the open literature, only three included simultaneous measurements of velocity. Moss and Oldfield $(1992,1996)$ and the previous work by Holmberg (1996) and Holmberg and Pestian (1996) are the exceptions. 
Table 1.1: Review of Turbulence Generation Techniques

\begin{tabular}{|c|c|c|c|c|c|}
\hline Investigator & $\begin{array}{c}\text { Turbulence } \\
\text { Level }\end{array}$ & $\begin{array}{l}\text { Length } \\
\mathrm{cm}\end{array}$ & $\begin{array}{l}\text { Scale } \\
\left(\Lambda_{x} / c\right)\end{array}$ & Method & $\begin{array}{c}\text { Flow } \\
\text { Condition }\end{array}$ \\
\hline $\begin{array}{l}\text { Mehendale and Han } \\
\text { (1992) }\end{array}$ & $13 \%$ & 3 & - & Blown Grid & Low speed \\
\hline $\begin{array}{l}\text { Maciejewski and } \\
\text { Moffat (1992) }\end{array}$ & $20-60 \%$ & - & - & Turbulent free jet & - \\
\hline $\begin{array}{l}\text { Sahm and Moffat } \\
\text { (1992) }\end{array}$ & $\begin{array}{c}4-9 \% \\
13-21 \%\end{array}$ & - & - & $\begin{array}{l}\text { Grid, blown grid and } \\
\text { jets in crossflow }\end{array}$ & - \\
\hline Thole, et al. (1994) & $20 \%$ & $2-3$ & - & Jets in crossflow & Low speed \\
\hline Holmberg (1996) & $5 \%$ & 0.5 & $<0.1$ & Grid & Transonic \\
\hline $\begin{array}{l}\text { Schauer and } \\
\text { Pestian (1996) }\end{array}$ & $17 \%$ & 8 & - & Jets in crossflow & Low speed \\
\hline Ames (1997) & $13 \%$ & 1.6 & 0.11 & Mock combustor & Low speed \\
\hline Boyle, et al. (1998) & $10 \%$ & 1.5 & - & $\begin{array}{l}\text { Blown and unblown } \\
\text { grid }\end{array}$ & Low speed \\
\hline Wang, et al. (1998) & $18 \%$ & 8 & 0.43 & Mock combustor & Low speed \\
\hline $\begin{array}{l}\text { Radomsky and } \\
\text { Thole (2000) }\end{array}$ & $20 \%$ & $5-6$ & - & Blown bar array & Low speed \\
\hline $\begin{array}{l}\text { Polanka and } \\
\text { Bogard (1999) }\end{array}$ & $20 \%$ & 5 & - & $\begin{array}{l}\text { Passive bar array } \\
\text { (large blockage) }\end{array}$ & Low speed \\
\hline Giel, et al. (2000) & $10 \%$ & 2.2 & 0.17 & Active grid & Transonic \\
\hline $\begin{array}{l}\text { Barringer, et al. } \\
(2002)\end{array}$ & $18 \%$ & $6-7$ & 0.11 & $\begin{array}{l}\text { Combustor } \\
\text { simulator with large } \\
\text { jets }\end{array}$ & Low speed \\
\hline
\end{tabular}

Moss and Oldfield $(1992,1996)$ traversed a hot-wire probe above a flat plate with thin film gages on the surface. They performed time-domain analyses of the timeresolved signals, comparing raw signals and cross correlations between the velocity and heat flux signal and between heat flux signals of different gages. The raw heat flux time signal showed large and small fluctuations. The large fluctuations were attributed to freestream turbulent eddies acting on the surface, while the small fluctuations were due to turbulence within the boundary layer. Through correlations of velocity and heat flux and spatial correlations between heat flux gages they concluded that the turbulent eddy structure of the boundary layer is dominated by the freestream turbulence and eddies within the boundary layer convected at the freestream velocity, $\mathrm{U}$, rather than at $\sim 0.8 \mathrm{U}$ characteristic of boundary layers. 
Holmberg (1996), discussed earlier, performed measurements of unsteady surface heat flux with a hot-wire collocated with the heat flux gage. Holmberg and Pestian (1996) measured $u^{\prime}$ and $v^{\prime}$ velocity components and fluctuating surface temperature ( $\left(t^{\prime}\right)$ and heat flux (q') in a low speed wall-jet facility. Surface heat flux was shown to be dominated by the u' fluctuating velocity field primarily by large freestream eddies which reach deep into the boundary layer. These results are consistent with the observations of Moss and Oldfield $(1992,1996)$.

\subsection{OVERVIEW OF CURRENT WORK}

The current experimental work at Virginia Tech studying the effects of freestream turbulence extends work performed by Holmberg (1996). The blade geometry is the same as that studied by Holmberg, but has been scaled up three times larger than the previous work. The advantage of the increase in blade size is that it decreases the relative size of the heat flux sensor footprint compared to the size of the blade surface. This decreases spatial averaging due to better spatial resolution compared to previous experiments. Another advantage to the increase in scale is that the pitch to chord ratio $(\mathrm{p} / \mathrm{c})$ has not changed, so the ratio of the diameter of the hot-wire probes used to make passage measurements to the passage width is much smaller. This minimizes any flow disturbance which may be caused by the hot-wire probe support. Probe disturbances would be seen in the frequency spectrum of velocity and heat flux.

The turbulence field in the current work is also considerably different from that investigated in the previous study. The turbulence field of Holmberg was not what is believed to be representative of engine conditions. The turbulence intensity approaching the turbine blade cascade in the current experiments is almost three times greater, and the integral scale to blade chord ratio $\left(\Lambda_{\mathrm{x}} / \mathrm{c}\right)$ is between 0.13 and 0.16 , as compared to a maximum value of only 0.06 in the work of Holmberg. The desired $\Lambda_{\mathrm{x}} / \mathrm{c}$ value was estimated based on combustor turbulence measurements by Van Fossen and Bunker (2002). The value used in this work closely matches that reported by Van Fossen.

The work of Holmberg (1996) focused on a frequency domain analysis of surface heat transfer and freestream turbulence interactions. This work employs many of the tools used by the previous work, but focuses more on quantifying the effects of 
turbulence on heat transfer and developing an analytical model for explaining and predicting turbulent heat transfer.

This work reports the measurements of baseline (low turbulence) time-averaged heat transfer and the increases in heat transfer due to the generated freestream turbulence field on both the suction and pressure surfaces of the turbine blades at three locations. The results of time-averaged heat transfer measurements are presented in Section 3.2.3.

Time-resolved measurements of heat flux and velocity are also reported and analyzed. Analytical modeling of a mechanism by which freestream turbulence affects surface heat transfer is documented in Section 3.4. The analytical model development and application is detailed in both a "single-scale" and "multiple-scale" framework. The "single-scale" model (Section 3.4.1) utilizes time-resolved velocity data in the timedomain to determine model input parameters. The model is directly applied to the timeaveraged heat transfer results measured in the current work, as well as to results reported by other research groups. The "multiple-scale" model looks at both time-resolved velocity and heat flux data in the frequency domain (Section 3.4.4). The purpose of this application of the analytical model was to attempt to determine which frequencies (eddy scales) had the largest impact on surface heat transfer. 


\section{CHAPTER 2}

\section{EXPERIMENTAL SETUP}

\subsection{Transonic Cascade Wind Tunnel}

The experimental facility in which the majority of the research for this work was performed was the transonic cascade wind tunnel at Virginia Tech. The wind tunnel is a blowdown facility, which controls tunnel inlet total pressure to a desired value through a feedback control loop. The tunnel is capable of run times of up to 30 seconds with constant inlet pressure and heated flow. A schematic of the facility is show in Figure 2.1.

High-pressure air at approximately $1240 \mathrm{kPa}(180 \mathrm{psig})$ is supplied by a four stage, reciprocating compressor to two large storage tanks. Moisture and contaminants are removed from the compressed air by an activated alumina-drying unit. Between the facility and the supply pipe from the storage tanks are two automatic valves in series. The first valve is an open-close valve, which isolates the high-pressure air from the tunnel until the valve is opened. Once this valve is opened, air flows through the downstream valve, which is initially opened slightly awaiting a feedback pressure. The downstream valve will actuate based on feedback pressure measured by a total pressure probe at the test section inlet to maintain a constant inlet pressure. A PC based control program using both a feedback and feed forward algorithm allows the desired objective total pressure to be set and controls the actuation of the valve. Figure 2.2 shows a sample time history of tunnel inlet total pressure. To achieve the design Mach number and Reynolds number for the cascade, an inlet objective total pressure was set to a value of 69 $\mathrm{kPa}(10$ psig).

Prior to entering the test section, the tunnel flow passes through two bundles of copper tubing, which serve as a passive heat exchanger. The heat exchanger tube bundles are charged by a heat exchange loop built into the wind tunnel. Two valves, shown in Figure 2.1, are closed to isolate the tube bundles from the mainstream flow path. Air is 
heated by two $36 \mathrm{~kW}$ electric heaters and circulated through the heating loop by an axial fan at approximately $2 \mathrm{~m} / \mathrm{s}$, heating the copper tubes. The valves are closed prior to wind tunnel startup, restoring the path for mainstream airflow through the tunnel. The tunnel air passes over the copper tube bundles, which transfer heat to the mainstream flow. The air leaving the heat exchanger and entering the blade cascade test section can be heated as high as $120^{\circ} \mathrm{C}\left(248^{\circ} \mathrm{F}\right)$. Thermocouples and a total temperature probe are located within the heating loop and tube bundle to monitor the tube temperature during heating and the total temperature of the flow during a tunnel run, respectively. Due to the passive nature of the heat exchanger, the total temperature of the air decreases during a tunnel run as the tubes lose energy and transfer heat to the flow. As will be discussed later, heat transfer data reduction techniques take advantage of the transient nature of the flow temperature.

For experiments with high freestream turbulence, a passive turbulence grid is placed between the heat exchanger and the test section inlet (shown in Figure 2.1). The turbulence grid is briefly discussed in Section 2.4 and details of the design and testing of the grid are presented in Appendix A.

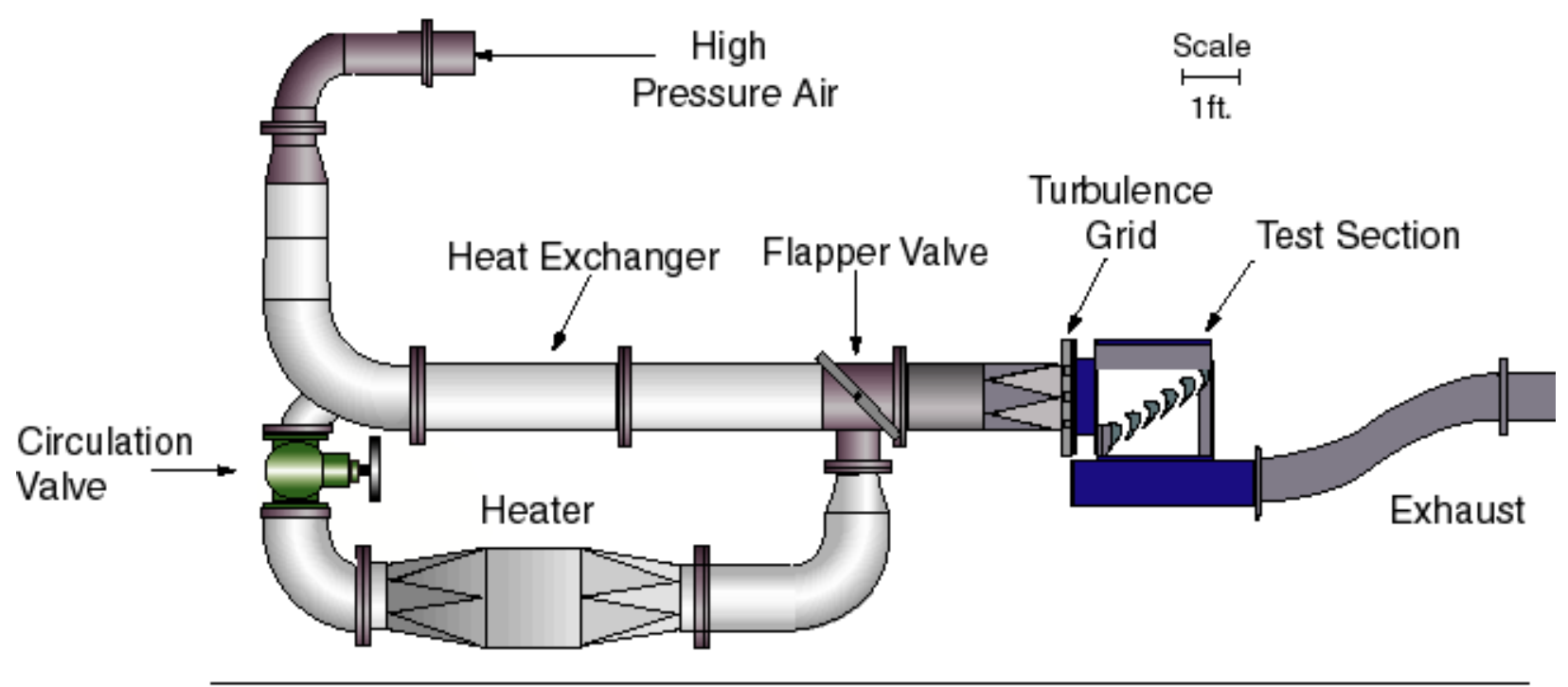

Figure 2.1: Virginia Tech transonic blowdown wind tunnel facility 


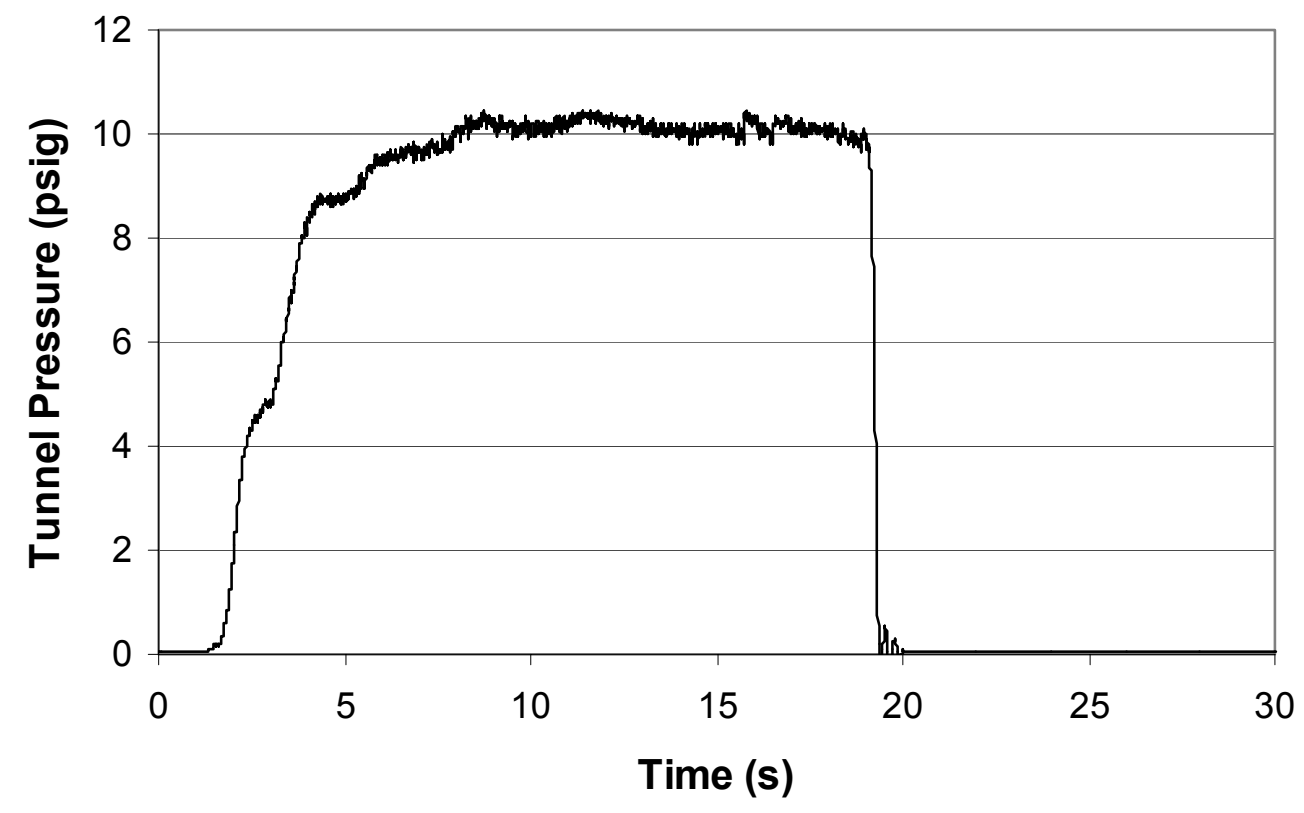

Figure 2.2: Typical tunnel inlet total pressure plot

\subsection{Cascade Test Section And Blade Instrumentation}

The turbine blade cascade test section used in the facility consists of 4 full and two half blades, creating a total of five flow passages. Figure 2.3 shows an illustration of the turbine cascade. The turbine blades are held in place by two Plexiglas end walls, allowing for shadowgraph or Schleiren flow visualization. The blade design is a high turning, generic GE first stage rotor geometry. The predicted Mach number profile for the blade is shown in Figure 2.4. The flow exits the test section and is exhausted to atmospheric conditions through a muffler. The non-dimensional conditions of the flow in the cascade are representative of operating conditions of modern gas turbine engines. The non-dimensional conditions and the cascade conditions and geometry are shown in Table 2.1 below. 
Table 2.1: Non-dimensional conditions and cascade conditions and geometry

\begin{tabular}{|l|c|c|}
\hline \multicolumn{1}{|c|}{ Non-dimensional Conditions } & Engine & Experiment \\
\hline Mach No. Exit & Transonic & 1.2 \\
Reynolds No. Inlet & $5 \times 10^{5}$ & $1 \times 10^{6}$ \\
Temperature Ratio $\left(\mathrm{T}_{8} / \mathrm{T}_{\mathrm{b}}\right)$ & 1.4 & 1.4 \\
\hline
\end{tabular}

\begin{tabular}{|l|c|}
\hline Cascade Conditions and Geometry \\
\hline Inlet Mach & 0.4 \\
Exit Mach & 1.2 \\
Inlet total pressure & $164 \mathrm{kPa}(10 \mathrm{psig})$ \\
Inlet total temperature & $100^{\circ} \mathrm{C}$ \\
Reynolds No. Exit $\left(\mathrm{Re}_{\mathrm{ex}}\right)$ & $5 \times 10^{6}$ \\
Blade aerodynamics chord $\left(\mathrm{c}_{\mathrm{a}}\right)$ & $13.6 \mathrm{~cm}$ \\
Suction surface chord $\left(\mathrm{c}_{\mathrm{ss}}\right)$ & $24.8 \mathrm{~cm}$ \\
Pressure surface chord $\left(\mathrm{c}_{\mathrm{ps}}\right)$ & $14.9 \mathrm{~cm}$ \\
Blade span $(\mathrm{sp})$ & $15.3 \mathrm{~cm}$ \\
Blade pitch $(\mathrm{p})$ & $12.2 \mathrm{~cm}$ \\
$\mathrm{sp} / \mathrm{c}$ & 1.12 \\
$\mathrm{p} / \mathrm{c}$ & 0.9 \\
\hline
\end{tabular}

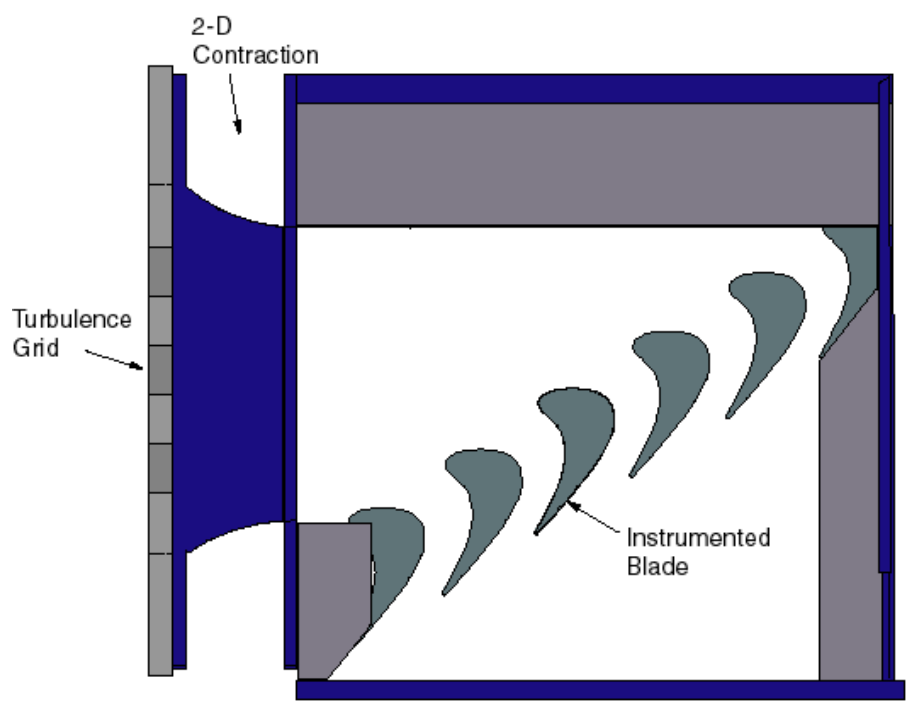

Figure 2.3: Cascade test section illustration showing instrumented blade location 


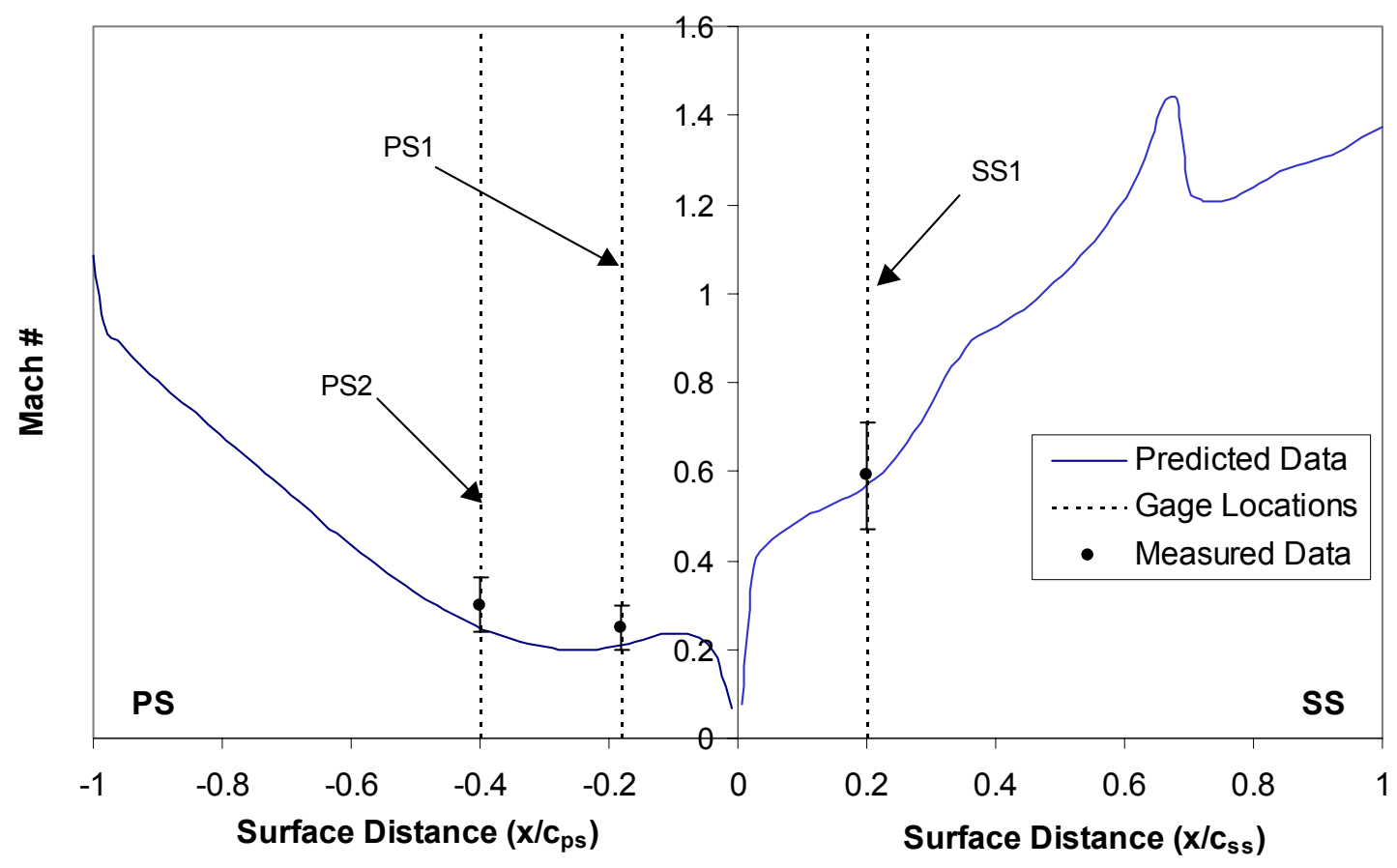

Figure 2.4: Predicted Mach number distribution for the turbine blades

One blade near the center of the cascade is instrumented on both the pressure and suction surfaces at roughly mid-span to avoid endwall boundary layer effects. The sensors were staggered in the spanwise direction to prevent small flow disturbances in the boundary layer caused by the gage mounting from effecting downstream gages. Measurements were made at locations on both the pressure and suction surfaces of the blade to compare the effects of turbulence on heat transfer under greatly different flow conditions (differences in mean velocity, pressure gradient and curvature and velocity fluctuations). For the results presented in this work, one location on the suction surface (SS1) was used and two locations on the pressure surface (PS1 and PS2). Each measurement location consists of an array of three sensors, as shown in Figure 2.5. Each triplet of sensors includes a Vatell HFM-7 heat flux microsensor, a Kulite XCQ-062-50a pressure transducer and a K-type thermocouple. The heat flux sensors and pressure transducers are discussed in Section 2.3. 


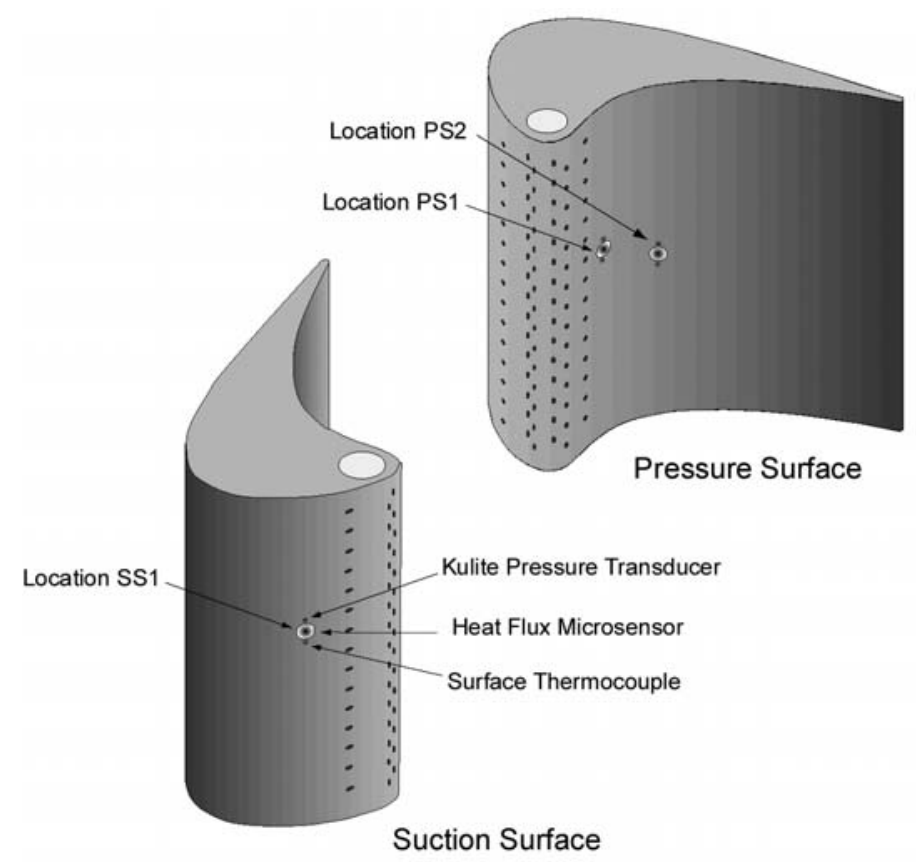

Figure 2.5: Turbine blade showing measurement locations and instrumentation details

The location of the gages on the two surfaces was selected such that the gages were in regions of high heat transfer and for ease of mounting. Since the gages are insert type, only regions of the blade where there is sufficient material for machining and mounting of the sensors could be instrumented. The instrumented blade was machined in two pieces such the blade could be split in half to mount the gages so that they are flush with the blade surface (Bubb, 1999). The suction surface gage (SS1) is located at a distance of $\mathrm{x} / \mathrm{c}_{\mathrm{ss}}=0.20$ from the stagnation point of the turbine blade. The two gages on the pressure surface of the blade (PS1 and PS2) are located at $\mathrm{x} / \mathrm{c}_{\mathrm{ps}}=0.16$ and $\mathrm{x} / \mathrm{c}_{\mathrm{ps}}=0.40$, respectively. The chord length of the blade on each surface is denoted as $\mathrm{c}_{\mathrm{ss}}$ for the suction surface and $\mathrm{c}_{\mathrm{ps}}$ for the pressure surface. Based on aerodynamic chord length $\left(\mathrm{c}_{\mathrm{a}}\right), \mathrm{SS} 1$ is located at $\mathrm{x} / \mathrm{c}_{\mathrm{a}}=0.36$, PS1 is at $\mathrm{x} / \mathrm{c}_{\mathrm{a}}=0.20$, and PS2 is at $\mathrm{x} / \mathrm{c}_{\mathrm{a}}=0.43$. The location of the gages relative to the Mach number distribution is shown in Figure 2.4. It should be noted that the two locations on the pressure surface are located in regions of relatively different curvature and pressure gradient. Table 2.2 summarizes the conditions at the gage locations. 
Table 2.2: Blade Measurement Locations

\begin{tabular}{|c|c|c|c|c|}
\hline Location & $\mathrm{x} / \mathrm{c}_{\text {ss or ps }}$ & $\mathrm{x} / \mathrm{c}_{\mathrm{a}}$ & $\begin{array}{c}\text { Approx. } \\
\text { Mach Number }\end{array}$ & $\begin{array}{c}\text { Pressure } \\
\text { Gradient (+/-) }\end{array}$ \\
\hline SS1 & 0.20 & 0.36 & 0.55 & + Favorable \\
\hline PS1 & 0.18 & 0.20 & 0.22 & - Adverse \\
\hline PS2 & 0.40 & 0.43 & 0.25 & + Favorable \\
\hline
\end{tabular}

\subsection{InSTRUMENTATION AND DATA ACQUISITION SYSTEMS}

In order to perform measurements for the analysis of turbulent heat transfer, instrumentation measuring both steady and unsteady mainstream flow and blade surface conditions was employed. Steady measurements were used to determine flow conditions within the turbine cascade such as inlet Mach number and Reynolds number, as well as steady heat transfer coefficients and static pressures on the blade surface. Unsteady measurements were performed to measure time-resolved velocity and heat flux data. Following is a detailed description of the instrumentation used in all experiments.

\subsubsection{Vatell HFM-7 Heat Flux Sensors}

Measurements of heat flux on the blade surface were performed using Vatell HFM-7 heat flux sensors. The heat flux microsensor (HFM) technology is documented in published papers by Holmberg and Diller (1995), Hager, et.al. (1991), and Baker and Diller (1993). Calibration techniques for the heat flux sensor are discussed in detail in Smith, et al. (1999). The heat flux microsensor consists of two sensors, one for measurement of heat flux (HFS) and a resistance temperature sensor (RTS). The sensor is an insert type gage that is designed to be inserted into a machined slot such that the sensor face is flush with the measurement surface. The sensor face is approximately 6.35 $\mathrm{mm}(0.25 \mathrm{in}$.) in diameter, which includes both the heat flux sensor footprint $(4.5 \mathrm{~mm})$ and the resistance temperature sensor. The gages are sufficiently small compared to the blade chord and span to provide reasonably good spatial resolution and the ratio of the turbulence integral length scale to the sensor diameter is approximately $\Lambda_{\mathrm{x}} / \mathrm{D}_{\mathrm{s}}=5.5$. Using the sensor diameter and a convective velocity of approximately $100 \mathrm{~m} / \mathrm{s}$, the frequency resolution of the gages is estimated as approximately $20 \mathrm{kHz}$. The heat flux sensors 
attenuate energy above this frequency. As illustrated in the results presented in the next chapter, this is well above the frequency range of interest, since there is no coherence between velocity and heat flux above $5 \mathrm{kHz}$. The scales of interest discussed later are all on the order of the gage diameter or larger. An illustration of the HFM-7 is shown in Figure 2.6a. The heat flux sensors are made on a substrate of aluminum nitride, which has thermal properties similar to those of aluminum, minimizing error in measurement of heat flux from a mismatch of materials.
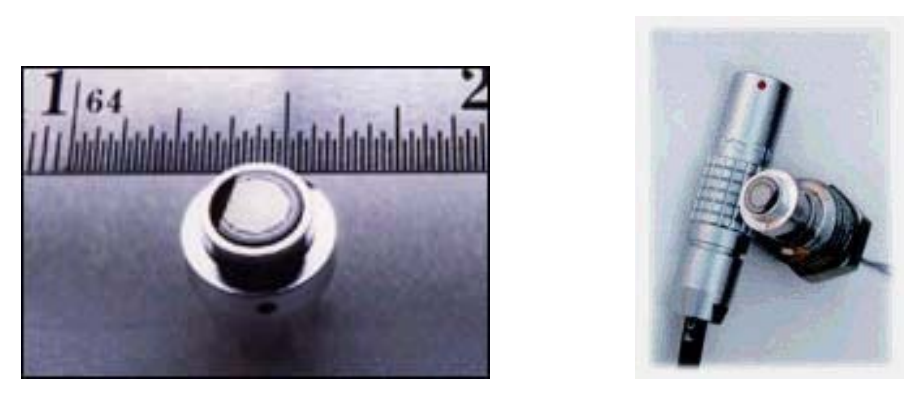

Figure 2.6a. Vatell HFM-7 Heat Flux Microsensor Source: Vatell Corporation website

The heat flux sensor (HFS) gives an output voltage that is linearly proportional to heat flux. The sensitivity of the HFS is approximately $150-200 \mu \mathrm{V} / \mathrm{W} / \mathrm{cm}^{2}$, depending on the gage. The HFS sensitivity includes a small correction based on gage temperature. The resistance temperature sensor (RTS) generates a voltage output with temperature changes by applying a $0.1 \mathrm{~mA}$ excitation to the resistance element on the sensor. The sensor resistance $\left(R_{0}\right)$ is measured when the gage is at thermal equilibrium with its surroundings and the voltage output is zeroed at this temperature. Measured voltages are converted to a resistance using Ohm's Law (V=IR) and the known excitation current and amplifier gain and are then added to the zeroed resistance $\left(\mathrm{R}_{\mathrm{o}}\right)$. The sensor temperature is then determined through a calibrated linear resistance/temperature curve for each gage, as shown in Equations 2.1 and 2.2.

$$
R=\frac{V_{R T S}}{I_{R T S} \cdot G_{R T S}}+R_{o}
$$




$$
T=c \cdot R+d
$$

where $V_{\text {RTS }}$ is the measured voltage output of the RTS sensor, $I_{R T S}$ is the applied excitation current $(0.1 \mathrm{~mA}), \mathrm{G}_{\mathrm{RTS}}$ is the RTS amplifier gain and $\mathrm{c}$ and $\mathrm{d}$ are the coefficients of the linear resistance/temperature curve for the gage. Heat flux from the measured HFS voltage signal was determined by:

$$
q^{\prime \prime}=\frac{V_{H F S} / G_{H F S}}{g \cdot T+h}
$$

where $V_{H F S}$ is the measured voltage from the HFS sensor, $G_{H F S}$ is the HFS amplifier gain and $\mathrm{g}$ and $\mathrm{h}$ are coefficients of the sensitivity of the sensor, accounting for gage temperature (T). The sensors are each connected to a Vatell AMP-6 amplifier, which provide the HFS and RTS gains and RTS excitation current. The gain for both the HFS and RTS was typically set to 100 , providing an easily measured signal with low signal-tonoise ratio. The output from the amplifier is connected to the data acquisition system through a BNC connection.

The heat flux sensors were used to perform measurements of both steady heat transfer coefficient and for time-resolved measurements of heat flux. For steady heat transfer measurements, output voltages from the sensor were recorded at $100 \mathrm{~Hz}$ sampling frequency and were sampled over the course of a 30 second tunnel run. For measurements of time-resolved heat transfer, data were recorded at $100 \mathrm{kHz}$ for a short duration of $1.3 \mathrm{~s}$ and filtered at $40 \mathrm{kHz}$. The sampling period gives a sufficient sample size for statistical analysis while limiting the output file sizes. Popp (1999) investigated the dynamic behavior of the HFM-7 gage in detail. It was demonstrated that the gage responds like a first order system with a time constant of $17 \mu \mathrm{s}$, or a frequency response of approximately $60 \mathrm{kHz}$. This provides a wide enough frequency band for sampling time-resolved heat flux data for correlation with high frequency velocity data.

\subsubsection{Kulite Pressure Transducers}

The pressure transducers used in this experiment were Kulite XCQ-062-50 highresponse miniature transducers (Figure 2.6b). The transducers were used to measure 
surface pressure to determine the Mach number near the blade surface. The transducers have a diameter of $1.7 \mathrm{~mm}(0.067 \mathrm{in})$ and are approximately $1 \mathrm{~cm}(0.4 \mathrm{in}$.$) in length.$ Each transducer is equipped with a B-screen, which protects the internal diaphragm from being damaged by debris moving through the test section. The sensor is also an insert type gage that is flush mounted with the measurement surface.

The Kulite sensors are located at the same chordwise locations as the heat flux sensors, but are slightly offset from the HFM gages in the spanwise direction, as shown in Figure 2.5. The lead wires for all of the Kulites are connected to a Measurements Group 2310 signal conditioner/amplifier via a bridge board. The 2310 amplifier provides the excitation voltage to the transducer and applies a gain to the output signal. The voltage output from the transducer is zeroed at ambient conditions such the transducer gives an output proportional to gage pressure (psig). The amplifier also has built in filter settings. For the experiments here, the gages were not used to perform time-resolved pressure measurements; therefore the frequency bandwidth of the transducers was not of importance and the amplifiers were set to "wide-band". The transducers are capable of high-frequency measurements of static pressure and have a frequency range of up to 25 $\mathrm{kHz}$.

The output voltages from all Kulites were reduced to gage pressure (psig) using a known sensitivity of each transducer. The sensitivity of the Kulites used for these experiments is typically between 2.0 and $4.0 \mathrm{mV} / \mathrm{psi}$. The general formula for reducing the Kulite data is as follows:

$$
P(p s i g)=\frac{V_{p}}{S_{p} \cdot G_{p}}
$$

where $V_{p}$ is the amplified output voltage measured from the transducer, $S_{p}$ is the sensitivity of the transducer, and $G_{p}$ is the gain setting on the amplifier. The gain for all pressure transducers used during experimentation was set to 100 . 


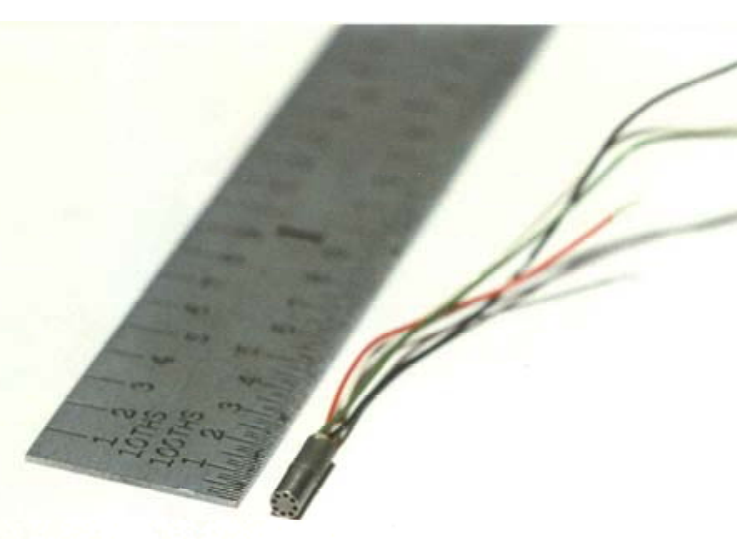

Figure 2.6b: Unmounted Kulite pressure transducer

\subsubsection{Hot-wire Probes and Anemometer}

\subsubsection{Probe Design}

Measurements of time-resolved velocity were performed with single sensor hotwire probes (straight wire). The probes were used to make measurements in the mainstream flow upstream of the cascade and in the turbine blade passages near the blade surface. Mainstream measurements were performed to characterize the turbulence field downstream of the grid (turbulence intensity and length scale) and to measure the decay of turbulence intensity and dispersion of length scale as the flow progresses through the test section, towards the cascade of blades. Passage measurements were performed to measure time-resolved velocity near the surface of the instrumented blade.

For mainstream flow measurements, a Dantec Type 55P71 hot-wire probe and several custom made hot-wire probes were used. The wire sensing area on all probes is an annealed tungsten wire with a diameter of $5 \mu \mathrm{m}$ and length between 1-1.5 mm. The probes are shown in Figure 2.7a and Figure 2.7b. Figure 2.7a details the dimensions of the custom made hotwires, while Figure $2.7 \mathrm{~b}$ shows a photograph of all wires used in the experiments. 


\section{Passage Hot-Wire (PHW) Probe}

Single U-wire probe for passage measurements

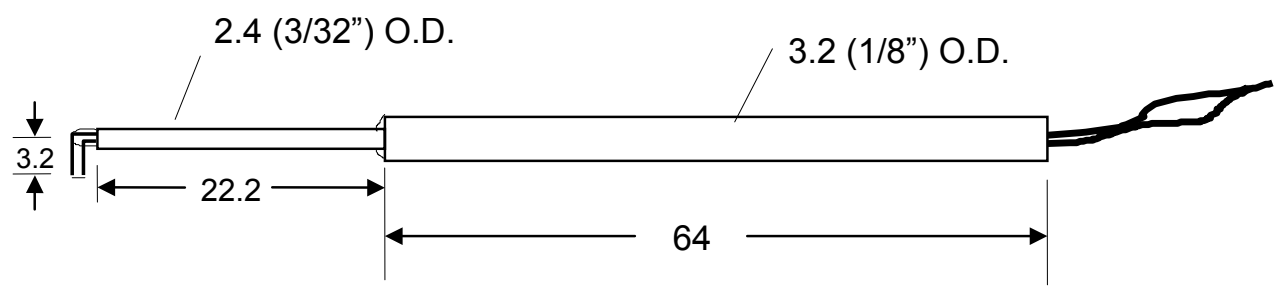

Upstream Hot-Wire (UHW) Probe

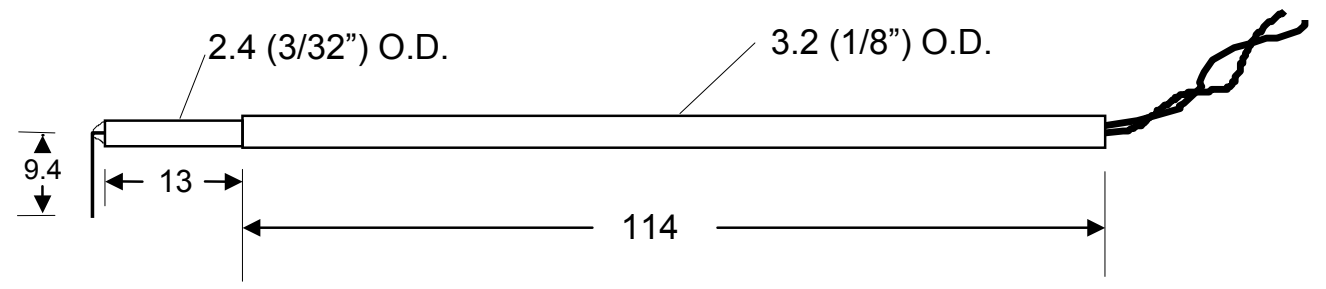

Dimensions are in $\mathrm{mm}$

Figure 2.7a: Drawings of custom hot-wire probes used in experiments

(Edited from Holmberg, 1996) 


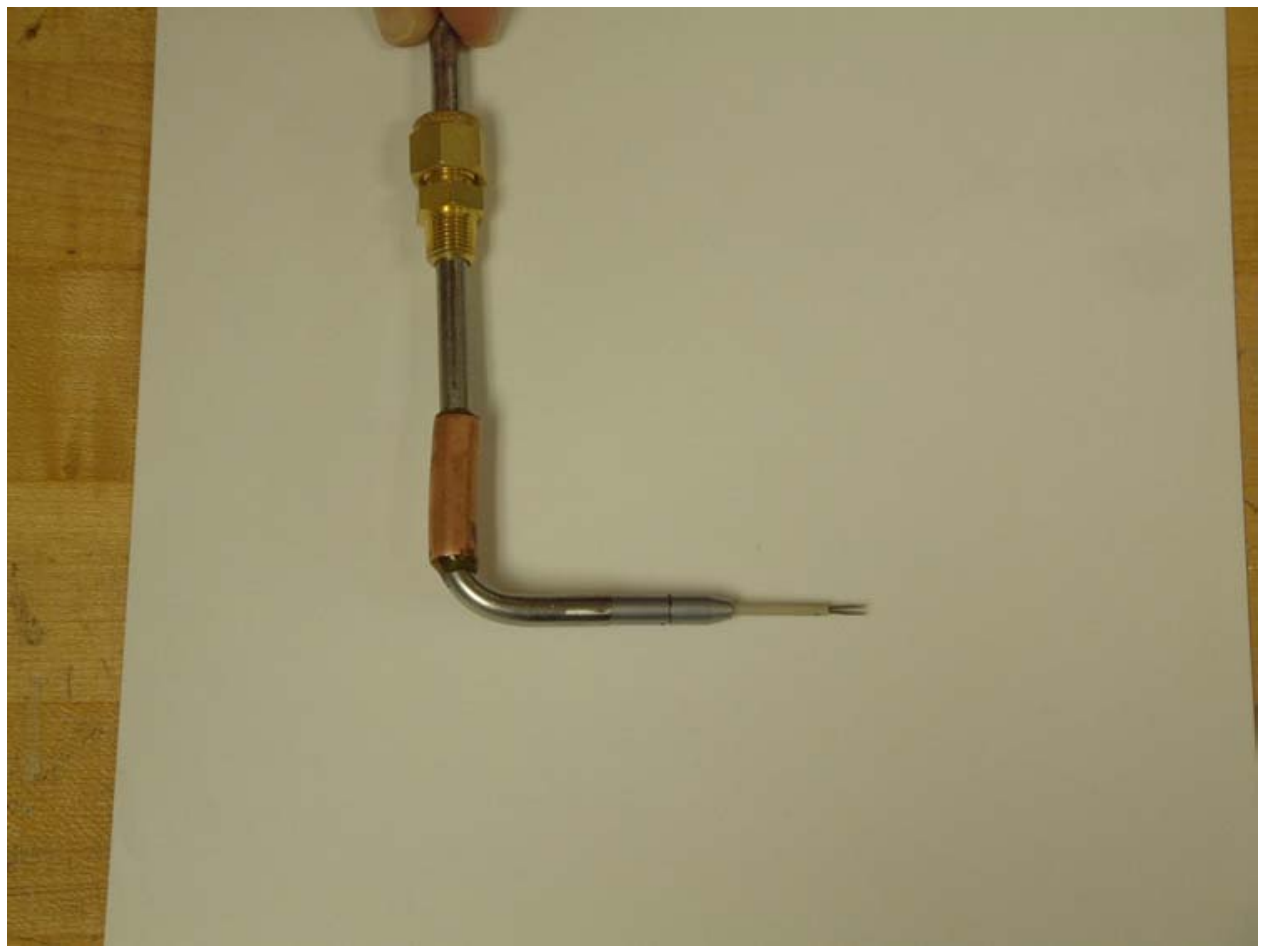

Dantec Hotwire

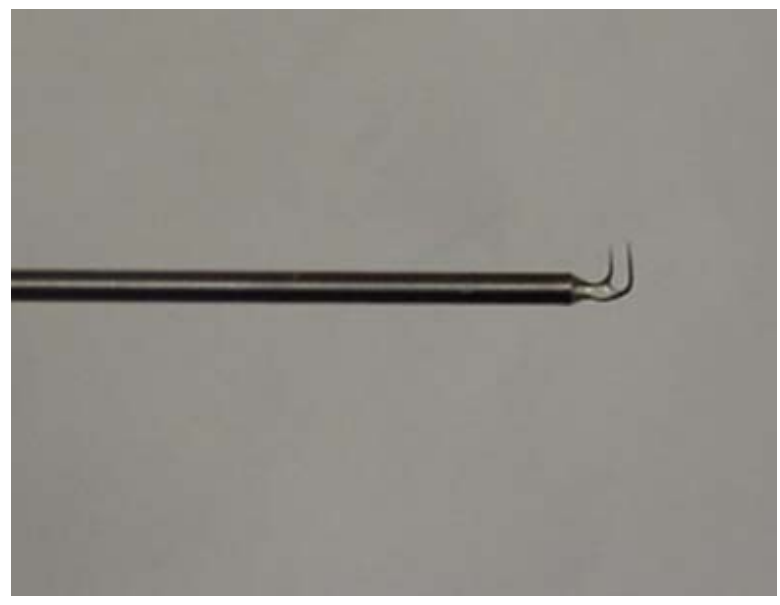

Passage Hot-wire (PHW)

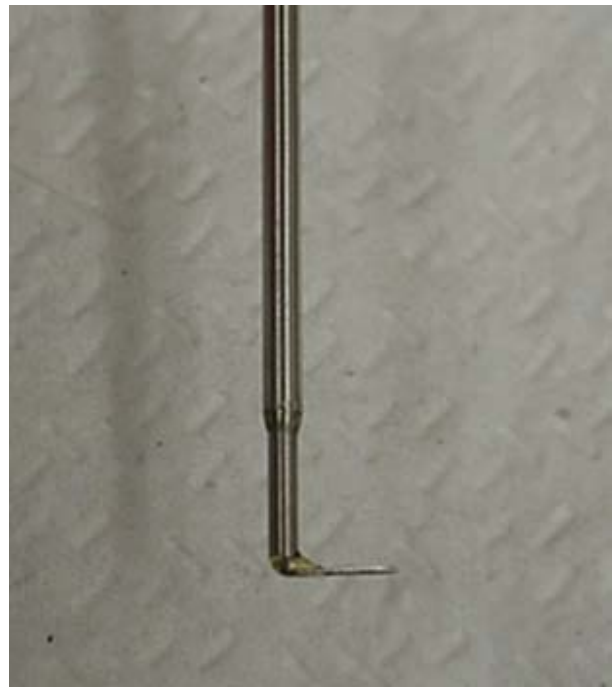

Upstream Hot-wire (UHW)

Figure 2.7b: Pictures of hot-wire probes used in experiments 
The Dantec hot-wire is mounted on a $90^{\circ}$ probe holder mounting so that the wire is oriented in the proper direction to measure streamwise velocity (U) and velocity fluctuations $\left(\mathrm{u}^{\prime}\right)$. The probe holder is mounted on the sidewall of the wind tunnel. The Dantec probes seemed to be more robust than the custom made probes, withstanding many runs at high velocity without breaking the wire. The disadvantage to this probe is that in order to move the probe to perform streamwise surveys one sidewall of the tunnel test section needed to be removed to move the probe mounting since the probe holder extends far upstream of where the probe is mounted. This wire was primarily used in low speed testing of the turbulence grid (see Appendix A). The probe was used for upstream measurements in the transonic wind tunnel prior to ordering the custom probes.

The custom made passage probes (PHW) were primarily designed for timeresolved velocity measurements above the surface of the turbine blades, but the probes were used for many of the mainstream flow measurements as well. The probes were custom made by Auspex Corporation (1416 Union Blvd., Allentown, PA 18109). The probe body was designed such that it would slip fit into a compression fitting for easy mounting on the sidewalls of the tunnel. As can be seen in Figure 2.7a, the prongs supporting the wire are at a $90^{\circ}$ angle to the probe body so that the wire could be oriented parallel to the surface of the turbine blade. The short length of the prongs allowed the wire to be moved in and out of the tunnel test section to different measurement locations without removing the sidewalls of the test section. The probe body extending from the mounting reduces down to a diameter of approximately $2.4 \mathrm{~mm}(0.093 \mathrm{in}$.) so that the probe body provides as little disturbance of the passage flow as possible. Figure 2.8 shows a picture of the UHW mounted such that the prongs are above the heat flux gage. In this configuration, the wire was approximately $5 \mathrm{~mm}$ above the surface of the turbine blade.

One final probe (UHW) that was used in the experiments was an Auspex probe (also shown in Figure 2.7a) with the prongs oriented $90^{\circ}$ from the probe body designed for vertical traverses behind the turbulence grid. The body of the probe was sized such that it would fit into a traverse mechanism mounted at the top of the turbine cascade upstream of the cascade passages. 


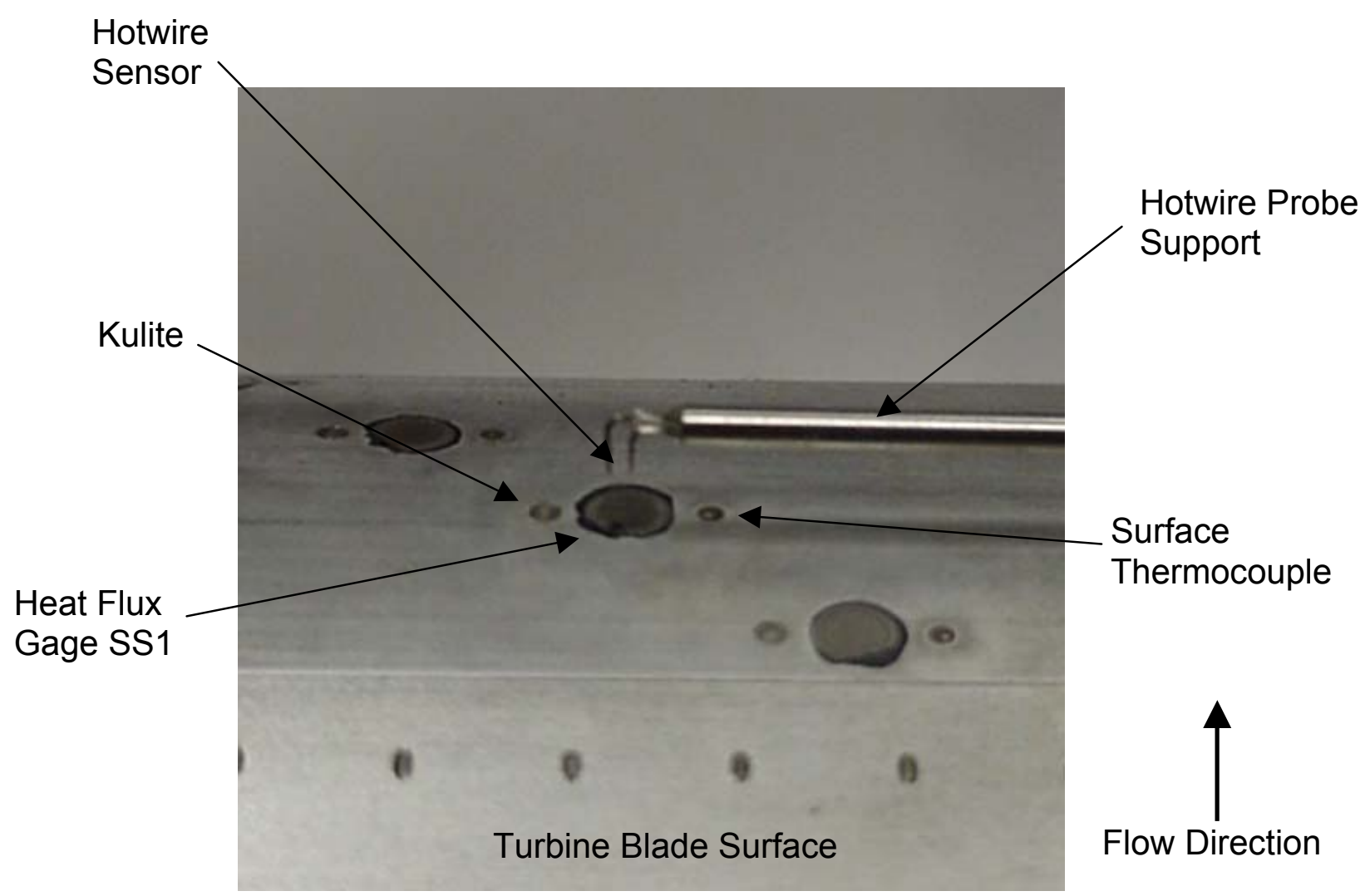

Figure 2.8: Picture of PHW probe mounted above heat flux gage on blade surface

Calibration of the hot-wire probes for use in the compressible flow field are described in Nix, et al. (2002) in Appendix A and in Holmberg (1996). Briefly, the wire is calibrated via a blowdown calibration method where the tunnel is run and allowed to blow down to low pressures. The cascade will unchoke and thus the Reynolds number will decrease with time as the tunnel inlet pressure drops. The wire is placed near a pitotstatic probe or surface pressure transducer to determine the flow conditions (static pressure and Mach number). The wire voltage output is measured; along with wire temperature and flow conditions, and a calibration curve is determined of the form:

$$
\mathrm{Nu}^{\prime}=C \cdot \operatorname{Re}_{w}^{x}
$$

where $\mathrm{Re}_{\mathrm{w}}$ is the wire Reynolds number, $\mathrm{Nu}$ ' is the wire Nusselt number and $\mathrm{C}$ and $\mathrm{x}$ are determined through a power law curve fit of the blowdown calibration data. Blowdown calibration was performed with the wire upstream of the cascade collocated with a pitotstatic probe and also at each of the three measurement locations near the blade surface. 
The wire calibrations for each of these four locations were combined to yield a single calibration curve. To minimize error due to calibration drift, the wire calibration was redone if significant time passed between calibration and sampling.

\subsubsection{Anemometer}

The hot-wire probes were controlled using a Dantec 55M01 hot-wire anemometer. The anemometer is used to set and maintain the wire resistance of the hotwire probe during testing and to tune the frequency response of the hot-wire. The overheat ratio of the hot-wire probes was typically set to between 1.5 and 1.8. Overheat ratios in this range provided frequency response up to approximately $50 \mathrm{kHz}$. The frequency response of the probe was tuned by applying a square wave to the probe and adjusting settings on the anemometer. The response of the wire to the square wave input was observed on an oscilloscope. The overheat ratio was held constant between calibration runs and experiments. The resistance of the probe assembly (cable, mounting support, probe body and wire prongs) was compensated for with the anemometer either through measurement of the resistance using a shorting probe, or, in the case of the custom probes, through manufacturer supplied data. The operating resistance of the hotwire was set based on reported probe resistance at a specified temperature (typically $\left.22^{\circ} \mathrm{C}\right)$.

\subsubsection{Low Frequency Data Acquisition- Labview}

Low frequency data acquisition was performed via a National Instruments ATMIO-16XE-50 digital 8 channel A/D board. The sampling capability of the data acquisition card is increased through the use of two National Instruments AMUX-64T multiplexer boards. Each multiplexer board has 32 channels, 16 channels for input via $\mathrm{BNC}$ cables, and 16 thermocouple input channels. Data is recorded through a LabView code designed to store data from 64 channels with the option of displaying up to 8 channels immediately after acquiring data. Due to the use of the multiplexer, the maximum sampling frequency is limited. Since the data acquisition system is for low frequency or "steady" sampling, the limitations of sampling frequency were not an issue. Data was typically sampled at $100 \mathrm{~Hz}$ for a 30 second tunnel run. The data acquisition system was manually triggered at the start of every run. Data sampled with the low speed 
data acquisition system included all pressures (tunnel total pressure, wall static pressure, data from test section pitot-static probes and blade surface pressures), tunnel total temperature and blade surface temperature, heat flux (HFS) and surface temperature (RTS) data from the HFM-7 sensors and surface thermocouples during steady heat transfer tests and hot-wire data during blowdown calibration of the hot-wires.

\subsubsection{High Frequency Data Acquisition - LeCroy}

High frequency data acquisition was performed using a LeCroy 6810 waveform recorder. The LeCroy module used in this work consists of four single channel modules, each with the capability of sampling data up to $1 \mathrm{MHz}$. For most high frequency data sets, the sampling frequency was set at $100 \mathrm{kHz}(\Delta \mathrm{t}=10 \mu \mathrm{s})$ and data were sampled for 1.31 seconds (131,070 data points per sample). The frequency resolution of the sampled data was then $\Delta \mathrm{f}=0.763 \mathrm{~Hz}$. Data were filtered at $40 \mathrm{kHz}$. The voltage limits and offsets were set on the LeCroy to maximize dynamic resolution of the measured signals. The data acquisition was triggered manually once the tunnel had reached a steady inlet pressure. The high speed data acquisition system was used to sample all hot-wire data (except during blowdown calibration) and high frequency surface heat flux from the HFM sensors.

\subsection{TurbulenCe Grid}

Turbulence was generated in the facility using a passive turbulence grid placed upstream of the cascade test section inlet (see Figure 2.1 and Figure 2.3). The grid (shown in Figure 2.9) consists of three $5.08 \mathrm{~cm}(2$ in.) wide bars spaced $7.62 \mathrm{~cm}(3 \mathrm{in}$.) apart. Each bar is $2.54 \mathrm{~cm}(1 \mathrm{in}$.) thick. 


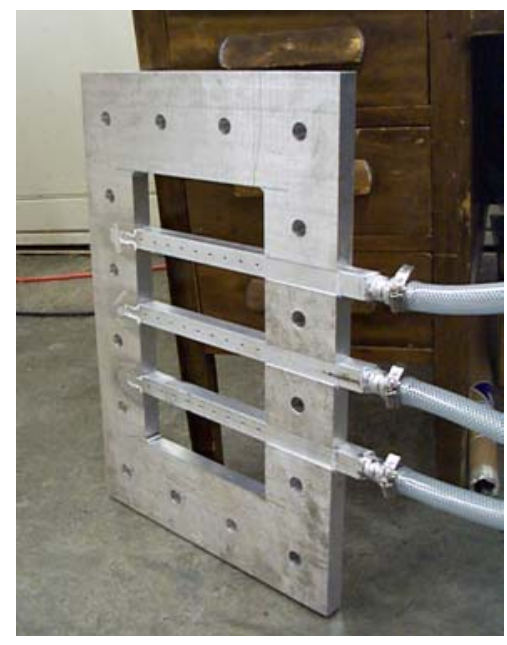

Original Design: Active Grid

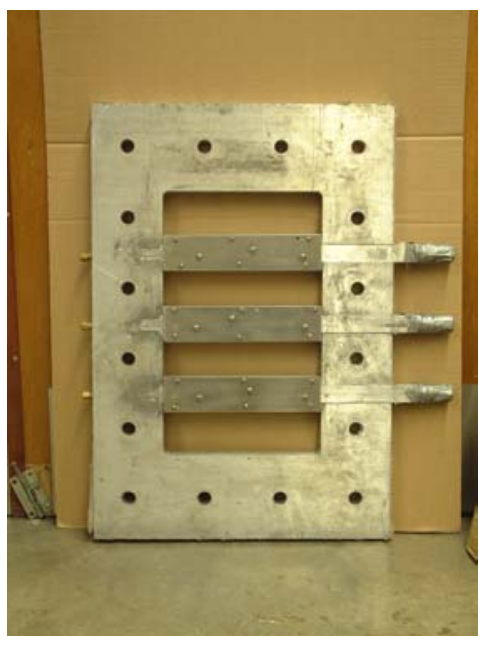

Modified Design: Passive Grid

Figure 2.9: Turbulence generating grid used in the facility

The grid was developed and evaluated through extensive testing in both the transonic facility and a low speed flow facility. Details of the development of the grid are discussed in Nix, et al. (2002) and the attached corresponding presentation (which extends the data presented in the paper) in Appendix A. The grid was originally designed to generate turbulence through active blowing. Testing of the active grid in the low speed facility produced the desired intensity (approximately 20\%) and scale of turbulence (3-4 $\mathrm{cm})$. The objective turbulence intensity and length scale were based on data reported by Van Fossen and Bunker (2002) and Radomsky and Thole (1998). Van Fossen and Bunker (2002) used a $60^{\circ}$ section of a GE 90 dual-annular combustor in a wind tunnel to model the exit flow of a combustor, including inlet swirl vanes, film cooling holes and dilution holes. All three of these combustor components contribute strongly to the combustor exit turbulence conditions. Hot wire surveys were performed downstream of the combustor section to determine turbulence intensity and length scale. Turbulence intensities as high as $20 \%$ were measured with length-scales between 1 and $1.5 \mathrm{~cm}$. Using these results, the desired length scale was increased to match the length scale to chord length ratio $\left(\Lambda_{x} / \mathrm{c}\right)$ of the blades of a GE 90 engine to the blades used in the transonic turbine cascade facility. The desired turbulence intensity was based on these results and reported decay data through a turbine vane passage by Radomsky and Thole 
(1998). The turbulence intensity of the core flow believed to be consistent with engine conditions based on these two works is in the range of $6-10 \%$.

Results of grid evaluation in the low speed rig and in the transonic facility are presented in detail in Nix, et al. (2002). When the turbulence grid was moved to the transonic facility, testing showed that the active grid was generating inadequate levels of turbulence (only about 5\%). It was desired to match the momentum flux ratio $\left(\mathrm{I}=\rho_{\mathrm{j}} \mathrm{u}_{\mathrm{j}}{ }^{2} / \rho_{\infty} \mathrm{u}_{\infty}{ }^{2}\right)$ of the blowing from the bars between the two facilities in order to obtain similar turbulence fields. The mainstream flow velocity $\left(\mathrm{u}_{\infty}\right)$ of the low speed facility was only $20 \mathrm{~m} / \mathrm{s}$ and the test section was at ambient conditions. Due to the high density $\left(\rho_{\infty}\right)$ and velocity $\left(\mathrm{u}_{\infty}\right)$ of the mainstream flow in the transonic facility, the pressure required within the internal plenum of the blowing bars to match momentum flux ratio with the low speed testing was much higher than achievable. The momentum flux ratio at the maximum plenum supply pressure to the bars in the transonic facility was 10-12 times lower than the low speed testing. At this momentum flux ratio, low speed test demonstrated that the blowing from the bars essentially only acted to fill in the wake from the bar, reducing the effectiveness of the grid in producing elevated levels of turbulence.

The grid was modified to be a passive grid with relatively large blockage area (approximately 40\%). The modification was made based on results obtained by Polanka and Bogard (1999) using large bars to generate high turbulence. The modified grid operated as a passive grid generated an acceptable level of turbulence with intensity of $15 \%$ and $2 \mathrm{~cm}$ length scale near the cascade test section inlet. The turbulence intensity decays to approximately $10 \%$ near the entrance to the cascade passages with a length scale of $2 \mathrm{~cm}$. This level is representative (if not slightly higher) than the levels believed to exist in the core flow following the first stage vane row.

One major concern with the modified grid was uniformity of the flow downstream of the grid. The modified grid has an open area of $60 \%$, with $40 \%$ flow blockage area, and thus there was concern with inlet flow non-uniformity due to large bar wakes. Crossstream traverses of both a total pressure probe and the upstream hot-wire probe (UHW) were performed behind the turbulence grid, approximately $10.2 \mathrm{~cm}$ (4 in.) upstream of the leading edge of the center cascade passage in order to measure flow and turbulence 
field uniformity behind the modified grid. Each traverse covered about half the passage in the pitchwise direction. The results of the surveys are shown in Appendix A. Total pressure surveys demonstrate that although there is a significant drop in total pressure across the grid (approximately 7\%), the total pressure of the flow approaching the leading edge of the cascade is relatively uniform, with total pressure uniformity of $\pm 1 \%$. The main tunnel flow passes through a 2:1 area contraction downstream of the grid (as shown in Figure 2.3) before entering the cascade test section, which is believed to help in merging and mixing of the relatively large bar wakes, thus increasing flow uniformity.

Also of concern was the possibility of vortex shedding off of the bars generating coherent structures in the flow. Analysis of the power spectral density (PSD) of the hotwire signals at various locations in the flow (varying in both streamwise and pitchwise location) demonstrated that the spectra were free of peaks which would indicate vortex shedding. The vortex shedding frequency of the bars as estimated based on a Strouhal number of 0.13 (versus 0.21 for circular cylinders). Estimating the expected frequency provided a guideline of where to look for evidence of shedding in the spectra. Plots of streamwise and spanwise spectral data are shown in the presentation in Appendix A.

\subsection{MeAsurement UnCERTAinty}

Measurement uncertainty in the current work is detailed in Appendix B. Table 2.3 below summarizes the total uncertainty determined for all relevant parameters in this work.

Table 2.3 Summary of total uncertainty $(\Delta)$

\begin{tabular}{|c|c|}
\hline Parameter & Total Uncertainty \\
\hline $\mathbf{h}$ & $6.3 \%$ \\
(average of low and high turbulence) & \\
\hline $\mathbf{u}^{\prime}{ }_{\text {rms }}$ & $5.0 \%$ \\
\hline Tu & $5.5 \%$ \\
\hline$\Lambda_{\mathbf{x}}$ & $6.1 \%$ \\
\hline$\Delta \mathbf{h}_{\mathbf{t}}$ & $4.5 \%$ \\
\hline
\end{tabular}




\section{CHAPTER 3}

\section{DATA ANALysis AND Results}

\subsection{TurbulenCE}

\subsubsection{Definition of Turbulence}

Turbulence, as defined by Hinze (1975), is an "irregular condition of flow in which the various quantities show a random variation with time and space coordinates, so that statistically distinct average values can be discerned." Further, it is stated that, when viewing an oscillogram (or time history of velocity for instance), "given a point in a turbulent domain a distinct pattern is repeated more or less regularly in time." If different turbulent fields are compared, there may exist a difference in the size (or amplitude) of the patterns, as well as the "violence" of the fluctuations. The author states:

"...to describe a turbulent motion quantitatively, it is necessary to introduce the notion of scale of turbulence..." and

"It is apparent that it is insufficient to characterize a turbulent motion by its scale alone, since to do so does not tell us anything about the violence of the motion. One cannot take the average value of the velocity as a measure of this violence, because the violence of the fluctuations with respect to this average velocity is just what one wants to know."

These two quantities, the turbulence intensity (violence of motion) and turbulence scale (scale of motions) can be determined through statistical and spectral analysis of a time-resolved measurement of velocity. Experimentally, this is typically measured through intrusive measurements such as hot-wire anemometry or high frequency pressure measurement devices, or by non-intrusive measurements such as LDV (Laser Doppler Velocimetry). The following sections will discuss how turbulence is characterized experimentally and will detail the analysis tools employed to do so. 


\subsubsection{Characterizing Turbulence}

\subsubsection{Time-Resolved Velocity Signal Decomposition}

In the current work, time-resolved velocity is measured using a single sensor hotwire probe. The hot-wire probe is limited in its measurement of turbulence by the frequency response of the probe and spatial averaging due to a finite length sensing wire. The hot-wire probe produces a time-varying voltage signal which can be converted to a streamwise velocity signal through calibration of the wire in a known flowfield (see Nix, et al., 2002 in Appendix A). From this velocity signal, one can determine the mean velocity $(\bar{U})$ and the fluctuating component of velocity $\left(u^{\prime}\right)$. The measured value of velocity at any given sample time $(\tilde{u})$ can be decomposed as follows (Sauer, 1996):

$$
\widetilde{u}=\bar{U}+u^{\prime}
$$

An example of a time varying signal and illustration of the components of the velocity signal is shown in Figure 3.1.

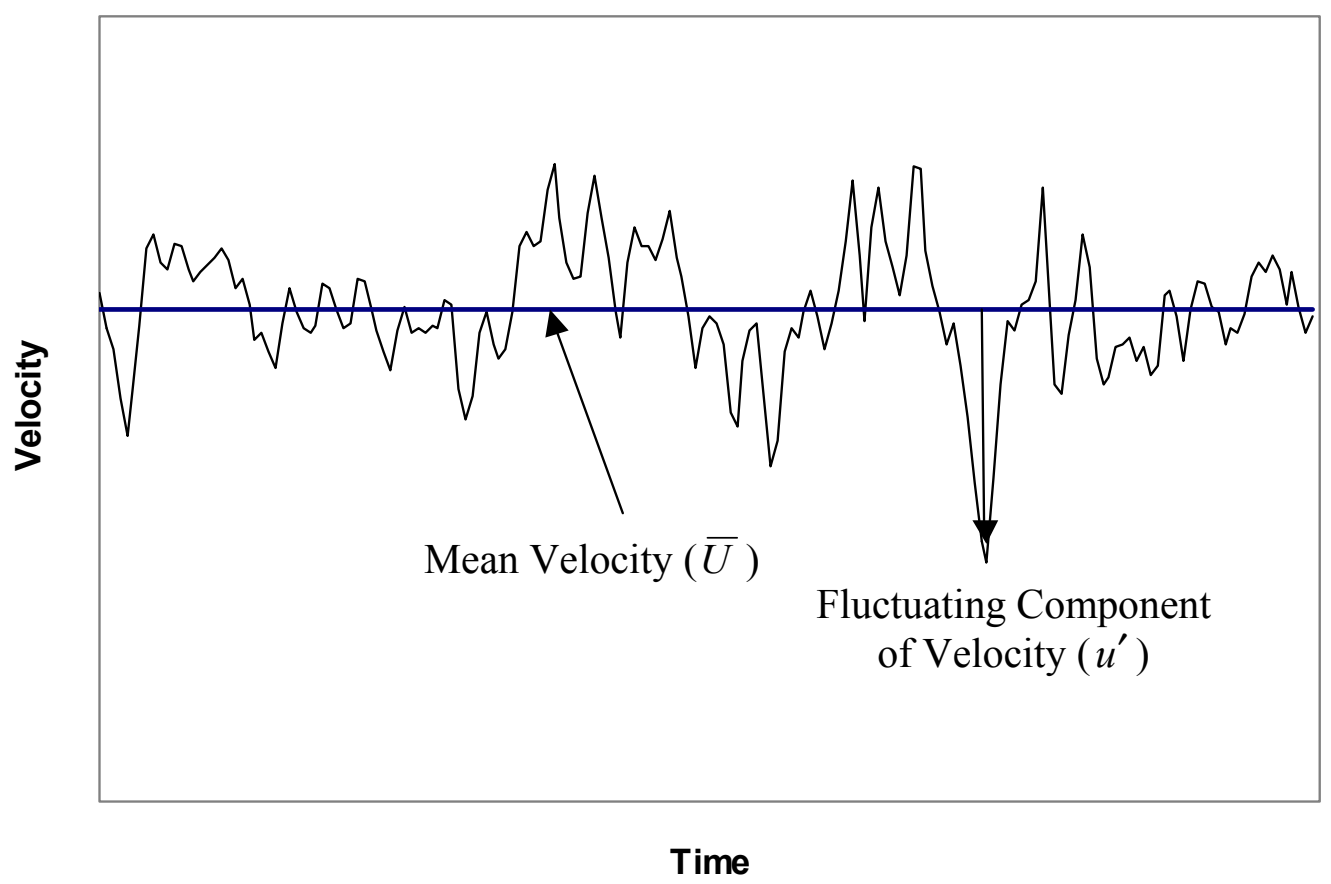

Figure 3.1: Illustration of velocity time signal and decomposition 
The mean velocity is determined by:

$$
\bar{U}=\frac{1}{T} \int_{t=0}^{T} \tilde{u} d t=\frac{1}{N} \sum_{i=1}^{N} \tilde{u}_{i}
$$

where $\mathrm{N}$ is the number of discretized data points in the sampling period of length $\mathrm{T}$. The fluctuating component of velocity is then determined by:

$$
u^{\prime}=\tilde{u}-\bar{U}
$$

\subsubsection{Turbulence Intensity and Velocity Spectrum}

From the decomposition of the turbulent velocity time signal, the turbulence intensity can be determined. Using the mean and fluctuating velocity components of the signal, the turbulence intensity $(\mathrm{Tu})$ of the flow at a given point is calculated by:

$$
T u=\frac{\sqrt{\overline{u^{\prime 2}}}}{\bar{U}}=\frac{u_{R M S}^{\prime}}{\bar{U}}
$$

The value of $u_{R M S}^{\prime}$, the root mean square (RMS) for the discretized fluctuating velocity signal, can be determined in either the time domain, or the frequency domain. Mathematically, this quantity is determined in the time domain as follows:

$$
u_{R M S}^{\prime}=\sqrt{\frac{1}{N} \sum_{i=1}^{N} u_{i}^{\prime 2}}
$$

Another term, the heat flux intensity $\left(\mathrm{Tu}_{\mathrm{q}}\right)$, can be defined in a similar way, using the time-resolved heat flux signal instead of the velocity signal.

In the frequency domain, the RMS can be determined through the power spectral density (PSD) of the fluctuating velocity signal $\left(u^{\prime}(t)\right)$. The velocity spectrum (PSD) is essentially the energy at each frequency of turbulent fluctuation (or eddy scale). The 
integral of this spectrum gives the fluctuating energy. Based on the discretized signal, this is the summation of the energy over the entire spectrum, which is equal to the square of the RMS of the velocity signal. In the frequency domain, the RMS is then determined as follows:

$$
u_{R M S}^{\prime}=\left[\int_{0}^{\infty} E(f) d f\right]^{1 / 2}=\left[\sum_{i=1}^{N} E(f)_{i} \cdot \Delta f\right]^{1 / 2}
$$

where $E(f)$ is the energy at each frequency and $\Delta f$ is the frequency resolution, which is equal to the sampling frequency divided by the number of sample points (N). The PSD is determined by:

$$
E(f)=\frac{S_{x x}}{\Delta f}=\frac{2 \cdot X(f) \cdot X(f)^{*}}{\Delta f \cdot N^{2}}
$$

where $\mathrm{S}_{\mathrm{xx}}$ is the auto-spectrum, $\mathrm{X}_{\mathrm{f}}$ is the FFT of the fluctuating velocity time-signal and the * denotes the complex conjugate of the FFT. The factor of $\mathrm{N}^{2}$ is a product of the FFT function in the Matlab software used to process the data.

The PSD of a measured turbulent velocity signal reveals much about the flow. Through observation of the PSD of one velocity signal compared to the PSD of a signal from a flow with different turbulence conditions, assuming they have similar mean velocities, one can easily determine which has the higher intensity and in some cases, larger or smaller scale (scale will be discussed in the next section). This is illustrated in Figure 3.2. The data in Figure 3.2 (as well as Figure 3.3 and Figure 3.4 later) is from the low speed testing of the turbulence grid. These plots are merely for illustration of the data analysis methods. In the figure, the two spectra are measured at different streamwise locations in a turbulent flow using identical sampling frequencies where the mean velocity is relatively constant. It is apparent from the figure that the turbulence level has decayed from signal 1 to signal 2 as integrating under the PSD curve of signal 1 will 
yield a higher value of $u_{R M S}^{\prime}$ than integrating under the PSD of signal 2. With similar mean velocity, this then indicates that signal one has higher turbulence intensity.

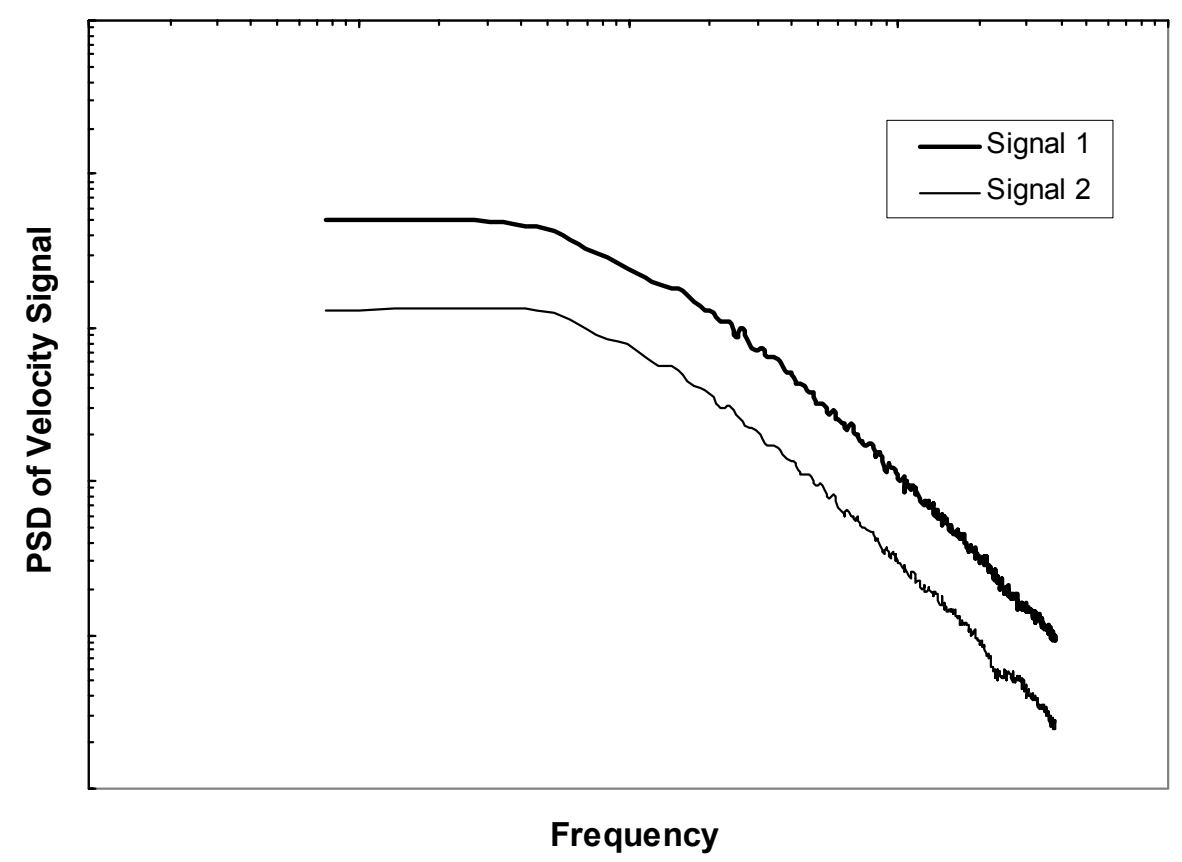

Figure 3.2: Example of velocity power spectral density (PSD)

\subsubsection{Length Scale and Autocorrelation}

The length scale of the turbulent flowfield used in this work is based on the integral length scale $\left(\Lambda_{\mathrm{x}}\right)$. The integral length scale is representative of the size of the most energetic eddies in the flow and is determined through analysis of the autocorrelation of the fluctuating velocity time signal. The autocorrelation $\left(\mathrm{R}_{\mathrm{uu}}\right)$ of the fluctuating component of velocity (normalized such that $R_{\text {uu }}$ is equal to 1 at zero lag, $\tau=0$ ), was calculated by:

$$
R_{u u}(\tau)=\left(\frac{\overline{u^{\prime}(t) \cdot u^{\prime}(t+\tau)}}{u_{R M S}^{\prime}{ }^{2}}\right)=\frac{1}{N}\left(\frac{\sum_{i=1}^{N} u_{i}^{\prime} \cdot u_{i+j}^{\prime}}{u_{R M S}^{\prime}{ }^{2}}\right) \text { where } \tau=j \cdot \Delta t
$$

In the limit as $\tau$ goes to zero (zero lag time), the value of the unnormalized autocorrelation is equal to the square of the RMS of the velocity signal. The 
autocorrelation was used to determine the integral time scale $(T)$ by integrating under the curve to the first zero crossing:

$$
T=\int_{t=0}^{\infty} R_{u u}(\tau) d \tau \cong \sum_{i=1}^{N_{0}} R_{u u i} \cdot \Delta \tau
$$

where $\mathrm{N}_{0}$ is the point of the first zero crossing.

Theoretically, the curve should be integrated to infinity (all data in the discretized time signal), however, in practice it is typically only integrated out to the first zero crossing, since noise may be present in the auto-correlation. This noise is an effect of dominant peaks in the frequency spectrum, in the case of this work from small amplitude, high frequency vibrations of the hot-wire probe support. Integrating the autocorrelation out beyond the first zero crossing may inflate the value of the time scale, even if the oscillations are small. From analysis of the autocorrelation of most measurements in this work, the oscillations in the "tail" of the autocorrelation (after the first zero crossing) are of very small amplitude. If there were a problem with the measurements (excessive probe vibrations or $60 \mathrm{~Hz}$ electrical noise for example), the autocorrelation would show significant oscillations in the decaying region and possibly large amplitude oscillations in the tail.

The streamwise integral length scale, which is representative of the largest eddies in the turbulent flowfield, is determined by invoking Taylor's hypothesis of frozen turbulence which gives:

$$
\Lambda_{x}=\overline{\mathrm{U}} \cdot T
$$

the time scale is multiplied by the mean velocity to yield the scale of the most energetic eddies in the flow.

Figure 3.3 shows two typical velocity autocorrelation curves. Assuming that the mean velocity is constant between the two curves, it can often be determined from comparison of two autocorrelations which signal has the larger scale. A slower decay indicates that correlation exists within the flow for larger time delays, and thus flow structures (e.g. turbulent eddies) are relatively larger. This function helps describe time 
scales (and length scales) present in the turbulent flowfield. In Figure 3.3, integrating under the autocorrelation curve for both signals indicates that signal 1 has a larger time scale than signal 2. Since the mean velocity for both signals is the same, the figure indicates that signal 1 has a larger length scale than signal 2.

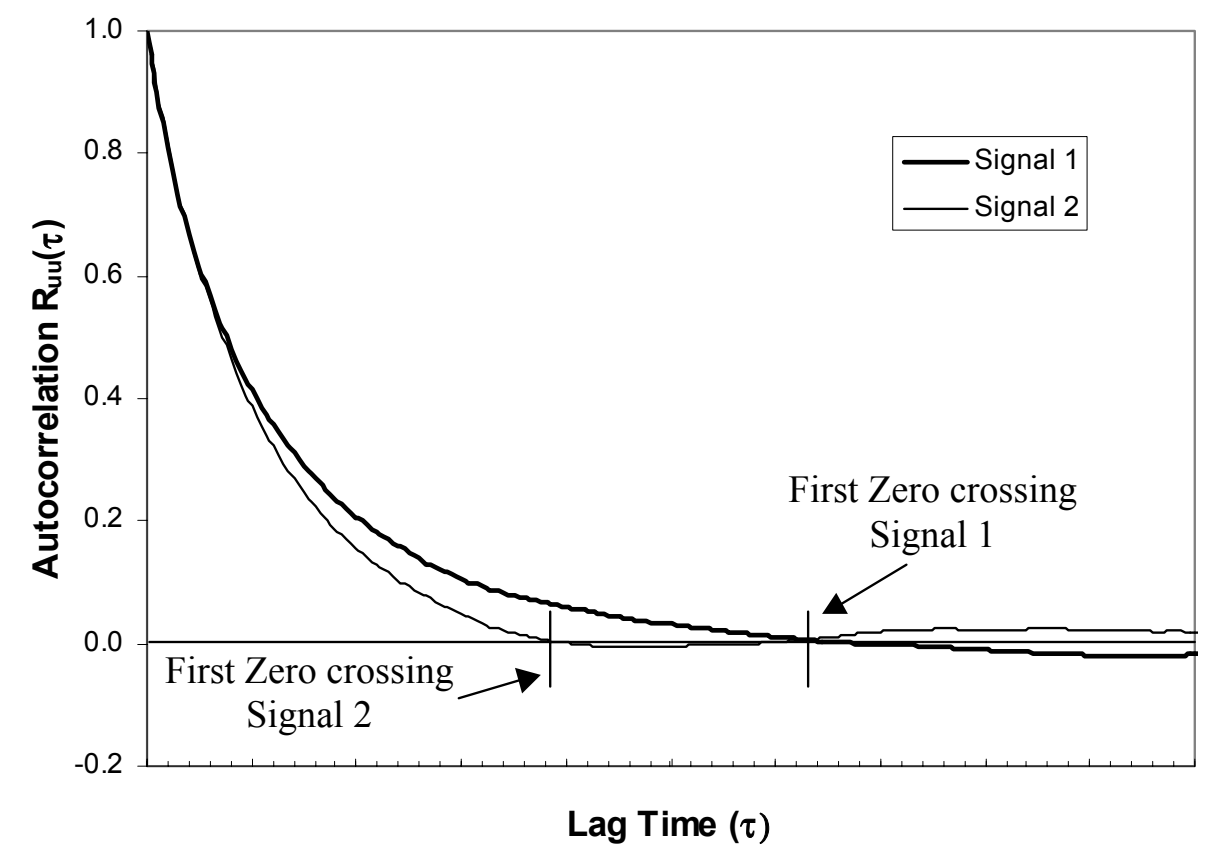

Figure 3.3: Typical autocorrelation curves $\left(\mathbf{R}_{\mathrm{uu}}\right)$

The function used to determine the autocorrelation (Equation 3.8) and time scale (Equation 3.9) can also be used to determine the time scale of the measured time-resolved heat flux signal by substituting q' for $u^{\prime}$. Usefulness of the heat flux time scale will be discussed later. A similar function can also be used to obtain time domain correlation (cross correlation) between two time-resolved signals (velocity and heat flux for instance).

\subsubsection{Energy Spectra Relations and Isotropic Turbulence}

As was indicated earlier, the PSD can also be used to get an estimate of the integral length scale. Referring to Figure 3.2, the turbulent spectrum is shown to be relatively flat in the low frequency range. At a certain frequency, the spectrum begins to slope downwards and tail off at higher frequencies. The region where the curve begins to 
slope is the approximate frequency corresponding to the most energetic eddies in the flowfield. As will be shown below, this frequency can be related to the integral scale.

It is often convenient to look at the turbulent velocity spectra in a different way. Grid generated turbulence, in the absence of outside forces, will tend towards isotropy. Isotropic turbulence is characterized by a cascade of energy from the larger eddies to the smaller eddies until the energy is viscously dissipated from the smallest scales (highest frequencies). The spectrum of isotropic turbulence is typically represented by the Von Kármán relation for one-dimensional spectra (Hinze, 1975):

$$
\frac{E(f) \cdot \bar{U}}{u_{R M S}^{\prime} \cdot \Lambda_{x}}=4 \cdot\left(1+\left(\frac{8 \pi f \cdot \Lambda_{x}}{3 \cdot \bar{U}}\right)^{2}\right)^{-5 / 6}=4 \cdot\left(1+\left(\frac{\kappa}{\kappa_{e}}\right)^{2}\right)^{-5 / 6}
$$

where the wave number, $\kappa$, is equal to $2 \pi f / \bar{U}$ and the most energetic wave number, $\kappa_{\mathrm{e}}$ is equal to $3 / 4 \Lambda_{x}$. Figure 3.4 shows an example of a normalized turbulent spectrum compared to the theoretical Von Kármán spectrum. From the raw, un-normalized spectrum (Figure 3.2), the estimated frequency (f) where the spectrum tails off (most energetic frequency) can be converted to the integral scale by $\Lambda_{x}=\frac{3 \cdot \bar{U}}{8 \pi f}$. In Figure 3.4, this frequency can be seen in the normalized spectrum where $\kappa \cdot \Lambda_{x} \approx 3 / 4$.

All experimental turbulence data was normalized and compared to the theoretical Von Kármán spectrum during data analysis to verify that the flow was behaving as expected. The tools presented in the preceding sections will be applied frequently throughout the course of this work. 


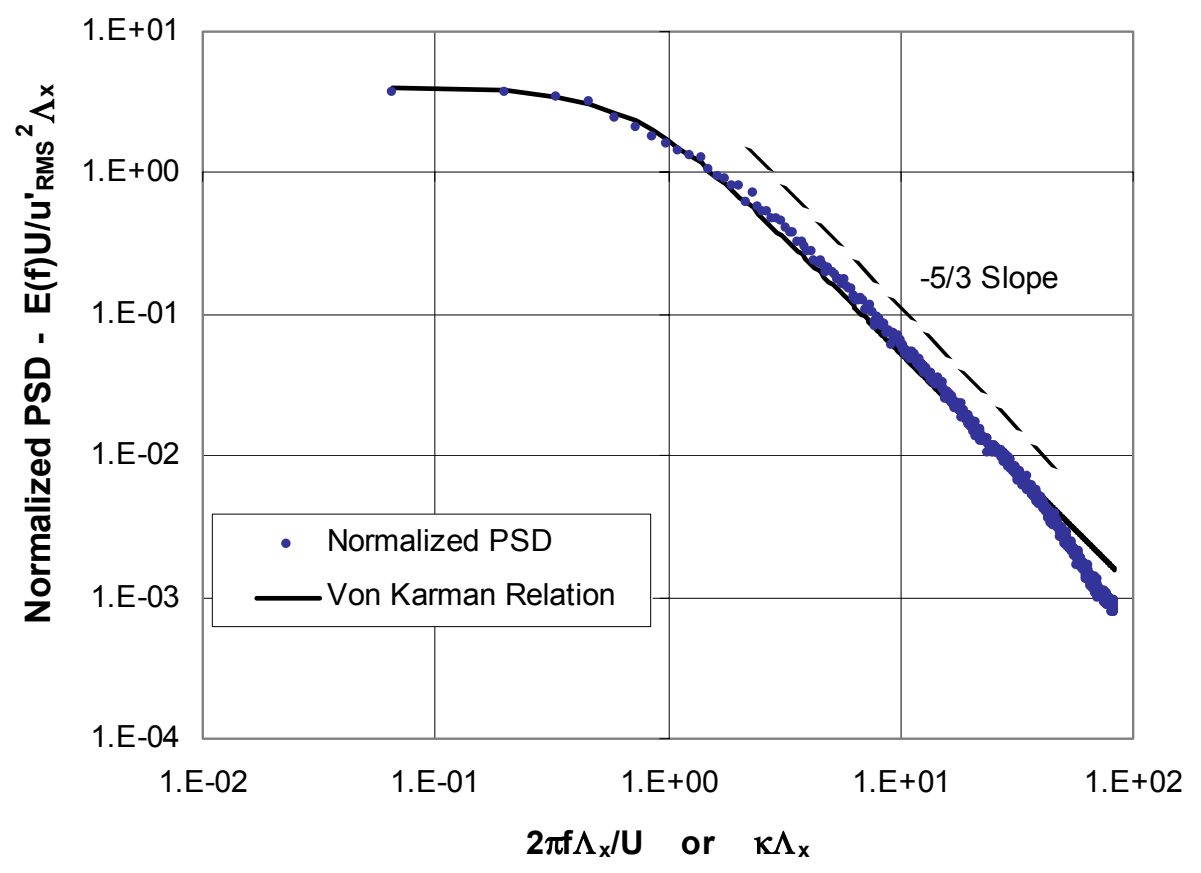

Figure 3.4: Normalized PSD and Von Kármán relation

\subsubsection{Coherence}

The correlation of two simultaneously sampled signals can be determined in the frequency domain through the coherence function, which is a measure of what frequencies are correlated between the two signals. Significant coherence at a given frequency indicates that the two signals have energy at the same frequency, which is correlated in time at that frequency. The coherence function is defined as the square of the cross-spectrum between the two signals divided by the product of the auto-spectrum of the two signals:

$$
\gamma_{x y}^{2}(f)=\frac{\left|S_{x y}\right|^{2}}{S_{x x} \cdot S_{y y}}
$$

By definition, the coherence function is equal to unity at all frequencies, however with a significant number of averages, the coherence function decreases from a value of unity at each frequency and asymptotically approaches a value representative of the correlation at each frequency. In essence, averaging of the coherence function eliminates uncorrelated content at each frequency such that only correlated content remains. For the current data, 
the time-resolved signals of velocity and heat flux (each with 131,070 data points) were broken up into smaller blocks (1024 data points) with 50\% overlap, such that approximately 256 averages were used to determine the coherence function.

\subsection{Steady Surface Heat Transfer}

This section presents the methodology for measuring steady surface heat transfer coefficients in the transonic wind tunnel, as well as results of steady measurements with and without high freestream turbulence. Surface heat transfer measurements were performed at three different locations on the blade surface, one on the suction surface (SS1) and two on the pressure surface (PS1 and PS2). The locations and flow conditions at these three locations are described in section 2.2.

\subsubsection{High Speed Flow Heat Transfer (edited from Nix, 1996)}

For low speed flows, the boundary layer energy equation in terms of enthalpy is:

$$
\rho \cdot\left(u \frac{\partial h}{\partial x}+v \frac{\partial h}{\partial y}\right)=k \frac{\partial^{2} T}{\partial y^{2}}
$$

For compressible, high-speed flows, the kinetic energy of the flow becomes important and the total enthalpy is used. Also, due to the speed of the flow, frictional heating can be significant and the viscous dissipation term must be considered. The energy equation in terms of total enthalpy for high speed flows then becomes:

$$
\left(u \frac{\partial h_{t}}{\partial x}+v \frac{\partial h_{t}}{\partial y}\right)=\alpha \frac{\partial^{2} h_{t}}{\partial y^{2}}+v \frac{\partial^{2}}{\partial y^{2}}\left(\frac{u^{2}}{2}\right)\left(1-\frac{1}{\operatorname{Pr}}\right)
$$

When $\operatorname{Pr}$ (Prandtl number)=1, the last term goes to zero and the energy equation is of the same form as the low speed energy equation (equation 3.13) and the heat transfer coefficient between the air and the blade is defined using the flow total temperature instead of the freestream static temperature: 


$$
q^{\prime \prime}=h \cdot\left(T_{t}-T_{w}\right)
$$

where $h$ is the convection heat transfer coefficient, $T_{t}$ is the total temperature of the flow, and $T_{w}$ is the surface (wall) temperature of the blades (measured at each gage location). When the Prandtl number is not equal to one $(\operatorname{Pr} \neq 1)$, which is the general case, then a proper temperature must be defined for the flow. The temperature typically used is the adiabatic wall or recovery temperature $\left(\mathrm{T}_{\mathrm{r}}\right)$. The recovery temperature is the temperature the wall would attain if $\mathrm{q}=0$. The heat transfer is then given by:

$$
q^{\prime \prime}=h \cdot\left(T_{r}-T_{w}\right)
$$

The recovery temperature $\left(T_{r}\right)$ is calculated using:

$$
T_{r}=T_{s}+r \cdot \frac{U_{\infty}{ }^{2}}{2 \cdot C_{p}}
$$

where $\mathrm{U}_{\infty}$ is the local freestream velocity of the flow, and $\mathrm{r}$ is the recovery factor. The recovery factor for laminar flows is approximated by $r=\sqrt{\operatorname{Pr}}$, while $r=\operatorname{Pr}^{1 / 3}$ for turbulent flow (Kays and Crawford, 1993). The recovery temperature can be calculated using the flow total temperature and the Mach number to find the freestream velocity, $\mathrm{U}_{\infty}$. Using the total temperature, the static temperature can be calculated using:

$$
T_{s}=\frac{T_{t}}{\left[1+\frac{\gamma-1}{2} M^{2}\right]}
$$

\subsubsection{Steady Heat Transfer Coefficient Methodology}

The methodology for determining heat transfer coefficients in the transient flows

of the transonic wind tunnel has been described by Popp, et al. (1999) and in Smith, et al. (2000). The following section outlines the methodology. 
It has been established that the driving force in convective heat transfer is the recovery temperature $\left(T_{r}\right)$ and Equation 3.16 governs heat transfer. Similar to Equation 3.17 for the recovery temperature, the total temperature of the flow is defined as:

$$
T_{t}=T_{s}+\frac{U_{\infty}^{2}}{2 \cdot C_{p}}
$$

The difference between the total temperature and the recovery temperature can be presented as:

$$
T_{t}-T_{r}=(1-r) \cdot \frac{U_{\infty}{ }^{2}}{2 \cdot C_{p}} \equiv T_{d}
$$

Rewriting equation 3.16:

$$
q^{\prime \prime}=h \cdot\left(T_{r}-T_{w}\right)=h \cdot\left(T_{t}-T_{w}\right)-h \cdot T_{d}
$$

Of the variables in this equation, the surface heat flux (q"), total temperature of the flow $\left(T_{t}\right)$ and the surface temperature $\left(T_{w}\right)$ are directly measured in the experiments. Due to the nature of the passive heat exchanger in the transonic wind tunnel, the total temperature of the flow drops throughout the course of an experiment and the wall temperature increases as heat is transferred from the mainstream flow. Figure 3.5a shows a sample time history of the relevant temperatures. 


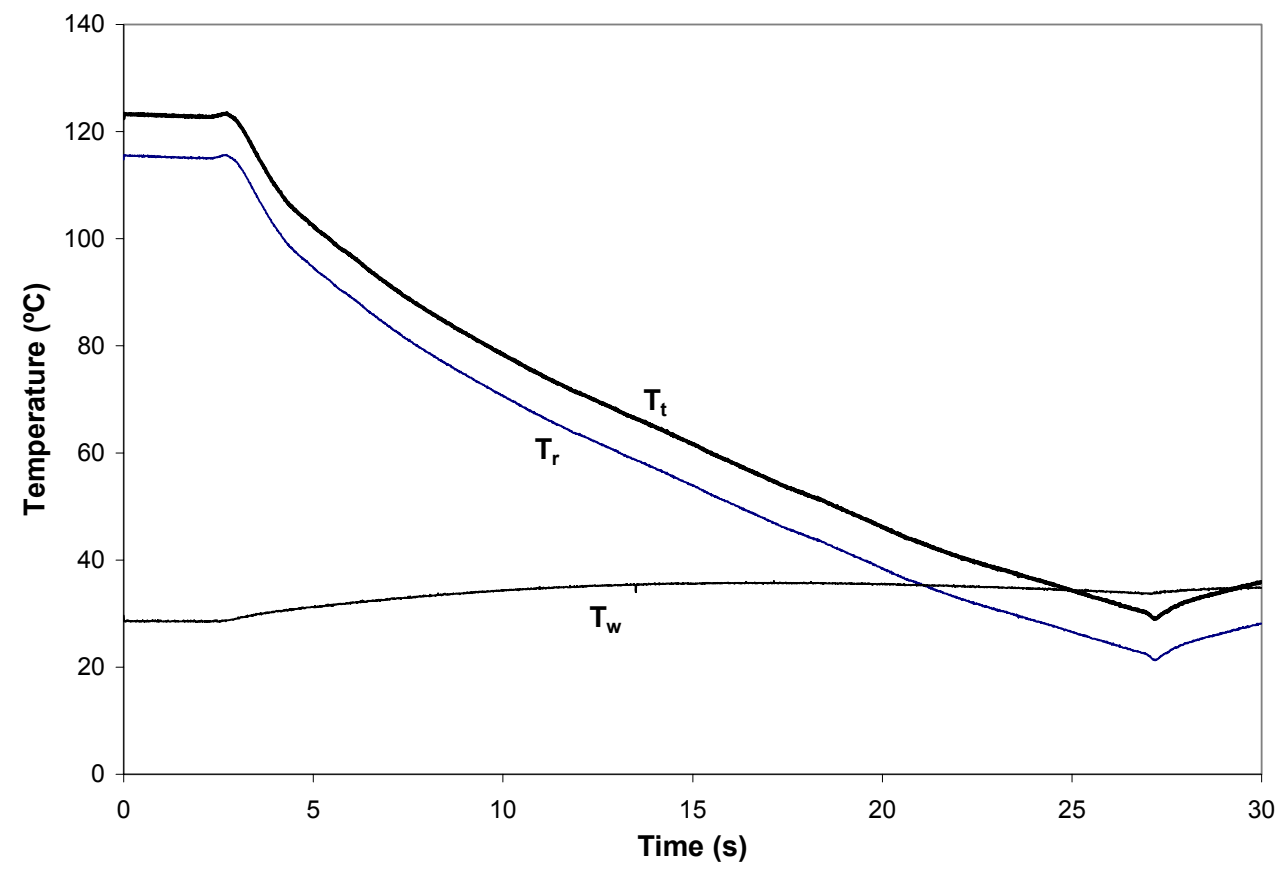

Figure 3.5a: Time history of relevant temperatures (SS1)

The dependent variable in Equation 3.21 (q") can be plotted against the independent variable $\left(\mathrm{T}_{\mathrm{t}}-\mathrm{T}_{\mathrm{w}}\right)$ and the slope of the resultant curve yields the heat transfer coefficient (h). The intercept of the equation, where $q=0$, results in $T_{d}=T_{t}-T_{w}$. Popp, et al. (1999) demonstrated that the heat transfer coefficient and $T_{d}$ did not significantly depend on the temperatures in Equation 3.21. These values are relatively constant during a tunnel run. Figure 3.5b shows a plot of the experimental data. Linear regression analysis is performed on the data to determine the slope and $\mathrm{x}$-axis intercept. With the value of $T_{d}$ known, the time history of the recovery temperature can be determined by Equation 3.20. 


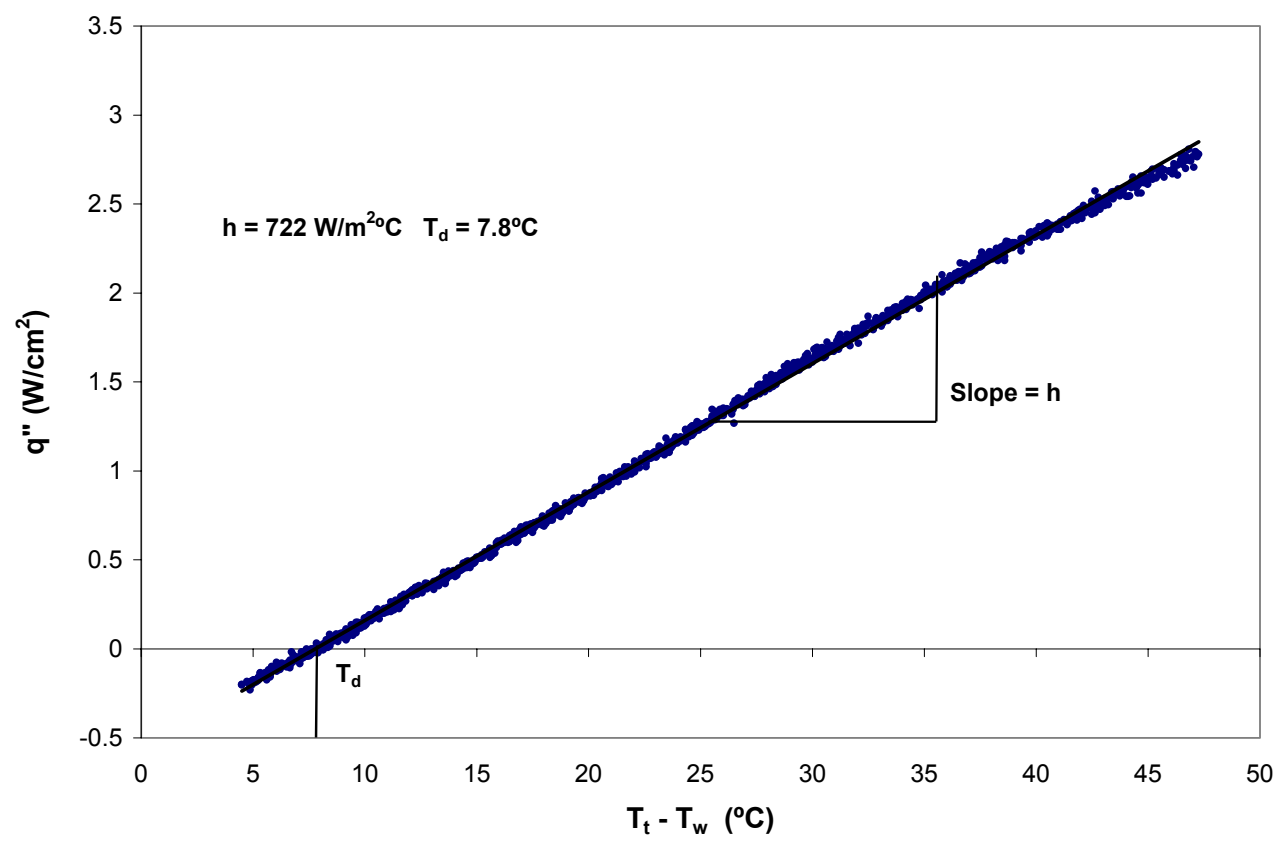

Figure 3.5b: Heat transfer data analysis (SS1)

For the purposes of the current analyses, the value of $T_{d}$ has little utility other than to allow the time history of the heat transfer coefficient to be plotted from the time histories of q", $\mathrm{T}_{\mathrm{t}}$, and $\mathrm{T}_{\mathrm{w}}$. Figure 3.6 plots the time history of the heat flux and the calculated heat transfer coefficient. As the recovery temperature and wall temperature converge (as shown in Figure 3.5a) the heat flux into the blade approaches zero. At this point the heat transfer coefficient calculations go unstable due to the driving temperature difference tending towards zero. 


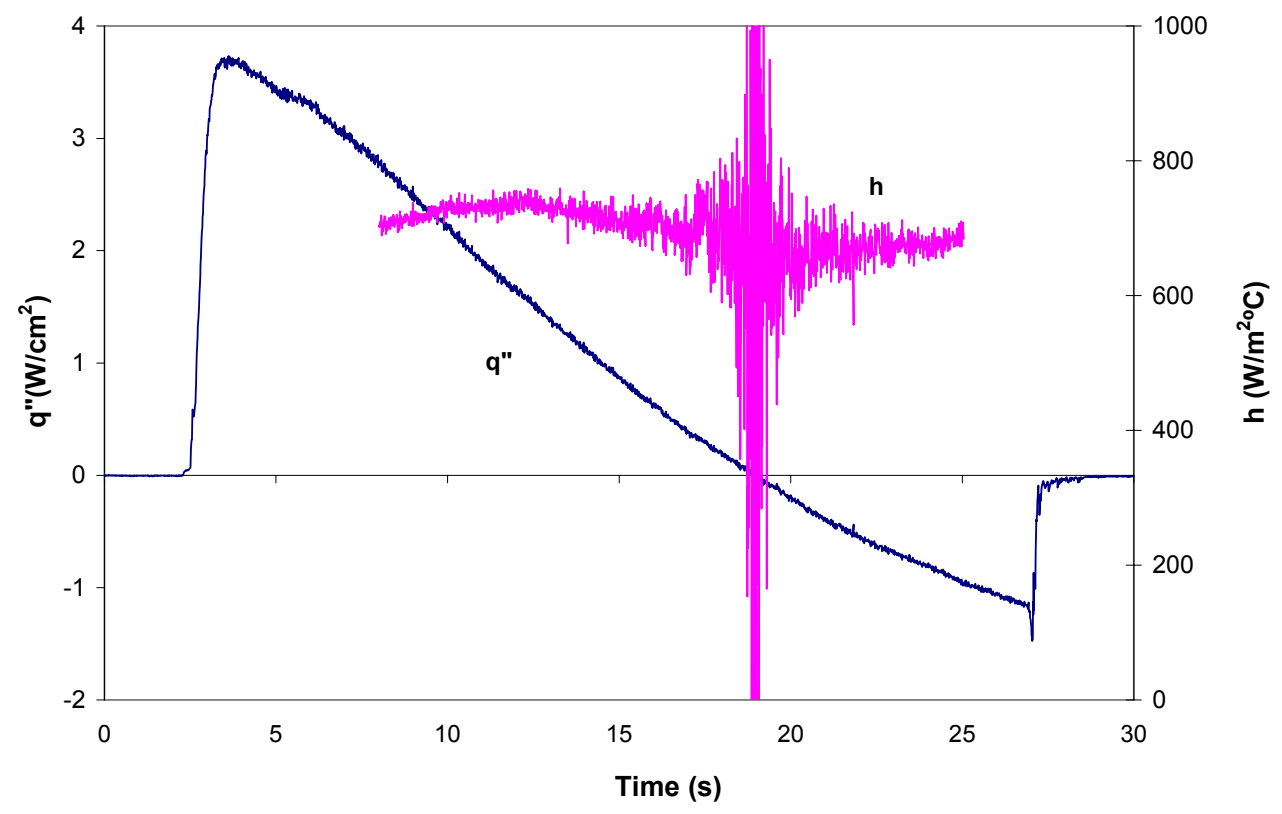

Figure 3.6: Time history of heat flux and heat transfer coefficient (SS1)

\subsubsection{Steady Heat Transfer Results}

Using the steady heat transfer analysis developed above, the heat transfer coefficients were determined from experimental measurements of the surface heat flux and relevant temperatures. As noted, the temperatures in the wind tunnel are transient, so the heat transfer is in essence a quasi-steady measurement. The flow and heat transfer in the wind tunnel never reach a steady state, however, the heat transfer coefficient is relatively steady throughout the tunnel run. The results for measurements with and without high freestream turbulence are discussed below.

\subsubsection{Low Turbulence Heat Transfer Coefficients}

As outlined in section 2.2, three locations on the instrumented turbine blade near the center of the cascade were instrumented with surface pressure, temperature and heat flux sensors. Two locations on the pressure surface and one location on the suction surface were instrumented. During a tunnel run, the necessary data were collected at each location in order to determine the heat transfer coefficients at each location. Several runs were performed over the course of several days to get a number of sample points to demonstrate repeatability and for uncertainty analyses. Table 3.1 summarizes the results 
of the heat transfer analyses with low turbulence along with measurement uncertainty. Background or "low turbulence" levels in the facility are less than $1 \%$.

The measured heat transfer coefficient data on the suction surface are in fairly good agreement with data reported by Smith, et al. (2002). The data on the pressure surface were compared with data collected on the pressure surface by Holmberg (1996). The data were considerably lower than reported in his work, where heat transfer coefficients in the range of $800-850 \mathrm{~W} / \mathrm{m}^{20} \mathrm{C}$ were reported. The data of Holmberg (1996) were reported to have large uncertainty in measurements and flow conditions were slightly different with the smaller turbine blades. The data collected in the current work are believed to be more accurate and are in better agreement with code predictions. In prior work in the transonic facility, calculations were performed by GE on the turbine blades using the KEP K-eps code modified from STAN-5. Details of the KEP code can be found in Zerkle (1988). Regarding the KEP model results, Holmberg (1996) mentions "it is important to note that heat transfer predictions with this code are tailored to match experience from full rig tests while the measurements here are made in a 2-D cascade." For comparison purposes, the data collected in this work are plotted versus the GE KEP code results in the next section (Figure 3.7), including results with high turbulence.

Table 3.1: Summary of heat transfer coefficients with low turbulence

\begin{tabular}{|c|c|c|c|}
\hline Run & \multicolumn{3}{|c|}{ Heat Transfer Coefficient $\left(\mathbf{W} / \mathbf{m}^{\mathbf{2}} \mathbf{C}\right)$} \\
\hline Number & SS1 & PS1 & PS2 \\
\hline $\mathbf{1}$ & 717 & 644 & 568 \\
\hline $\mathbf{2}$ & 703 & 623 & 551 \\
\hline $\mathbf{3}$ & 734 & 655 & 567 \\
\hline $\mathbf{4}$ & 725 & 651 & 567 \\
\hline $\mathbf{5}$ & 699 & 649 & 555 \\
\hline $\mathbf{6}$ & 736 & 691 & 588 \\
\hline Mean & 719 & 652 & 566 \\
\hline STDEV & 16 & 22 & 13 \\
\hline Uncertainty & $6.3 \%$ & $6.6 \%$ & $6.2 \%$ \\
\hline
\end{tabular}

\subsubsection{High Turbulence Heat Transfer Coefficients}

Measurements of steady heat transfer with high turbulence were performed and compared with the low turbulence measurements and code predictions. The turbulence 
grid was installed and identical procedures were used to acquire the data. The upstream turbulence field approaching the cascade blade passages was characterized with a streamwise traverse of a hot-wire probe (see Appendix A) in order to determine the turbulence conditions near the entrance to the cascade passages. The results indicated a turbulence intensity of approximately $10 \%$ with a length scale of $2.0 \mathrm{~cm}$ near the entrance to the cascade passages (see plot on pg. 130 of Appendix A). These conditions are heretofore referred to as "high turbulence". The results for high turbulence experiments sampled at each of the three blade measurement locations are presented in Table 3.2 along with measurement uncertainty and compared with the low turbulence results. Several runs were performed to demonstrate repeatability and for uncertainty analysis. The uncertainty in the increase in heat transfer coefficient due to turbulence is only a function of precision uncertainty (from repeatability) since the bias uncertainty of the heat flux gages is present in both measurements. The uncertainty in the measured increase is shown in Table 3.2 and is an average of $2.9 \%$ (much lower than the measure percentage increase).

Table 3.2: Summary of heat transfer coefficients with high turbulence

\begin{tabular}{|c|c|c|c|}
\hline Run & \multicolumn{3}{|c|}{ Heat Transfer Coefficients $\left(\mathbf{W} / \mathbf{m}^{\mathbf{2}} \mathbf{C}\right)$} \\
\hline Number & SS1 & PS1 & PS2 \\
\hline $\mathbf{1}$ & 795 & 776 & 671 \\
\hline $\mathbf{2}$ & 788 & 768 & 668 \\
\hline $\mathbf{3}$ & 771 & 761 & 657 \\
\hline $\mathbf{4}$ & 762 & 750 & 650 \\
\hline Mean & 779 & 764 & 662 \\
\hline STDEV & 15 & 11 & 10 \\
\hline Uncertainty & $6.4 \%$ & $6.2 \%$ & $6.2 \%$ \\
\hline Low Turbulence HTC & 719 & 652 & 566 \\
\hline \% Increase over Low Tu & $8.3 \%$ & $17.1 \%$ & $16.9 \%$ \\
\hline Uncertainty in Increase in h & $2.9 \%$ & $3.2 \%$ & $2.5 \%$ \\
\hline
\end{tabular}

The results shown in Table 3.2 indicate that there is a more significant increase in heat transfer coefficient on the pressure surface due to high turbulence as compared to the suction surface. The increase in heat transfer on the pressure surface $(17 \%)$ is approximately two times greater than that measured on the suction surface $(8 \%)$. This 
corresponds to increases in blade surface temperature of $4-10 \%$ (between 50 and $100^{\circ} \mathrm{C}$ ). Increases in temperature of $25^{\circ} \mathrm{C}$ or more can significantly decrease the life of turbine materials. This result is in good agreement with work by previous researchers on both turbine blades and vanes (see section 1.2.1.3). The increase in heat transfer on the pressure surface gages is similar in terms of percentage increase, but the absolute increase in heat transfer coefficient is larger at location PS1 (closer to the leading edge) than at location PS2. As noted earlier, the two gages are located in regions of relatively similar velocity; however, the flow at PS1 experiences a slightly adverse pressure gradient while location PS2 sees a favorable pressure gradient.

The results with low and high turbulence were compared with GE KEP code predictions for the turbine blade and with data from Holmberg (1996) and Smith, et al. (2000). The results are presented in Figure 3.7 with measurement uncertainty error bars. As shown in the figure, the code predicts a much higher increase in heat transfer due to turbulence on the pressure surface as compared with the suction surface. This result is consistent with the current data, although the current data with high turbulence agrees better with the code predictions at $5 \%$ inlet turbulence. It should be noted that the heat transfer predictions with the KEP code were tailored to match experience from full-rig tests, while measurements here are in a linear 2-D cascade (Holmberg, 1996). At present, the KEP code data are the only numerical results available for this blade geometry for comparison with experimental measurements. The data for the suction surface location (SS1) without turbulence agree well with the data from Smith, et al. (2000). The data from Holmberg (1996) on the pressure surface are much higher than the current data. Holmberg reported very high uncertainty in his measured data, as high as $20 \%$ uncertainty (shown in figure). 


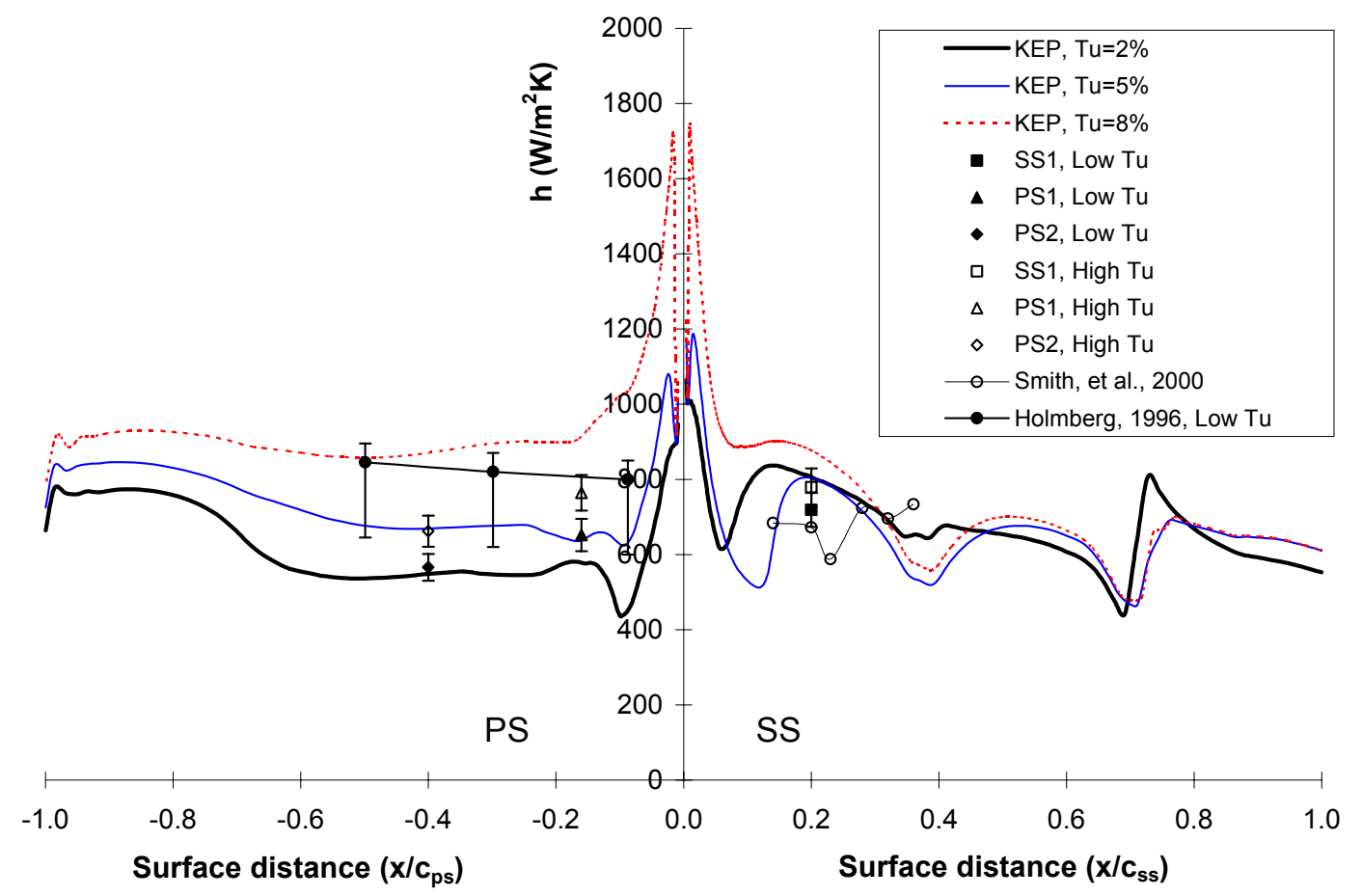

Figure 3.7: Heat transfer coefficient data compared with KEP code predictions and previous data

\subsection{Time-Resolved Velocity and Heat Transfer}

Time-averaged (steady) heat transfer results for the current work with and without high turbulence have been presented in the previous sections. In addition to measuring the effects of turbulence on the mean heat transfer, the goal of this work was to investigate time-resolved surface heat flux and the interaction of unsteady velocity of the mainstream flow near the blade surface with surface heat flux. Analysis of the timeresolved signals may provide insight into the mechanism by which freestream turbulence affects surface heat transfer.

As discussed earlier, measurements of time-resolved velocity were performed with a hot-wire probe (PHW) located approximately $5 \mathrm{~mm}$ above the surface of the three instrumented locations of the turbine blade (see Figure 2.8). This distance is outside of the boundary layer and far enough from the blade surface to insure the probe will not impact the blade surface (and destroy the wire) if severe vibration occurred or during startup and shutdown of the wind tunnel. The boundary layer thickness was estimated based on measurements by Wesner (1996) and scaling of analyses by Holmberg (1996) to be approximately $0.3 \mathrm{~mm}$ over most of the suction surface and between 0.3 and $1.5 \mathrm{~mm}$ 
on the pressure surface. Data from Radomsky (1999) and Ames (1994) demonstrated that levels of velocity fluctuations outside the boundary layer but near the vane surface are relatively constant. The $5 \mathrm{~mm}$ distance is outside of the boundary layer, but close enough to measure mean and fluctuating velocities near the edge of the boundary layer.

Figure 3.8 shows an illustration of the hot-wire setup. The heat flux gages at these instrumented locations are capable of high frequency measurements to obtain a time-resolved signal of surface heat transfer. The following sections discuss the comparisons of the signals through analysis in both the time domain and the frequency domain. These analyses are limited, but some key observations are made about how the freestream velocity fluctuations interact with surface heat transfer.

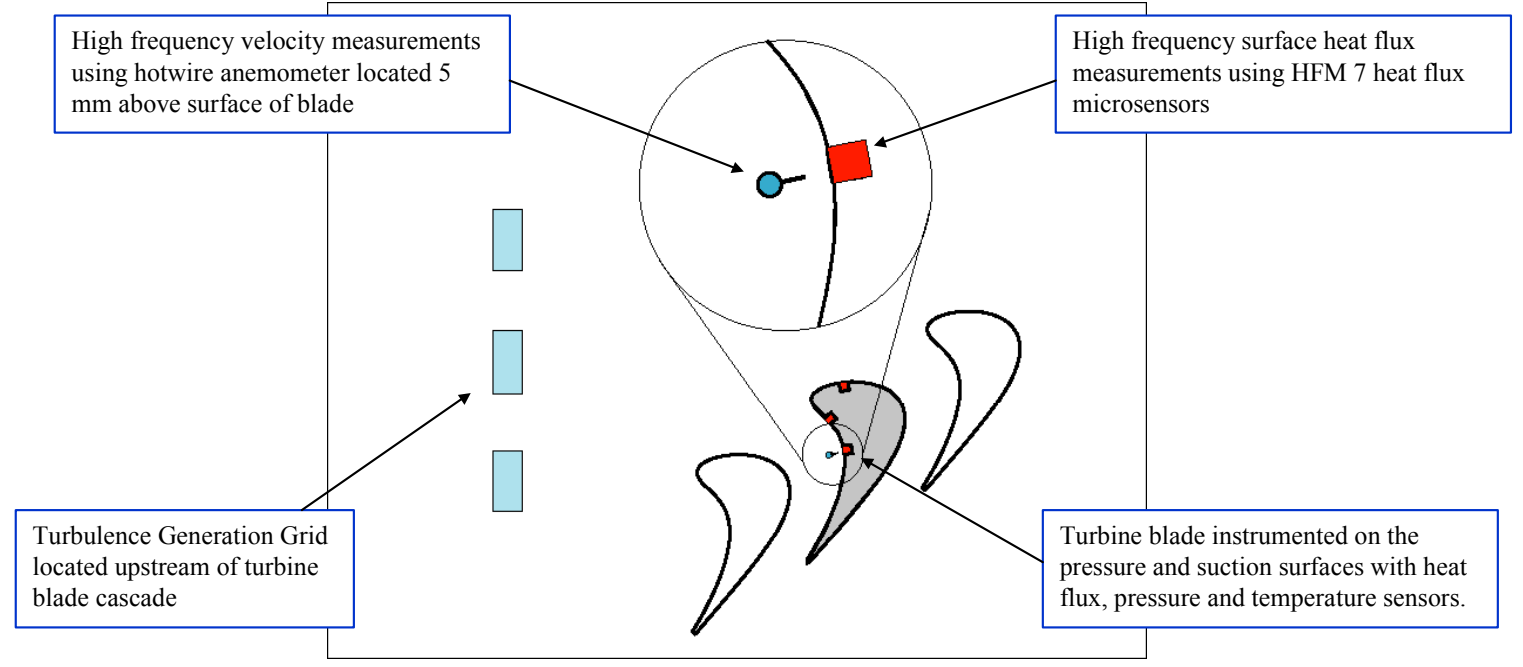

Figure 3.8: Illustration of simultaneous velocity and heat flux measurements

\subsubsection{Time Domain Analysis}

\subsubsection{Direct Time Signal Comparison}

Comparisons of the time-resolved velocity and heat flux signals were performed in the time-domain to investigate the correlation between the two signals. Figure 3.9a Figure 3.9c show a section of the time-resolved velocity and heat flux signals normalized by their mean values for all three measurements locations. The traces of velocity and heat flux demonstrate that there is low frequency correlation between the two signals on the pressure surface (PS1 and PS2), as peaks in velocity show corresponding peaks in heat flux. The low frequency energy of the velocity signal appears to be in phase with 
the corresponding energy in the heat flux signal. It is apparent from the figures that higher frequency fluctuations in the signals do not appear to be correlated. These results are similar to data reported in work by Moss and Oldfield (1994). From the plot for the suction surface gage (SS1) it can be seen that the fluctuations in velocity are much smaller when normalized by the mean velocity (low turbulence intensity) and that there does not appear to be a strong correlation between the fluctuations in heat flux with fluctuations in velocity. The signals for all three locations in Figure 3.9 are plotted on the same scale for direct comparison between locations.

Comparisons of the velocity and heat flux signals in this fashion are a weak analysis technique except when there is a significant level of fluctuation in both signals (as is the case with the pressure surface locations). Frequency domain correlation is a much stronger tool for direct comparison of two simultaneously sampled signals, which will be presented later. Frequency domain analysis allows for correlation of the energy across the spectrum of frequencies as well as phase information.

Using the analysis tools presented in Equations 3.3 - 3.5, the turbulence intensity and heat flux intensity of the signals at each gage location were determined and are shown in Table 3.3. The turbulence intensity is normalized by the local mean velocity. Interestingly, the intensity of turbulent fluctuations (independent of normalization by the local mean velocity) is shown to be very low on the suction surface and much higher on the pressure surface. The turbulent fluctuations on the pressure surface are shown to decrease slightly as the flow progresses from PS1 to PS2.

Measurements of cross-stream (or pitch-wise) variations of turbulence intensity downstream of the grid (presented in Appendix A) showed little variation in intensity so differences in suction surface versus pressure surface turbulence is not due to nonuniformity of inlet turbulence. The higher intensity of fluctuations on the pressure side versus the suction surface qualitatively agrees with the data from Holmberg (1996), although the magnitude of fluctuations and relative difference between the two surfaces is much higher in this case. However, as pointed out earlier, the approaching turbulence intensity and scale are much higher in this work. The low speed turbine vane turbulence data from Radomsky and Thole (1998) also demonstrated that the turbulence levels on the pressure surface were higher than those measured on the suction surface. 
Table 3.3: Turbulence and heat flux intensity

\begin{tabular}{|c|c|c|c|c|}
\hline Gage Location & $\mathbf{u}^{\prime}{ }^{\prime}$ ms $(\mathrm{m} / \mathbf{s})$ & Tu $(\%)$ & $\mathbf{q}^{\prime}{ }^{\prime}{ }^{\prime}\left(\mathbf{W} / \mathbf{c m}^{2}\right)$ & $T u_{\mathbf{q}}(\%)$ \\
\hline SS1 & 9.31 & 4.5 & 0.39 & 15.9 \\
\hline PS1 & 20.97 & 18.8 & 0.28 & 18.6 \\
\hline PS2 & 17.47 & 12.5 & 0.38 & 18.1 \\
\hline
\end{tabular}

From the table, there does not appear to be any correlation between turbulence intensity $(\mathrm{Tu})$ and heat flux intensity $\left(\mathrm{Tu}_{\mathrm{q}}\right)$. This is consistent with results reported by Holmberg (1996). The two pressure surface locations have very similar values of $\mathrm{Tu}_{\mathrm{q}}$, however there is a fairly significant difference in turbulence intensity between the two locations. The suction surface location shows a large value of $\mathrm{Tu}_{\mathrm{q}}$, however the turbulence intensity at this location is very low as is apparent by observation of Figure 3.9a. Through comparison of the absolute values of $\mathrm{u}_{\mathrm{rms}}^{\prime}$ and $\mathrm{q}_{\mathrm{rms}}^{\prime}$ (rather than the values of $\mathrm{Tu}$ and $\mathrm{Tu}_{\mathrm{q}}$ which are normalized by local measured mean velocity and heat flux) there still does not appear to be good correlation.

If the value of $\mathrm{u}_{\mathrm{rms}}^{\prime}$ is compared to the value of $T \mathrm{u}_{\mathrm{q}}$, there seems to be a fairly linear relationship. In these tests, the mainstream flow was not heated, rather the driving temperature difference for heat transfer was achieved by cooling the blade surface prior to running the tests. Using unheated mainstream air reduced uncertainties that may exist in whether fluctuations in surface heat flux were due to fluctuations in heat transfer coefficient, or due to fluctuations in temperature. In addition, unheated mainstream flow reduces uncertainty in hotwire calibration and conversion of hotwire voltage into velocity data. Since there is little variation in the mainstream temperature and the blade surface temperature does not change significantly in the short sampling time, the quantity $\mathrm{Tu}_{\mathrm{q}}$ is essentially equal to $h_{r m s}^{\prime} / \bar{h}$. Since there appears to be a correlation between $u_{r m s}^{\prime}$ and $T u_{q}$, it would stand to reason that $\mathrm{u}_{\mathrm{rms}}^{\prime}$ would be valuable in predicting increases in heat transfer. This has been demonstrated in many of the correlations on flat plates and cylinders and will be used later in this work in the development of an analytical model to predict increases in heat transfer due to turbulence. 


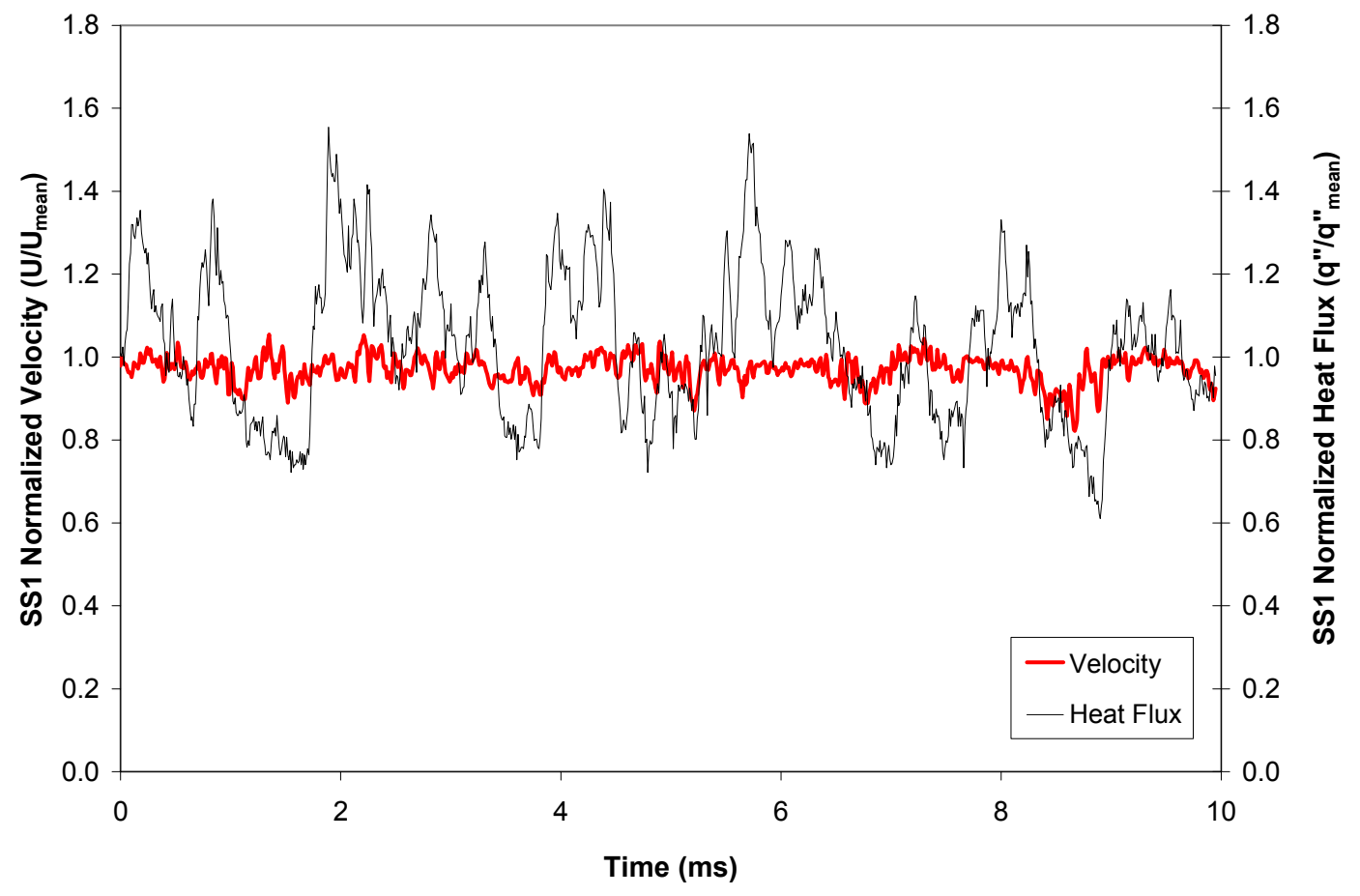

a. Location SS1

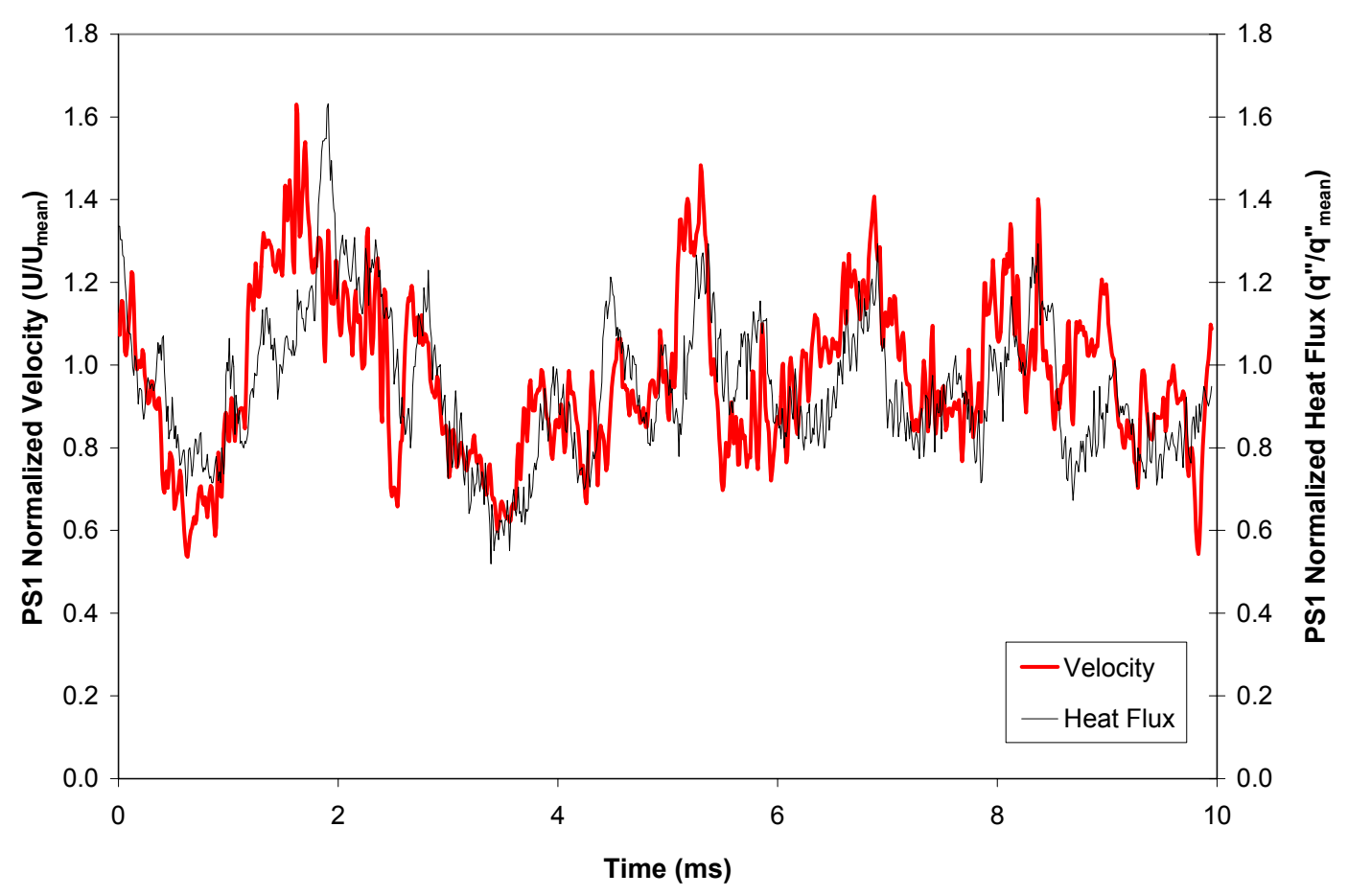

b. Location PS1 


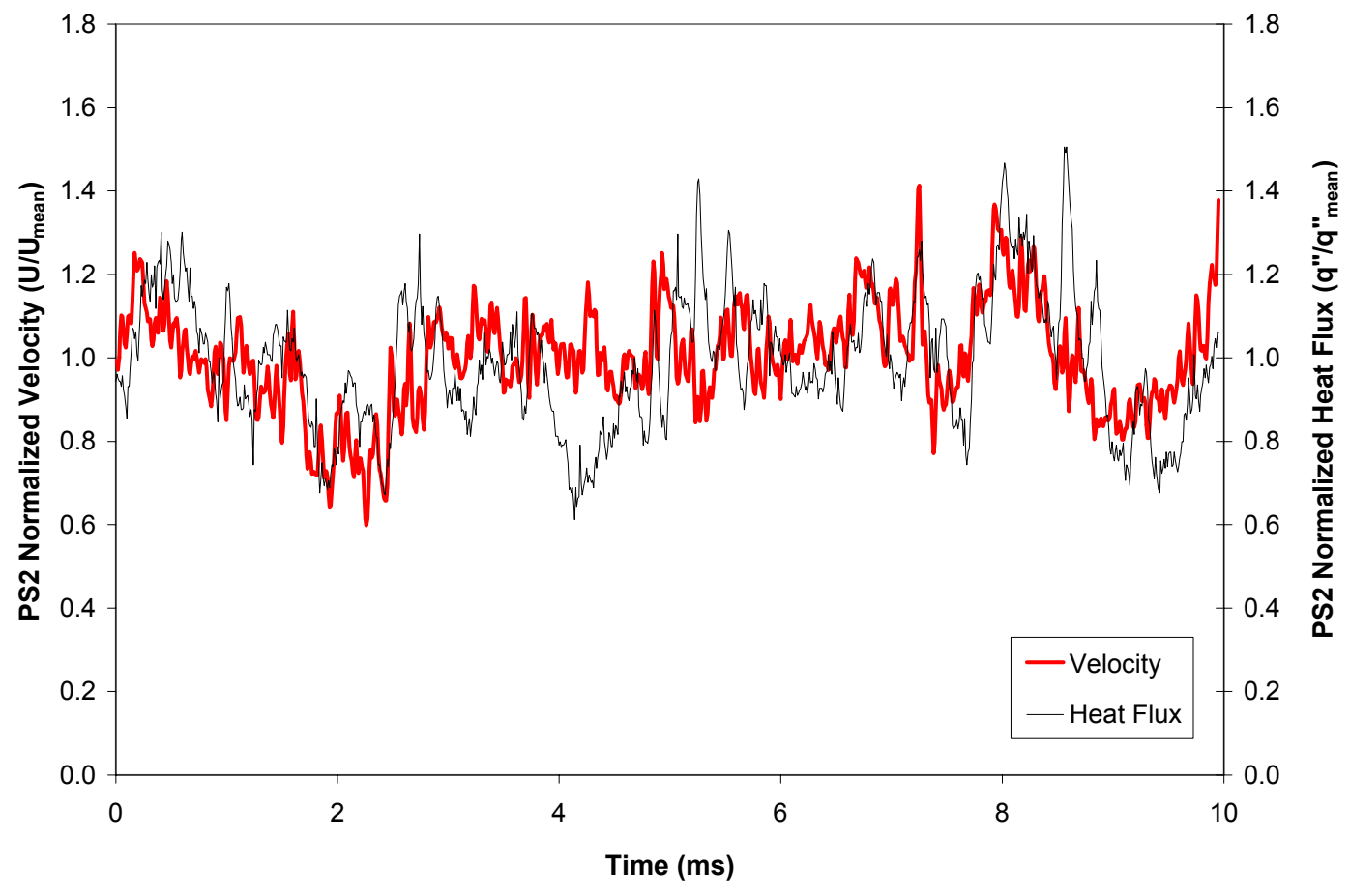

c. Location PS2

Figure 3.9: Time-resolved velocity and heat flux signals

\subsubsection{Time Correlation and Scales}

The autocorrelation function for both the velocity and heat flux signals was calculated to determine the time scale of the fluctuations in each. Figure 3.10a-Figure $3.10 \mathrm{c}$ present the autocorrelation results for each gage location. As shown in the figures, the measured velocity and surface heat flux have very similar time scales at the two pressure surface locations (PS1 and PS2). The suction surface location (SS1) demonstrates a smaller time scale for the heat flux signal as compared to the velocity signal. The results of calculated velocity and heat flux time scales and streamwise integral length scale of velocity are presented in Table 3.4.

Table 3.4: Time scales and integral length scales

\begin{tabular}{|c|c|c|c|}
\hline Gage Location & $\mathrm{T}_{\mathrm{u}}(\mathbf{m s})$ & $\Lambda_{\mathrm{x}}(\mathbf{c m})$ & $\mathrm{T}_{\mathbf{q}}(\mathbf{m s})$ \\
\hline SS1 & 0.18 & 3.46 & 0.14 \\
\hline PS1 & 0.21 & 2.39 & 0.20 \\
\hline PS2 & 0.18 & 2.51 & 0.18 \\
\hline
\end{tabular}


As the table shows, the integral length scale calculated on the suction surface is larger than the pressure surface locations. The approaching length scale is approximately $2 \mathrm{~cm}$ and increases slightly as the flow proceeds along the two pressure surface locations. There is a fairly large uncertainty in determination of the integral length scale (as will be presented in Appendix B), but the scale determined for the suction surface location is considerably larger than the measured upstream and pressure surface length scales. The time scales are relatively similar, but due to the high velocity in the suction surface, the calculated length scale is higher. Measurements on the suction surface have demonstrated smaller turbulence fluctuations and larger scale than the measurements on the pressure surface.

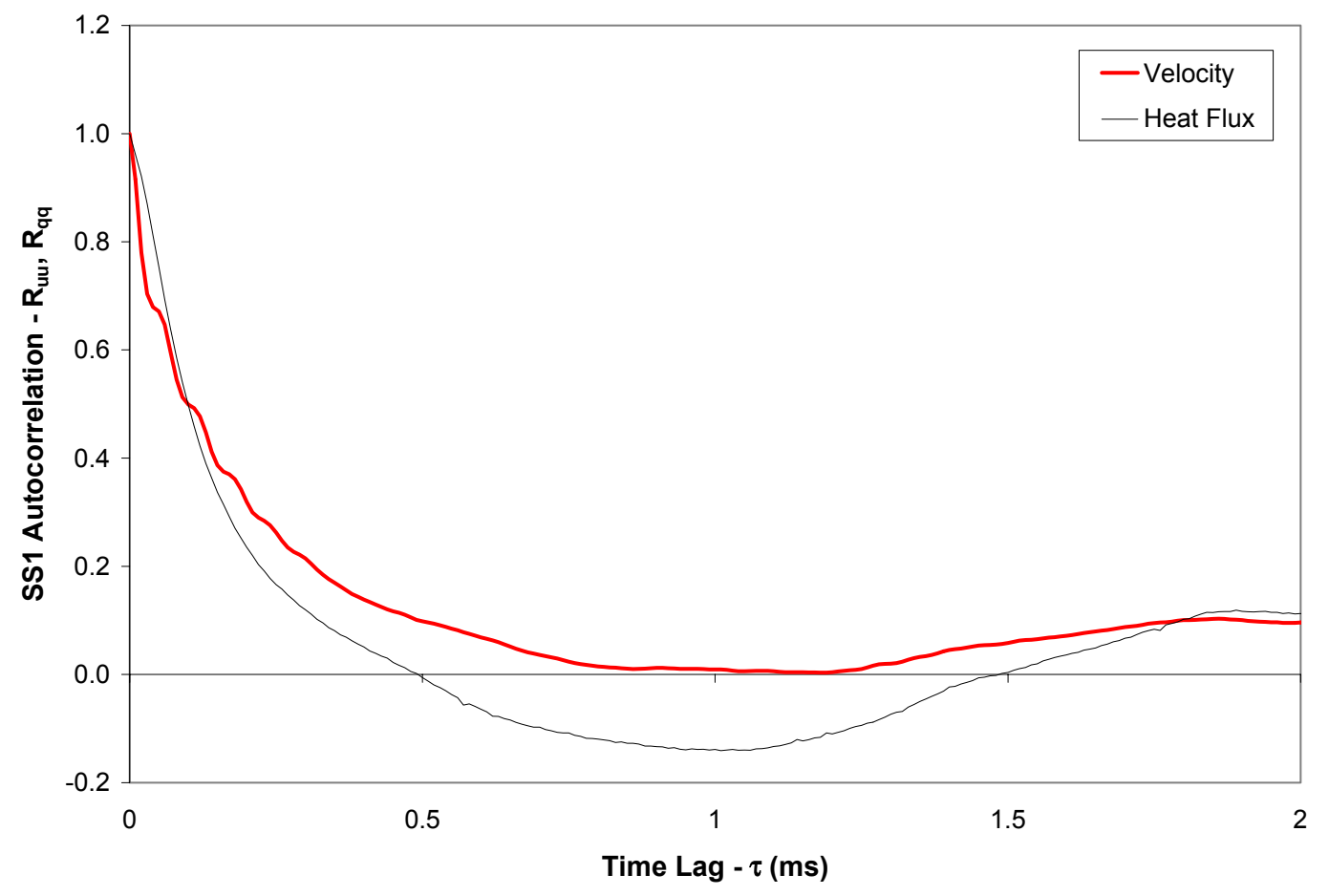

a. Location SS1 


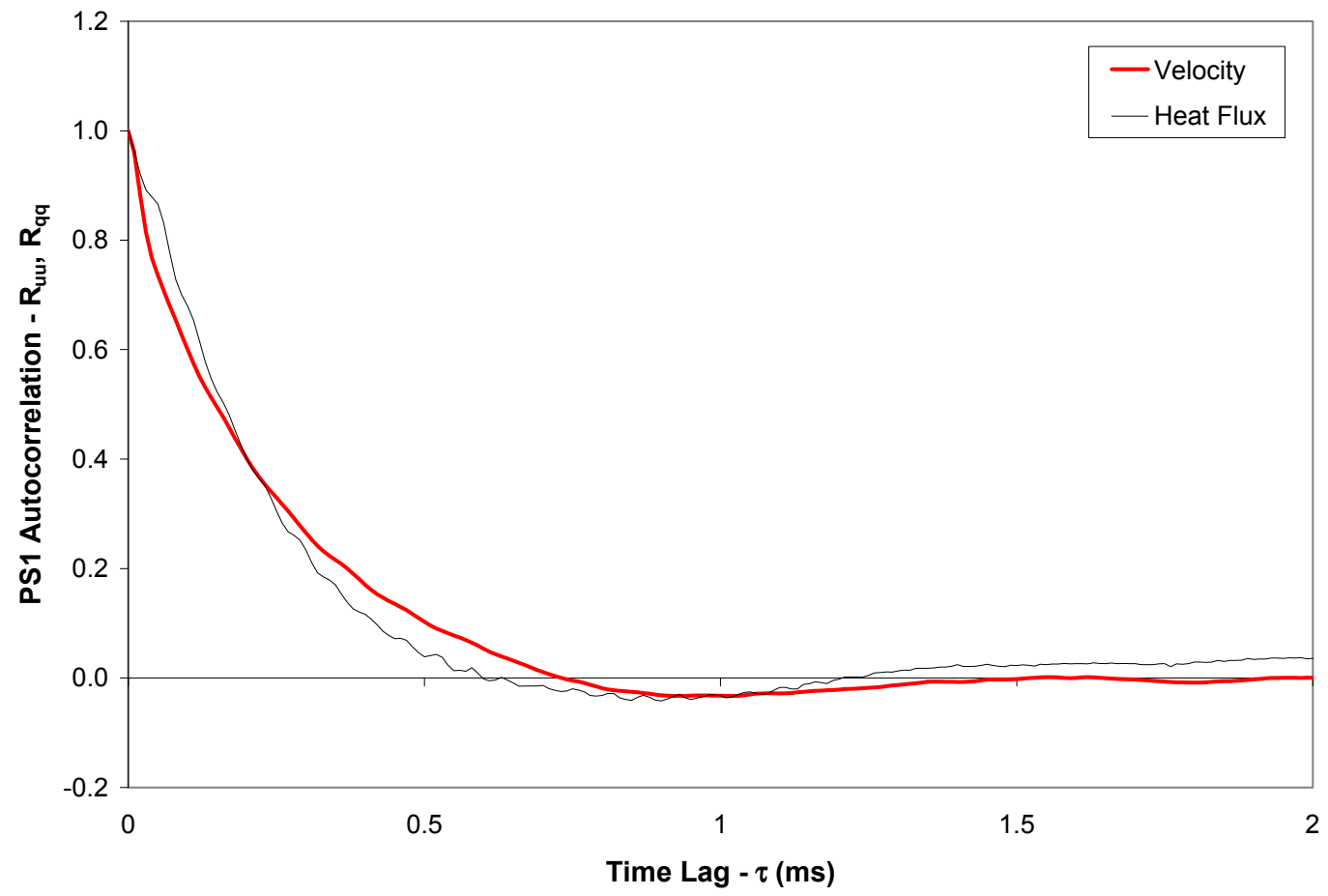

b. Location PS1

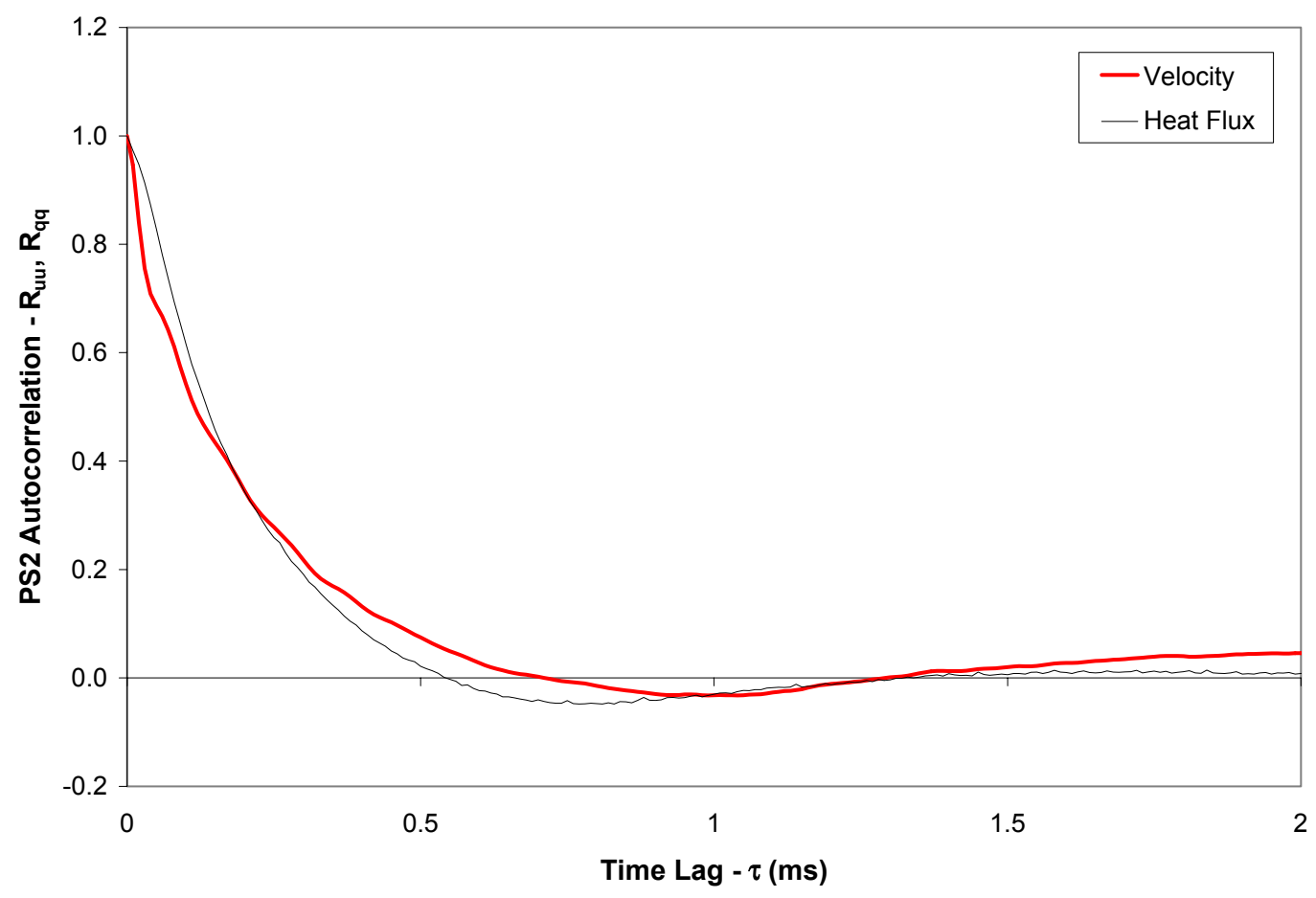

c. Location PS2

Figure 3.10: Autocorrelation of velocity and heat flux signals 


\subsubsection{Frequency Domain Analysis}

\subsubsection{Velocity and Heat Flux Spectra}

The time resolved measurements of velocity and heat flux were converted into the frequency domain to determine the velocity frequency spectra above the heat flux gages and the heat flux spectra at each location. Figure $3.11 \mathrm{a}-3.11 \mathrm{c}$ show the velocity and heat flux spectra for each gage location. Figure 3.12 shows a comparison of the unnormalized velocity spectra for all three measurement locations for comparison of the energy at each location, while Figure 3.13 shows a comparison of the heat flux spectra at all three measurement locations. Figure 3.14 shows the velocity spectra from the three locations normalized and plotted against the theoretical Von Kármán relation for onedimensional spectra.

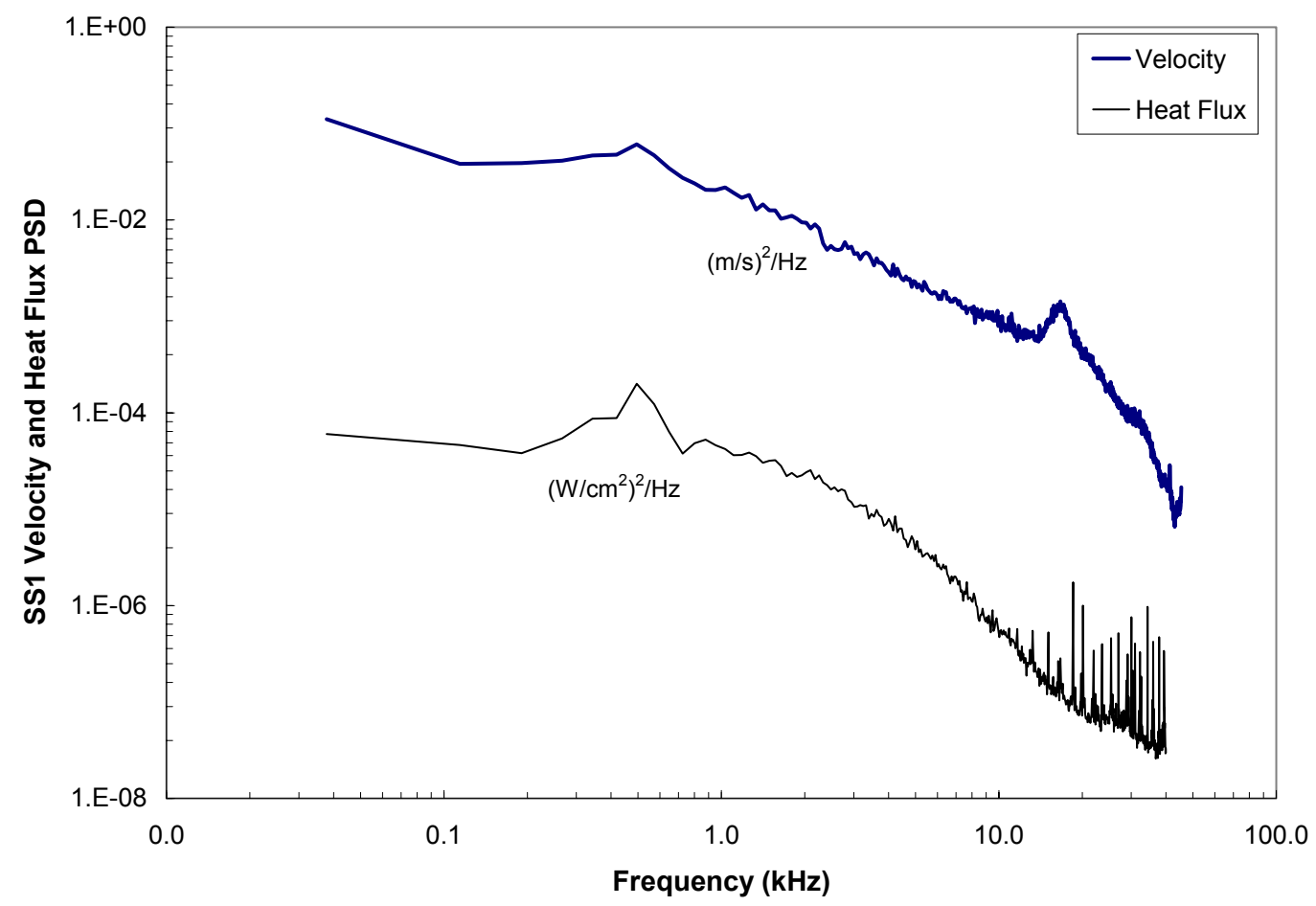

a. Location SS1 


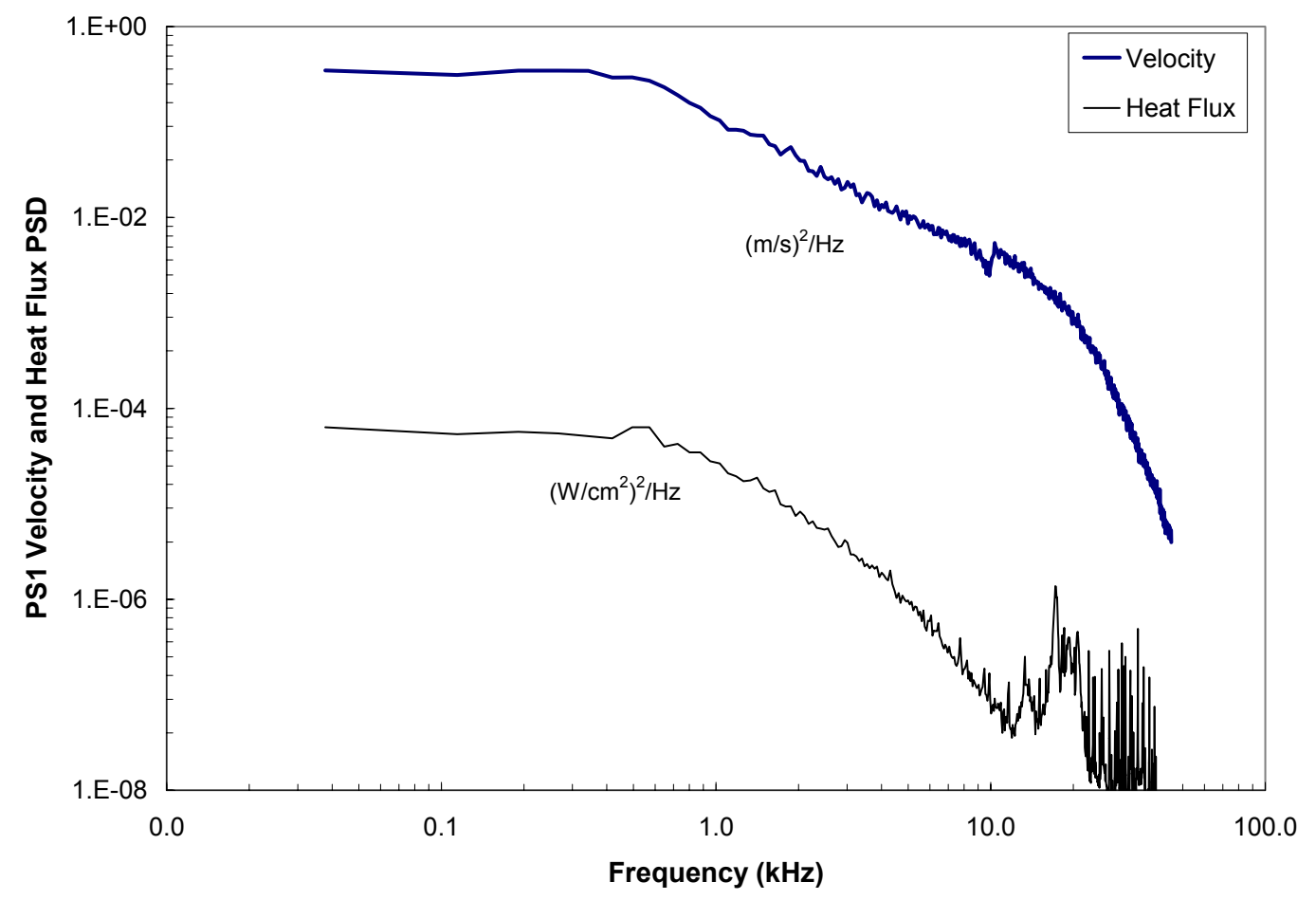

b. Location PS1

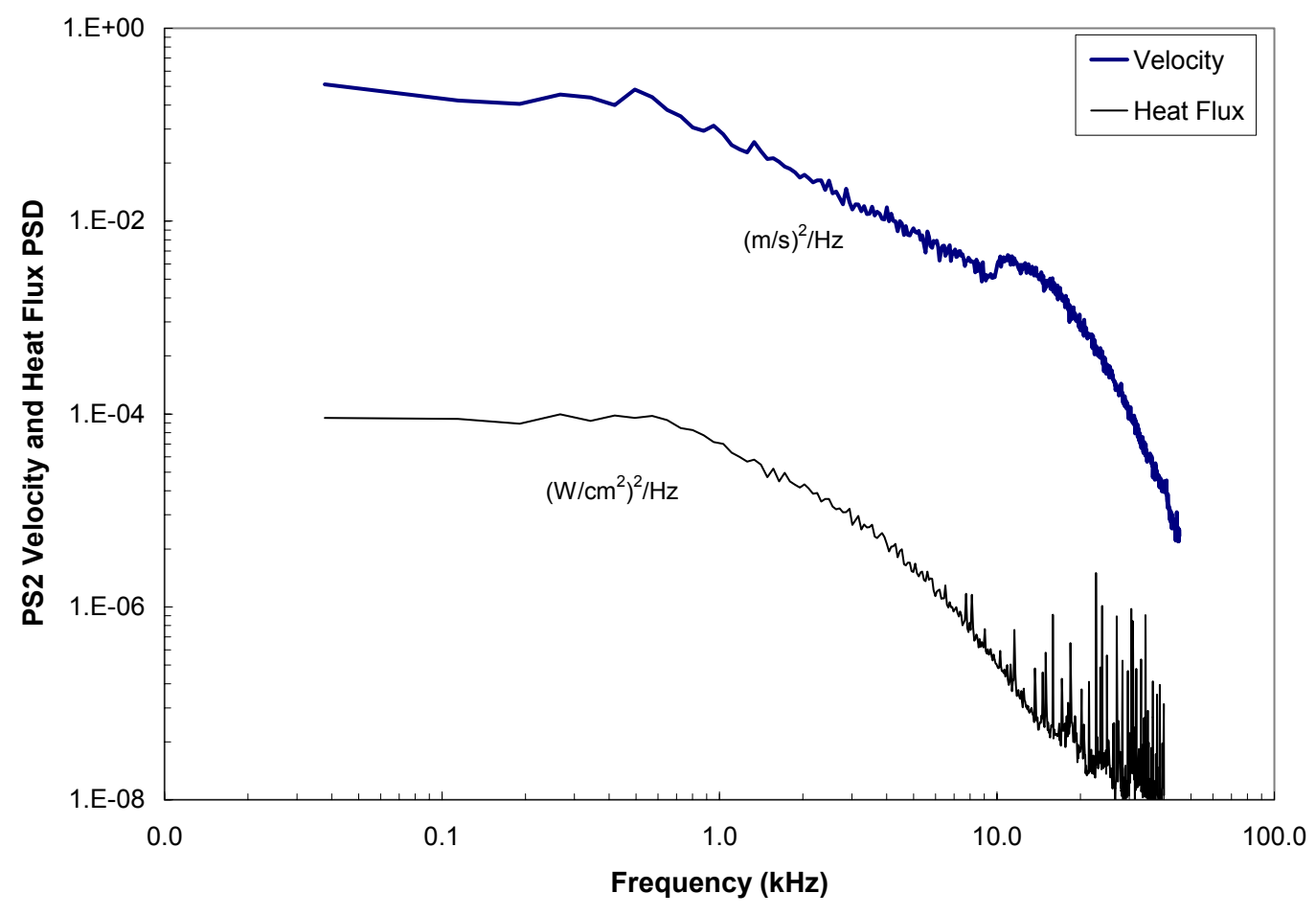

c. Location PS2

Figure 3.11: Velocity and heat flux spectra 


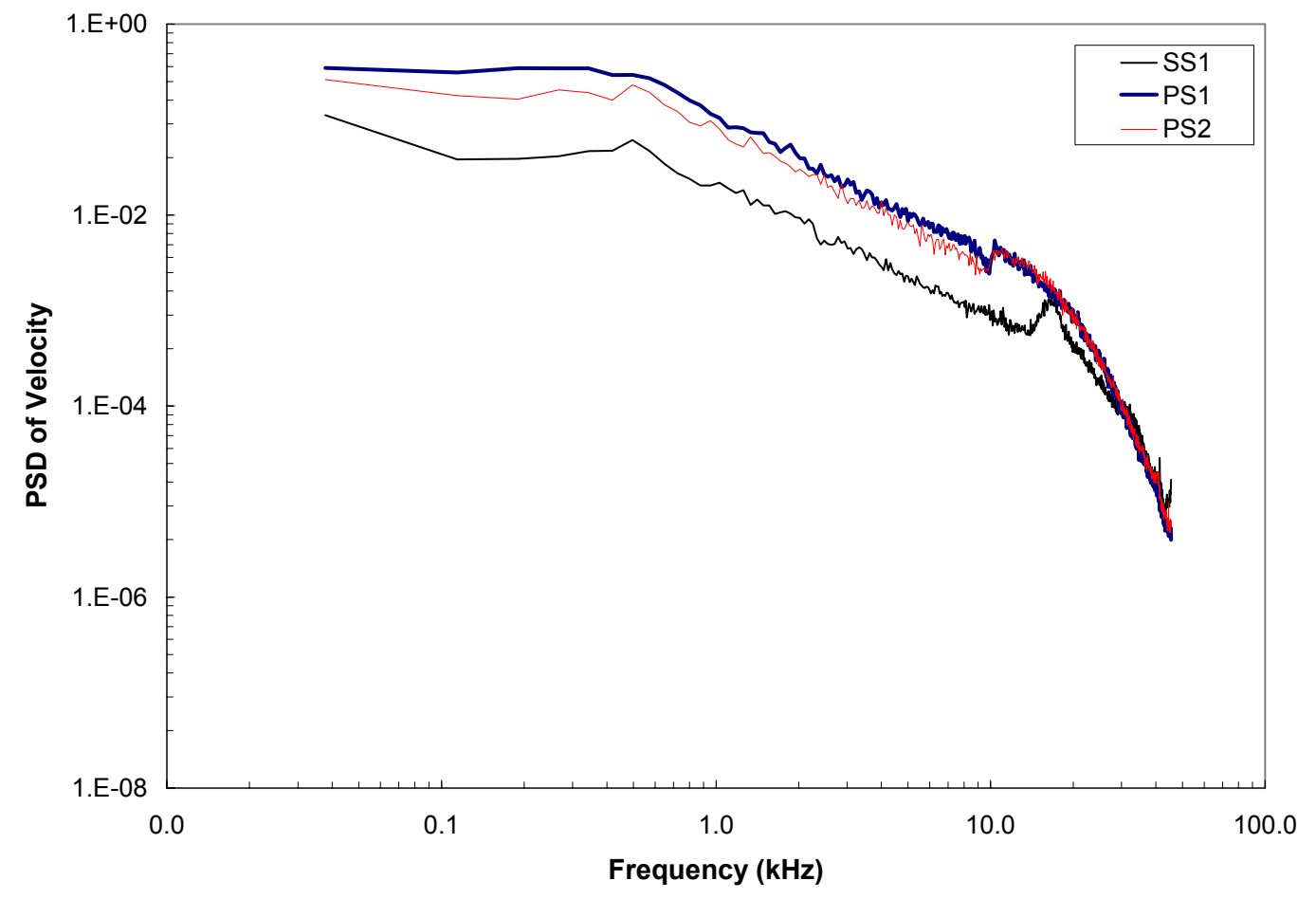

Figure 3.12: Comparison of velocity spectra at all measurement locations

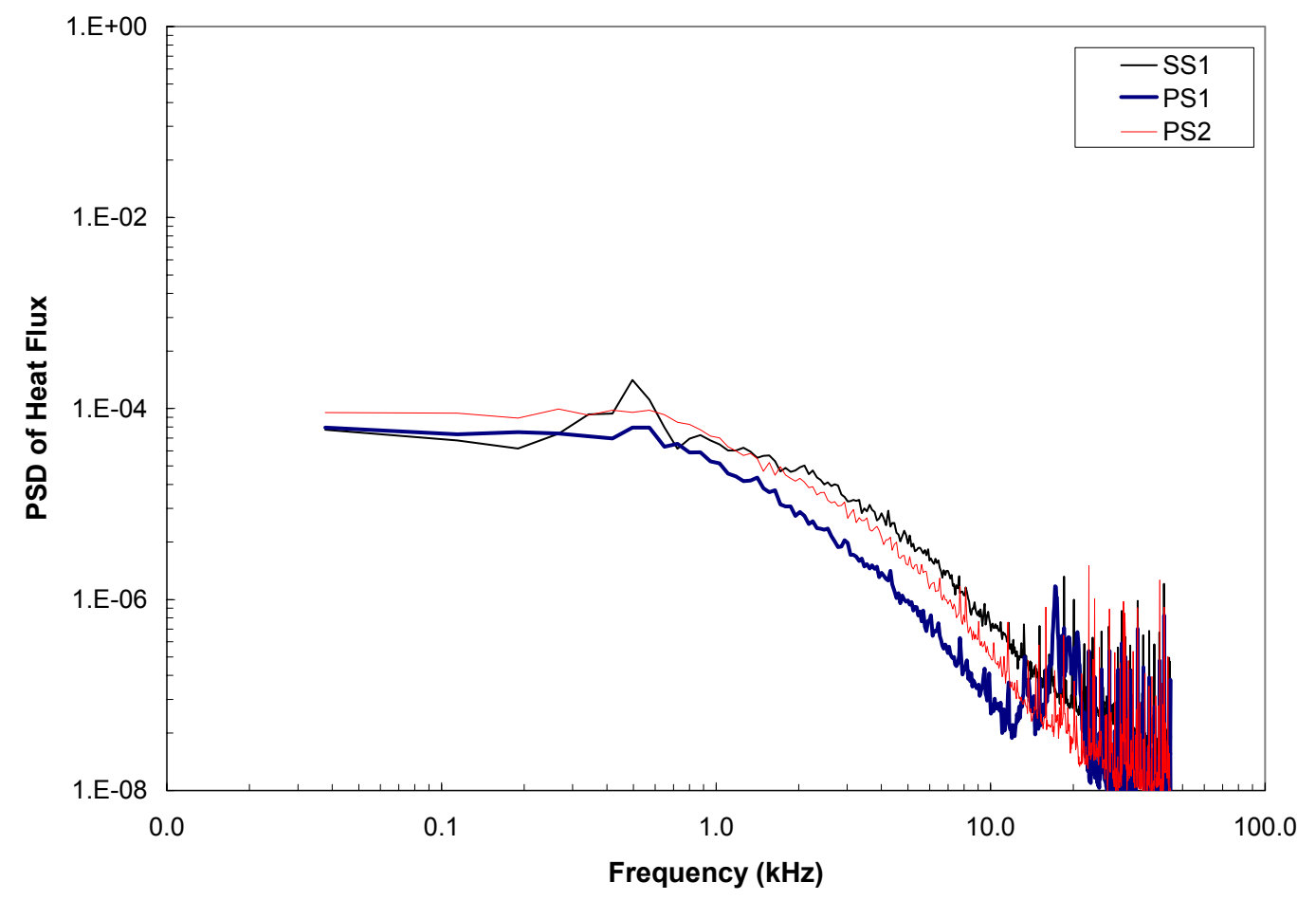

Figure 3.13: Comparison of heat flux spectra at all measurement locations 


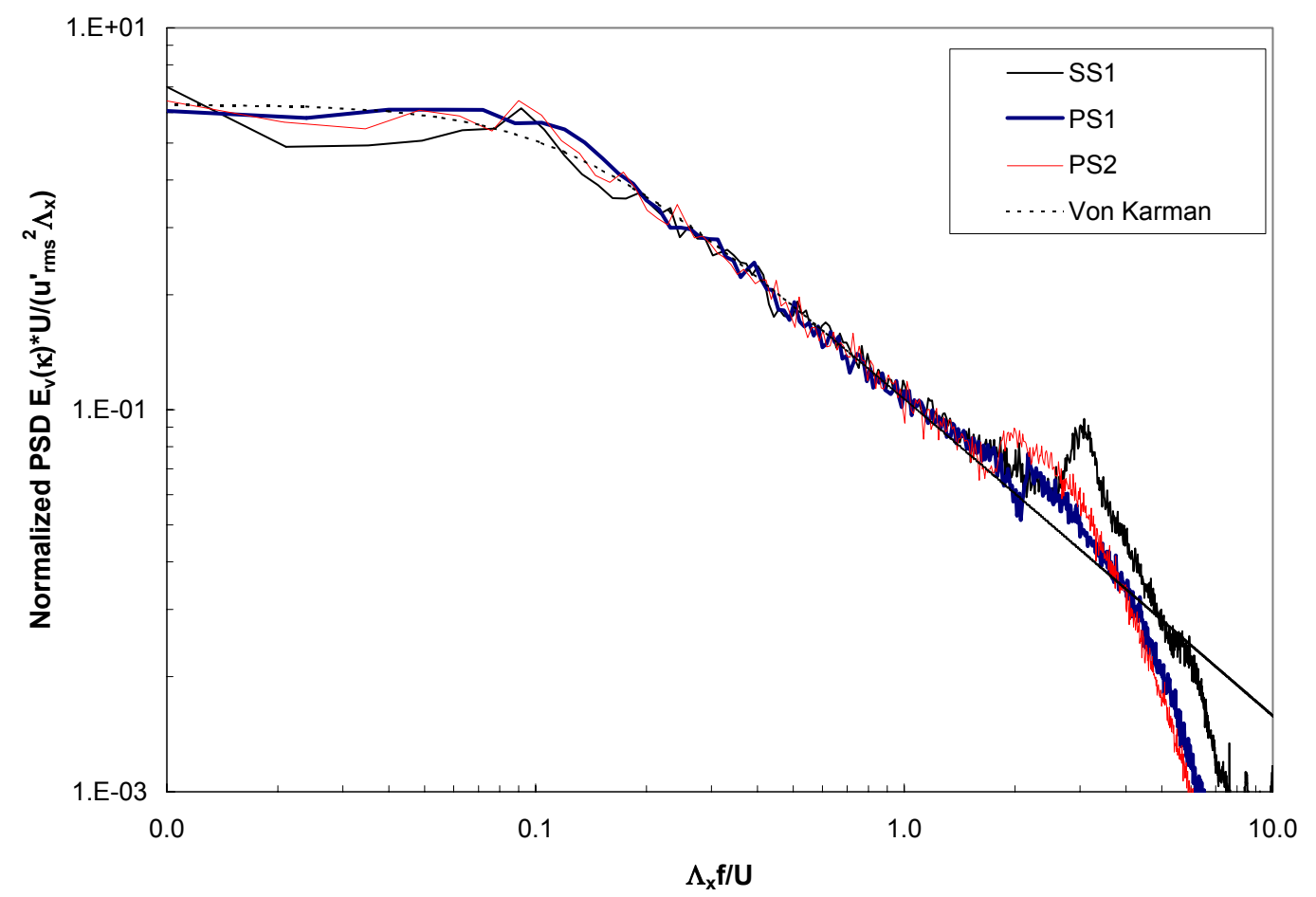

Figure 3.14: Normalized velocity spectra and Von Kármán relation

From Figure 3.11a - Figure 3.11c it can be seen that the velocity and heat flux spectra have very similar shapes and have significant energy at low frequencies which attenuates at frequencies of approximately $5 \mathrm{kHz}$ and above. The spikes in the velocity spectra between 10 and $15 \mathrm{kHz}$ are due to vibration of the hot-wire probe support. Vibration of the hot-wire probe at this frequency would be driven by vortex shedding from the probe support. The shedding frequency of a body can be described by the dimensionless parameter of Strouhal (S),

$$
S=\frac{f_{s} \cdot D}{U}
$$

where $\mathrm{f}_{\mathrm{s}}$ is the vortex shedding frequency, $\mathrm{D}$ is the diameter of the body (probe support), and $\mathrm{U}$ is the mean velocity approaching the probe support. The Strouhal number is a function of the geometry of the body and Reynolds number. For a circular cylinder in cross-flow, the Strouhal number has been demonstrated to be 0.21 (Blevins, 1994). Using a value of $S=0.21$, the mean velocity (U) near the surface of each gage location and 
the probe support diameter (D) of $3.2 \mathrm{~mm}$, the calculated shedding frequency ranges from $9 \mathrm{kHz}$ at PS1 to $14 \mathrm{kHz}$ at SS1. These calculated values agree with results in Figure 3.11. This peak in the spectra is much higher at the suction surface location (SS1) due to higher velocity at this location. The probe vibration is not an issue since it is in a region of the spectrum where most of the energy has been attenuated and does not affect calculations of the RMS value or coherence between the velocity and heat flux signals (discussed in the following section).

The data in Figure 3.12 indicate the relative levels of RMS velocity fluctuations at the three locations (similar to Figure 3.9 in the time domain). As shown, the fluctuating velocity spectrum for the suction surface location (SS1) has much less energy than the two pressure surface locations (PS1 and PS2). This agrees with the data presented in Table 3.3. The spectra were normalized using the local mean velocity, RMS of fluctuating velocity and length scale as shown in Figure 3.14 and compared to the theoretical Von Kármán relation for one-dimensional spectra (Equation 3.11). The data fit well with the Von Kármán equation except for a deviation from the characteristic $-5 / 3$ slope region between 10 and $20 \mathrm{kHz}$ (approximately $\Lambda_{\mathrm{x}} \mathrm{f} / \mathrm{U}$ of 2-3). As mentioned, this is likely due to vibration of the hot-wire probe from vortex shedding.

\subsubsection{Coherence}

As discussed in section 3.1.2.5, correlation of two simultaneously sampled signals in the frequency domain is accomplished through the coherence function. The coherence function was applied to the time-resolved velocity and heat flux data in the frequency domain to determine which frequencies were correlated between the two signals. The coherence function for each gage location is shown in Figure 3.15. 


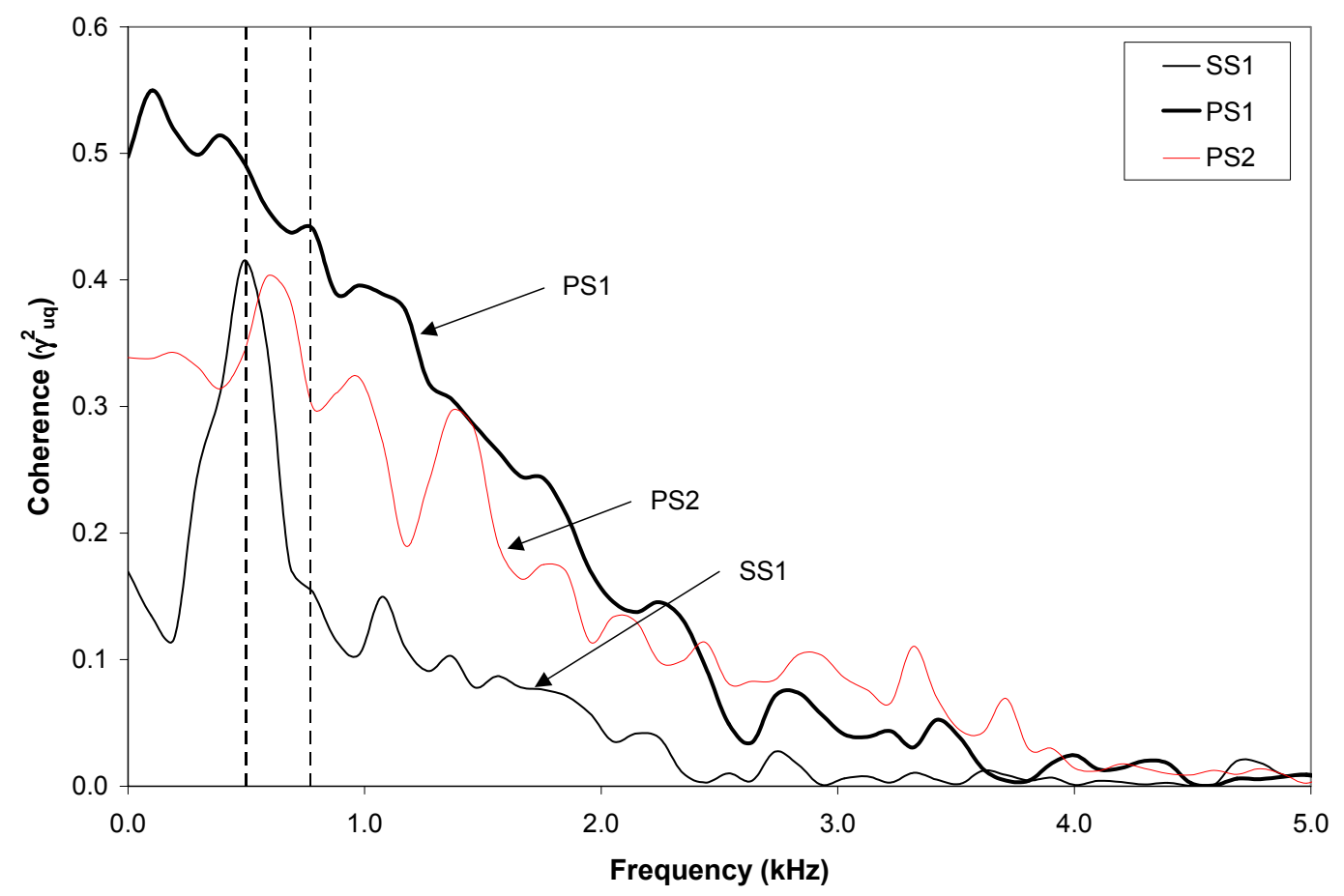

Figure 3.15: Coherence between velocity and heat flux signals

The figure demonstrates that significant coherence at all three gage locations is limited to a relatively narrow band of frequencies below $2 \mathrm{kHz}$ with zero coherence at frequencies starting at about $5 \mathrm{kHz}$. Shown on the figure is a band of frequencies corresponding to the integral length scale range at the three gage locations $\pm 10 \%$. The frequency corresponding to the integral length scale at each location was determined using the analysis presented in section 1.3.2.4 where frequency was converted to the integral scale by $\Lambda_{x}=\frac{3 \cdot \bar{U}}{8 \pi f}$. At each location, the frequency corresponding to the integral length scale was then determined by:

$$
f=\frac{3 \cdot \bar{U}}{8 \pi \Lambda_{x}}
$$

For location SS1, this frequency is approximately $650 \mathrm{~Hz}$, and $570 \mathrm{~Hz}$ and $700 \mathrm{~Hz}$ at locations PS1 and PS2, respectively. 
The frequency range and magnitude of significant coherence agrees very well with data reported by Holmberg (1996). The coherence measured on the pressure surface at PS1 and PS1 shows no "preferential frequencies", rather a broad spectrum of frequencies centered on the integral scale, out to approximately $2 \mathrm{kHz}$, show significant coherence. On the suction surface, the coherence shows a peak at approximately $550 \mathrm{~Hz}$. This peak is well correlated with the frequency of the integral scale. The comparison of the coherence on the two surfaces may indicate that a much smaller range of coherent structures in the freestream penetrate the boundary layer on the suction surface to affect surface heat transfer. On the pressure surface, the range of turbulent vortices in the freestream which show significant coherence with surface heat transfer is broader, possibly indicating the ability of lower energy scales to penetrate into the boundary layer to affect surface heat transfer.

\subsection{Analytical Model}

Using the time-resolved velocity and heat flux data and results (both steady heat transfer and frequency domain results), an analytical model, which may be used to predict increases in steady heat transfer due to freestream turbulence, was developed. The following sections document the development of the model and present results of application of the model to the current data, as well as to data from the work of other researchers on varying geometries and under different flow conditions.

\subsubsection{Analytical Model Development}

The analytical model developed is based on the concept of large-scale eddies penetrating the boundary layer and directly impacting surface heat transfer. Results from analyses of the time-resolved velocity and heat flux data in the time and frequency domains have demonstrated that the large-scale (low frequency) eddies present in the freestream are highly correlated with surface heat transfer. Work by Moss and Oldfield (1994) demonstrated through time-resolved measurements of velocity and heat flux on a flat plate that eddies in the boundary layer convected at the freestream velocity, rather than at the $\sim 0.8 \mathrm{U}$ characteristic of boundary layers. They concluded through time domain and spectral analysis of hot-wire and heat flux gage data and through correlation analysis between heat flux gages that the turbulent eddy structure of the boundary layer is 
dominated by freestream turbulence structures and that heat transfer enhancement is caused by these large eddies being carried deep down into the boundary layer.

The model developed in the current work assumes that freestream eddies penetrate to and directly transfer heat to the surface. The resultant heat transfer is superimposed onto the normal boundary layer heat transfer. The model assumes that the heat transfer process is linear. Figure 3.16 illustrates the idea behind the model.

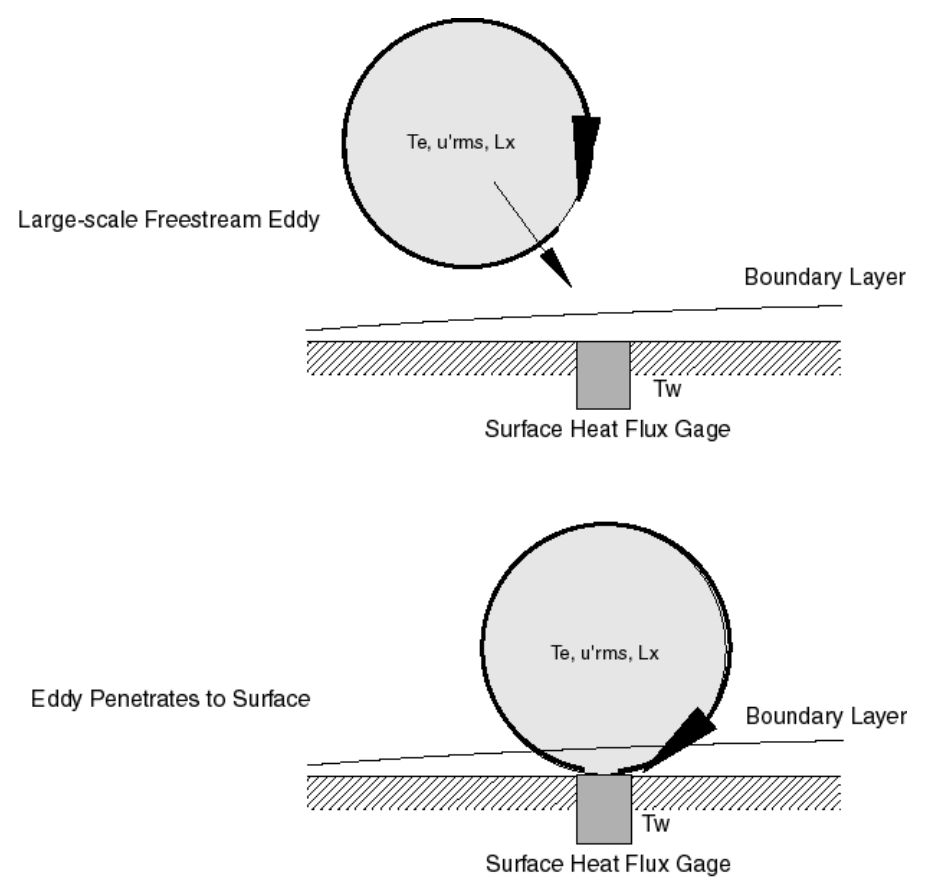

Figure 3.16: Illustration of turbulent heat transfer model

Assuming the eddies have indeed penetrated the thermal boundary layer and reached the surface of the turbine blade, a model of how the heat "packaged" in these eddies is transferred to the surface was needed. The model employs the concept of the semi-infinite medium, whereby a "slug" of hot gas with a temperature representative of the freestream $\left(T_{\infty}\right)$ impacts the surface and resides for a short time, transferring heat to the constant temperature wall $\left(\mathrm{T}_{\mathrm{w}}\right)$ as a semi-infinite medium of gas. The equation for heat transfer for a semi-infinite medium with constant surface temperature is given by (Incropera and DeWitt, 1990): 


$$
q_{s}^{\prime \prime}(t)=-\left.k \frac{\partial T}{\partial x}\right|_{x=0}=k \cdot \frac{\left(T_{\infty}-T_{w}\right)}{\sqrt{\pi \alpha t}}
$$

where $\mathrm{k}$ is the thermal conductivity and $\alpha$ is the thermal diffusivity of the hot gas slug. Assuming that the increase in heat transfer due to freestream turbulence over measured heat transfer without turbulence was due to the combined effect of these eddies enhancing surface heat transfer, equation 3.24 is then equal to:

$$
\Delta q_{t}=k \cdot \frac{\left(T_{e}-T_{w}\right)}{\sqrt{\pi \alpha t}}
$$

where the thermal diffusivity $(\alpha)$ and thermal conductivity $(\mathrm{k})$ are based on the representative eddy temperature, $\mathrm{T}_{\mathrm{e}}$ (static temperature of flow). The model results yield infinite heat flux at time $\mathrm{t}=0$, followed by heat flux which decays as $\frac{1}{\sqrt{t}}$. The increase in heat transfer $\left(\Delta q_{t}\right)$ over low turbulence measurements is determined by averaging $q$ over the time of the event which is estimated as $\mathrm{t}=\Lambda_{\mathrm{x}} / \mathrm{u}_{\text {rms }}^{\prime}$, where $\Lambda_{\mathrm{x}}$ and $\mathrm{u}_{\mathrm{rms}}^{\prime}$ are measured experimentally with a hot-wire. This time is essentially the residence time of a freestream eddy that has penetrated to the surface. Using the values of $\Lambda_{\mathrm{x}}$ and $\mathrm{u}_{\mathrm{rms}}^{\prime}$ to determine this time assumes that all of the energy contained within the freestream turbulence field $\left(\mathrm{u}_{\mathrm{rms}}^{\prime}\right)$ is packed into a representative sized eddy, which for the model the most energetic eddies $\left(\Lambda_{\mathrm{x}}\right)$ were used. The increase in heat transfer coefficient over low turbulence heat transfer is then determined by the following series of equations:

$$
\begin{gathered}
\Delta q_{t}=k \cdot \frac{\left(T_{e}-T_{w}\right)}{\left[\pi \alpha \frac{\Lambda_{x}}{u_{r m s}^{\prime}}\right]^{1 / 2}} \\
\Delta q_{t}=\Delta h_{t} \cdot\left(T_{e}-T_{w}\right)
\end{gathered}
$$




$$
\Delta h_{t}=\frac{k}{\left[\pi \alpha \frac{\Lambda_{x}}{u_{r m s}^{\prime}}\right]^{1 / 2}}
$$

so the increases in heat transfer $\left(\Delta \mathrm{h}_{\mathrm{t}}\right)$ can then be determined through measurements of steady heat transfer at low turbulence conditions $\left(\mathrm{h}_{\mathrm{o}}\right)$ and measurement of the length scale $\left(\Lambda_{\mathrm{x}}\right)$ and RMS of turbulent fluctuations $\left(\mathrm{u}_{\mathrm{rms}}^{\prime}\right)$ along with knowledge of a representative temperature of the flow. The representative eddy temperature could be the static, total or recovery temperature of the flow, but since the eddy temperature is only important in determining the values of the thermal conductivity and diffusivity, variations between these temperatures would only have a small effect on the results of the model calculations. The turbulent heat transfer coefficient could then be determined by adding the increases predicted by the model to the measured heat transfer at low turbulence:

$$
h_{\text {turb }}=h_{o}+\Delta h_{t}
$$

Several key points can be made about the model:

- The model is independent of the geometry of the surface, although both the turbulent fluctuations ( $\left.u_{\text {rms }}^{\prime}\right)$ and the length scale $\left(\Lambda_{\mathrm{x}}\right)$ may be affected by surface geometry.

- The model is independent of the state of the boundary layer. The model does not rely on any knowledge of whether the boundary layer is laminar, transitional or turbulent, since the primary assumption is that the large-scale freestream turbulent structures from the freestream superimpose on the boundary layer heat transfer without high freestream turbulence.

- The model is independent of the boundary layer thickness, but assumes that the eddy scales are much larger than the boundary layer thickness.

- The model assumes that the increase in heat transfer due to turbulence over the existing heat transfer with low freestream turbulence is a linear process (linear superposition). 
These points simply state how the model is applied to predict surface heat transfer. The ability of the model to accurately predict increases in surface heat transfer due to freestream turbulence may be affected by geometry and boundary layer characteristics, however, the model is not a function of these parameters. In the following sections, the analytical model will be applied to the current data set and results presented in sections 3.2.3.1 and 3.2.3.2 and also to the data of other researchers.

\subsubsection{Single-scale Model Results}

The analytical model in the form developed in the previous section is referred to in this text as the "single-scale" model. The model uses integral parameters of the freestream turbulence energy and length scale to determine the increase in steady heat transfer. Later a variation of the model will be developed which uses the frequency domain data and will be referred to as the "multiple-scale" model. The single-scale model was applied to the current data set using measured steady heat transfer coefficients with and without turbulence and the measured turbulence at each gage location. The results are presented below.

\subsubsection{Application to Transonic Facility Data}

Using the measured turbulence data presented in Table 3.3 and Table 3.4 and determining the freestream static temperature at each gage location from the measured test section total temperature and the blade Mach number distribution, the single-scale model was applied to several data runs for each gage location on both the pressure and suction surfaces of the transonic turbine blades. Table 3.5 summarizes the data from Table 3.1 through Table 3.4 and applies the model equation (Equation 3.28) to the data to predict the turbulent heat transfer coefficient at each location. The length scales listed in Table 3.5 compared to the boundary layer thickness estimates detailed in section 3.3 correspond to a ratio of length scale to boundary layer thickness $\left(\Lambda_{\mathrm{x}} / \delta\right)$ of approximately 100 on the suction surface and 20-30 on the pressure surface.

Due to the nature of the measurement techniques in the transonic facility, the steady heat transfer coefficients with and without high freestream turbulence and the time-resolved velocity and heat flux data were measured in separate runs. The steady heat transfer runs were performed with heated flow and were typically of 20-30 seconds 
in length. The runs with the hot-wire in place were performed without heated flow, but with a pre-cooled turbine blade for the driving temperature difference for heat transfer, as discussed earlier. Separating the runs was necessary for several reasons. Besides the desire for unheated flow when performing hot-wire measurements, the run times of the hot-wire tests were of short length to minimize the possibility of breaking the hot-wire which is extremely fragile, especially in such an aggressive flowfield. In addition, in order to measure steady and time-resolved heat transfer simultaneously, both low and high frequency data acquisition were required. Recording data with the two data acquisition systems simultaneously increases the risk of cross talk and noise in the timeresolved data, increasing uncertainty in the data. The data in Table 3.5 then consists of averaged heat transfer coefficient data without turbulence from Table 3.1, averaged turbulent heat transfer coefficient data from Table 3.2 and $\mathrm{u}_{\mathrm{rms}}^{\prime}$ and $\Lambda_{\mathrm{x}}$ data recorded from time-resolved data runs with the hot-wire collocated with the heat flux gage (Table 3.3 and Table 3.4) along with uncertainty in the analytical model predicted increases.

Table 3.5: Results of single-scale model applied to transonic tunnel data

\begin{tabular}{|c|c|c|c|c|c|c|c|}
\hline $\begin{array}{c}\text { Meas. } \\
\text { Location }\end{array}$ & $\begin{array}{c}\text { Measured } \\
\mathbf{u} \mathbf{r m s} \\
\mathbf{m} / \mathbf{s}\end{array}$ & $\begin{array}{c}\text { Measured } \\
\Lambda_{\mathbf{X}} \\
\mathbf{c m}\end{array}$ & $\begin{array}{c}\Delta \mathbf{h}_{\mathbf{t}} \\
\mathbf{W} / \mathbf{m}^{2} \mathbf{K}\end{array}$ & $\begin{array}{c}\text { Low FST } \\
\mathbf{h} \\
\mathbf{W} / \mathbf{m}^{\mathbf{2}} \mathbf{K}\end{array}$ & $\begin{array}{c}\text { Predicted } \\
\text { Turbulent } \\
\mathbf{h} \\
\mathbf{W} / \mathbf{m}^{\mathbf{2}} \mathbf{K}\end{array}$ & $\begin{array}{c}\text { Uncertainty } \\
\text { In Modeled } \\
\mathbf{h} \\
\mathbf{W} / \mathbf{m}^{2} \mathbf{K}\end{array}$ & $\begin{array}{c}\text { Measured } \\
\text { Turbulent } \\
\mathbf{h} \\
\mathbf{W} / \mathbf{m}^{2} \mathbf{K}\end{array}$ \\
\hline \multirow{4}{*}{ SS1 } & 9.31 & 3.81 & 57 & 719 & 776 & 3 & 779 \\
\cline { 2 - 8 } & 9.09 & 3.51 & 58 & 719 & 777 & 3 & 779 \\
\cline { 2 - 8 } & 8.56 & 3.08 & 60 & 719 & 779 & 3 & 779 \\
\hline \multirow{4}{*}{ PS1 } & 20.97 & 2.34 & 116 & 652 & 768 & 5 & 764 \\
\cline { 2 - 8 } & 21.79 & 2.41 & 116 & 652 & 768 & 5 & 764 \\
\cline { 2 - 8 } & 21.30 & 2.34 & 117 & 652 & 769 & 5 & 764 \\
\cline { 2 - 8 } & 21.29 & 2.48 & 114 & 652 & 766 & 5 & 764 \\
\hline \multirow{2}{*}{ PS2 } & 17.47 & 2.54 & 101 & 566 & 667 & 5 & 662 \\
\cline { 2 - 8 } & 16.90 & 2.48 & 100 & 566 & 666 & 5 & 662 \\
\hline
\end{tabular}

The results demonstrate very good agreement between the model predicted and average measured turbulent heat transfer coefficients on both blade surfaces. In addition, the analytical model closely predicts the observed differences in heat transfer 
augmentation between the suction surface and pressure surface. These results have demonstrated that a relatively simple analytical model with a physical basis (penetration of large scale eddies deep into the boundary layer) coupled with a theoretical heat transfer mechanism (semi-infinite medium assumption) is very successful at predicting increases in heat transfer due to freestream turbulence. The model requires only the knowledge of the local turbulence characteristics (velocity fluctuations and scale) near the surface of the turbine blade. In order to further test the validity of the model, its application to additional data sets needs to be explored. The following sections continue the application of the model to the data sets of other researchers.

\subsubsection{Application to Other Data Sets}

With the model data applied to the current data set with extremely good results, the model was then applied to the work of other researchers to test its validity against other data sets. These data sets include several different geometries and both laminar and turbulent boundary layers. According to the primary assumptions of the model, the geometry and state of the boundary layer should not affect the ability of the model to predict increases in heat transfer due to turbulence. Requirements for application of the model were that the experiments measured surface heat transfer coefficients with and without turbulence and included local measurements (i.e. collocated with the surface heat transfer measurements) of time-resolved velocity to determine $\mathrm{u}_{\mathrm{rms}}^{\prime}$ and $\Lambda_{\mathrm{x}}$. Knowledge of the freestream temperature reduces uncertainty in the modeling, but estimated values based on reported data should be adequate, since this quantity only affects the values of the thermal conductivity and thermal diffusivity according to Equation 3.28, which have a second order effect on the model results. The local velocity measurements in these works were performed by either hot-wire anemometry or LDV measurements. The data sets that were used for comparison with the single-scale model were as follows:

- Stagnation point data - Diller and Van Fossen, 1996

- Low speed turbine vane data-Radomsky and Thole, 1998

- Low speed turbine vane pressure surface data - Ames, 1994

- Turbulent flat plate data - Moss and Oldfield, 1992

- Transonic turbine blade data - Holmberg, 1996 
The model application in these cases spans cylinder stagnation point data, flat plat data, turbine vane and turbine blade data, and includes both laminar and turbulent boundary layers on different geometries. The following sections document the application of the model to these cases and present the results, in some cases discussing limitations of the model based on these results.

\subsubsection{Stagnation Point Data - Diller and Van Fossen, 1996}

The effects of freestream turbulence on stagnation point heat transfer, measuring time-resolved velocity and heat flux, were studied in unpublished work by Diller and Van Fossen (1996). The work was performed in the same facility as published work by Van Fossen, et al. (1995), using the same facility, turbulence grids and heat transfer models. The heat transfer models had an elliptical leading edge with a wedge shaped afterbody extending downstream to eliminate vortex shedding. The heat transfer model was placed in a low speed wind tunnel with a freestream velocity of approximately $45 \mathrm{~m} / \mathrm{s}$. The cylinder model had an HFM high frequency heat flux microsensor mounted in the stagnation region and a hot-wire anemometer probe mounted such that it could be traversed in the streamwise direction to measure time-resolved velocity approaching the stagnation region. The turbulence grid was placed upstream of the heat transfer models and consisted of a biplanar grid of $1 / 16$ inch $(0.159 \mathrm{~cm})$ bars spaced $0.72 \mathrm{~cm}$ apart. The results of the heat transfer tests for this grid (G4 in the work of Van Fossen, et al.) are presented in Table 3.6 below.

Table 3.6: Diller (1996) heat transfer data

\begin{tabular}{|c|c|c|c|c|c|c|c|}
\hline Case & $\begin{array}{l}\text { Distance } \\
\text { (in) }\end{array}$ & $\begin{array}{c}\text { Mean } \\
\text { Velocity } \\
(\mathbf{m} / \mathbf{s})\end{array}$ & $\begin{array}{c}\text { Mean } \\
\text { Heat } \\
\text { Flux } \\
\left(\mathbf{W} / \mathbf{m}^{2}\right)\end{array}$ & $\begin{array}{c}\text { RMS } \\
\text { Velocity } \\
(\mathbf{m} / \mathbf{s})\end{array}$ & $\begin{array}{c}\text { Measured } \\
\text { HTC } \\
\left(\mathbf{W} / \mathrm{m}^{2} \mathrm{~K}\right)\end{array}$ & $\begin{array}{l}\text { Tu } \\
\%\end{array}$ & $\begin{array}{c}\text { Lx } \\
\text { (cm) }\end{array}$ \\
\hline G4201 & 1.89 & 32.18 & 4861 & 2.4295 & 262 & 7.55 & 0.86 \\
\hline G4202 & 0.27 & 15.15 & 4925 & 2.3937 & 267 & 15.80 & 0.65 \\
\hline G4203 & 0.22 & 13.31 & 4971 & 2.5867 & 272 & 19.43 & 0.98 \\
\hline G4204 & 0.17 & 11.09 & 5149 & 2.7481 & 271 & 24.78 & 0.77 \\
\hline G4205 & 0.12 & 8.53 & 5403 & 2.6242 & 274 & 30.78 & 0.42 \\
\hline G4206 & 0.07 & 6.06 & 5504 & 2.2664 & 273 & 37.41 & 0.24 \\
\hline
\end{tabular}


The data in Table 3.6 represents six cases where the hot-wire probe was located at different streamwise locations (distance in second column) upstream of the stagnation region of the heat transfer model. The mean velocity decreases as the wire is moved closer to the stagnation region. The streamwise turbulence intensity is normalized by the mean velocity, so the turbulence intensity increases at locations nearer the stagnation region. The streamwise velocity fluctuations are relatively constant approaching the stagnation region of the blade, while the length scale decreases. The analytical model was applied to these data and the predicted heat transfer coefficients with turbulence were compared to measured data. The results are shown in Table 3.7. The mean heat transfer coefficient at low turbulence (less than $0.5 \%$ for this facility) was approximately $180 \mathrm{~W} / \mathrm{m}^{2} \mathrm{~K}$. Uncertainty in the model predictions is likely of the same order as the model predictions from the current research work $\left( \pm 4.5 \%\right.$ on the value of $\left.\Delta \mathrm{h}_{\mathrm{t}}\right)$.

As the results show, the model predictions of the turbulent heat transfer coefficient are quite good. The location where the turbulence characteristics are measured closest to the stagnation region ( 0.07 inches) provides the best prediction of heat transfer increases due to turbulence, indicating the dependence of the model on local turbulence measurements, rather than measurements far upstream. In the case of a flat plate or turbine vane or blade, this would translate to measurements near the surface, close to the boundary layer edge, rather than far out into the freestream.

Table 3.7: Results of single-scale model applied to Diller (1996) data

\begin{tabular}{|c|c|c|c|c|c|c|}
\hline Case & $\begin{array}{c}\text { Measured } \\
\text { u'rms } \\
(\mathbf{m} / \mathbf{s})\end{array}$ & $\begin{array}{c}\text { Measured } \\
\Lambda_{\mathbf{x}} \\
(\mathrm{cm})\end{array}$ & $\begin{array}{c}\Delta \mathbf{h}_{\mathrm{t}} \\
\left(\mathrm{W} / \mathbf{m}^{2} \mathrm{~K}\right)\end{array}$ & $\begin{array}{c}\text { Low } \\
\text { FST } \\
\text { h } \\
\left(\mathrm{W} / \mathbf{m}^{2} \mathrm{~K}\right)\end{array}$ & $\begin{array}{c}\text { Predicted } \\
\text { Turbulent } \\
\mathbf{h} \\
\left(\mathrm{W} / \mathbf{m}^{2} \mathbf{K}\right)\end{array}$ & $\begin{array}{c}\text { Measured } \\
\text { Turbulent } \\
\quad \mathbf{h} \\
\left(\mathrm{W} / \mathbf{m}^{2} \mathbf{K}\right)\end{array}$ \\
\hline G4201 & 2.4295 & 0.86 & 52.56 & 180 & 233 & 262 \\
\hline G4202 & 2.3937 & 0.65 & 60.01 & 180 & 240 & 267 \\
\hline G4203 & 2.5867 & 0.98 & 50.80 & 180 & 231 & 272 \\
\hline G4204 & 2.7481 & 0.77 & 59.07 & 180 & 239 & 271 \\
\hline G4205 & 2.6242 & 0.42 & 78.16 & 180 & 258 & 274 \\
\hline G4206 & 2.2664 & 0.24 & 96.09 & 180 & 276 & 273 \\
\hline
\end{tabular}




\subsubsection{Low speed turbine vane data - Radomsky and Thole, 1998}

The effect of freestream turbulence intensity and length scale on turbine vane heat transfer in a low speed facility was studied in detail by Radomsky and Thole (1998). The study focused on documenting the surface heat transfer and highly turbulent flowfield around a stator vane placed in a large-scale wind tunnel. The test section consists of a stator vane and the adjacent blade leading edges (forming two vane passages) to correctly simulate the pressure distribution and secondary flows about the vane. An active turbulence-generating grid was placed upstream of the test section to simulate the turbulence. Three-component laser Doppler velocimetry (LDV) measurements were performed, mapping the turbulence intensity in the two passages. The turbulent length scale was measured using a single-sensor hot-wire. In order to accurately apply the analytical model, the measurements nearest to the vane surface (near the edge of the boundary layer) were used as input data. Two inlet turbulence conditions $(10 \%$ and $20 \%)$ were compared. The integral length scale of the inlet turbulence was $4.6 \mathrm{~cm}$ for the $10 \%$ turbulence case and $5.0 \mathrm{~cm}$ for the $20 \%$ turbulence case. Local length scale measurements (matching the LDV measurement locations) were not performed, but measurements along a streamline in the center of the vane passage demonstrated that the length scale did not change significantly throughout the vane passages. A constant length scale was then assumed for the model application. The model was used to predict the increase in heat transfer due to turbulence by Equation 3.28, then the value of $\Delta \mathrm{h}_{\mathrm{t}}$ was added to the measured Stanton number for each chord wise location on the vane. The results of the model application are shown graphically in Figure 3.17 and 3.18 with uncertainty bars of $\pm 4.5 \%$ added to selected data points for the values of the Stanton number and $\pm 2.4 \%$ applied to the $\mathrm{St} / \mathrm{St}_{\mathrm{o}}$ data. 


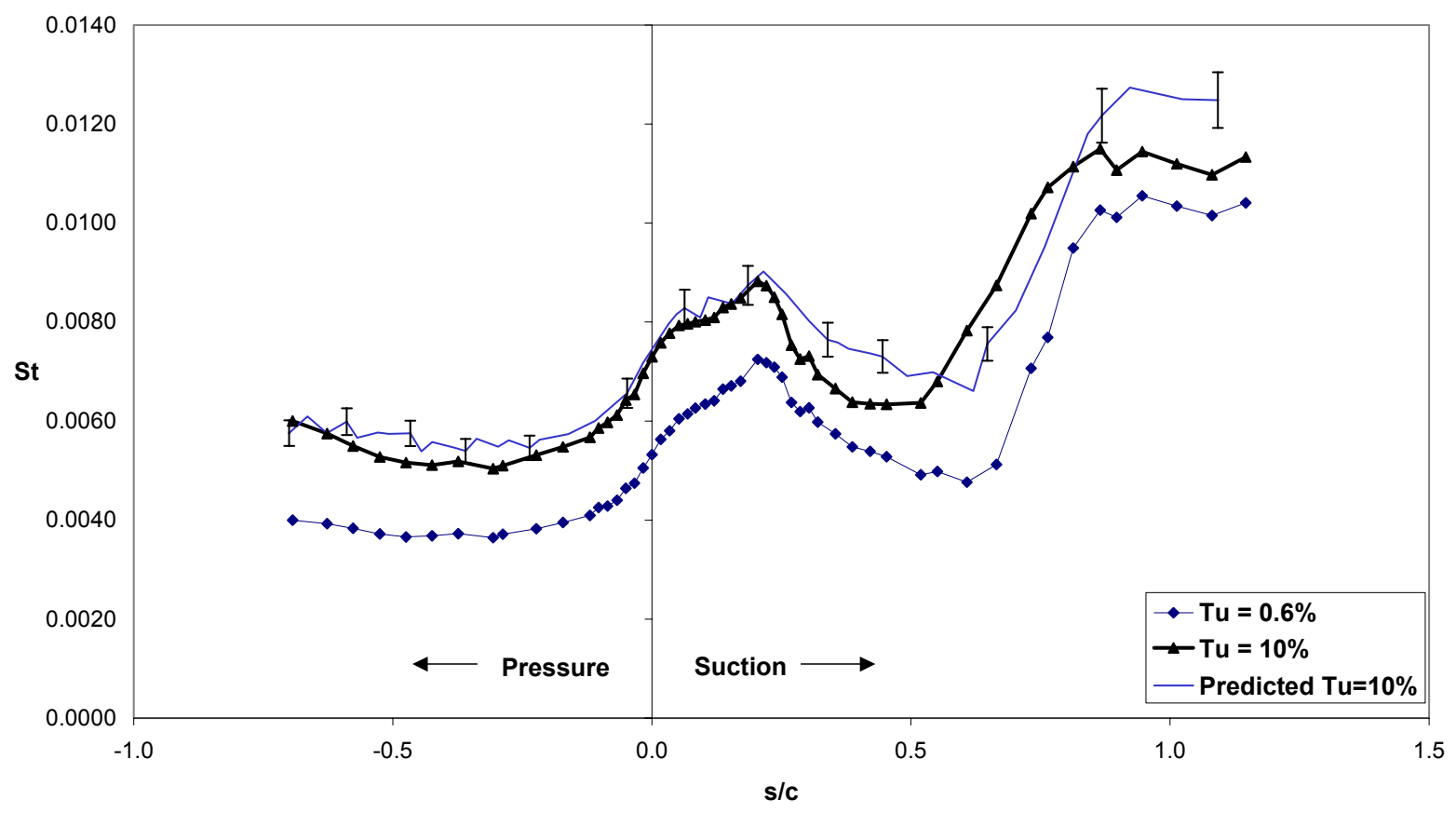

a. $10 \%$ Turbulence Case

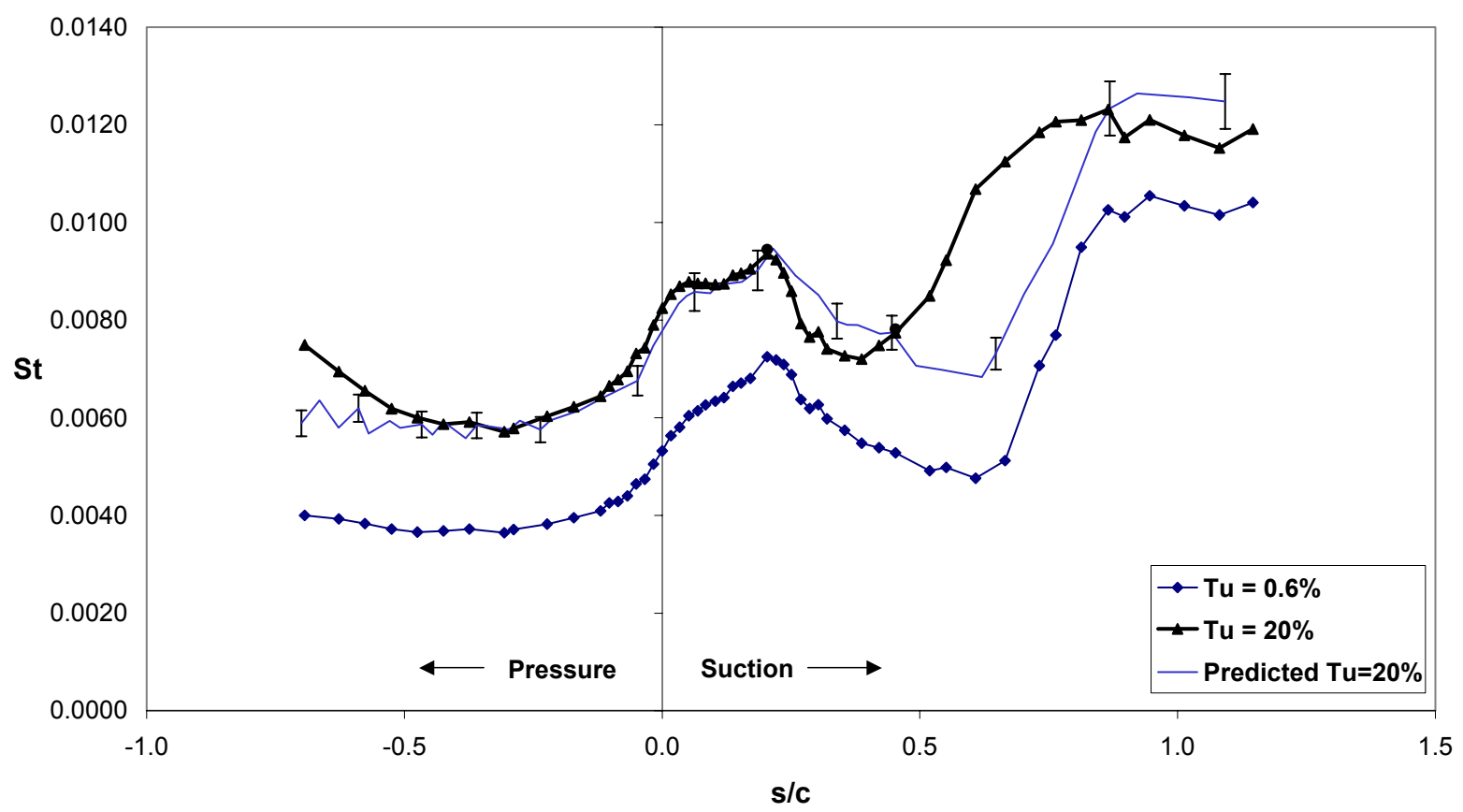

b. $20 \%$ Turbulence Case

Figure 3.17: Comparison of model predicted and measured Stanton numbers (St) from Radomsky and Thole (1998) 


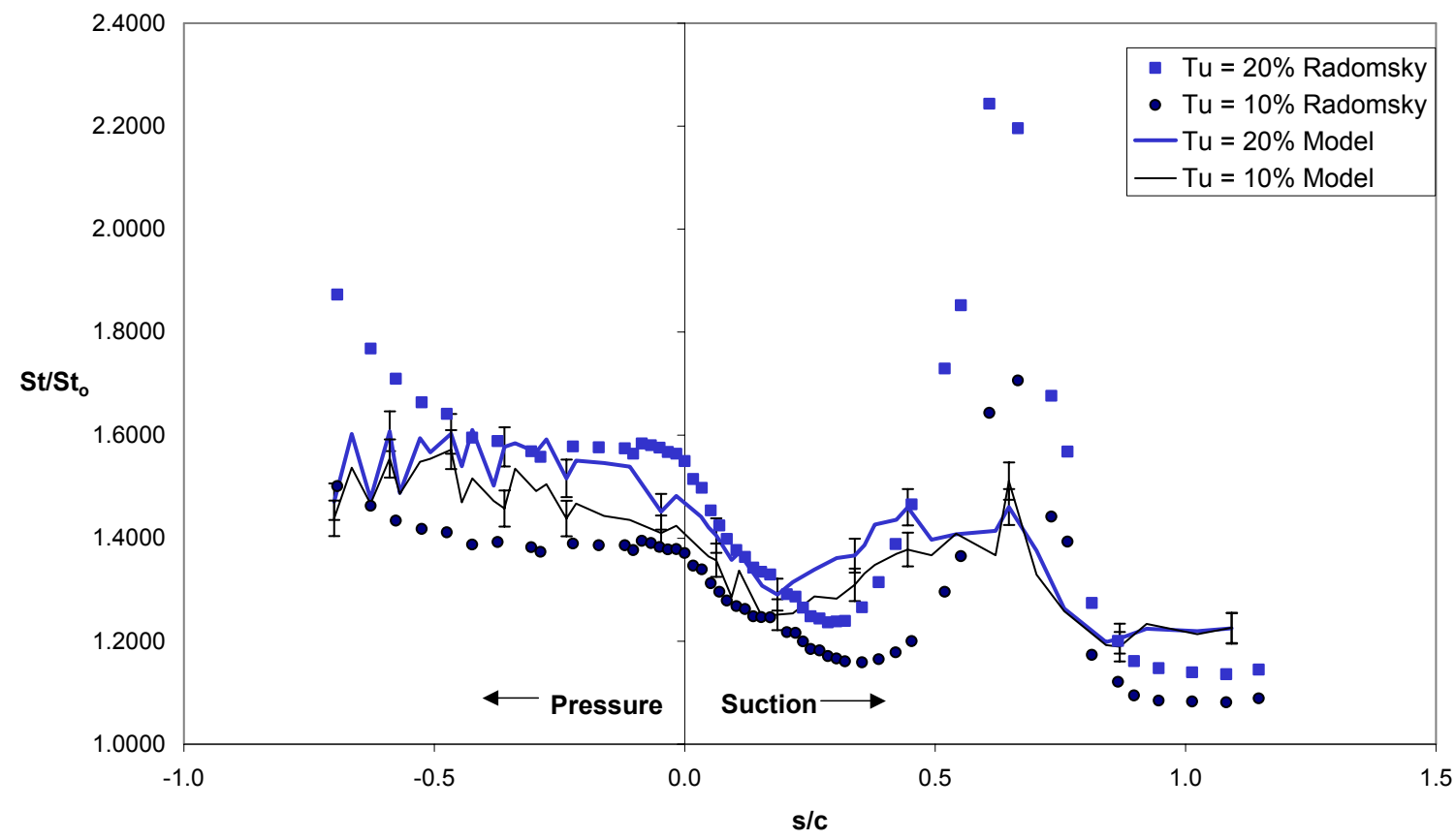

\section{Figure 3.18: Comparison of model predicted and measured $\mathrm{St} / \mathrm{St}_{\mathbf{0}}$ from Radomsky and Thole (1998)}

The results shown in Figure 3.17 demonstrate that the model is fairly good at predicting the increases in heat transfer due to turbulence for both the low $(10 \%)$ and high $(20 \%)$ turbulence cases in the region where the boundary layer is laminar. The model slightly over predicts the heat transfer for the $10 \%$ turbulence case and slightly under predicts the heat transfer for the $20 \%$ turbulence case over most of the blade surface. In both cases, there is a large discrepancy between the model predicted and measured heat transfer on the suction surface at approximately $\mathrm{s} / \mathrm{c}=0.6$ and greater. The boundary layer is transitional and then fully turbulent above this value. The large scale eddies in the flow may interact with the larger scale turbulent eddies in the turbulent boundary layer, reducing their effectiveness at increasing heat transfer. The model does not account for any interaction of scales and would thus over predict the measured heat transfer. Similarly, on the pressure surface for the $20 \%$ turbulence case, the measured heat transfer indicates possible boundary layer transition at $\mathrm{s} / \mathrm{c}=-0.5$ and above and the model predictions are lower than the measured heat transfer and does not predict the turbulence induced transition. In both of these regions, the results demonstrate the 
inability of the model to predict early transition due to turbulence and over prediction of heat transfer in the fully turbulent boundary layer region.

The heat transfer predictions with turbulence are compared to the measured values normalized by the low turbulence heat transfer $\left(\mathrm{St}_{\mathrm{St}}\right)$ in Figure 3.18. The results in the figure clearly demonstrate that the model is unable to predict early transition due to turbulence, since the measured $\mathrm{St} / \mathrm{St}_{\mathrm{o}}$ results show a large peak where heat transfer increases are quite large. The figure also shows that the results predicted by the model for $10 \%$ and $20 \%$ turbulence are not greatly different from one another and are both inside the "envelope" of the measured heat transfer for the two turbulence conditions over most of the blade surface. This further illustrates the under prediction of the $20 \%$ turbulence measurements and the over prediction of the $10 \%$ turbulence measurements. The relatively small difference in the modeled heat transfer increases is a result of the change in the value of $\mathrm{u}_{\mathrm{rms}}^{\prime}$ being offset by the slightly larger length scale for the $20 \%$ turbulence case, since Equation 3.28 which leads to:

$$
\Delta h_{t}=\frac{k}{\left[\pi \alpha \frac{\Lambda_{x}}{u_{r m s}^{\prime}}\right]^{1 / 2}}=\frac{k}{\sqrt{\pi \alpha}}\left(\frac{u_{r m s}^{\prime}}{\Lambda_{x}}\right)^{1 / 2}
$$

The model predictions are sensitive to both $\mathrm{u}_{\mathrm{rms}}^{\prime}$ and $\Lambda_{\mathrm{x}}$ as evidenced by Equation 3.29. Overall, the model is shown to adequately predict increases in heat transfer, with some limitations.

\subsubsection{Low speed turbine vane pressure surface data - Ames, 1994}

Work by Ames (1994) also measured increases in heat transfer due to turbulence on a turbine vane geometry. The work was performed in a low speed wind tunnel with several different turbulence generators. Turbulence is generated using a combustor simulator and using a passive biplanar grid. The combustor simulator was located at two different distances upstream of the vane cascade to give different turbulence levels. The turbulence conditions are referred to by their generator configuration, where the gridgenerated turbulence (Grid) has an intensity of $7.5 \%$ and length scale of $1.1 \mathrm{~cm}$ at the cascade inlet, the combustor simulator closest to the vane passages (Comb 1) generates 
inlet turbulence with an intensity of approximately $13 \%$ and $1.6 \mathrm{~cm}$ length scale and the combustor simulator located further from the cascade (Comb 2) generates inlet turbulence with $8.5 \%$ intensity and $2.1 \mathrm{~cm}$ length scale.

Measurements of local turbulent fluctuations and length scale were performed using a hot-wire probe traversed perpendicular to the turbine vane at four locations on the pressure surface. Only three of these locations were used in the model application, since the fourth location is at the trailing edge of the vane and heat transfer measurements were reported by Ames to have large uncertainties. The data presented in Ames (1994) reported measured turbulent fluctuations and length scale at varying distances from the surface of the blade. The data for the point corresponding to the edge of the boundary layer was used for the model input. The model calculated values of $\Delta \mathrm{h}_{\mathrm{t}}$ were determined and added to the measured heat transfer under low turbulence conditions. Figure 3.19 and Figure 3.20 present the results of the model predictions compared to the measured values with $\pm 4.5 \%$ uncertainty on the modeled data for Stanton number and $\pm 2.4 \%$ uncertainty on the $\mathrm{St} / \mathrm{St}_{\mathrm{o}}$ data.

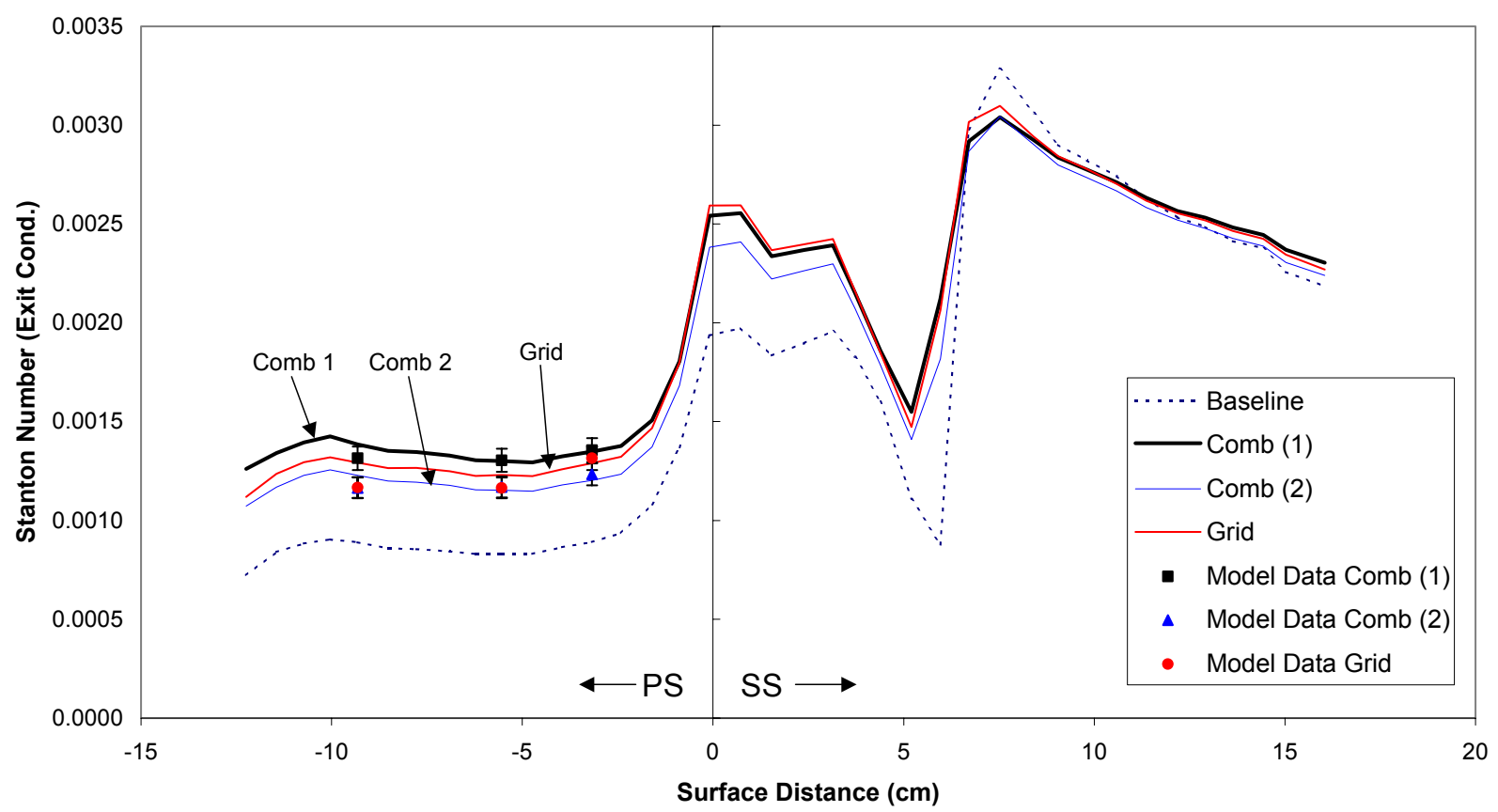

Figure 3.19: Comparison of model predicted and measured Stanton numbers (St) from Ames (1994) 


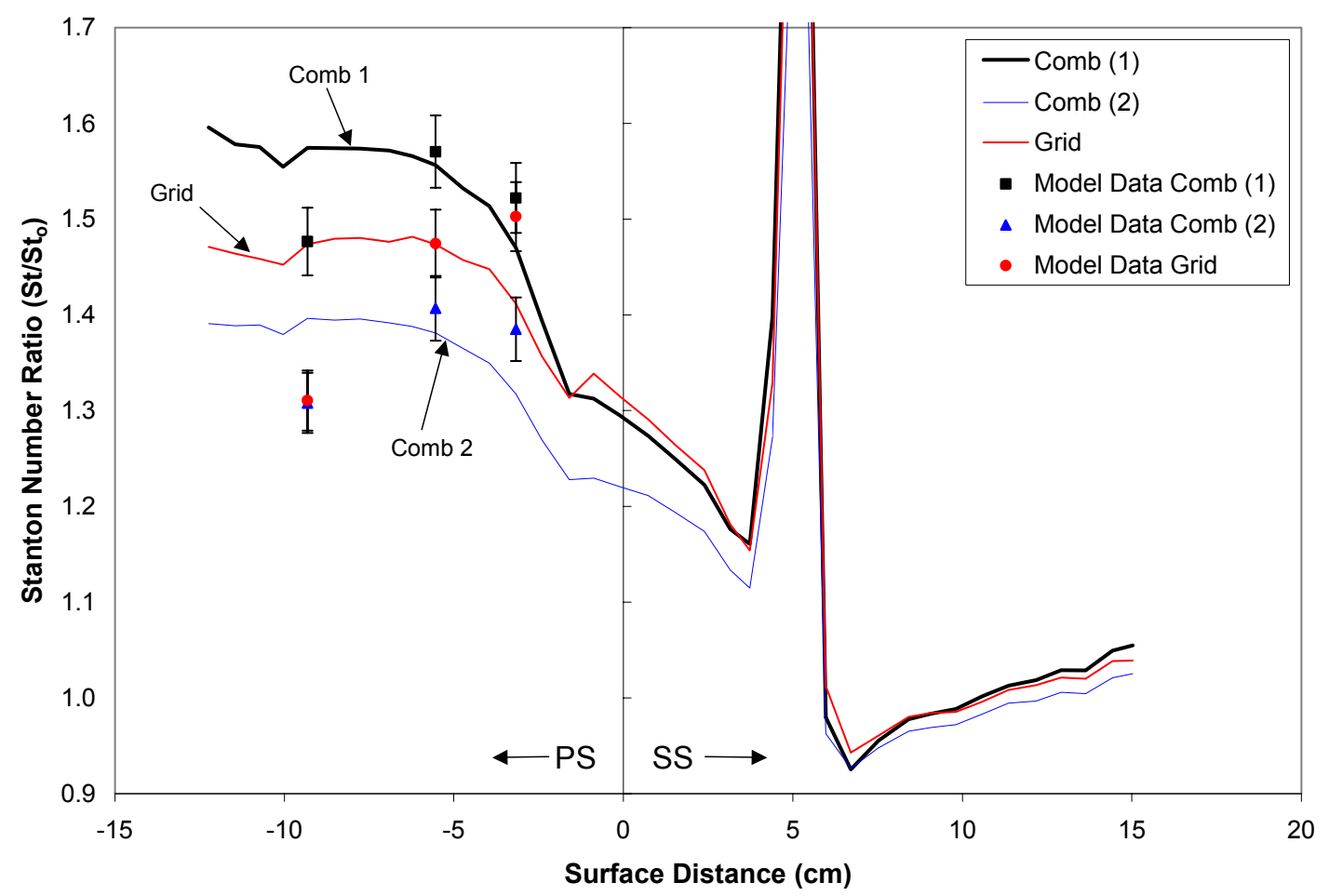

Figure 3.20: Comparison of model predicted and measured $\mathrm{St} / \mathrm{St}_{\mathbf{~}}$ from Ames (1994)

Figure 3.19 indicates that the model is fairly good at predicting the heat transfer due to freestream turbulence. With so few data points to compare with (as opposed to the comparisons performed with the work of Radomsky and Thole (1998)), the results do not give a strong indication as to the ability of the model to accurately predict heat transfer increases. There does appear to be a fairly large discrepancy in the model results and the measured data at the location furthest along the pressure surface. As indicated by Ames (1994), locations far along the blade surface (near the trailing edge) have larger measurement uncertainties.

Figure 3.20 plots the model results against measured data for the heat transfer with freestream turbulence normalized by the low turbulence value $\left(\mathrm{St} / \mathrm{St}_{\mathrm{o}}\right)$. The large difference in the furthest streamwise measurement location is well illustrated in this case. For the other two measurement locations, the model is shown to over predict the turbulent heat transfer. In general, the model does not appear to well predict the Comb 2 case. Based on reported uncertainty in the measured data, error bars were generated for the measured heat transfer data for comparison with the model data using the perturbation 
technique described in Moffat (1988). Figure 3.21 plots the data from Comb 2 with several error bars on the curve along with the model predicted data. As shown, the data fall within the uncertainty in the measurement of the value of the normalized heat transfer data $\left(\mathrm{St} / \mathrm{St}_{\mathrm{o}}\right)$.

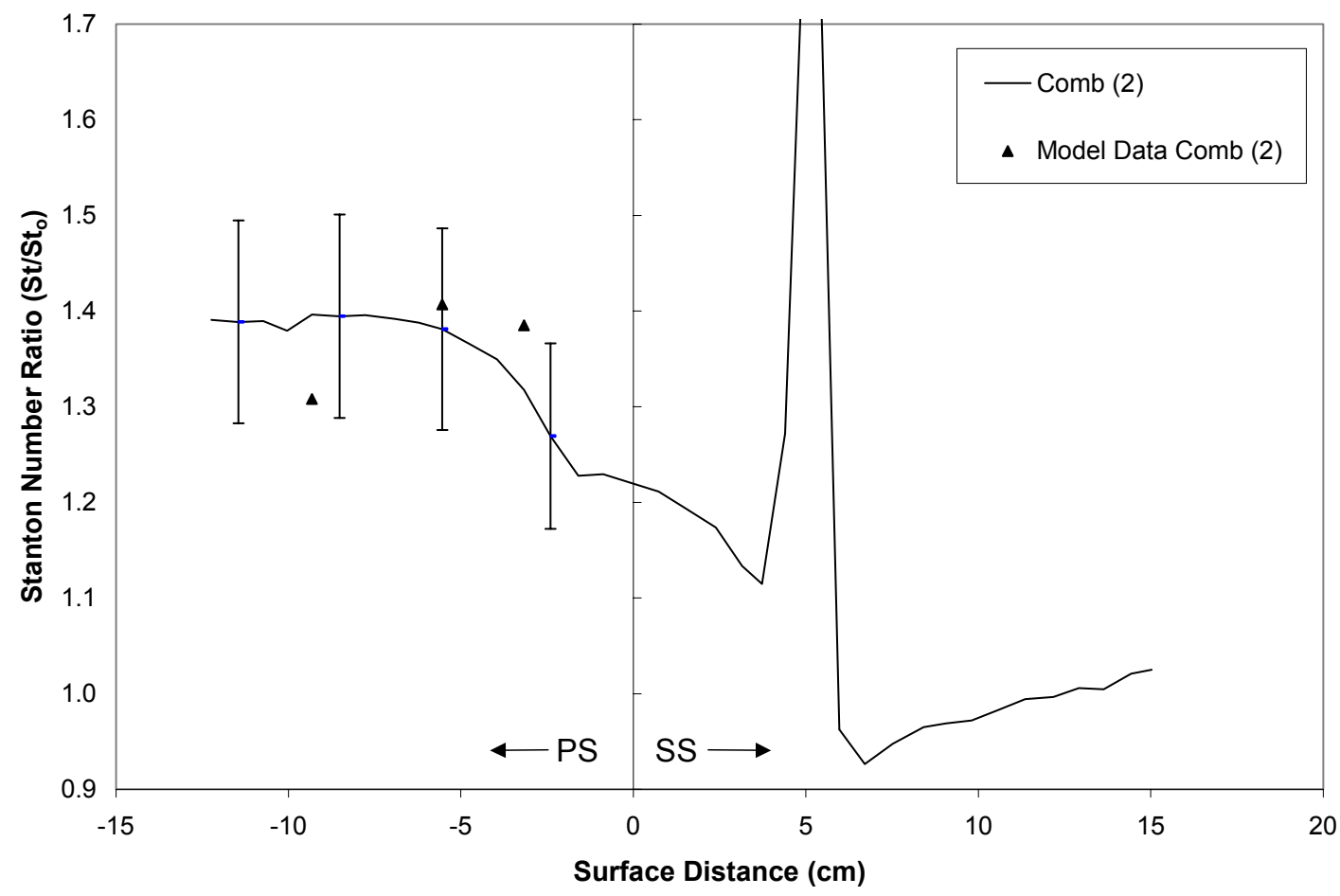

Figure 3.21: Comparison of model predicted and measured $\mathrm{St}_{/} \mathrm{St}_{\mathbf{0}}$ from $\mathrm{Ames}$ (1994) for Comb 2 with error estimates

\subsubsection{Turbulent flat plate data - Moss and Oldfield, 1992}

Measurements of the effect of freestream turbulence and length scale on heat transfer to a flat plate with a turbulent boundary layer were reported by Moss and Oldfield (1992). Turbulence was generated by a series of upstream turbulence grids of varying bar diameter and pitch. Heat transfer was measured at three streamwise locations on the surface of the plate with and without freestream turbulence. Fluctuating velocity was measured with a hot-wire probe near the edge of the boundary layer at the surface of the plate. The flat plate boundary layer was tripped to create a turbulent boundary layer at downstream locations. The heat transfer due to freestream turbulence was modeled, similar to previous cases, by adding the value of $\Delta \mathrm{h}_{\mathrm{t}}$ to the low freestream turbulence heat 
transfer measurements reported in the work. For this comparison, the grids with the highest intensity were used (Grids D and E in Moss and Oldfield (1992)) for the comparisons. The results of the modeling are presented in Table 3.8. Uncertainty in the modeled values of $\mathrm{Nu} / \mathrm{Nu}_{\mathrm{o}}$ was estimated through the perturbation method (Appendix B) to be $\pm 2.4 \%\left( \pm 0.03 \mathrm{Nu} / \mathrm{Nu}_{\mathrm{o}}\right)$.

Table 3.8: Model results compared to data from Moss and Oldfield (1992)

\section{Grid D}

\begin{tabular}{|c|c|c|c|c|c|c|c|}
\hline $\begin{array}{c}\text { Dist. } \mathbf{x} \\
(\mathbf{m m})\end{array}$ & $\begin{array}{c}\mathbf{T u} \\
\mathbf{( \% )}\end{array}$ & $\begin{array}{c}\mathbf{u}^{\prime}{ }_{\mathbf{r m s}} \\
\mathbf{( m / s )}\end{array}$ & $\begin{array}{c}\Lambda_{\mathbf{x}} \\
(\mathbf{c m})\end{array}$ & $\begin{array}{c}\Delta \mathbf{h}_{\mathbf{t}} \\
\left(\mathbf{W} / \mathbf{m}^{2} \mathbf{K}\right)\end{array}$ & $\begin{array}{c}\mathbf{h}_{\mathbf{o}} \\
\left(\mathbf{W} / \mathbf{m}^{\mathbf{2}} \mathbf{K}\right)\end{array}$ & $\begin{array}{c}\text { Calc. } \\
\mathbf{N u} / \mathbf{N u}_{\mathbf{0}}\end{array}$ & $\begin{array}{c}\text { Reported } \\
\mathbf{N u} / \mathbf{N u}_{\mathbf{0}}\end{array}$ \\
\hline 100 & 16.0 & 11.2 & 1.46 & 86.64 & 210.4 & 1.41 & 1.30 \\
\hline 180 & 15.0 & 10.5 & 1.53 & 81.95 & 182.6 & 1.45 & 1.39 \\
\hline 300 & 11.7 & 8.2 & 1.66 & 69.48 & 157.8 & 1.44 & 1.41 \\
\hline
\end{tabular}

\section{Grid E}

\begin{tabular}{|c|c|c|c|c|c|c|c|}
\hline $\begin{array}{c}\text { Dist. } \mathbf{x} \\
(\mathbf{m m})\end{array}$ & $\begin{array}{c}\mathbf{T u} \\
\mathbf{( \% )}\end{array}$ & $\begin{array}{c}\mathbf{u}_{\text {rms }}^{\prime} \\
\mathbf{( m / s )}\end{array}$ & $\begin{array}{c}\Lambda_{\mathbf{x}} \\
(\mathbf{c m})\end{array}$ & $\begin{array}{c}\Delta \mathbf{h}_{\mathbf{t}} \\
\left(\mathbf{W} / \mathbf{m}^{2} \mathbf{K}\right)\end{array}$ & $\begin{array}{c}\mathbf{h}_{\mathbf{o}} \\
\left(\mathbf{W} / \mathbf{m}^{\mathbf{2}} \mathbf{K}\right)\end{array}$ & $\begin{array}{c}\text { Calc. } \\
\mathbf{N u} / \mathbf{N u}_{\mathbf{0}}\end{array}$ & $\begin{array}{c}\text { Reported } \\
\mathbf{N u} / \mathbf{N u}_{\mathbf{0}}\end{array}$ \\
\hline 100 & 12.1 & 8.5 & 0.85 & 98.75 & 210.4 & 1.47 & 1.30 \\
\hline 180 & 9.4 & 6.6 & 0.97 & 81.47 & 182.6 & 1.45 & 1.30 \\
\hline 300 & 6.9 & 4.8 & 1.21 & 62.50 & 157.8 & 1.40 & 1.30 \\
\hline
\end{tabular}

For grid D, the model predicts the measured increases in heat transfer fairly well, especially at the locations farthest downstream. The first column is the streamwise distance along the plate measured from the leading edge. The model slightly over predicts the measured heat transfer. The model does not predict the heat transfer for grid E as well, over predicting the measured heat transfer at all three streamwise locations. This is similar to the results reported earlier in the comparison with the work of Radomsky and Thole (1998) where heat transfer in the regions of the turbine vane where the boundary layer was turbulent was over predicted by the model. As mentioned earlier, this may be due to the large scale eddies in the boundary layer interacting with the eddies from the freestream, reducing the effectiveness of the large scale eddies in increasing surface heat transfer. 
Of interest is the apparent correlation of the error in the predicted measurements with length scale. The over prediction of measured heat transfer increases as the length scale decreases, even though the turbulence intensity is decreasing. This suggests that large-scale turbulence is well predicted in its effects on turbulent heat transfer. This may indicate that the primary assumption of the model that large-scale eddies penetrate to the surface to affect heat transfer does not hold as well for smaller scale turbulence. In later work by the authors, Moss and Oldfield (1994), the convective velocity of eddy structures in the boundary layer was measured through spatial correlation of high frequency heat flux gages at different locations. It was shown that the convective velocity normalized by the freestream velocity was near 1.0 for grid $\mathrm{D}$, whereas the value was slightly lower (around 0.9) for Grid E, which has smaller length scale. These results lend credence to the possibility that smaller scale freestream turbulence does not completely penetrate the boundary layer, and thus does not affect surface heat transfer to the extent that the model would predict, especially for a turbulent boundary layer.

The authors (Moss and Oldfield (1994)) state that, "The main heat transfer enhancing mechanism is due to the penetration of freestream turbulent eddies deep into the boundary layer, rather than enhancement of existing boundary layer turbulence." From this statement and the results of the analytical model application, it appears that this holds true when the eddies are large enough and have sufficient energy (intensity) to penetrate the boundary layer. The current results (including the comparison with the work of Radomsky and Thole (1998)) suggest that for turbulent boundary layers, the eddies may not completely penetrate to the surface unless the freestream eddies are of sufficient intensity and scale, leading to the over prediction of results for turbulent boundary layers.

\subsubsection{Transonic turbine blade data - Holmberg, 1996}

The final data set analyzed was data from Holmberg (1996) in the same transonic turbine cascade facility as this work. The data was taken with a hot-wire co-located with a heat flux gage on both the pressure and suction surfaces of the transonic turbine blade. Two different turbulence grids were used. The hot-wire probe was located approximately $1 \mathrm{~mm}$ above the blade surface. As discussed in section 1.3, the work of Holmberg was in a turbulence flowfield with much lower turbulence intensity (less than 5\%) and smaller 
length scale $(1-5 \mathrm{~mm})$. Table 3.9 presents the results of the model applied to the data for both grids, with measurements at four locations on the blade surface. The locations on the pressure surface were performed with insert heat flux gages (similar to the ones used in the current work), while the suction surface gage was directly sputtered on the blade surface. For the results reported for gage DS1, the grids were moved to different locations to give different turbulence levels (G\#u and G\#b).

Table 3.9. Model results compared data from Holmberg (1996)

\begin{tabular}{|c|c|c|c|c|c|c|c|c|}
\hline Location & Grid & $\begin{array}{l}\text { Tu } \\
(\%)\end{array}$ & $\begin{array}{l}\mathbf{u}^{\prime}{ }_{\mathrm{rms}} \\
(\mathrm{m} / \mathbf{s})\end{array}$ & $\begin{array}{c}\Lambda_{\mathbf{x}} \\
(\mathbf{m m})\end{array}$ & $\begin{array}{c}\Delta \mathbf{h}_{\mathrm{t}} \\
\left(\mathbf{W} / \mathbf{m}^{2} \mathbf{K}\right)\end{array}$ & $\begin{array}{c}\mathbf{h}_{\mathbf{0}} \\
\left(\mathbf{W} / \mathbf{m}^{2} \mathbf{K}\right)\end{array}$ & $\begin{array}{c}\text { Predicted } \\
\text { Turbulent } \\
\text { h }\end{array}$ & $\begin{array}{c}\text { Measured } \\
\text { Turbulent } \\
\text { h } \\
\end{array}$ \\
\hline \multirow[t]{4}{*}{ DS1 } & G2b & 2.41 & 4.09 & 2.37 & 179 & 1000 & 1179 & 1580 \\
\hline & G2u & 3.15 & 5.33 & 1.79 & 235 & 1000 & 1235 & 1785 \\
\hline & G4b & 1.84 & 3.36 & 4.34 & 119 & 1000 & 1119 & 1360 \\
\hline & G4u & 2.72 & 4.91 & 3.59 & 159 & 1000 & 1159 & 1507 \\
\hline \multirow[t]{2}{*}{ PS1 } & G2 & 4.57 & 4.74 & 1.52 & 247 & 800 & 1047 & 1085 \\
\hline & G4 & 7.15 & 6.94 & 3.92 & 186 & 800 & 986 & 970 \\
\hline \multirow[t]{2}{*}{ PS2 } & G2 & 4.66 & 4.29 & 2.64 & 183 & 820 & 1003 & 930 \\
\hline & G4 & 5.61 & 5.10 & 3.71 & 167 & 820 & 987 & 875 \\
\hline \multirow[t]{2}{*}{ PS3 } & G2 & 2.35 & 2.96 & 1.62 & 188 & 845 & 1033 & 1005 \\
\hline & G4 & 3.07 & 3.91 & 2.50 & 173 & 845 & 1018 & 925 \\
\hline
\end{tabular}

The results demonstrate that the single-scale model predicts the increase in heat transfer reasonably well for the pressure surface locations, while the predicted results for the suction surface are considerably lower than the measured values. This is possibly due to errors in the measurement of heat transfer with the direct sputtered gage. Reported uncertainties in the data were quite high, with error as high as $10-15 \%$ on both surfaces. The uncertainty in the modeled values of $\Delta \mathrm{h}_{\mathrm{t}}$ was estimated to be $4.5 \%$.

\subsubsection{Film-cooled Transonic turbine blade data - Smith, et al., 2000}

In addition to applying the analytical model to data sets that measured heat transfer coefficients with and without turbulence, the model was applied to film-cooled heat transfer data from the work of several researchers at Virginia Tech on the same geometry as the blades in the current work. The work of Smith, et al. (2000), Smith 
(1999), Popp (1999) and Bubb (1999) investigated the effects of film cooling on heat transfer coefficients and film cooling effectiveness on the suction surface of a transonic turbine blade. Measurements were performed at 6 locations on the suction surface of the blade. Measurements of time-resolved velocity were only performed at one gage location on the suction surface (location SS1 in this work, which is location SS2 in the previous work). With high freestream turbulence, the increase in heat transfer coefficient $\left(\Delta \mathrm{h}_{\mathrm{t}}\right)$ for this gage location based on local measurements of turbulence intensity and length scale was determined to be an average of $59 \mathrm{~W} / \mathrm{m}^{2} \mathrm{~K}$ (see Table 3.5). In order to apply the model to estimate the heat transfer coefficient with film-cooling and high freestream turbulence, this value was applied to each of the gage locations from the previous work. This assumes the turbulence intensity and scale is constant in the streamwise direction in the range of these gage locations. This may not be the case, but it is the only available measurement from the current work. One of the primary model assumptions is that the state of the boundary layer is not a factor in the analytical model, thus the model would be applicable to a film-cooling layer as well as normal boundary layer flow. Measurements with film cooling were not performed in this work, but are recommended for future study in this facility. This analysis provides an estimate of what may be expected.

Using the measured film-cooled heat transfer coefficients $\left(\mathrm{h}_{\mathrm{o}, \mathrm{c}}\right)(\mathrm{Bubb}, 1999)$, the predicted heat transfer coefficient with film-cooling and high freestream turbulence was determined by adding the value of $\Delta \mathrm{h}_{\mathrm{t}}$ to the measured film-cooled heat transfer coefficient. Table 3.10 below reports the predicted results. As a comparison with this data, if the low, turbulence no film-cooling data on the suction surface (Table 3.1 and Smith, et al., 2000) is compared to the high turbulence, suction surface film cooling data in Table 3.10, the result would be a ratio $\left(\mathrm{h}_{\mathrm{t}, \mathrm{c}} / \mathrm{h}_{\mathrm{o}}\right)$ of approximately 1.16-1.34. Johnston, et al. (1999) reported data of this type for heat transfer data with film cooling and high freestream turbulence on a quarter cylinder model. They reported ratios as high as $1.6 \mathrm{on}$ areas with curvature, which is significantly higher than the predicted data in the current work. Factors such as the film cooling blowing rate, freestream turbulence level and curvature will have a large effect on the results. This is evidenced by the ratios of film 
cooled heat transfer coefficient and baseline heat transfer coefficient $\left(h_{o, c} / h_{o}\right)$, which in the work of Johnston, et al. (1999) are between 1.3 and 1.5 in areas of curvature, where in Smith, et al. (2000) this ratio is between 1.06 and 1.26.

Table 3.10. Analytical model applied to film-cooled data from Smith, et al. (2000)

\begin{tabular}{|c|c|c|c|c|c|c|}
\hline Gage & $\mathbf{x} / \mathbf{c}_{\mathbf{p s}}$ & $\begin{array}{c}\Delta \mathbf{h}_{\mathbf{t}} \\
\left(\mathbf{W} / \mathbf{m}^{2} \mathbf{K}\right)\end{array}$ & $\begin{array}{c}\mathbf{h}_{\mathbf{o , c}} \\
\left(\mathbf{W} / \mathbf{m}^{2} \mathbf{K}\right)\end{array}$ & $\begin{array}{c}\text { Predicted } \\
\mathbf{T} \text { urbulent } \\
\mathbf{h}_{\mathbf{t , c}} \\
\left(\mathbf{W} / \mathbf{m}^{\mathbf{2}} \mathbf{K}\right)\end{array}$ & $\mathbf{h}_{\mathbf{t , c}} / \mathbf{h}_{\mathbf{o}, \mathbf{c}}$ & $\mathbf{h}_{\mathbf{t , c}} / \mathbf{h}_{\mathbf{o}}$ \\
\hline SS1 & 0.16 & 59 & 864 & 923 & 1.07 & 1.34 \\
\hline SS2 & 0.20 & 59 & 785 & 844 & 1.08 & 1.20 \\
\hline SS3 & 0.24 & 59 & 622 & 681 & 1.10 & 1.16 \\
\hline SS4 & 0.28 & 59 & 783 & 842 & 1.08 & 1.16 \\
\hline SS5 & 0.32 & 59 & 747 & 806 & 1.08 & 1.16 \\
\hline SS6 & 0.36 & 59 & 875 & 934 & 1.07 & 1.27 \\
\hline
\end{tabular}

\subsubsection{Comparison with Other Correlations}

The existing correlations in the open literature typically correlate normalized heat transfer coefficients $(\mathrm{Nu}, \mathrm{St})$ with integral parameters $\left(\mathrm{Tu}, \Lambda_{\mathrm{x}}\right)$. Several of the existing correlations, discussed in section 1.2.1, were applied to the data in the current work. Many of these correlations require information on boundary layer and momentum thickness, values that have not been determined for the current work. This limits the correlations that could be applied for comparison. The following sections present the results of the application of several correlations to the current data.

\subsubsection{Dullenkopf and Mayle (1995) Effective Turbulence Correlation}

Dullenkopf and Mayle (1995) proposed a correlation for the effects of freestream turbulence on laminar heat transfer downstream of a cylinder stagnation point and on the pressure surface of some airfoils (assuming regions of constant freestream strain rate). The correlation uses an "effective" turbulence level and takes into account the strain rate. The Nusselt number was presented as a function of this effective turbulence level by:

$$
N u_{a} \cdot \operatorname{Pr}^{-0.37}=0.571+0.01 \cdot T u_{\lambda}
$$


where the value of $T u_{\lambda}$ is determine by:

$$
\begin{gathered}
a=\text { strain rate }=\frac{d U_{e}}{d x} \\
N u_{a}=\frac{h \cdot \sqrt{v / a}}{k} \\
T u_{a}=\frac{u_{r m s}^{\prime}}{\sqrt{a \cdot v}} \\
L_{a}=\frac{\Lambda_{x}}{\sqrt{v / a}} \\
T u_{\lambda}=\frac{T u_{a} \sqrt{L_{a}}}{\left.1+0.004 \cdot L_{a}^{2}\right]^{\Gamma / 12}}=\frac{u_{e f f}^{\prime}}{\sqrt{a \cdot v}}
\end{gathered}
$$

The strain rate is used to form both a non-dimensional length scale, $\mathrm{L}_{\mathrm{a}}$, and to form $T u_{\lambda}$. The $u_{\text {eff }}^{\prime}$ represents an "effective" turbulence level considering strain rate and turbulent length scale. The correlation was applied to the data for both the pressure and suction surfaces of the transonic turbine blades. The results are presented below in Figure 3.22. The data reflects uncertainty in the measured heat transfer coefficient of $\pm 6.3 \%$ and $\pm 5.5 \%$ on turbulence intensity. The data from the pressure surface (PS1 and PS2) are in fairly good agreement with the Dullenkopf and Mayle (1995) correlation, while the data for the suction surface deviates from the correlation. This is most likely due to the fact that the suction surface location is in a region of highly accelerating flow, where the freestream strain rate is changing dramatically, while the pressure surface locations are in a region of relatively constant strain rate (as evidenced by the Mach number distribution shown earlier in Figure 2.4) 


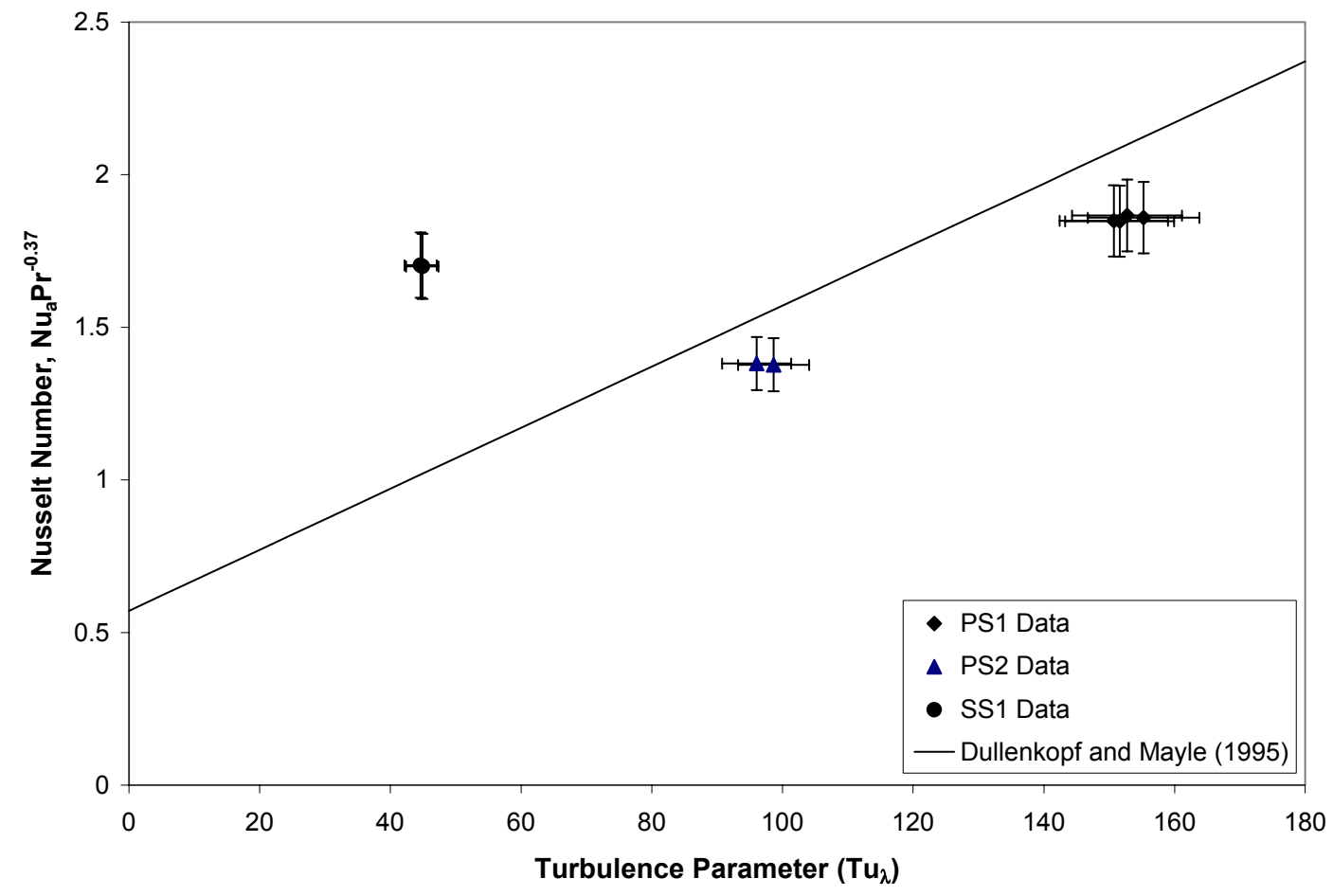

Figure 3.22 Comparison with Dullenkopf and Mayle (1995) effective turbulence correlation

\subsubsection{Maciejewski and Moffat (1992) St' Correlation}

As discussed in section 1.2.1.1, Maciejewski and Moffat (1992) presented a correlation for the effects of high freestream turbulence on flat plate turbulent boundary layer heat transfer data. They proposed a simple method of analyzing high turbulence heat transfer data and observed a linear relationship between measured heat transfer coefficient $(\mathrm{h})$ and turbulent fluctuations in the freestream $\left(\mathrm{u}^{\prime}\right)$. Their correlation was independent of geometry or length scale, and was simply a function of near wall turbulence level:

$$
\frac{h_{t}}{\rho \cdot c \cdot u_{r m s}^{\prime}}=\text { constant }
$$

where $h_{t}$ is the measured turbulent heat transfer coefficient. In their paper, the authors present a plot that shows the quantity $h_{t} / \rho \cdot c \cdot u_{r m s}^{\prime}$ plotted against turbulence intensity in a range up to approximately $60 \%$ turbulence. The value of $h_{t} / \rho \cdot c \cdot u_{r m s}^{\prime}$ is equivalent to the parameter $\mathrm{St}^{\prime}$, where: 


$$
S t^{\prime}=\frac{h_{t}}{\rho \cdot c \cdot u_{r m s}^{\prime}}=\left[0.24+0.12 \cdot \exp -[(T u-0.11) / 0.055]^{2}\right] \cdot f(\operatorname{Pr})
$$

The correlation was applied to the current data and the results are plotted in Figure 3.23. The figure plots the current data along with the correlation with $\pm 15 \%$ uncertainty curves and uncertainty of $\pm 6.3 \%$ on the measured heat transfer coefficient and $\pm 5.5 \%$ on turbulence intensity

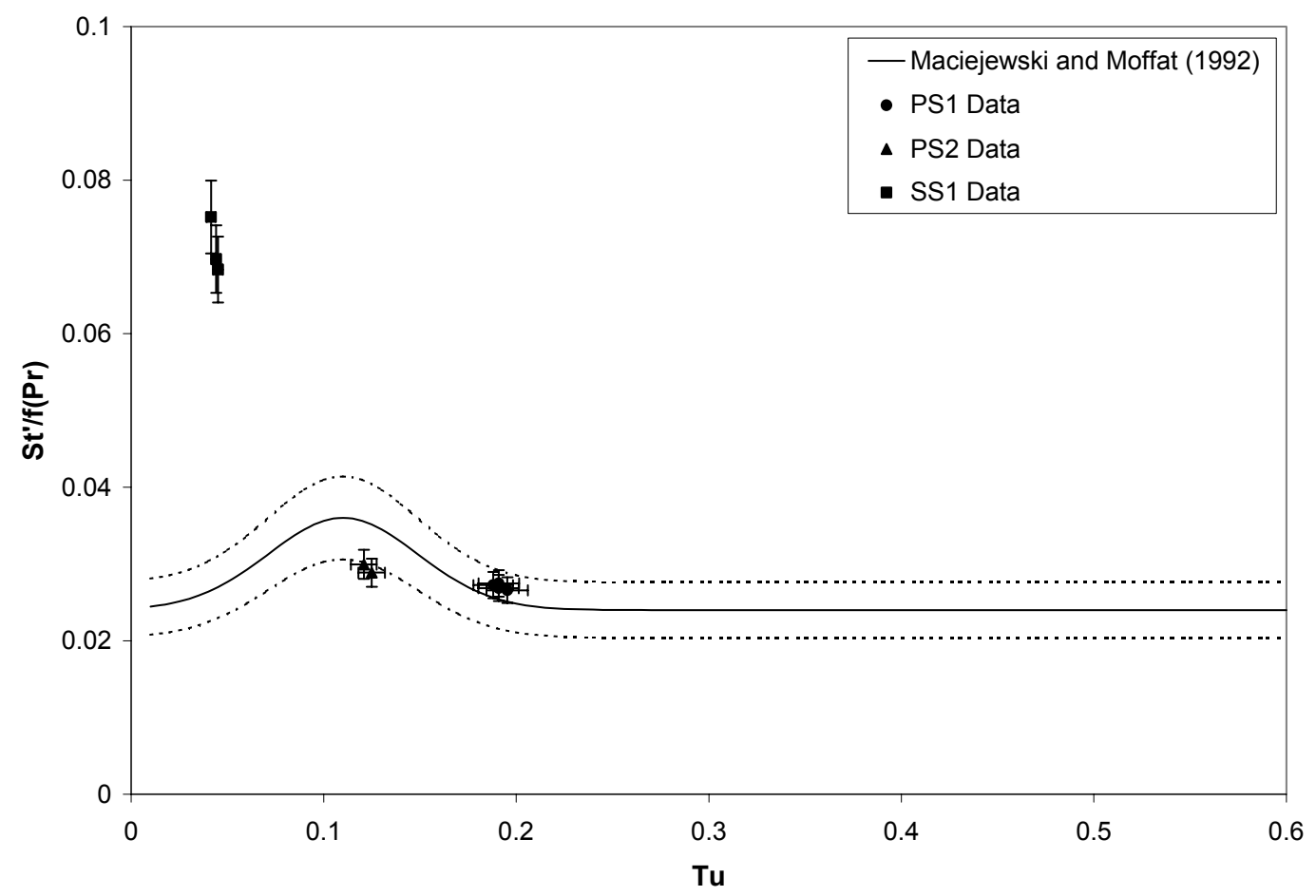

Figure 3.23 Comparison with Maciejewski and Moffat St' correlation

From the figure, it is demonstrated that the correlation matches the measured data well for the pressure surface locations, but the suction surface data $\mathrm{St}^{\prime}$ results are much higher than the correlation predicts. This is similar to the result observed for the Dullenkopf and Mayle (1995) correlation. 


\subsubsection{Van Fossen, Simoneau and Ching (1995) Frossling Number Correlation}

Van Fossen, et. al (1995) studied the influence of turbulence on heat transfer on cylinders and cylinders with wedge shaped afterbodies. A correlation was developed which included the integral length scale in the form of the ratio of the length scale to cylinder leading edge diameter $\left(\Lambda_{\mathrm{x}} / \mathrm{d}\right)$. The correlation is independent of body shape (other than cylinder diameter) and presented a correlation in terms of the Frossling number augmentation as:

$$
\frac{\operatorname{Fr}(0)_{\text {turb }}}{\operatorname{Fr}(0)_{\text {lam }}}=0.00851 \sqrt{\operatorname{Tu} \cdot \operatorname{Re}_{d}^{0.8}\left(\frac{\Lambda_{x}}{d}\right)^{-0.574}}+1
$$

where $\mathrm{d}$ is the leading edge diameter and $\mathrm{Re}$ and $\mathrm{Nu}(\mathrm{Fr}=\mathrm{Nu} / \sqrt{\mathrm{Re}})$ are based on leading edge diameter and inlet velocity.

The correlation was applied to the current data using the leading edge diameter (approximately $0.8 \mathrm{~cm}$ ) and inlet velocity $(140 \mathrm{~m} / \mathrm{s})$, turbulence intensity $(10 \%)$ and length scale $(2 \mathrm{~cm})$. Unlike the other correlations that used local turbulence measurements, the correlation of Van Fossen, et al. (1995) uses turbulence parameters upstream of the body (turbine blade in this case). The results are shown in Figure 3.24 below, including error curves of $\pm 10 \%$ on the correlation and $\pm 2.4 \%$ on the ratio of the Frossling number for the measured data. The correlation fits the data for the pressure surface locations extremely well. The data for both PS1 and PS2 fall on top of each other on the curve. Similar to the results from other correlations presented previously, the suction surface data (SS1) does not match the correlation as well, however, the data do fall within the $\pm 10 \%$ uncertainty. 


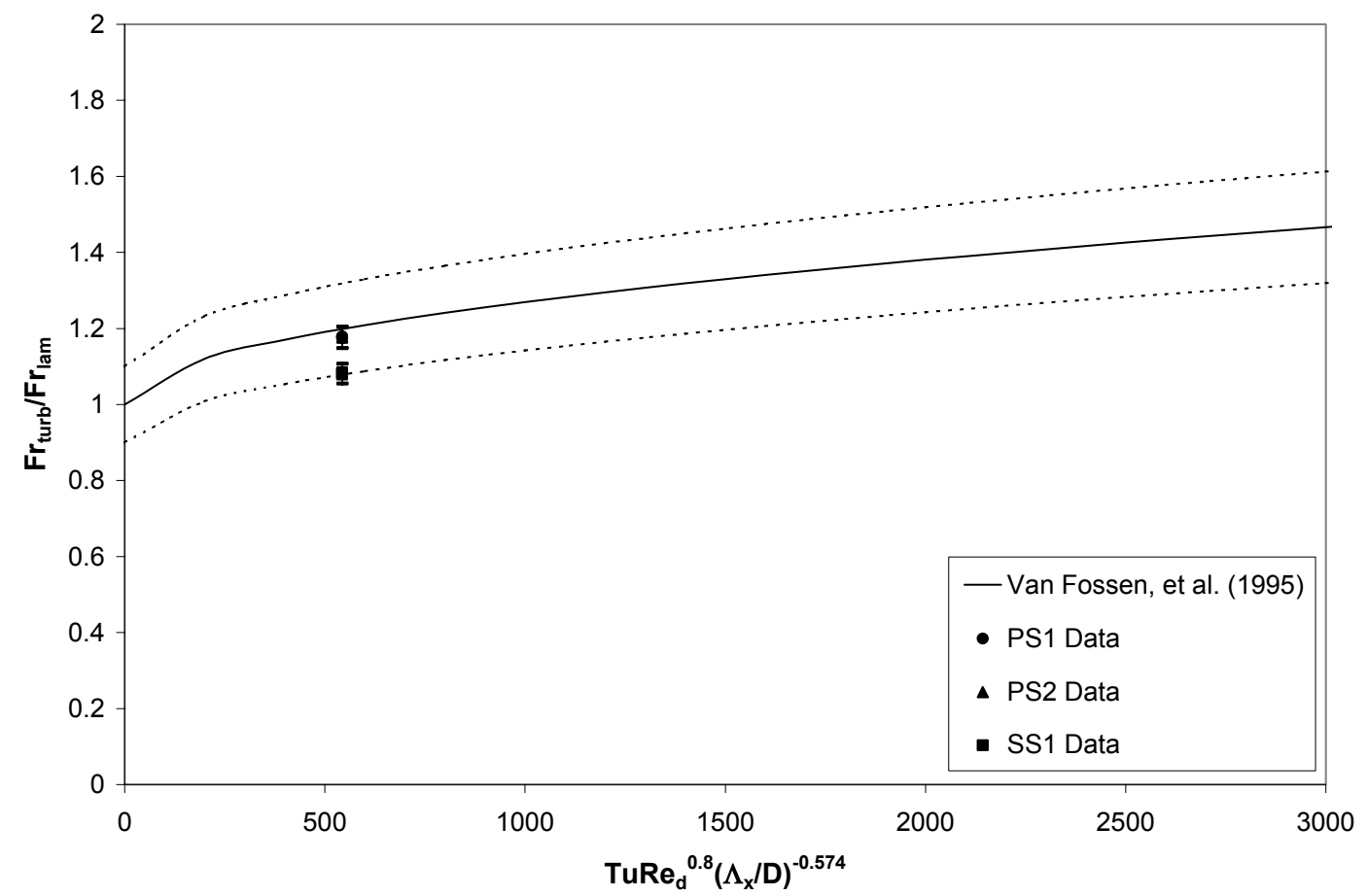

\section{Figure 3.24 Comparison with Van Fossen, et al. (1995) Frossling number correlation}

\subsubsection{Multiple-scale Model}

The single-scale model results presented earlier demonstrated that the analytical model developed shows promise in predicting increases in heat transfer due to turbulence. The single-scale model assumes that all of the turbulent energy in the flow is packed into one representative sized eddy (the integral scale). This is an idealization of the actual flow physics, since in actuality each eddy size has energy associated with it as demonstrated by the power spectrum of the fluctuating velocity. Using the time-resolved signal of fluctuating velocity, it is possible to determine the energy content in the turbulent flowfield for each individual eddy size (i.e. at each frequency). Using this information, it may be possible to predict the increase in heat transfer that is associated with each frequency or eddy size of freestream velocity fluctuations. The model analysis in this form is done in the frequency domain and is referred to in this text as the "multiple-scale" model. The following sections develop the multiple-scale model and analyze its applicability and present some key results. The results of the multiple-scale 
model analysis demonstrate the frequencies of turbulent eddies which have the largest effect on heat transfer. The application of the multiple-scale model to the current data set is analyzed in various forms and the results demonstrate that the model, coupled with the coherence function between unsteady velocity and heat flux gives reasonable predictions of the measured heat transfer increase, although the model results are 3 times higher than the measured values. The analysis presents some interesting points and attempts to determine the range of frequencies, centered on the integral length scale, which will give accurate predictions of the measured increases.

\subsubsection{Development of Multiple-scale Model}

The multiple-scale model basis is similar to the single-scale model with the exception that individual values of the fluctuating energy and length scale at each frequency are used as input into the model to come up with a "spectrum" of increase in heat transfer versus frequency $\left(\Delta \mathrm{q}_{\mathrm{t}}(\mathrm{f})\right)$. The model equation in the multiple-scale form presented as:

$$
\Delta q_{t}(f)=\frac{k \cdot \Delta T}{\left[\pi \alpha \frac{\Lambda_{x}(f)}{u^{\prime}(f)}\right]^{1 / 2}}
$$

In order to apply this equation in the frequency domain, expressions for intensity and length scale of turbulent fluctuations as a function of frequency are needed. From section 3.1.2.4, the expression for the integral length scale was used:

$$
\Lambda_{x}(f)=\frac{3 \cdot \bar{U}}{8 \pi f}
$$

For the turbulent energy at each frequency, the magnitude of the fluctuating component of velocity was determined in the frequency domain. The time-resolved fluctuating velocity signal $\left(\mathrm{u}^{\prime}(\mathrm{t})\right)$ can be transformed into the frequency domain by performing the Fourier Transform (FFT) of the time signal: 


$$
U f=F F T\left(u^{\prime}\right)
$$

The FFT was performed using the FFT function in Matlab software code. The results of the FFT give the real and imaginary components of the fluctuating component of velocity at each frequency. The magnitude of Uf was then determined by:

$$
U f_{\text {mag }}(f)=\sqrt{\operatorname{Imag}(U f(f))^{2}+\operatorname{Real}(U f(f))^{2}}
$$

This magnitude is related to the PSD of fluctuating velocity presented in section 3.1.2.2 by:

$$
\operatorname{PSD}\left(u^{\prime}\right)=\frac{2 \cdot U f_{\text {mag }}(f)^{2}}{\Delta f}
$$

since $\operatorname{Imag}(U f)^{2}+\operatorname{Real}(U f)^{2}=F F T\left(u^{\prime}\right) \cdot F F T^{*}\left(u^{\prime}\right)$. Thus, if we want to determine $u_{R M S}^{\prime}$ from the magnitude of $u^{\prime}$ in the frequency domain $\left(\mathrm{Uf}_{\mathrm{mag}}\right)$, the equation is then:

$$
u_{R M S}^{\prime}=\left[\sum_{f} 2 \cdot U f_{m a g}(f)^{2}\right]^{0.5}
$$

Equation 3.40 was then used to determine $\Lambda_{\mathrm{x}}(\mathrm{f})$ and Equation 3.42 was used to determine the input of $u^{\prime}(f)$ (where the magnitude of $U f(f)$ is used). These values could then be applied in the model to yield a spectrum of increase in heat flux (as presented in Equation 3.39 and in Equation 3.45 below):

$$
\Delta q_{t}(f)=\frac{k \cdot \Delta T}{\left[\pi \alpha \frac{\Lambda_{x}(f)}{u^{\prime}(f)}\right]^{1 / 2}}
$$

$$
\text { where } \Lambda_{x}(f)=\frac{3 \cdot \bar{U}}{8 \pi f} \text { and } u^{\prime}(f)=U f_{\text {mag }}(f)
$$




\subsubsection{Application of Multiple-scale Model}

Equation 3.45 was applied to the data for the pressure surface location nearest the leading edge (PS1) in the current data set in order to test and continue development of the model. Locations PS2 and SS1 will be discussed later. The intensity and length scale of turbulent fluctuations were determined as a function of frequency as input into the multiple-scale model and the resultant increase in heat flux was plotted as a function of frequency. The model predicted data are shown in Figure 3.25 below.

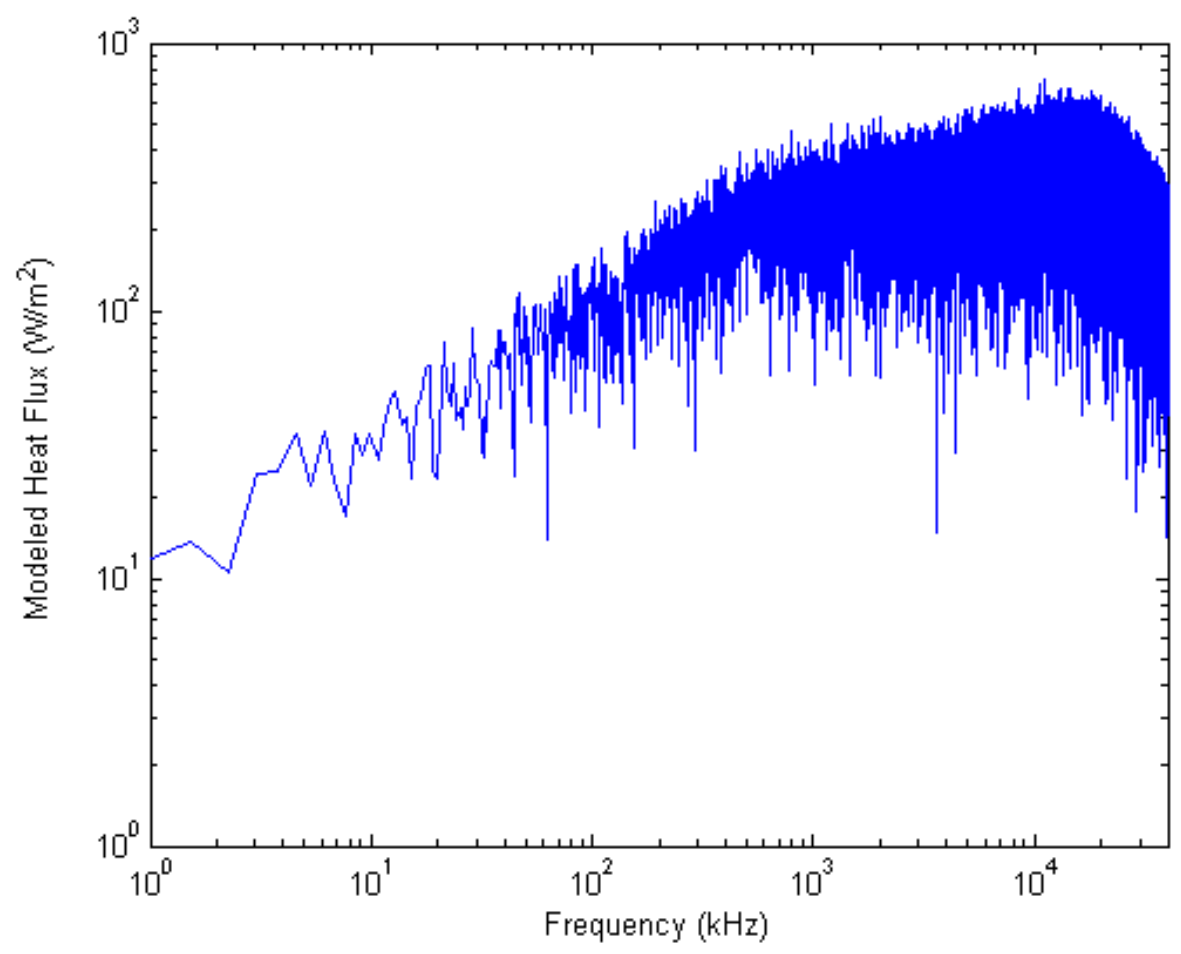

Figure 3.25: Model predicted heat flux increase as a function of frequency

From the figure, it is apparent that the multiple-scale model grossly over predicts the heat transfer at high frequency. The spectrum of heat flux in Figure 3.13 demonstrated that the energy in the unsteady heat flux signal is attenuated at higher frequencies, thus it is expected that the heat flux increases at higher frequencies would also be attenuated. The breakdown in the model may be a direct result of one of the primary assumptions of the model, that the scale of the turbulence is much larger than the boundary layer thickness. For a boundary layer thickness of approximately $1 \mathrm{~mm}$, this would correspond to eddy scales with a frequency of approximately $10-15 \mathrm{kHz}$. If we 
assume "much larger" to be approximately 2-3 times the boundary layer thickness, this corresponds to eddy scales of 5-10 kHz. The model would then break down above these frequencies. Mayle, et al. (1998) state that the frequency of eddies most effective at increasing laminar heat transfer is about $1.3 U_{\infty} / 2 \pi \Lambda_{x}$. For the data in this work, this frequency is approximately $1 \mathrm{kHz}$. The coherence presented in Figure 3.15 earlier demonstrated high coherence in this range for all three gage locations, and the frequency of the integral length scale ranged from approximately $550-700 \mathrm{~Hz}$.

Figure 3.26 plots the model predicted heat flux increases versus the measured heat flux fluctuations $\left(\mathrm{Qf}_{\mathrm{mag}}\right)$ for comparison. The figure again demonstrates that the model predicts much more energy (higher increases in heat flux) at higher frequency than the measured heat flux. The measured fluctuations $\left(\mathrm{Qf}_{\mathrm{mag}}\right)$ are of similar shape as the

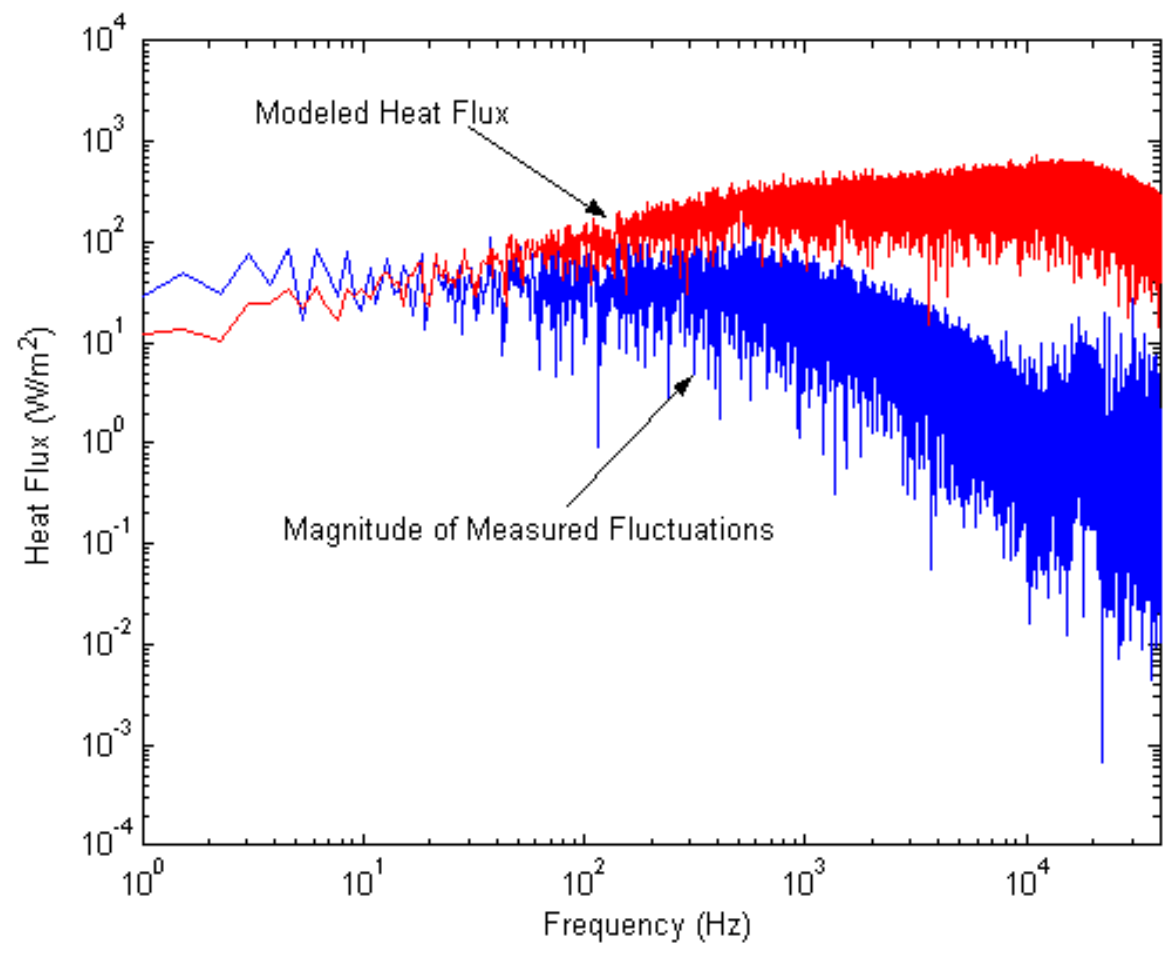

Figure 3.26: Comparison of model predicted heat flux increases with measured fluctuations

measured heat flux PSD (Figure 3.13), as expected since the two are related (similar to the velocity fluctuations) by: 


$$
q_{P S D}^{\prime}=\frac{2 \cdot Q f_{m a g}(f)^{2}}{\Delta f}
$$

Two key points arise from these results. First, what can be done (or more appropriately what is missing from the model) to qualitatively predict the correct trend in the heat flux increases in the frequency domain. The model predicts large increases in heat transfer due to high frequency velocity fluctuations while the PSD of heat flux and the coherence disagree with these predictions. The PSD of measured heat flux shows very low energy at high frequency, and the coherence function indicates little to no correlation between the fluctuations in velocity and heat flux at frequencies above $5 \mathrm{kHz}$ for the current data. Second, how can the predicted heat flux at each frequency be combined to obtain similar results to the single-scale model (i.e. how do we combine the data in the frequency domain to determine the representative heat flux event)? The second point will be addressed in the next section.

In work by Dullenkopf and Mayle (1995) and Mayle, et al. (1998), the idea of an "effective" turbulence level was introduced. Their ideas were developed based on stability theory for a laminar boundary layer. Fluctuations at high frequencies (small scale) are viscously damped, while disturbances at low frequency (large scale) are seen as quasi-steady, similar to bulk flow pulsations, which in the mean do not affect heat transfer. Only a small frequency band around a dominant frequency is effective at increasing heat transfer. This would indicate that the model predicted increases at high frequency would not exist. The ability of freestream turbulence to affect surface heat transfer can be determined by observation of the coherence between the time-resolved velocity and heat flux signals. The coherence for each of the three gage locations in this work was presented in Figure 3.15 in section 3.3.2.2. From the figure (shown again below as Figure 3.27) it is apparent that the effect of turbulent fluctuations on surface heat transfer is almost completely attenuated at frequencies above $5 \mathrm{kHz}$.

Due to the resolution of the data at low frequency, it is difficult to obtain accurate information from the spectra and coherence functions at low frequency. The coherence function does, however, agree with the ideas presented by Dullenkopf and Mayle where high frequency fluctuations do not affect heat transfer and coherence between velocity 
and heat flux is only present in a narrow frequency range around the integral length scale (as previously discussed). The dashed lines on the coherence plot represent the range of the frequencies of the integral length scales measured at the three blade locations with an uncertainty band of $\pm 5 \%$ applied. The logical step is then to make use of the knowledge gained from the time-resolved measurements of velocity and heat flux in the form of the coherence function and incorporate the coherence into the multiple-scale model. Since the coherence is a dimensionless function, it was decided to perform a direct multiplication of the coherence function with the model output, coupling the two data sets in the frequency domain.

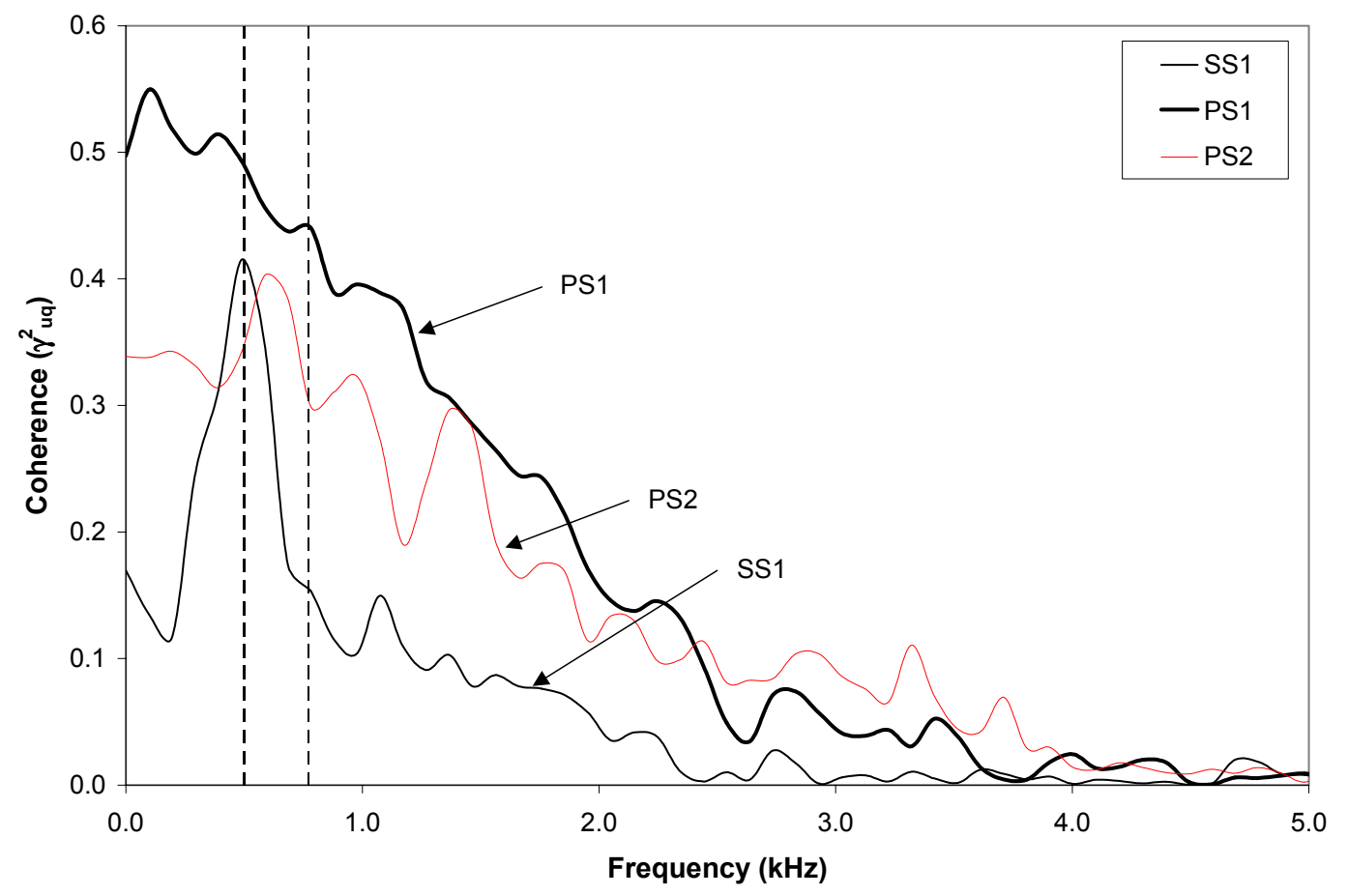

Figure 3.27: Coherence between velocity and heat flux signals

The resultant revised model equation is then:

$$
\Delta q_{t}(f)=\frac{k \cdot \Delta T}{\left[\pi \alpha \frac{\Lambda_{x}(f)}{u^{\prime}(f)}\right]^{1 / 2}} \cdot \gamma_{u q}^{2}(f)
$$


where the value $\gamma_{u q}^{2}(f)$ is the measured coherence function between the time-resolved velocity and heat flux data as a function of frequency. This value is then equal to the coherent output power (COP) or the energy resultant in the output signal (surface heat flux) that is correlated with the input signal (freestream velocity fluctuations). The resultant model predicted data are shown in Figure 3.28a.

From the figure, it is shown that the model predicted data follows the same general trend as the measured heat flux spectra, although there is some disagreement in the magnitude of the heat flux. This difference in magnitude is further illustrated in Figure 3.28b, which presents the same data as Figure 3.28a plotted on a linear scale. The application of the coherence function has effectively attenuated the large predicted increases in heat transfer at higher frequencies, which is expected from the coherence and measured heat flux spectra. Although the predicted heat flux at high frequency is reduced and the spectrum of heat flux increase has a shape that is more consistent with the measured spectra, the question of how to use this data to find a total increase in heat transfer still remains.

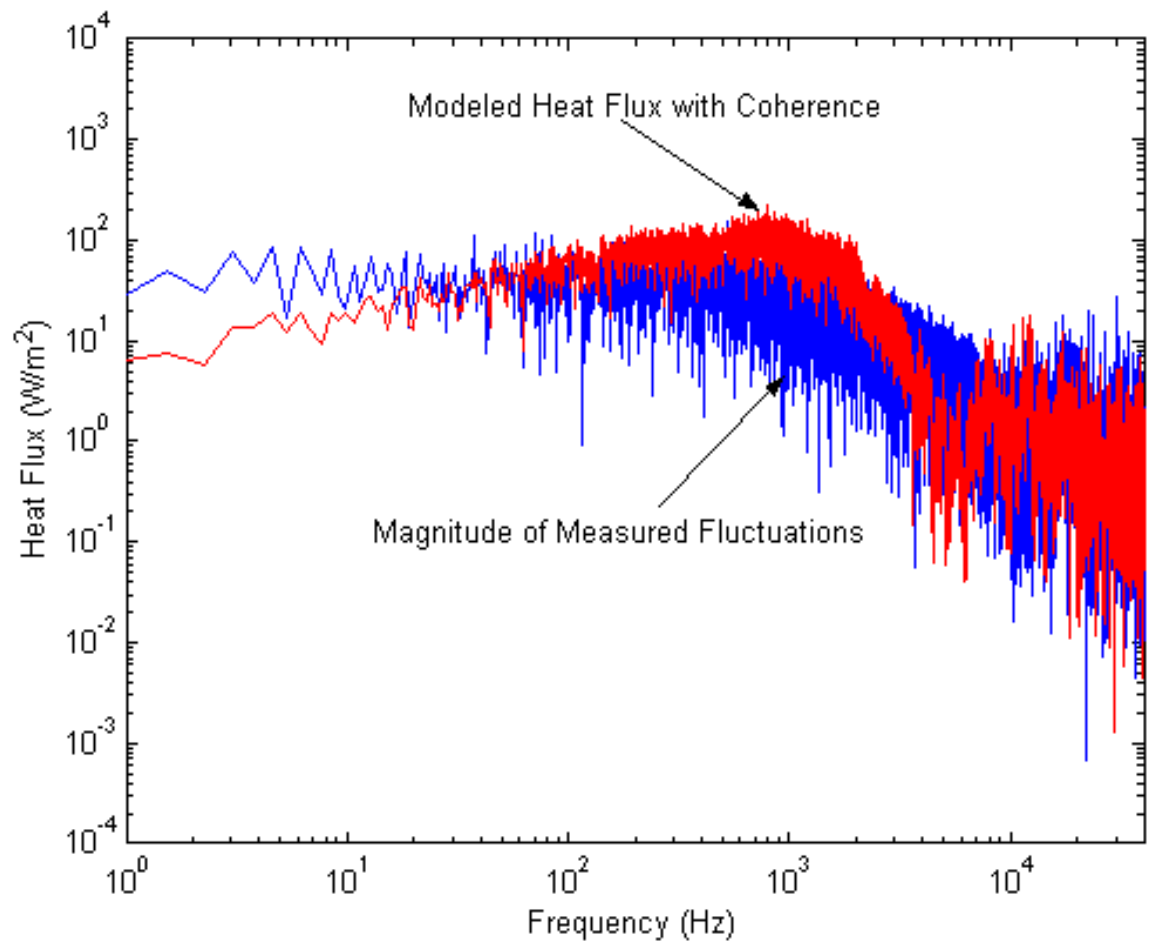

Figure 3.28a: Predicted model heat flux with coherence applied and measured heat flux fluctuations 


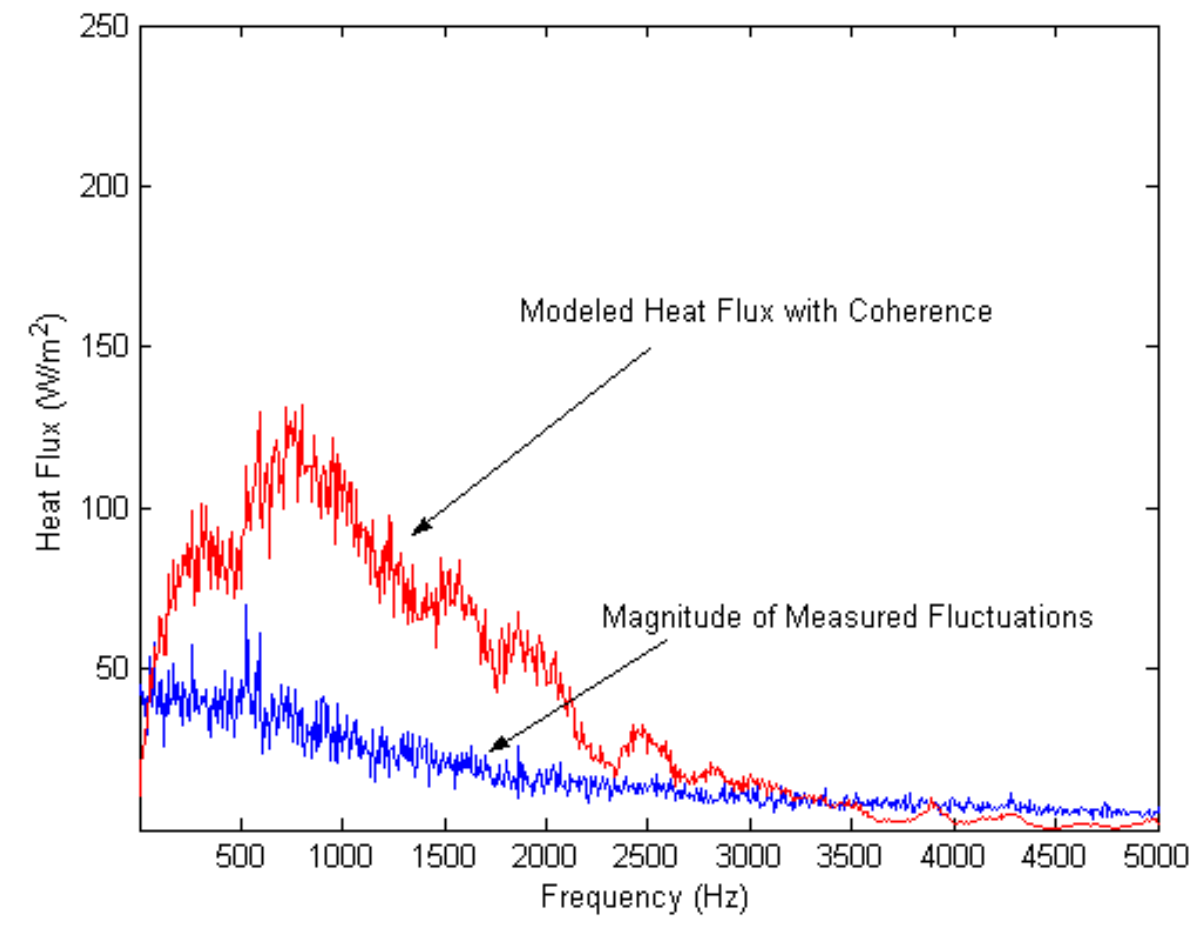

\section{Figure 3.28b: Predicted model heat flux with coherence applied and measured heat flux fluctuations (Linear scale)}

The second key point discussed earlier is then important, how can the data be combined in the frequency domain to yield a total increase in heat transfer? Is the total increase in heat flux equal to the sum of the predicted increase at all frequencies? From a physical standpoint, summation across all frequencies would indicate that each individual eddy acts independently of eddies at other frequencies to increase surface heat transfer. The next step is then to determine how to combine the heat flux increases for the frequency domain modeled data to give a value of predicted heat flux. The following section investigates several different methods for combining the data.

\subsubsection{Combination of Multiple-scale Model Data}

In the single-scale model, the increase in heat transfer is partially governed (along with the length scale) by the root mean square (RMS) of the velocity fluctuations, rather than treating each eddy size with its associated energy independently. The RMS of the fluctuating energy is packed into one representative eddy size in the single-scale model. This concept may be applied to the summation of the predicted heat flux increases, which 
inherently assumes that if one eddy of smaller size (higher frequency) is resident at the surface with a larger (lower frequency) eddy, the two do not act independently of one another to increase heat transfer, rather the two eddies combine energy in a non-linear fashion. Graphically, the combined increases in heat flux can be represented in the time domain by Figure 3.29.

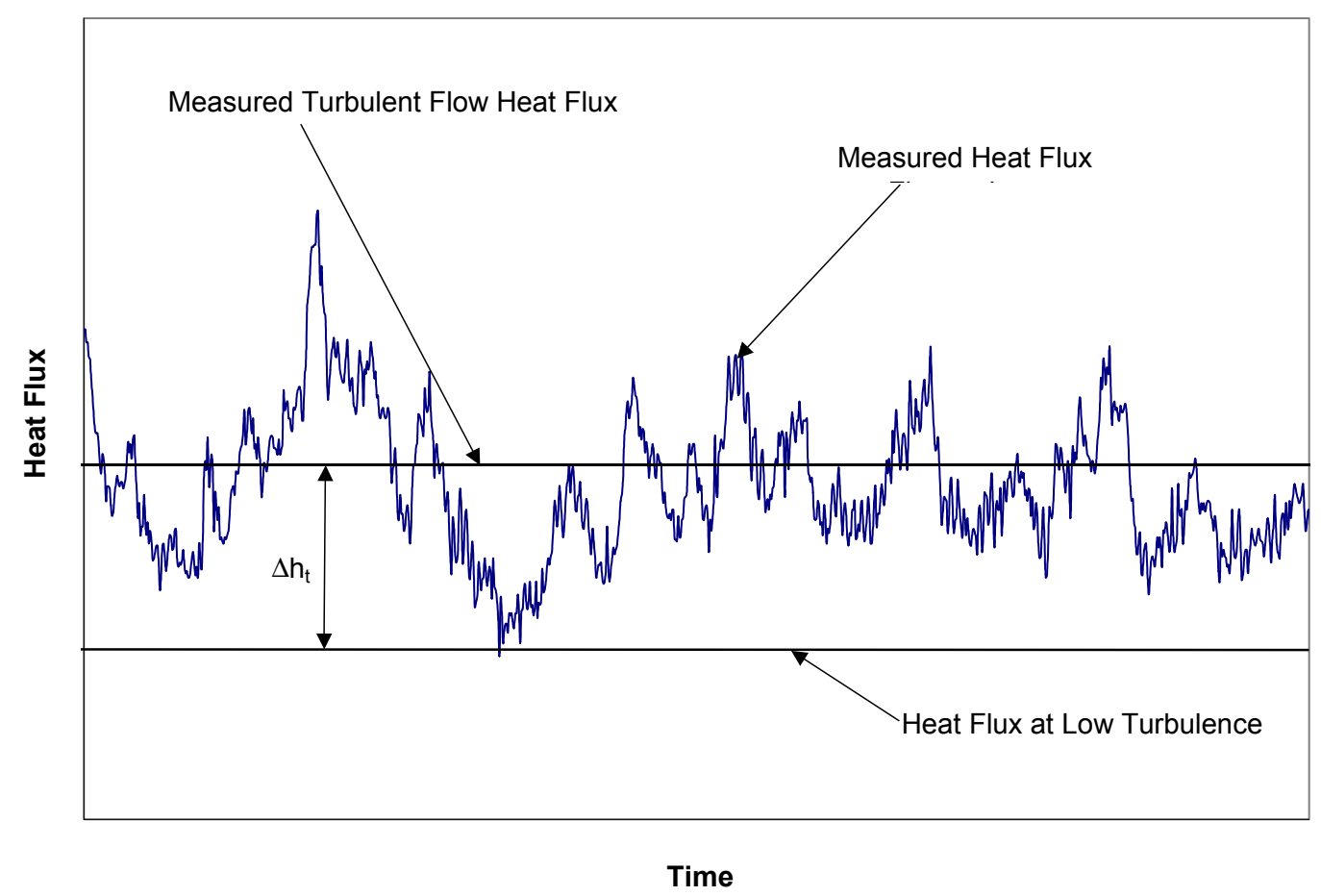

Figure 3.29: Illustration of increase in heat transfer by turbulent fluctuations in heat flux

The figure demonstrates the idea that the fluctuations in heat transfer can be added to the heat flux without freestream turbulence, assuming that even eddies moving away from the surface (negative velocity fluctuations) cause an increase in heat transfer. The value of $\Delta \mathrm{h}_{\mathrm{t}}$ in the figure would then be equal to the RMS of the fluctuating component of measured heat flux. This also assumes that ALL frequencies of heat flux fluctuations are included in the total increase. As the coherence has demonstrated, only frequencies in the range up to $5 \mathrm{kHz}$ are correlated with velocity fluctuations. The modeled heat flux could then be combined by finding the total heat flux increase by using an equation of similar form as was used for determining RMS of velocity. This 
essentially characterizes a representative heat flux event, similar to how the RMS of fluctuating velocity characterizes turbulence.

The combination of the heat flux increases for the multiple-scale model was investigated in various forms in an attempt to determine how to apply the multiple-scale model and to investigate which frequencies have the dominant effect on surface heat transfer. The methods used to combine the heat flux increases are discussed below along with an illustration of the application of the equations to the data for each method. The results of each different method are presented in Table 3.10. The methods can essentially be categorized into three forms. The first (Methods 1 and 2) uses the RMS of the measured time-resolved heat flux data to compare these results to the average measured heat flux increase and single-scale model data (as illustrated in Figure 3.29), the second (Methods 3 and 4) uses the multiple-scale model developed in the previous section and the third (Methods 6 and 7) is a variation on the single-scale model, using the frequency domain velocity data to determine the ratio of $U f_{m a g}(f)$ and $\Lambda_{x}(f)$ from equation 3.45 and finding the RMS of this data. This method is essentially a hybrid of the single-scale and multiple-scale models, applying concepts developed for the multiple-scale model to the velocity and length scale (determining each in the frequency domain) then entering an RMS value of the ratio between these two parameters into the single-scale model. For each of these three methods, the results are presented with and without the coherence function applied. In addition, the total increase in heat transfer was determined using the whole frequency range as well as with the data combined only over the frequency range of significant coherence $(0-5 \mathrm{kHz})$. The single-scale model is presented for comparison as Method 5. 
Methods investigated for combining heat flux increases in frequency domain:

1. Using measured $\mathrm{Qf}_{\mathrm{mag}}$, determine $\Delta \mathrm{q}_{\mathrm{t}, \text { total }}$ using $\mathrm{RMS}$ equation for all frequencies and for data from $0-5 \mathrm{kHz}$ :

Across all frequencies:

$$
\Delta q_{t, \text { total }}=\sqrt{\sum_{f} 2 \cdot Q f_{\text {mag }}(f)^{2}}
$$

From $0-5 \mathrm{kHz}$ (range of coherence):

$$
\Delta q_{t, \text { total }(0-5 \mathrm{kHz})}=\sqrt{\sum_{f=0-5 \mathrm{kHz}} 2 \cdot Q f_{\text {mag }}(f)^{2}}
$$

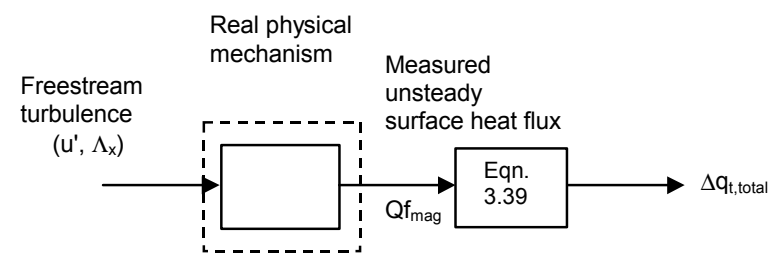

2. Using measured $\mathrm{Qf}_{\mathrm{mag}}$ multiplied by the coherence $\left(\gamma_{\mathrm{uq}}{ }^{2}\right)$, determine $\Delta \mathrm{q}_{\mathrm{t}, \text { total }}$ using RMS equation for all frequencies and for data from 0-5 $\mathrm{kHz}$ :

Across all frequencies:

$$
\Delta q_{t, \text { total }}=\sqrt{\sum_{f} 2 \cdot\left(Q f_{\text {mag }}(f) \cdot \gamma_{u q}^{2}(f)\right)^{2}}
$$

From $0-5 \mathrm{kHz}$ (range of coherence):

$$
\Delta q_{t, \text { total }(0-5 \mathrm{kHz})}=\sqrt{\sum_{f=0-5 \mathrm{kHz}} 2 \cdot\left(Q f_{m a g}(f) \cdot \gamma_{u q}^{2}(f)\right)^{2}}
$$

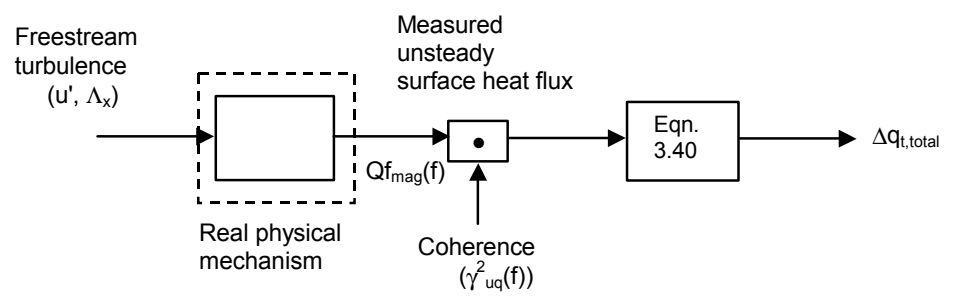


3. Using multiple-scale modeled $\Delta \mathrm{q}_{\mathrm{t}}(\mathrm{f})$, determine $\Delta \mathrm{q}_{\mathrm{t}, \text { total }}$ using RMS equation for all frequencies and for data from $0-5 \mathrm{kHz}$ :

Across all frequencies:

$$
\Delta q_{t, \text { total }}=\sqrt{\sum_{f} 2 \cdot \Delta q_{t}(f)^{2}}
$$

From $0-5 \mathrm{kHz}$ (range of coherence):

$$
\Delta q_{t, \text { total }(0-5 \mathrm{kHz})}=\sqrt{\sum_{f=0-5 k H z} 2 \cdot \Delta q_{t}(f)^{2}}
$$

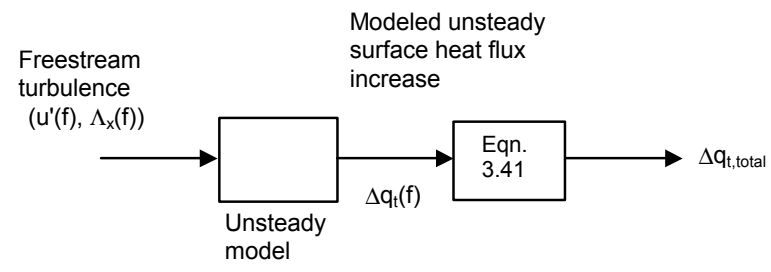

4. Using multiple-scale modeled $\Delta \mathrm{q}_{\mathrm{t}}(\mathrm{f})$ multiplied by the coherence $\left(\gamma_{\mathrm{uq}}{ }^{2}\right)$, determine $\Delta \mathrm{q}_{\mathrm{t}, \text { total }}$ using RMS equation for all frequencies and for data from 0 $5 \mathrm{kHz}$ :

Across all frequencies:

$$
\Delta q_{t, \text { total }}=\sqrt{\sum_{f} 2 \cdot\left(\Delta q_{t}(f) \cdot \gamma_{u q}^{2}(f)\right)^{2}}
$$

From $0-5 \mathrm{kHz}$ (range of coherence):

$$
\Delta q_{t, \text { total }(0-5 \mathrm{kHz})}=\sqrt{\sum_{f=0-5 \mathrm{kHz}} 2 \cdot\left(\Delta q_{t}(f) \cdot \gamma_{u q}^{2}(f)\right)^{2}}
$$

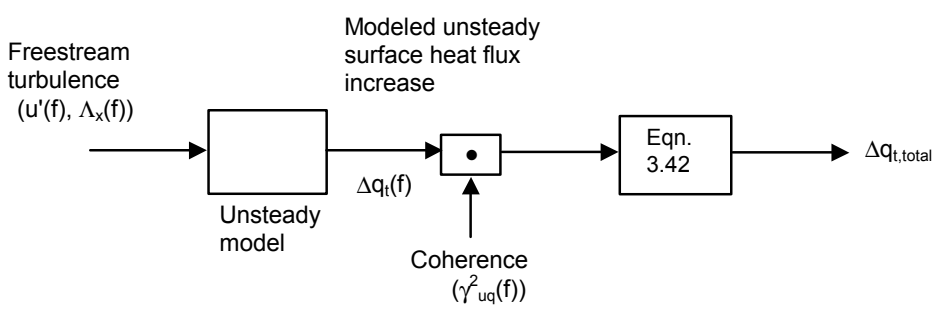


5. Using measured $\mathrm{u}_{\mathrm{rms}}^{\prime}$ and $\Lambda_{\mathrm{x}}$ (equivalent to single-scale model) across all frequencies:

$$
\begin{gathered}
u_{R M S}^{\prime}=\sqrt{\sum_{f} P S D_{u}(f) \cdot \Delta f}=\sqrt{\overline{u^{\prime}(t)^{2}}} \\
\Delta q_{t, \text { total }}=\frac{k \cdot \Delta T}{\left[\pi \alpha \frac{\Lambda_{x}}{u_{R M S}^{\prime}}\right]^{1 / 2}}
\end{gathered}
$$

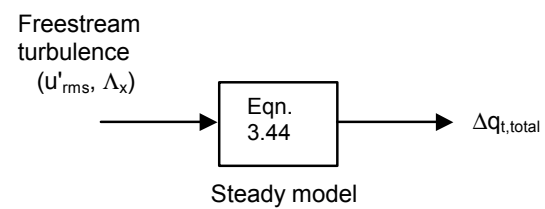

6. Using RMS of $\mathrm{u}^{\prime} / \Lambda_{\mathrm{x}}$ including data across all frequencies and from $0-5 \mathrm{kHz}$ :

Across all frequencies:

$$
\left(\frac{u^{\prime}}{\Lambda_{x}}\right)_{R M S}=\sqrt{\sum_{f} 2 \cdot\left(\frac{u^{\prime}(f)}{\Lambda_{x}(f)}\right)^{2}}
$$

From $0-5 \mathrm{kHz}$ (range of coherence):

$$
\begin{aligned}
& \left(\frac{u^{\prime}}{\Lambda_{x}}\right)_{R M S}=\sqrt{\sum_{f=0-5 k H z} 2 \cdot\left(\frac{u^{\prime}(f)}{\Lambda_{x}(f)}\right)^{2}} \\
& \Delta q_{t, \text { total }}=\frac{k \cdot \Delta T}{[\pi \alpha]^{1 / 2}}\left(\frac{u^{\prime}}{\Lambda_{x}}\right)_{R M S}^{1 / 2}
\end{aligned}
$$

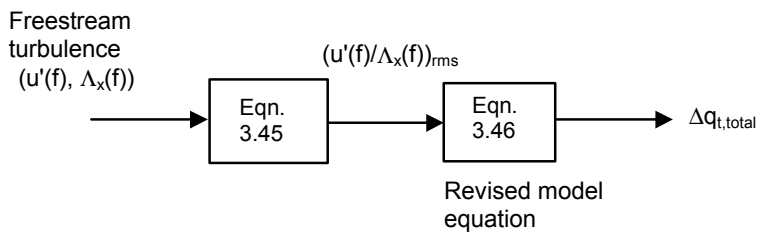


7. Using RMS of $\mathrm{u}^{\prime} / \Lambda_{\mathrm{x}}$ multiplied by the coherence $\left(\gamma_{\mathrm{uq}}{ }^{2}\right)$, including data across all frequencies and from 0-5 kHz:

Across all frequencies:

$$
\left(\frac{u^{\prime}}{\Lambda_{x}}\right)_{R M S}=\sqrt{\sum_{f} 2 \cdot\left(\frac{u^{\prime}(f)}{\Lambda_{x}(f)} \cdot \gamma_{u q}^{2}(f)\right)^{2}}
$$

From $0-5 \mathrm{kHz}$ (range of coherence):

$$
\begin{aligned}
& \left(\frac{u^{\prime}}{\Lambda_{x}}\right)_{R M S}=\sqrt{\sum_{f=0-5 k H z} 2 \cdot\left(\frac{u^{\prime}(f)}{\Lambda_{x}(f)} \cdot \gamma_{u q}^{2}(f)\right)^{2}} \\
& \Delta q_{t, \text { total }}=\frac{k \cdot \Delta T}{[\pi \alpha]^{1 / 2}}\left(\frac{u^{\prime}}{\Lambda_{x}}\right)_{R M S}^{1 / 2}
\end{aligned}
$$

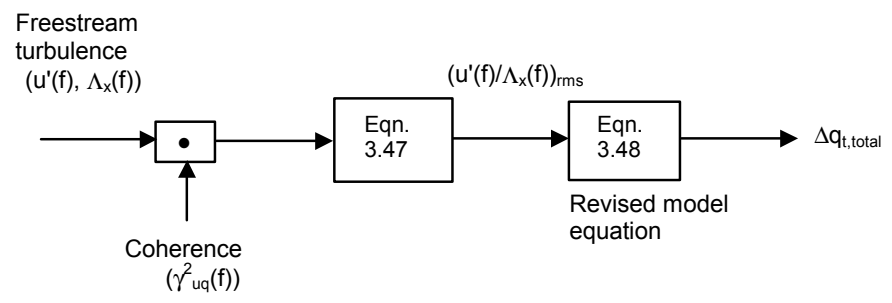

Using each of these methods, the total increase in heat transfer due to turbulence was determined and the results are shown below in Table 3.10. 
Table 3.11: Multiple-scale model results by various methods for location PS1

\begin{tabular}{|c|c|c|}
\hline Method & $\begin{array}{c}\Delta \mathbf{q}_{\text {total }} \\
\left(\mathbf{W} / \mathbf{m}^{\mathbf{2}}\right)\end{array}$ & $\begin{array}{c}\Delta \mathbf{q}_{\text {total }}(\mathbf{0}-\mathbf{5} \mathbf{~ k H z}) \\
\left(\mathbf{W} / \mathbf{m}^{2}\right)\end{array}$ \\
\hline 1 & 2785 & 2698 \\
\hline 2 & 1668 & 1109 \\
\hline 3 & 100,400 & 32,560 \\
\hline 4 & 6513 & 6467 \\
\hline 5 & 6048 & 3283 \\
\hline 6 & 1367 & 1360 \\
\hline 7 & \multicolumn{2}{|c|}{2124} \\
\hline
\end{tabular}

The results from the table were analyzed to determine the method that most closely predicts the measured heat flux increase and the value from the single-scale model (Method 5). From the table, it appears as if the idea presented in Figure 3.29, that the RMS of the fluctuating velocity can be added to the measured low turbulence heat transfer to predict the heat transfer with turbulence, is a reasonable assumption. The value of $\Delta q_{\text {total }}$ determined by this method (Method 1) is very close to the measured and single-scale model predicted increase in heat transfer (Method 5). Applying the coherence to the measured RMS fluctuations (Method 2) decreases the predicted value. Clearly, applying the coherence to measured heat flux data is not necessary, since it is data that is already a function of the coherence between velocity and heat flux based on the real physical mechanism by which turbulence affects surface heat transfer.

The results of Methods 3 and 4 were presented earlier graphically in Figure 3.26 and Figure 3.28, respectively. Figure 3.26 presents the multiple-scale model data without the coherence function applied, which, as discussed earlier, does not take into account the fact that high frequency increases in heat transfer are attenuated. The results in Table 3.10 demonstrate that finding the value of $\Delta \mathrm{q}_{\mathrm{t}, \text { total }}$ for this data gives extremely high predictions of the increase in heat transfer. The data with the coherence applied (Method 4) predicts increases in heat transfer on the order of the measured increases, but approximately 3 times higher. This method makes the most physical sense and shows promise in predicting heat transfer increases from frequency domain data, and may 
possibly be used to determine the frequency band which is most effective at increasing heat transfer. This method will be revisited shortly.

The results in Table 3.10 for Methods 6 and 7 demonstrate that both methods predict an increase in heat transfer that are on the same order as the measured increase. As discussed earlier, this method is essentially a hybrid of the single-scale and multiplescale models. The method uses the input velocity and length scale to determine the residence time of each individual frequency of fluctuation and uses an equation of the form of the RMS to determine a representative residence time. This residence time is then entered into the model equation. From the values presented for the data out to $5 \mathrm{kHz}$ in the two methods it can be seen that results of these methods bound the measured (and single-scale model) increase in heat transfer.

Revisiting the results presented for Method 4, the frequency range for which data were included was investigated by centering the frequency band on the frequency of the integral length scale. This frequency was determined in section 3.3.2.2 to be $570 \mathrm{~Hz}$ for the data presented above (location PS1 - pressure surface nearest the leading edge). The multiple-scale model results with the coherence applied were combined using equation $3.49 \mathrm{~b}$, but rather than combining data from $0-5 \mathrm{kHz}$, a frequency band spanning a range of $f_{\Lambda}+-\Delta f_{b}$ was used (where $f_{\Lambda}$ is the frequency corresponding to the measured integral length scale $(570 \mathrm{~Hz})$ and $\Delta \mathrm{f}_{\mathrm{b}}$ is the half-width of a frequency band centered on $\left.\mathrm{f}_{\Lambda}\right)$. This value of $\Delta \mathrm{f}_{\mathrm{b}}$ was varied until the total heat flux increase $\left(\Delta \mathrm{q}_{\mathrm{t}, \text { total }}\right)$ was approximately equal to the measured increase in heat transfer. The results of modeling showed that $\Delta \mathrm{f}_{\mathrm{b}}$ was equal to approximately $75 \mathrm{~Hz}$. This result is demonstrated graphically in Figure 3.30 below. In the figure, the modeled heat flux data is identical to that presented in Figure 3.28 except the data has been frequency averaged to reduce the noise in the spectrum for presentation purposes.

The analysis of the model data in this form is similar to the analysis performed in Dullenkopf and Mayle (1995). In that work, it was stated: "The effective turbulence level, $\mathrm{Tu}_{\mathrm{eff}}$, is obtained by integrating the spectral energy density over a band width of frequencies centered about the dominant frequency." The dominant frequency in their case was a dimensionless frequency $\left(\phi_{\lambda}\right)$ based on the frequency of the integral length scale and the frequency band was $\Delta \phi$. Their frequency band is a dimensionless 
equivalent of $2 \Delta \mathrm{f}_{\mathrm{b}}$. The work of Dullenkopf and Mayle applied this concept to the velocity spectra and then used this effective turbulence level in a correlation with the integral length scale to determine increases in heat transfer. Their results showed that the frequency band which is effective at increasing surface heat transfer is much smaller than the dominant frequency $\left(\Delta \phi<<\phi_{\lambda}\right)$. In this work, the heat transfer has been modeled and this concept is applied to the resultant data with similar results. The frequency band that yields the measured increase in heat transfer is small (approximately 1/10 of the integral scale frequency), as illustrated in Figure 3.30.

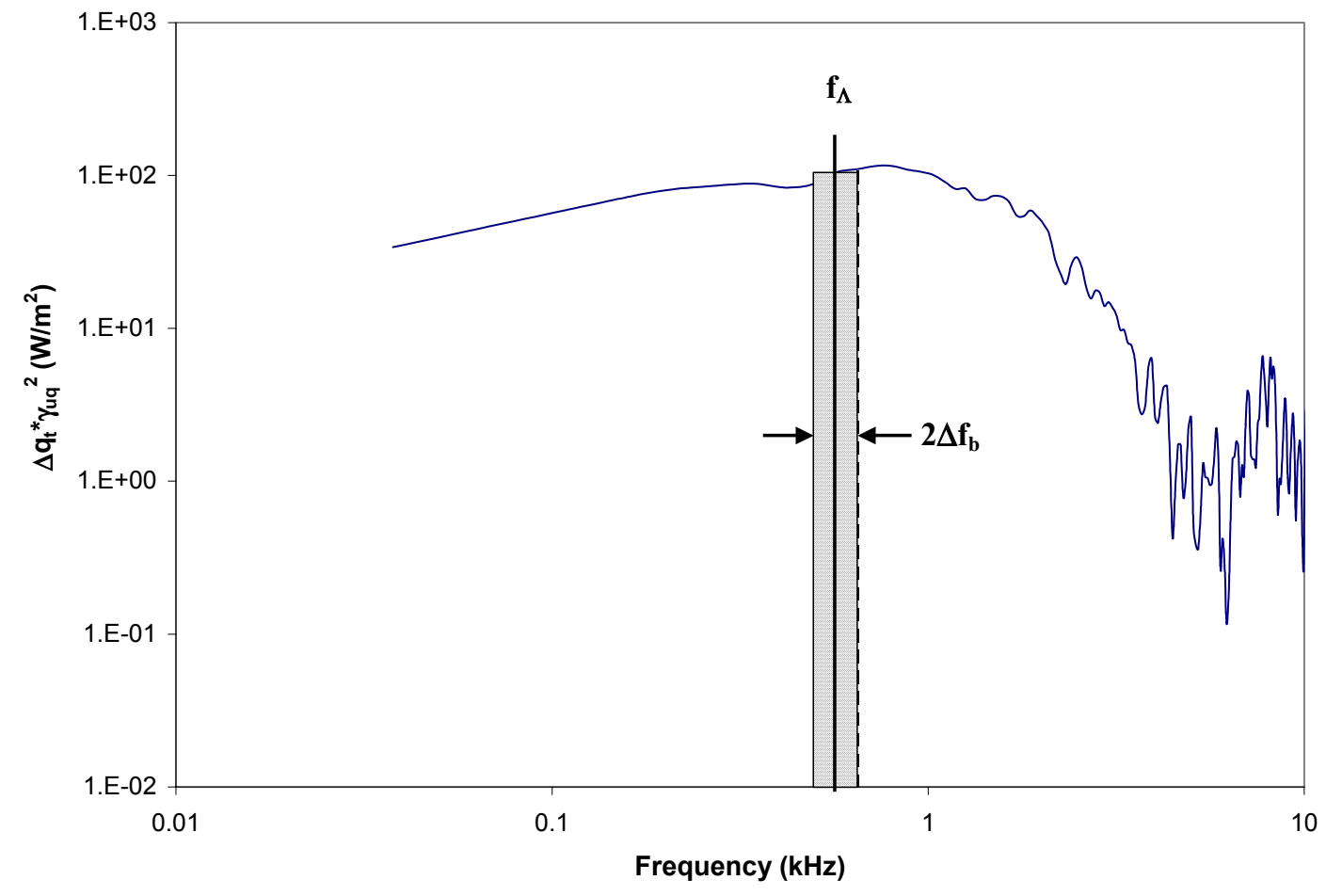

Figure 3.30: Illustration of frequency band of multiple-scale heat flux model

With the data modeled and analyzed for one location in the current data set, the next logical step is to apply the analyses to the other two locations to increase the number of data points for the analysis of the different methods. The methods presented above were applied to the data sets for location PS2 (on the pressure surface of the blade further along the blade chord in the streamwise direction) and for location SS1 (on the suction 
surface of the turbine blade). The results for these two locations are show in Table 3.11 in the same format as the data presented in Table 3.10 for location PS1.

Table 3.12: Multiple-scale model results by various methods for locations PS2 and SS1

\begin{tabular}{|c|c|c|}
\hline Method & $\begin{array}{r}\Delta \mathbf{q}_{\text {total }} \\
\left(\mathbf{W} / \mathbf{m}^{2}\right)\end{array}$ & $\begin{array}{c}\Delta \mathbf{q}_{\text {total }}(0-5 \mathrm{kHz}) \\
\left(\mathrm{W} / \mathrm{m}^{2}\right)\end{array}$ \\
\hline \multicolumn{3}{|l|}{ Location PS2 } \\
\hline 1 & 3740 & 3636 \\
\hline 2 & 2183 & 1438 \\
\hline 3 & 53,365 & 16,186 \\
\hline 4 & 3158 & 3131 \\
\hline 5 & \multicolumn{2}{|c|}{1116} \\
\hline 6 & 3218 & 1638 \\
\hline 7 & 668 & 663 \\
\hline \multicolumn{3}{|l|}{ Location SS1 } \\
\hline 1 & 3861 & 3685 \\
\hline 2 & 2075 & 1321 \\
\hline 3 & 37,280 & 9769 \\
\hline 4 & 1873 & 1849 \\
\hline 5 & \multicolumn{2}{|c|}{668} \\
\hline 6 & 2201 & 990 \\
\hline 7 & 398 & 392 \\
\hline
\end{tabular}

The results demonstrate several differences from the results for location PS1 (Table 3.10). The results from Method 1 for both PS2 and SS1 do not agree well with the single-scale model results (although they are of the right order of magnitude), while for PS1 the value for Method 1 was in good agreement with the single-scale model. Method 4 demonstrates similar results as the results for PS1, where the multiple-scale model prediction was approximately 3 times higher than the single-scale model value. Methods 6 and 7 also give similar results for these two locations, where the results of data combined out to $5 \mathrm{kHz}$ between the two methods bound the single-scale model value.

The frequency range for Method 4 that matches the measured and single-scale model results was investigated for both of these locations, centering the range on the 
frequency corresponding to the measured integral length scale for each location, $700 \mathrm{~Hz}$ and $650 \mathrm{~Hz}$ for PS2 and SS1, respectively. The frequency range was approximately 30 $\mathrm{Hz}$ for both locations. The coherence for both of these locations (shown in Figure 3.27) was lower than for location PS1, and both PS2 and SS1 show more of a sharp peak near the frequency of the integral length scale. The coherence, combined with the smaller increase in heat flux results in a narrower frequency band to match the measured increase.

\subsubsection{Application of Multiple-scale Model to Other Data}

The multiple-scale model was applied to the cylinder stagnation region data of Diller and Van Fossen (1996), which allows the model to be tested on a different geometry and flowfield. The requirements for application of the multiple-scale model were measurements of steady heat transfer with and without freestream turbulence, as well as time-resolved velocity and heat flux data. The model was applied to the data for grid 42 in Diller and Van Fossen's data for the hot-wire location closest to the surface of the cylinder. The results for each of the methods presented earlier are listed in Table 3.12 below. Note that the data in the second column are included in a range up to $2 \mathrm{kHz}$ (as opposed to $5 \mathrm{kHz}$ in the earlier data) since the coherence range for this data was only 2 $\mathrm{kHz}$. It should be mentioned that none of the data analyzed from the work of Diller and Van Fossen demonstrated significant levels of coherence, with most values measured as less than 0.2 .

Table 3.13: Multiple-scale model results by various methods for data from Diller and Van Fossen (1996)

\begin{tabular}{|c|c|c|}
\hline Method & $\begin{array}{c}\Delta \mathbf{q}_{\text {total }} \\
\left(\mathbf{W} / \mathbf{m}^{\mathbf{2}}\right)\end{array}$ & $\begin{array}{c}\Delta \mathbf{q}_{\text {total }}(\mathbf{0}-\mathbf{2} \mathbf{k H z}) \\
\left(\mathbf{W} / \mathbf{m}^{\mathbf{2}}\right)\end{array}$ \\
\hline 1 & 974 & 927 \\
\hline 2 & 139 & 24 \\
\hline 3 & 33,770 & 14,421 \\
\hline 4 & 281 & 243 \\
\hline 5 & \multicolumn{2}{|c|}{1937} \\
\hline 6 & 5697 & 1118 \\
\hline 7 & 149 & 135 \\
\hline
\end{tabular}


For this data set, it appears that none of the various methods are good predictors of the measured heat transfer, except Method 6, which under predicts the increase, but is relatively close to the measured value compared to the other methods. The low coherence between the time-resolved velocity and heat flux data, when applied to the model data, greatly reduces the modeled values. 


\section{Chapter 4}

\section{CONCLUSIONS}

\subsection{SUMMARY}

The primary goals of this work were to measure and analytically model the increases in heat transfer due to the generated high intensity, large scale freestream turbulence field based on measured time-averaged baseline (low turbulence) and high turbulence surface heat transfer and on time-resolved measurements of surface heat flux and local fluctuating velocity. Along the way, it was hoped that the model and its application would shed some light onto the mechanism by which freestream velocity fluctuations affect surface heat transfer.

Section 3.2.3 presented the low turbulence heat transfer coefficients along with the measured high turbulence heat transfer coefficients. The increase in heat transfer was shown to be approximately two times higher on the pressure surface than on the suction surface (17\% on the pressure surface versus $8 \%$ on the suction surface). This result is consistent with observations of other researchers. This corresponds to increases in blade surface temperature of $5-10 \%$, which can significantly reduce the life of a turbine blade

The analytical model was presented in two forms, documented as the "singlescale" and "multiple-scale" models. The results presented in sections 3.4.2 and 3.4.3 demonstrated how the single-scale model developed predicted the measured increases in heat transfer for the data in this work, as well as for the data from the work of various other researchers in varying flowfields and on different geometries (turbine vane, turbine blade, cylinder stagnation region and flat plate). The model was shown to fairly accurately predict the increases in heat transfer, except in regions of turbulent flow and transition. The model was shown to slightly over predict the heat transfer for turbulent boundary layers and demonstrated a deficiency in predicting early laminar to turbulent boundary layer transition caused by elevated levels of freestream turbulence. 
The multiple-scale model development provided some interesting insight into the frequencies of turbulent eddies which are most effective at increasing surface heat transfer. It was shown that the time-resolved velocity data could be transformed into the frequency domain and decomposed into the individual eddy sizes (scales or frequencies), and that the energy associated with each individual scale of eddy could be determined. The multiple-scale model then applied the analytical model developed to each eddy scale to determine a spectrum of heat flux increase. The time-resolved heat flux data was used to compare with this spectrum of increases and also to determine the coherence between the fluctuating velocity and surface heat transfer. The coherence and the measured heat flux spectra demonstrated that there is a small band of frequencies for which freestream velocity fluctuations affect surface heat transfer, roughly centered around the frequency of the integral length scale (most energetic eddies) of the freestream turbulence. This result was in good agreement with previous work by Dullenkopf and Mayle (1995) and Mayle, et al. (1998).

The multiple-scale model attempted to determine how to combine the spectrum of increases in heat transfer by various methods with moderate success. The resultant predicted increases were on the right order of magnitude with the measured increases, but were approximately a factor of 3 greater.

\section{What was learned:}

1. The single-scale model, developed based on a physical model, demonstrated good ability, within certain limitations, at predicting increases in heat transfer due to freestream turbulence. Based on the results, the mechanism of the model seems reasonable.

2. The current data set agrees well with existing correlations, although there is some discrepancy with the suction surface data.

3. Both measured heat flux spectra and coherence between freestream velocity and surface heat transfer fluctuations are valuable tools in analyzing the frequencies (eddy sizes) of turbulence that are effective at increasing surface heat transfer. 
4. The analytical model assumption of large-scale eddies penetrating the boundary layer and superimposing their energy in the form of heat flux increases on the mean heat flux appears to be reasonable. The frequencies in the measured spectra and coherence functions where energy and coherence are attenuated correlate well with the integral length scale and effective turbulence frequencies presented by Mayle, et al. (1998). Above these frequencies, the effectiveness of the turbulent freestream eddies at increasing heat transfer decreases rapidly due to viscous dissipation of energy at higher frequencies (smaller scales).

5. The heat flux increases in the multiple-scale model do not seem to add as the RMS of the increases of the individual eddies. The single-scale model, where the increase is a function of the combined turbulent energy at the frequency of the integral scale, was much more effective at predicting increases in surface heat transfer due to turbulence.

\subsection{ApPlication to Turbine Design}

The results of the time-resolved measurements provide a very limited data set that is applicable to design of transonic rotor blades. The measurements were limited to three blade locations that have been reported in previous work in the transonic facility. The results of these measurements agree well with existing correlations. The application of the analytical model developed has reasonable value to turbine designers, within limitations. The model requires knowledge of the local turbulence intensity (RMS of freestream velocity fluctuations near the edge of the boundary layer) and integral length scale. These values may not be known for real engine conditions, although computational fluid dynamics predictions may be able to determine a reasonable distribution of these values as a function of the inlet turbulence conditions, making the model moderately applicable to engine development. The results of the multiple-scale model analysis do, however, provide insight into the scales of turbulence that are most effective at affecting surface heat transfer. 


\subsection{OUTLOOK AND FUTURE WORK}

The present investigation generated turbulence that is consistent with recent reports of the turbulent flowfield from modern gas turbine combustors entering the first stage rotor blades. The turbulence intensity and scale were not varied to provide a more extensive data set for the analyses. Previous work in this facility varied intensity and scale; however, neither was consistent with what is currently believed to consistent with real engine conditions. Future work should vary the intensity and scale (within reasonable limits compared to the model of engine conditions) and should investigate more gage locations. Gages currently being developed provide a higher density of measurement locations with a smaller spatial resolution. Provided that the time-response of these gages is sufficient to resolve the scales of turbulence present in the flowfield, more gage locations can be sampled simultaneously. This provides more measurement locations and higher spatial resolution, but will also allow for cross correlation of the time signals from various gages. Cross correlation in the time domain adds the ability to track the progression of eddies in the boundary layer, providing an additional analysis technique.

Within the measurement limitations of the facility, the state of the boundary layer and the details of the boundary layer thickness and growth should be determined. These details were not resolved in this work, but with great care optical techniques such as shadowgraph and Schleiren photography and oil flow visualization should provide decent approximations of these parameters.

Finally, similar analyses should be performed with a turbulent boundary or film layer. Film cooling and turbulent boundary layers have associated energy and scales of turbulence that can be measured with techniques similar to those presented in this work. The analytical model application could be tested under these conditions to test its validity. 


\section{REFERENCES}

Ames, F.E. and Moffat, R.J., 1990, "Heat Transfer with High Intensity, Large Scale Turbulence: The Flat Plate Turbulent Boundary Layer and Cylindrical Stagnation Point," Stanford University Report No. HMT-44.

Ames, F.E., 1994, "Experimental Study of Vane Heat Transfer and Aerodynamics at Elevated Levels of Turbulence," NASA Contractor Report 4633.

Ames, F.E., 1995, "The Influence of Large-Scale High-Intensity Turbulence on Vane Heat Transfer," ASME Journal of Turbomachinery, Vol. 119, pp. 23-30.

Ames, F.E., 1997, “Aspects of Vane Film Cooling with High Turbulence - Part I: Heat Transfer," ASME 97-GT-239.

Ames, F.E., 1997, “Aspects of Vane Film Cooling with High Turbulence - Part II: Adiabatic Effectiveness,” ASME 97-GT-240.

Ashworth, D.A., LaGraff, J.E. and Schultz, D.L., 1989, "Unsteady Interaction Effects on a Transitional Turbine Blade Boundary Layer," ASME Journal of Turbomachinery, Vol. 111, pp. 162-168.

Baker, K.I. and Diller, T.E., 1993, "Unsteady Heat Flux and Temperature Measurements," ASME/AIChE National Heat Transfer Conference.

Barrett, M.J. and Hollingsworth, D.K., 2003, "Correlating Friction Velocity in Turbulent Boundary Layers Subjected to Free-stream Turbulence," AIAA paper No. J26247.

Barringer, M.D., Richard, O.T., Walter, J.P., Stitzel, S.M., and Thole, K.A., 2002, "Flowfield Simulations of a Gas Turbine Combustor," ASME Journal of Turbomachinery, Vol. 124, pp. 508-516.

Blair, M.F., 1983, "Influence of Free-Stream Turbulence on Turbulence Boundary Layer Heat Transfer and Mean Profile Development, Parts I and II," ASME Journal of Heat Transfer, pp. 33-47.

Blevins, R.D., 1994, Flow-Induced Vibration, $2^{\text {nd }}$ Ed., Krieger Publishing. 
Boyle, R. J., Lucci, B.L., Verhoff, V.G., Camperchioli, W.P. and La, H., 1998, "Aerodynamics of a Transitioning Turbine Stator Over a Range of Reynolds Numbers," ASME Paper 98-GT-295 (NASA/TM-1998-208408).

Bradshaw, P., 1974, "Effect of Free-Stream Turbulence on Turbulent Sheer Layers," ARC Paper 35648.

Bubb, J.V., 1999, "The Influence of Pressure Ratio on Film Cooling Performance of a Turbine Blade," MS Thesis, Virginia Tech.

Castro, I.P., 1984, "Effects of Freestream Turbulence on Low Reynolds Number Boundary Layer Flows," ASME Journal of Fluids Engineering, Vol. 106, pp. 298-306.

Denninger, M.J. and Anderson, A.M., 1999, “An Experimental Study on the Relationship Between Velocity Fluctuations and Heat Transfer in a Turbulent Air Flow," ASME Journal of Turbomachinery, Vol. 121, pp. 288-294.

Diller, T.E. and Van Fossen, G.J., 1996, Unpublished unsteady heat transfer and hot wire data to a cylinder model, Work performed at NASA Glenn.

Dullenkopf, K. and Mayle, R.E., 1995, "An Account of Free-Stream-Turbulence Length Scale on Laminar Heat Transfer," Darryl E. Metzger Memorial Session Paper, ASME Journal of Turbomachinery, Vol. 117, pp. 401-406.

Dunn, M.G., Martin, H.L. and Stanek, M.J., 1986, "Heat Flux and Pressure Measurements and Comparison with Prediction for a Low-Aspect-Ratio Turbine Stage," ASME Journal of Turbomachinery, Vol. 108, pp. 108-115.

Dunn, M.G., Seymour, P.J., Woodward, S.H., George, W.K. and Chupp, R.E., 1989, "Phase-Resolved Heat-Flux Measurements on the Blade of a Full-Scale Rotating Turbine," ASME Journal of Turbomachinery, Vol. 111, pp. 8-19.

Garside, T., Moss, R.W., Ainsworth, R.W., Dancer, S.N., Rose, M.G., 1994, "Heat Transfer to Rotating Turbine Blades in a Flow Undisturbed by Wakes," IGTI Aeroengine Congress and Expo, The Hague, Netherlands, ASME 94-GT-94.

Giel, P.W., Bunker, R.S., Van Fossen, G.J. and Boyle, R.J., 2000, "Heat Transfer Measurements and Predictions on a Power Generation Gas Turbine Blade," ASME 2000GT-0209.

Goldstein, R.J., Lau, K.Y. and Leung, C.C., 1983, "Velocity and Turbulence Measurements in Combustion Systems," Experiments in Fluids, 1, pp. 93-99.

Guenette, G.R., Epstein, A.H., Giles, M.B., Haimes, R. and Norton, R.J.G., 1989, "Fully Scaled Transonic Turbine Rotor Heat Transfer Measurements," ASME Journal of Turbomachinery, Vol. 111, pp. 1-7. 
Hager, J. M., Simmons, S., Smith, D., Onishi, S., Langley, L. W., and Diller, T. E., "Experimental Performance of a Heat Flux Microsensor." ASME Journal of Engineering for Gas Turbines and Power, Vol. 113, 1991b, pp. 246-250.

Hancock, P.E. and Bradshaw, P., 1983, "The Effect of Free-Stream Turbulence on Turbulent Boundary Layers," ASME Journal of Fluids Engineering, Vol. 105, p.284-289.

Hinze, J., 1975, Turbulence, $2^{\text {nd }}$ Ed., McGraw-Hill, New York.

Holmberg, D.G., and Diller, T. E., "High Frequency Heat Flux Sensor Calibration and Modeling." ASME Journal of Fluids Engineering, Vol. 117, December 1995, pp. 659-664.

Holmberg, D.G. and Pestian D.J., 1996, "Wall-Jet Turbulent Boundary Layer Heat Flux, Velocity, and Temperature Spectra and Time Scales.” ASME 96-GT-529.

Holmberg, D.G., 1996, “A Frequency Domain Analysis of Surface Heat Transfer/Freestream Turbulence Interactions in a Transonic Turbine Cascade," Ph.D. Dissertation, Virginia Tech.

Incropera, F. P. and DeWitt, D. P., Fundamentals of Heat and Mass Transfer, John Wiley and Sons, New York, 1990, p. 259-262.

Johnson, L.P., 1995, "Effects of Freestream Turbulence on Turbine Blade Heat Transfer in Transonic Flow," MS Thesis, Virginia Tech.

Johnston, C.A., Bogard, D.G. and McWaters, M.A., 1999, "Highly Turbulent Mainstream Effects on Film Cooling of a Simulated Airfoil Leading Edge." ASME 99GT-261.

Kays, W.M. and Crawford, M.E., 1993, Convective Heat and Mass Transfer, $3^{\text {rd }}$ Ed., McGraw-Hill, New York.

Kestin, J. and Wood, R.T., 1971, "The Influence of Turbulence on Mass Transfer to Cylinders," ASME Journal of Heat Transfer, Vol. 93, pp. 321-327.

Kuotmos, P. and McGuirk, J.J., 1989, "Isothermal flow in a gas turbine combustor - a benchmark experimental study," Experiments in Fluids, 7, pp. 344-354.

Lowery, G.W. and Vachon, R.I., 1975, "The Effect of Turbulence on Heat Transfer from Heated Cylinders," Int. Journal of heat and Mass Transfer, Vol. 18, pp. 1229-1242.

Maciejewski, P.K. and Anderson, A.M., 1996, "Elements of a General Correlation for Turbulent Heat Transfer," ASME Journal of Heat Transfer, Vol. 118, pp. 287-293. 
Maciejewski, P.K. and Moffat, R.J., 1989, "Heat Transfer with Very High Free Stream Turbulence,” Stanford University Report No. HMT-42.

Maciejewski, P.K. and Moffat, R.J., 1992, "Heat Transfer with Very High Free Stream Turbulence: Part I - Experimental Data," ASME Journal of Heat Transfer, Vol. 114 , pp. 827-833.

Maciejewski, P.K. and Moffat, R.J., 1992, "Heat Transfer with Very High Free Stream Turbulence: Part II - Analysis of Results," ASME Journal of Heat Transfer, Vol. 114, pp. 834-839.

MacMullin, R., Elrod, W. and River, R., 1989, "Free-Stream Turbulence From a Circular Wall Jet on a Flat Plate Heat transfer and Boundary Layer Flow," ASME Journal of Turbomachinery, Vol. 111, pp. 78-86.

Mayle, R.E., Dullenkopf, K. and Schulz, A., 1998, "The Turbulence That Matters," 1997 Best Paper Award, Heat Transfer Committee, ASME Journal of Turbomachinery, Vol. 120, pp. 402-409.

Mehendale, A.B., Han, J.C. and Ou, S., 1991, "Influence of Mainstream Turbulence on Leading Edge Heat Transfer," ASME Journal of Heat Transfer, Vol. 113, pp. 843-850.

Mehendale, A.B. and Han, J.C., 1992, "Influence of High Mainstream Turbulence on Leading Edge Film Cooling Heat Transfer," ASME Journal of Turbomachinery, Vol. 114, pp. 707-715.

Mehendale, A.B., Ekkad, S.V. and Han, J.C., 1994, "Mainstream Turbulence Effect on Film Effectiveness and Heat Transfer Coefficient of a Gas Turbine Blade with Air and $\mathrm{CO}_{2}$ Film Injection," Intl. Journal of Heat and Mass Transfer, Vol. 37, pp. 2707-2714.

Moffat, R.J., 1988, “Describing Uncertainties in Experimental Results,” Exp. Thermal and Fluid Science, Elsevier Science Publishing Co, Inc, New York, 1988, pp. 317.

Moss, R.W. and Oldfield, M.L.G., 1991, "Measurements of Hot Combustor Turbulence Spectra," ASME 91-GT-351.

Moss, R.W., and Oldfield, M.L.G., 1992, "Measurements of the Effect of FreeStream Turbulence Length Scale on Heat Transfer," ASME 92-GT-244.

Moss, R.W., and Oldfield, M.L.G., 1996, "Effect of Free-Stream Turbulence on FlatPlate Heat Flux Signals: Spectra \& Eddy Transport Velocities," ASME J.ournal of Turbomachinery, Vol. 118, pp.461-467.

Nix, A.C., 1996, "Effects of Shock Wave Passing on Turbine Blade Heat Transfer in a Transonic Cascade," MS Thesis, Virginia Tech. 
Nix, A.C., Diller, T.E. and Ng, W.F., 1997a, "Experimental Evaluation of Heat Transfer Effects of Shock Waves on Transonic Turbine Blades," ASME 97-WA/HT-1.

Nix, A.C., Reid, T., Peabody, H., Ng, W.F., Diller, T.E. and Schetz, J.A., 1997b, "Effects of Shock Wave Passing on Turbine Blade Heat Transfer in a Transonic Cascade," AIAA-97-0160.

Nix , A.C., Smith, A.C., Diller, T.E., Ng, W.F. and Thole, K.A., 2001, "High Intensity, Large Length-Scale Freestream Turbulence Generation in a Transonic Cascade," ASME GT-2002-30523.

Polanka, M.D., 1999, "Detailed Film Cooling Effectiveness and Three Component Velocity Field Measurements on a First Stage Turbine Vane Subject to High Freestream Turbulence," Ph.D. Dissertation, The University of Texas at Austin.

Popp, O., Smith, D.E., Bubb, J.V., Grabowski III, H.C., Diller, T.E., Schetz, J.A. and Ng, W.F., 1999, "Steady and Unsteady Heat Transfer in a Transonic Film Cooled Turbine Cascade," ASME 99-GT-259.

Popp, O., 1999, "Steady and Unsteady Heat Transfer in a Film Cooled Transonic Turbine Cascade," Ph.D Dissertation, Virginia Tech.

Popp, O., Smith, D.E., Bubb, J.V., Grabowski, H.C., Diller, T.E., Schetz, J.A. and $\mathrm{Ng}$, T.E., 2000, "Investigation of Heat Transfer in a Film Cooled Transonic Turbine Cascade, Part II: Unsteady Heat Transfer,” ASME 2000-GT-203.

Radomsky, R.W. and Thole K.A., 1998, "Effects of High Freestream Turbulence Levels and Length Scales on Stator Vane Heat Transfer," ASME 98-GT-236.

Radomsky, R.W. and Thole K.A., 2000, "Flowfield Measurements for a Highly Turbulent Flow in a Stator Vane Passage," ASME Journal of Turbomachinery, Vol. 122, pp. 255-262.

Radomsky, R.W. and Thole K.A., 2002, "Detailed Boundary Layer Measurements on a Turbine stator Vane at Elevated Freestream Turbulence Levels," ASME Journal of Turbomachinery, Vol. 124, pp. 107-118.

Sahm, M.K. and Moffat, R.J., 1992, "Turbulent Boundary Layers with High Turbulence: Experimental Heat Transfer and Structure on Flat and Convex Walls," Stanford University Report No. HMT-45.

Sauer, J., 1996, “The SUDI Turbulence Generator - A Method to Generate High Freestream Turbulence Levels and a Range of Length Scales," Thesis, University of Wisonsin / University of Karlsruhe, Germany. 
Schauer, J.J and Pestian, D.J., 1996, "Film Cooling Heat Transfer with High Freestream Turbulence," ASME 96-WA/HT-6.

Smith, D.E., Bubb, J.V., Popp, O., Diller, T.E. and Hevey, S.J., 1999, “A Comparison of Radiation Versus Convection Calibration of Thin-Film Heat Flux Gauges," Proceedings of the ASME Heat Transfer Division - 1999, HTD-Vol. 364-4, pp. 79-84.

Smith, D. E., 1999, “An Investigation of Heat Transfer Coefficient and Film Cooling Effectiveness in a Transonic Turbine Cascade," MS Thesis, Virginia Tech.

Smith, D.E., Bubb, J.V., Popp, O., Grabowski, H.C., Diller, T.E., Schetz, J.A. and Ng, T.E., 2000, "Investigation of Heat Transfer in a Film Cooled Transonic Turbine Cascade, Part I: Steady Heat Transfer,” ASME 2000-GT-202.

Smith, M.C. and Keuthe, A.M., 1966, "Effects of Turbulence on Laminar Skin Friction and Heat Transfer," Physics of Fluids, Vol. 9, pp. 2337-2344.

Thole, K.A., Bogard, D.G. and Whan-Tong, J.L., 1994, “Generating High Freestream Turbulence Levels," Experiments in Fluids 17, 375-380.

Thole, K.A. and Bogard, D.G., 1995, "Enhanced Heat Transfer and Shear Stress Due to High Free-Stream Turbulence," ASME Journal of Turbomachinery, Vol. 117, pp. 418424.

Van Fossen, G.J. and Simoneau, R.J., 1985, "Preliminary Results of a Study of the Relationship Between Free Stream Turbulence and Stagnation Region Heat Transfer," ASME 85-GT-84.

Van Fossen, G.J., Simoneau, R.J. and Ching, C.Y., 1995, "Influence of Turbulence Parameters, Reynolds Number, and Body Shape on Stagnation Region Heat Transfer," ASME Journal of Heat Transfer, Vol. 117, pp.597-603.

Van Fossen, G.J. and Bunker, R.S., 2002, “Augmentation of Stagnation Region Heat Transfer Due to Turbulence from an Advanced Dual-Annular Combustor," ASME GT2002-30184.

Wang, H.P., Goldstein, J., and Olson, R.J., 1999, "Effect of High Freestream Turbulence with Large Scale on Blade Heat/Mass Transfer," ASME Journal of Turbomachinery, Vol. 121, pp. 217-224.

Wesner, A., 1996, "A Single-plate Interferometric Study of the Unsteady Density Field in a Transonic Cascade," Ph.D Dissertation, Virginia Tech.

Zerkle, R.D. and Lounsbury, R.J., 1988, "Freestream Turbulence Effect on Turbine Airfoil Heat Transfer," J. Propulsion, Vol. 5, pp. 82-88. 


\section{APPENDIX A}

\section{Turbulence Generation Paper and Presentation}

The following pages detail the development and testing of the turbulence generation grid in the form of a paper and presentation. The paper, written by the author of this dissertation and submitted to the 2002 IGTI conference in Amsterdam, NL, details the design and testing of the turbulence generation grid. The presentation given by the author at the conference follows the paper and includes additional data (hot-wire surveys) not included in the paper. 


\title{
GT-2002-30523
}

\section{HIGH INTENSITY, LARGE LENGTH-SCALE FREESTREAM TURBULENCE GENERATION IN A TRANSONIC TURBINE CASCADE}

\author{
A.C. Nix, A.C. Smith, T.E. Diller, W.F. Ng, K.A. Thole \\ Virginia Polytechnic Institute and State University \\ Blacksburg, VA 24061
}

\section{ABSTRACT}

Heat transfer predictions in gas turbine engines have focused on cooling techniques and on the effects of various flow phenomena. The effects of wakes, passing shock waves and freestream turbulence have all been of primary interest to researchers. The focus of the work presented in this paper is to develop a turbulence grid capable of generating high intensity, large-scale turbulence for use in experimental heat transfer measurements in a transonic facility. The grid is desired to produce freestream turbulence characteristic of the flow exiting the combustor of advanced gas turbine engines. A number of techniques are discussed in this paper to generate high intensity, large length-scale turbulence for a transonic facility. Ultimately, the passive grid design chosen is capable of producing freestream turbulence with intensity of approximately $10-12 \%$ near the entrance of the cascade passages with an integral length-scale of $2 \mathrm{~cm}$.

\section{NOMENCLATURE}

$\begin{array}{ll}\mathrm{c} & \text { Blade chord length } \\ \mathrm{d}_{1} & \text { Original grid bar diameter }-2.54 \mathrm{~cm}(1 \mathrm{in} .) \\ \mathrm{d}_{2} & \text { Modified grid bar diameter- } 5.08 \mathrm{~cm}(2 \mathrm{in} .) \\ \mathrm{D}_{\mathrm{w}} & \text { Hot-wire probe diameter }(5 \mu \mathrm{m}) \\ \mathrm{E}(\mathrm{f}) & \text { Energy spectra of streamwise velocity component } \\ \mathrm{f} & \text { Frequency } \\ \mathrm{I} & \text { Momentum flux ratio }\left(\rho_{\mathrm{j}} \mathrm{u}_{\mathrm{j}}{ }^{2} / \rho_{\infty} \mathrm{d}_{\infty}{ }^{2}\right) \\ \mathrm{k}_{\mathrm{m}} & \text { Thermal conductivity of air at mean temperature } \\ \mathrm{M} & \text { Mass flux ratio }\left(\rho_{\mathrm{j}} \mathrm{u}_{\mathrm{j}} / \rho_{\infty} \mathrm{d}_{\infty}\right) \\ \mathrm{Ma} & \text { Mach number } \\ \mathrm{Nu} & \text { Nusselt number of hot-wire } \\ \mathrm{P}_{\mathrm{s}} & \text { Freestream static pressure } \\ \mathrm{P}_{\mathrm{t}} & \text { Freestream total pressure } \\ \mathrm{R}_{11} & \text { Autocorrelation of streamwise velocity component } \\ \mathrm{Re}^{\prime} & \text { Reynolds number } \\ \mathrm{Re}_{\mathrm{w}} & \text { Reynolds number of hot-wire } \\ T & \text { Integral time scale } \\ \mathrm{T}_{\mathrm{m}} & \text { Mean temperature }\left(\left(\mathrm{T}_{\mathrm{w}}+\mathrm{T}_{\mathrm{s}}\right) / 2\right)\end{array}$

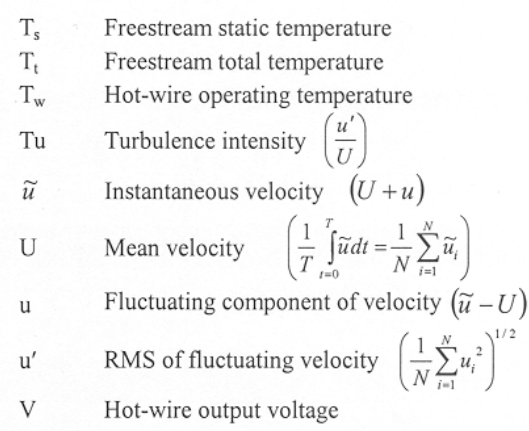

\section{Greek Symbols}

$\begin{array}{ll}\kappa & \text { Wave number }(2 \pi \mathrm{f} / \mathrm{U}) \\ \Lambda_{\mathrm{x}} & \text { Integral length-scale } \\ \rho & \text { Density of air } \\ \rho_{\mathrm{m}} & \text { Density of air at mean temperature } \\ \tau & \text { Autocorrelation time } \\ \mu_{\mathrm{m}} & \text { Dynamic viscosity of air at mean temperature }\end{array}$

\section{INTRODUCTION}

The desire for increased engine efficiency in modern gas turbine engines has led to increases in thermal load on high-pressure turbine blading due to high combustor exit temperatures. The flowfield in the turbine section is highly turbulent as a result of combustion dynamics and blade row interactions. Prediction of blade surface temperatures therefore requires researchers to study the effects of unsteady flow phenomena on turbine blade surface heat transfer. These unsteady phenomena include wakes and shock waves due to rotor-stator blade passing and freestream turbulence mainly generated in the combustor. The present research focuses on the effects of combustor exit 
freestream turbulence on blade surface heat transfer and film cooling effectiveness.

In order to study the realistic effect turbulence has on heat transfer in an experimentally controlled environment, the turbulence must be generated to model combustor exit turbulence. Several research groups have generated high levels of freestream turbulence using different techniques that were investigated specifically for their applicability in a transonic blow down cascade wind tunnel facility. These techniques can be categorized into mock combustor/jets in crossflow and passive and active grids. The majority of the previous research with high levels of freestream turbulence has been in wind tunnel facilities with relatively low speed flows. These facilities typically concentrate on matching engine representative Reynolds number $(\mathrm{Re})$. The facility for this work is a transonic facility that matches closely with engine Reynolds number, as well as Mach number (Ma). The high velocity, slightly compressible flow at the inlet of the cascade poses a problem in generation of high levels of freestream turbulence.

This paper focuses on the development of a turbulence grid designed to produce high intensity, large length-scale freestream turbulence in high-speed flow. The generator was designed to produce freestream turbulence with a turbulence intensity $(\mathrm{Tu})$ of approximately $15-20 \%$ with an integral length scale of $3-4 \mathrm{~cm}$. This desired turbulence level (intensity and scale) was determined by matching results by Van Fossen [1]. He used a $60^{\circ}$ section of a GE 90 combustor in a wind tunnel to model the exit flow of a combustor, including inlet swirl vanes, film cooling holes and dilution holes. All three of these combustor components contribute strongly to the combustor exit turbulence conditions. Hot-wire surveys were performed downstream of the combustor section to determine turbulence intensity and length scale. Combustion was not included, but previous research by Moss and Oldfield [2] showed that turbulence intensity and length scale were not significantly affected by the presence of combustion. Van Fossen measured turbulence intensities as high as $20 \%$ with length-scales between 1 and $1.5 \mathrm{~cm}$. Using these results, the desired length scale was based on matching the ratio of length scale to blade chord length $\left(\Lambda_{x} / \mathrm{c}\right)$ of the transonic turbine cascade facility to the blades of a GE 90 engine.

In performing this research, a methodical approach was taken. First, grid turbulence was generated to compare with previously reported data under low speed conditions. Second, the grid was tested in the transonic wind tunnel to quantify the turbulence and compare the results with the low speed testing. Third, based on inadequate turbulence results of the initial design, the grid was modified to the current design. Results of measured mean and turbulent quantities will be presented.

\section{REVIEW OF GENERATING TECHNIQUES Mock Combustor/Jets in Crossflow}

Ames [3] designed a mock combustor where air was directed through a liner into the mainstream flow. The main flow is directed along the walls of the liner and two rows of holes were used to simulate dilution injection flows. Turbulence levels of $13 \%$ were achieved with an integral length scale of $1.6 \mathrm{~cm}$. The ratio of length scale to true chord length was $\Lambda_{\mathrm{x}} / \mathrm{c}=0.11$. A similar combustor simulator, designed by Wang, Goldstein and Olson [4], had a front panel on the inlet to the simulator with four slots and guide vanes on the inside to force a swirling flow along the sidewalls of the simulator. Rows of holes were placed along the sidewalls to simulate cooling flow and dispersion jets. The highest turbulence parameters achieved were a turbulence intensity of $18 \%$ and length scale of $8 \mathrm{~cm}$ $\left(\Lambda_{\mathrm{x}} / \mathrm{c}=0.43\right)$.

Schauer and Pestian [5] designed a turbulence generator where secondary flow was injected into the mainstream from two opposing rows of holes located upstream of the test section. The jet to freestream velocity ratio was 14 . Turbulence parameters achieved were $17 \%$ turbulence intensity and length scale was $8 \mathrm{~cm}[6]$.

Thole, et al. [7] used small, high velocity normal jets injected into cross flow. A splitter plate was placed between the jets to prevent interaction of the jets. Further improvements using a downstream cylinder were made by Harrington et al. [8] to reduce bulges in the mean flow. The turbulence parameters achieved were up to $20 \%$ intensity and $2-3 \mathrm{~cm}$ length scale. Later work by Thole led to the design of a large-scale combustor simulator in a low speed, close-loop wind tunnel facility. The combustor simulator, detailed in Barringer et al. [9] produced turbulence intensities of $18 \%$ and length scale of 6.5 $\mathrm{cm}\left(\Lambda_{\mathrm{x}} / \mathrm{c}=0.11\right)$, using large dilution jets.

\section{Grid or Bar Generators}

Mehendale and Han [10] used an active grid made of hollow brass tubes with holes drilled at the grid intersections. Blowing was done uniformly in the co-flow (downstream) direction only, with injection ratio (injection mass flow rate/freestream mass flow rate) of $2.5 \%$. Turbulence intensity of $12.9 \%$ was reported, but flow uniformity was reportedly not good.

Sauer and Thole [11] and Radomsky and Thole [12] developed a turbulence generator with simultaneous upstream and downstream blowing from vertical bars. The generator was designed to produce turbulence up to $20 \%$ intensity and a range of length-scales by adjusting bar spacing and blowing rates.

Polanka and Bogard [13] developed a turbulence generator which creates turbulence up to $20 \%$ in a passive bar arrangement. The generator provided high intensity, large-scale turbulence with good flow uniformity by designing a bar grid with large flow blockage.

Boyle et al. [14] produced turbulence using parallel round bars with relatively large flow blockage and the option of active blowing. Turbulence of greater than $10 \%$ was produced with approximately 1.5 $\mathrm{cm}$ length scale. It was shown that active blowing significantly improved flow uniformity but reduced overall turbulence. Giel et al [15] produced turbulence in a transonic facility using an active blowing grid of square bars. The grid incorporated blowing in the upstream direction only at a rate of approximately $5 \%$ of the cascade mass flow rate. Blowing air supply pressure to the grid was 125 psig $(965 \mathrm{kPa})$. Turbulence intensity and length scale were $10 \%$ and 2.2 $\mathrm{cm}\left(\Lambda_{\mathrm{x}} / \mathrm{c}=0.17\right)$, respectively. The work by Giel was of significant interest, since the facility is a transonic tunnel with inlet Mach number similar to the blowdown facility for this study. This was the only other study producing high intensity, large-scale turbulence in a transonic facility.

\section{EXPERIMENTAL APPARATUS AND INSTRUMENTATION Low Speed Facility}

The setup for low speed testing of the grid consisted of a $5 \mathrm{HP}$ blower attached to several transition pieces, followed by a test section. The blower created a mainstream flow velocity of approximately 20 $\mathrm{m} / \mathrm{s}$ at the entrance to the test section, whereas the inlet velocity of the transonic wind tunnel is approximately $120 \mathrm{~m} / \mathrm{s}$. The low speed test setup was designed such that the inlet geometry was identical to the transonic facility from the grid downstream to the test section. The cross-sectional area at the grid location is $38.1 \mathrm{~cm}$ (15 in.) by $22.86 \mathrm{~cm}$ ( 9 in.) and is followed by a 2-D contraction to the test section area. A 
Plexiglas test section $51 \mathrm{~cm}$ (20 in.) in length, $15.2 \mathrm{~cm}$ (6 in.) wide and $30.5 \mathrm{~cm}$ (12 in.) high was fabricated to take data downstream of the turbulence grid. The cross sectional area of the test section was also designed to match the area of the transonic facility. A flow straightener section was installed in the setup to lower background turbulence levels to less than $2 \%$ upstream of the turbulence grid. Blowing air for the turbulence-generating grid was supplied through high-pressure lines to a plenum tank. The plenum supplied air to each of the three blowing bars of the grid.

\section{Transonic Wind Tunnel Facility and Cascade Test Section}

The experimental facility in which the turbulence grid was ultimately to be designed for use was a transonic blowdown cascade wind tunnel. Previous research in this facility has been published by Holmberg [16], Nix et al. [17,18], Smith et al. [19] and Popp et al. [20]. The facility allows for run times of up to 35 seconds with the inlet total pressure maintained by a feedback control scheme. The facility is capable of heated runs by way of a heating loop built into the tunnel upstream of the cascade test section. A schematic of the wind tunnel and cascade test section, including the turbulence grid, is shown in Figure 1.

The test section contains a cascade of four full and two half first stage rotor blades forming five passages. The turbine blades are scaled up three times to allow for a film cooling scheme and instrumentation on both surfaces of the blades. The span is $15.3 \mathrm{~cm}$ ( 6 in.) and the aerodynamic chord is $13.7 \mathrm{~cm}$ (5.4 in.). The Reynolds number based on aerodynamic chord and exit conditions is about $6 \cdot 10^{6}$. The inlet Mach number to the cascade test section is approximately 0.4 , with unheated flow mainstream velocity of approximately $120 \mathrm{~m} / \mathrm{s}$. Fo ease of installation and removal, the turbulence grid was designed to be placed directly upstream of the 2-D contraction entering the cascade normal to the inlet flow direction. Again, the geometry of the cross-sectional area at the grid location downstream to the test section was identical to the low-speed facility.

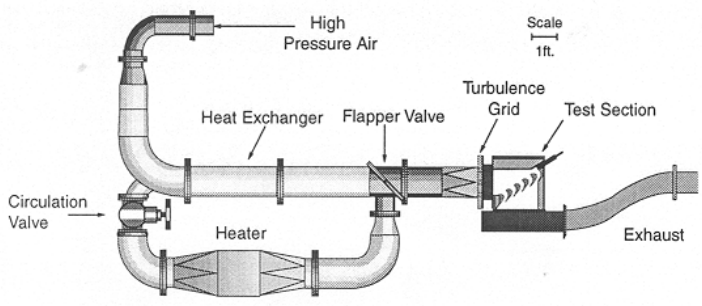

Figure 1. Transonic blowdown facility used for the experiments

\section{Instrumentation}

Pressure, temperature and velocity were measured in this study. In the low speed facility, blowing bar plenum pressure an temperature were measured. The bar plenum pressure taps were connected to $0-100 \mathrm{psi}$ pressure transducers. Freestream velocity upstream of the turbulence grid was measured using a pitot-static probe connected to a differential pressure transducer with a range of 2 inches of water. In the transonic facility, freestream total and static pressures were measured using a pitot-static probe, total pressure probe traverse and wall static pressure taps. All pressure taps were connected to $0-15$ psi differential pressure transducers. Temperature in the transonic facility was measured using a type $\mathrm{K}$ total temperature probe located upstream of the cascade test section. Time-resolved velocity was measured in both the low and high-speed facilities using a hot-wire anemometer. The hot-wire was $5 \mu \mathrm{m}$ in diameter and approximately $1.5 \mathrm{~mm}$ in length. Measurements were performed at an overheat ratio of 1.5 . The orientation of the wire was such that the wire was parallel to the span of the turbine blade. Two separate methods, one for each facility, were required to calibrate the hot-wire.

The hot-wire anemometer for low speed testing was calibrated using an air jet calibrator. The hot-wire probe was placed in a low turbulence air jet of known velocity and similar operating temperature as the low speed tunnel. The velocity of the jet was varied and the output voltage of the wire was measured and recorded. The hot-wire voltage vs. velocity data was then fit with a fourth order polynomial. Using this calibration curve, the time-resolved hot-wire voltage measurements performed in the low speed tunnel could be converted to velocity for calculation of turbulent quantities.

Use of the hot-wire probe to measure time resolved velocity in the transonic facility required a separate calibration of the probe. Use of the probe in a compressible flow field does not allow for a simple calibration of voltage versus velocity. To account for compressible flow, the wire was calibrated in-situ in the wind tunnel through a blowdown calibration. The wire was placed in the wind tunnel and the wire output voltage was recorded, along with the tunnel total temperature and total and static pressures from a pitot-static pressure probe located very close to the hot-wire probe. The tunnel was run and the total pressure was allowed to drop to very low values, unchoking the cascade and providing lower Reynolds numbers. The data from these measurements was then used to plot a calibration curve of wire Reynolds number $\left(\operatorname{Re}_{w}\right)$ and Nusselt number (Nu'). The data was then curve fit with a power law to give an equation of the type:

$$
N u^{\prime}=C \cdot \operatorname{Re}_{w}^{x}
$$

where the wire Reynolds number and Nusselt number are determined by:

$$
\begin{aligned}
& \operatorname{Re}_{w}=\frac{\rho_{m} \tilde{u} D_{w}}{\mu} \\
& N u^{\prime} \propto \frac{V^{2}}{k_{m}\left(T_{m}-T_{s}\right)}
\end{aligned}
$$

All wire properties were constant given a constant temperature anemometer that maintains wire resistances during the run.

Time-resolved data from the hot-wire during test runs could then be converted from hot-wire voltage to Nusselt number and the wire Reynolds number could be determined with the blowdown calibration curve. All values in Equations (2) and (3) could be measured during a test run, but the product $\left(\rho_{\mathrm{m}} \tilde{u}\right)$ could not be decoupled since the wire will respond to fluctuations in velocity as well as density. The product of density and velocity $\left(\rho_{\mathrm{m}} \tilde{u}\right)$ was determined for every hot-wire data point from a measured hot-wired voltage, mean static pressure and static temperature. Since fluctuations in density are small compared to fluctuations in velocity, the instantaneous velocity could be determined by dividing the $\rho_{\mathrm{m}} \tilde{u}$ product by the average flow density determined for the data-sampling period from measurements of static pressure and temperature to yield velocity:

$$
\tilde{u}=\rho_{\mathrm{m}} \tilde{u} / \rho
$$

Figure 2 shows a sample blowdown calibration of the hot-wire. Also included in the plot is data taken from a small blowdown wind tunnel 
used for calibration of various probes for use in compressible flows. The data from the small wind tunnel spans a larger Reynolds number range than that achievable during blowdown calibration. The data from the two different tunnels are in good agreement.

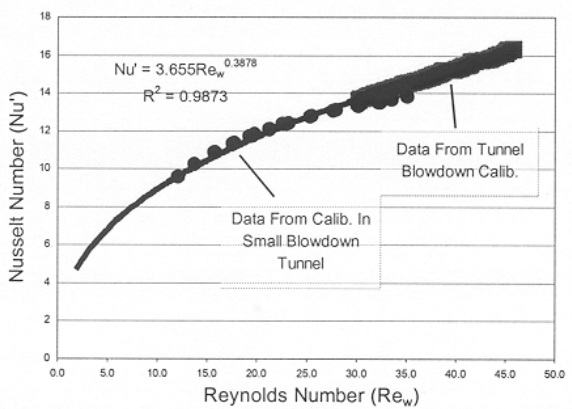

Figure 2. Sample hot-wire blowdown calibration curve

\section{Data Acquisition and Reduction}

To characterize the turbulence from the turbulence grid, calibration of the hot-wire was necessary, and statistical and spectra analyses were performed on the time-resolved velocity data. For low speed tests, the data were sampled at $20 \mathrm{kHz}$, filtered at $10 \mathrm{kHz}$ and a $5 \mathrm{~V}$ DC offset was applied to increase dynamic resolution. All data points were sampled for 6 seconds and four samples were taken at each point for averaging purposes. For the transonic facility testing, the data was sampled at $100 \mathrm{kHz}$ and filtered at $40 \mathrm{kHz}$, providing shorter sampling periods of approximately 1.3 seconds. The DC offset was adjusted to account for higher flow velocity to provide better dynamic resolution of the signal.

Statistical and spectral analyses included calculation of the mean velocity $(U)$, fluctuating component of velocity $(u)$, root mean square (RMS) of the fluctuating component of velocity $\left(u^{\prime}\right)$, autocorrelation $\left(R_{11}\right)$, probability density functions (PDF), and power spectral density (PSD).

The streamwise instantaneous velocity signal $(\tilde{u})$ can be decomposed into a mean and fluctuating component as follows:

$$
\tilde{u}=U+u
$$

where $\mathrm{U}$ is the mean velocity and $\mathrm{u}$ is the fluctuating component of velocity. The mean, fluctuating component and RMS of the discretized velocity data were calculated according to the equations in the nomenclature section. Using these values, the turbulence intensity $\mathrm{Tu}$ of the flow at a given point was calculated by:

$$
T u=\frac{u^{\prime}}{U}
$$

The autocorrelation $\left(\mathrm{R}_{11}\right)$ of the fluctuating component of velocity (normalized such that $R_{11}$ is equal to 1 at zero lag, $\tau=0$ ), was calculated by:

$$
R_{11}(\tau)=\left(\overline{\frac{u(t) \cdot u(t+\tau)}{u^{\prime 2}}}\right)=\frac{1}{N}\left(\frac{\sum_{i=1}^{N} u_{i} \cdot u_{i+j}}{u^{\prime 2}}\right)
$$

$$
\text { where } \tau=j \cdot \Delta t
$$

The autocorrelation was used to determine the integral time scale $(T)$ by integrating under the curve to the first zero crossing.

$$
T=\int_{t=0}^{\infty} R_{11}(\tau) d \tau=\sum_{i=1}^{N_{0}} R_{11 i} \cdot \Delta \tau
$$

where $\mathrm{N}_{0}$ is the point of the first zero crossing

The streamwise integral length scale, which is representative of the largest eddies in the turbulent flowfield, was then determined by invoking Taylor's hypothesis of frozen turbulence:

$$
\Lambda_{x}=\mathrm{U} \cdot T
$$

The power spectral density (PSD) of each data set was determined and frequency averaged to give a cleaner curve by averaging PSD data over a frequency range of $\Delta \mathrm{f}=100 \mathrm{~Hz}$. The power spectral density gives an indication of turbulent energy content of the flow at different frequencies, which are inversely proportional to the size of the turbulent eddies in the flow (the wave number, $\kappa$, is equal to $2 \pi \mathrm{f} / \mathrm{U})$. The PSD was also used to check for vortex shedding from the bars. The PSD data was normalized and compared to the theoretical Von Kármán spectrum equation [21]:

$$
\frac{E(f) \cdot U}{u^{\prime 2} \cdot \Lambda_{x}}=4 \cdot\left(1+\left(\frac{8 \pi f \cdot \Lambda_{x}}{3 \cdot U}\right)^{2}\right)^{-5 / 6}
$$

Figure 3 shows an example of measured turbulence data compared to the Von Kármán equation.

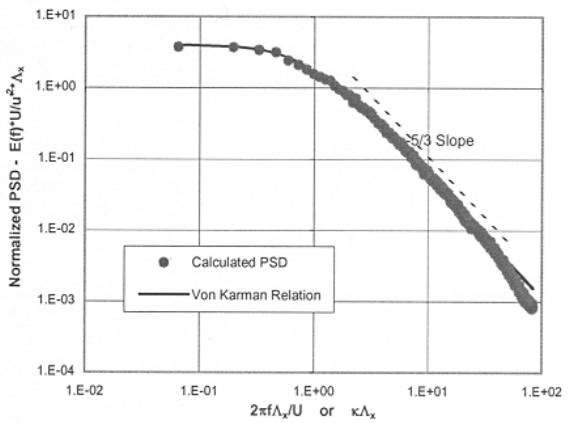

Figure 3. Low speed turbulence PSD compared with Von Kármán equation

Using these statistical and spectral analysis tools, the characteristics of the turbulence could be determined.

\section{Uncertainty Analysis}

Precision uncertainty of measurements of velocity was determined using the computerized uncertainty analysis method outlined in Moffat [22]. Uncertainty in the hot-wire velocity measurements in the low speed facility was estimated to be $\pm 1.5 \%$. Uncertainties in turbulence intensity and length scale in the low speed facility were $\pm 2 \%$ and $\pm 6 \%$, respectively. 
Uncertainty in velocity measurements with the hot-wire in the transonic facility was estimated to be $\pm 2.3 \%$. Uncertainties in turbulence intensity and length scale in the transonic facility were $\pm 3.2 \%$ and $\pm 6 \%$, respectively.

\section{LOW SPEED TESTING OF TURBULENCE GRID}

As mentioned previously, initial testing of the grid was performed in a low speed wind tunnel. The initial testing served two purposes First, testing was done to benchmark the turbulence measurement methodology. Second, results were used to obtain baseline numbers to determine what levels of turbulence to expect in the transonic facility and for comparison of turbulence intensity and length scale in two different flow fields.

The initial design of the turbulence grid was based on a combination of results from previous grid generated turbulence work. Simulation of turbulence with a mock combustor or jets in crossflow were not considered, since it was desired to employ a simplistic method. The initial grid design for this work was an actively blown turbulence generating grid incorporating both upstream and downstream blowing. The design was based primarily on work by Radomsky and Thole [12] and by Giel [15]. Both of these studies successfully generated large-scale, high intensity turbulence with actively blown turbulence grids in low and high-speed flow facilities, respectively.

The design that was studied for the present work consisted of three $2.54 \mathrm{~cm}$ ( 1 in.) hollow, square bars spaced $7.62 \mathrm{~cm}$ ( 3 in.) apart between bar edges with no vertical bars. Each bar has nine $3.2 \mathrm{~mm}$ (1/8 in.) holes spaced $2.54 \mathrm{~cm}$ ( 1 in.) between centers on both the upstream and downstream sides of the bars, allowing for blowing of high-pressure air in both directions. The upstream blowing was performed to increase shear stresses upstream of the grid to produce high turbulence levels. Downstream blowing was performed to fill in the wake areas behind the bars to create better flow uniformity entering the cascade.

Mean and root-mean-square (RMS) velocity measurements were taken at various locations throughout the test section. Flow uniformity was also checked (if wakes are present from bars on grid) in the test section. This traverse was performed at 13 bar widths downstream of the grid. Flow uniformity at this location was shown to be $\pm 2.5 \%$.

Four locations in the streamwise direction downstream of the grid were chosen to take data in the center of the passage (behind the center bar) to calculate the streamwise variation of the turbulence intensity and length scale. These locations are detailed in Table 1.

Table 1: Low Speed Testing Data Acquisition Locations

\begin{tabular}{||c|c||}
\hline \hline Location \# & $\begin{array}{c}\text { Distance Downstream of Grid } \\
(\mathrm{x} / \mathrm{d})\end{array}$ \\
\hline \hline 1 & 9.5 \\
\hline 2 & 13.5 \\
\hline 3 & 17.5 \\
\hline 4 & 21.5 \\
\hline
\end{tabular}

The mass flow ejected from the grid holes was adjusted in an attempt to reach the turbulence intensity and length scale desired. Momentum flux ratio (I) was believed to be the blowing parameter to match since turbulence is generated from shear stresses, which balance with momentum. Figure 4 - Figure 6 plot the mean velocity, turbulence intensity and length scale versus the blowing rate (as momentum flux ratio). The maximum value of blowing, if plotted as mass flux ratio (M) would be approximately a mass flux ratio of 14 . At a momentum flux ratio of 70 , the mass flux ratio is approximately
9. The mass flux ratio is, of course, not linear with momentum flux ratio. Of primary importance was the turbulence intensity and length scale, decay of turbulence, flow uniformity and the effect of active blowing. When used in passive mode (i.e. without blowing activated) the grid produced turbulence with intensity of $17 \%$ at a distance of 9.5 bar widths downstream of the grid (Location 1), which decayed to approximately $10 \%$ at a streamwise distance of 21.5 bar widths (Location 4), with length scales of approximately $2 \mathrm{~cm}$.

The mean flow in the test section without the grid installed was approximately $20 \mathrm{~m} / \mathrm{s}$ at center passage, which is independent of streamwise measurement location. With the grid installed, Figure 4 demonstrates that at locations near the grid without blowing, there is a velocity deficit due to the wake of the grid bars. This velocity deficit decreases in the streamwise direction and away from the influence of the bars. With blowing activated, the mean velocity in the test section will increase due to addition of mass flow from the blowing bars. Based on holding the inlet velocity fixed, a conservation of mass analysis predicts the expected increase to be from $20 \mathrm{~m} / \mathrm{s}$ with no blowing to $23 \mathrm{~m} / \mathrm{s}$ at the highest momentum flux ratio. Another trend that can be seen from the data is the effect of the bar wake. Recall that the measurements shown in Figure 4 are taken directly behind one of the bars (cross-stream surveys were not taken). At low momentum flux ratios there is clearly a reduced velocity at the first position, which is actually most evident at a momentum flux ratio of approximately $\mathrm{I}=70$. Even though there is blowing from the bars at this momentum flux ratio, it is not enough to fill in the bar wakes and yet there is enough upstream blowing to appear as a large blockage for the approaching flow. As a result of this flow blockage, the velocity deficit of the wake appears even greater. At the highest momentum flux ratio the data show there is a fairly constant velocity as the flow progresses downstream, indicating the mean flowfield is likely uniform. The decrease in mean velocity at the highest blowing rate is due to the plenum pressure dropping, most likely due to the inlet flow to the plenum choking.

The turbulence intensity of the flow without the grid installed was approximately $2 \%$ upstream of the grid. Figure 5 demonstrates that with the grid in place without blowing, the intensity increases significantly to approximately $10-15 \%$ depending on streamwise location. Blowing was used to increase turbulence intensity and create larger length scales. As blowing is activated and increased, there is first a decrease in turbulence intensity, then a steady increase with blowing rate. The initial decrease is due to filling in the wake of the bars, which essentially reduces the effectiveness (or effective bar width) of the grid. This is in agreement with results observed by Boyle [14] where active blowing at low blowing ratios is seen to slightly decrease turbulence intensity.

The integral length scale of the flow was shown, with the exception of one point, to steadily increase with blowing rate as can be seen in Figure 6. The length scale at higher blowing rates is seen to settle out with no further increase. The results show that at approximately 18 bar widths downstream of the grid, the turbulence intensity is as high as $17 \%$ with an integral length scale of $3.5 \mathrm{~cm}$. This corresponds to $\Lambda_{\mathrm{x}} / \mathrm{c}=0.26$ based on the blade chord length in the transonic facility. 


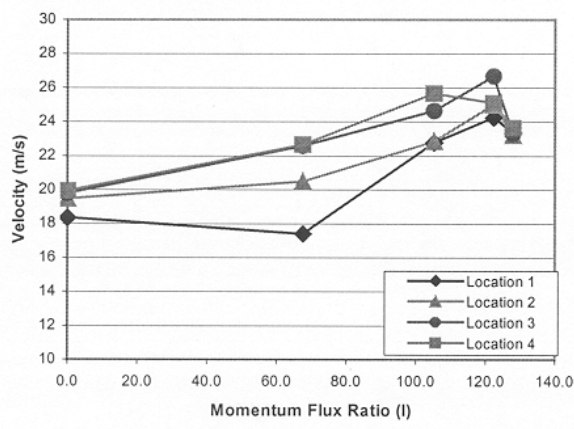

Figure 4. Plot of mean velocity at 4 streamwise locations at different blowing rates

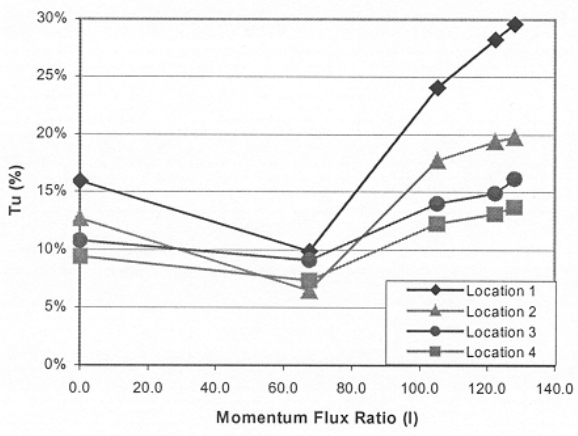

Figure 5. Plot of turbulence intensity at 4 streamwise locations at different blowing rates

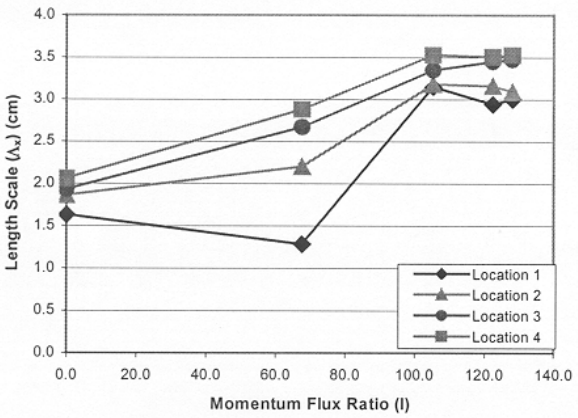

Figure 6. Plot of integral length scale at 4 streamwise locations at different blowing rates

Although the grid only consisted of horizontal bars, which are large compared to the size of the test section, the decay of turbulence intensity without blowing was shown to agree well with published correlations (Baines and Peterson [23]). The decay of turbulence intensity and dispersion of length-scale can be determined by:

$$
T u=c \cdot\left(\frac{x}{d}\right)^{n} \quad \text { and } \quad \Lambda_{x}=a \cdot d \cdot\left(\frac{x}{d}\right)^{m}
$$

where $\mathrm{c}$ and a are constants given as 0.80 and 0.20 , respectively, $\mathrm{x}$ is the streamwise distance, $\mathrm{d}$ is the bar width, and $\mathrm{n}$ and $\mathrm{m}$ are exponents. The value of $\mathrm{n}$ is typically $-5 / 7$ and $\mathrm{m}$ is between 0.5 and 0.56 according to the published data.

Figure 7 and Figure 8 demonstrate the variation of turbulence intensity and length scale in the streamwise direction, respectively. Results are presented for both the low-speed and high-speed testing of the grid, although the high-speed results will be discussed later in this work. It can be seen in Figure 7 that the low speed turbulence intensity data without blowing agree very well with the published correlations. The decay data with blowing is seen to deviate from this correlation, with greater deviation with higher blowing ratio Dispersion of length scale does not agree as well with the correlation.

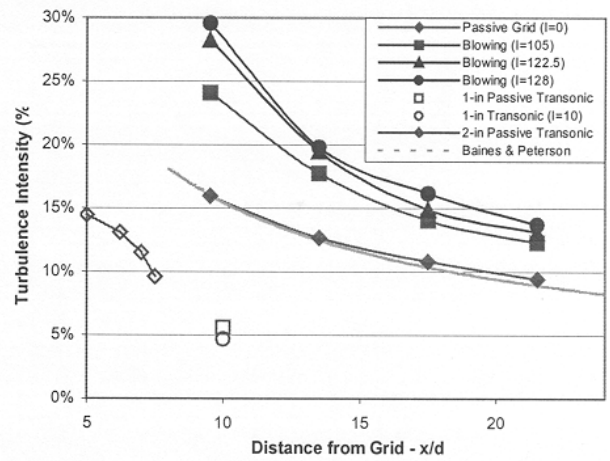

Figure 7. Variation of turbulence intensity in the streamwise direction with and without blowing

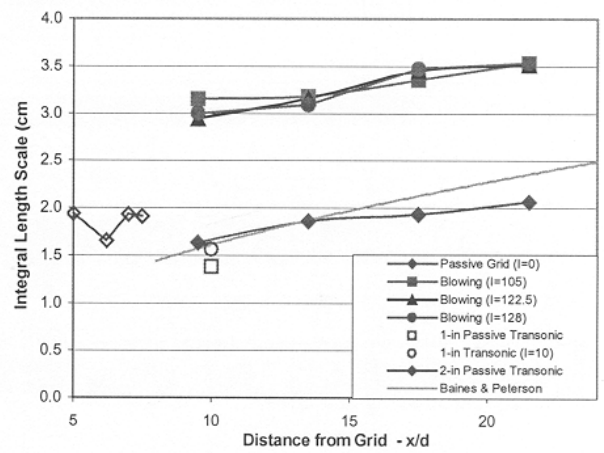

Figure 8. Variation length scale in the streamwise direction with and without blowing 


\section{TESTING OF TURBULENCE GRID IN TRANSONIC FACILITY}

With initial testing of the grid in the low speed facility providing satisfactory results, the initial grid design was moved to the high-speed facility for testing. Hot-wire data were taken at streamwise locations near those taken in the low speed facility. Data acquisition locations for the transonic facility are illustrated in Figure 9. The locations are shown as T0-T4 to avoid confusion with the locations in the low-speed testing.

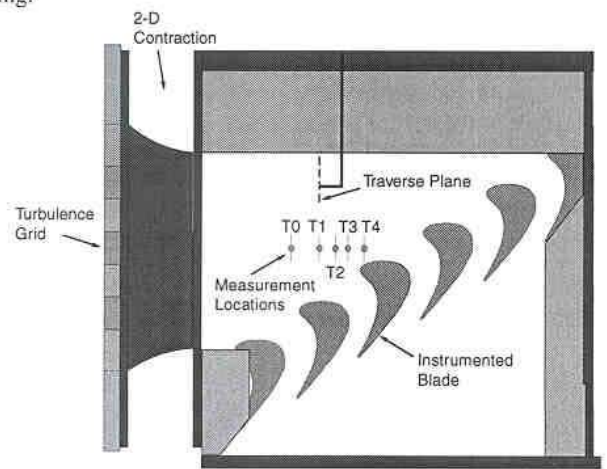

Figure 9. Hot-wire measurement locations in transonic facility

The turbulence grid was initially operated in passive mode in the transonic facility. In passive mode, the turbulence results from the grid did not match the levels measured in the low speed facility, generating test section inlet (location T0) turbulence intensity of only $5 \%$ as compared to approximately $12 \%$ in the low speed facility, which would decay to even lower levels before reaching the leading edge of the turbine blade cascade. Matching active blowing to the level achieved in the low speed facility was not possible, since matching momentum flux ratio of the blowing would require bar plenum blowing pressures exceeding the levels achievable within the transonic facility. It was determined that blowing pressures approaching 600-700 PSIG would be needed to match bar blowing momentum flux ratio with the low speed tests due to the high velocity and higher density in the freestream. Operating in passive mode (i.e. no blowing), the grid open area was $80 \%$ and the bar Reynolds number was much higher than in the low speed test rig (approximately 8 times higher), thus the flow experienced smaller effective blockage and lower turbulence levels when compared to the low speed tests.

In an effort to achieve more desirable turbulence levels in the transonic facility, the turbulence generator was modified by increasing the bar width to $5.08 \mathrm{~cm}(2 \mathrm{in}$.) in order to decrease the open area of the grid to $60 \%$. This modification was made based on results achieved by Polanka and Bogard [13] using large bars. This modified grid, operated in passive mode, generated cascade inlet turbulence levels approaching $15 \%$, with an integral length-scale of $2 \mathrm{~cm}$ Blowing was performed with the new grid; however, the turbulence intensity decreased, which is consistent with results reported by Boyle [14]. Blowing at low pressure fills in the wake and thus reduces turbulence intensity. Also, the upstream blowing at low pressure does not generate high turbulence intensity. Again, in order for blowing to have a positive effect on turbulence generation, blowing pressures of higher than what is achievable in the transonic facility are needed.

Measurements of the streamwise decay of turbulence were performed to determine the variation in both turbulence intensity and length scale as the turbulent flow progresses from the inlet of the cascade toward the entrance to the cascade blade passages. These tests were performed with a coarse spacing (only 4 locations) but demonstrated that the turbulence intensity decays to approximately 10 $12 \%$ approaching the entrance to the cascade passages (location T4). This data is included in Figure 8 and Figure 9. The turbulence decay did not match well with grid generated turbulence decay correlations. The strong decay in turbulence intensity may be due to non-uniform turbulence in the cross-stream direction, or may be a result of effects from the blade (i.e. local velocity is higher due to acceleration of the flow on the suction surface of the blade). Future experiments will be performed to measure cross-stream variation in turbulence intensity and further streamwise decay of turbulence. The length scale of the turbulence does not grow to any extent in the short distance measured, with integral length-scales of approximately $2 \mathrm{~cm}\left(\Lambda_{x} / \mathrm{c}=0.15\right)$ being measured at different streamwise locations. Turbulence parameters for different grid designs (with and without blowing) and measurement locations, including selected data from the low speed testing, are shown in Table 2 below.

Table 2: Turbulence Generation Results

\begin{tabular}{|c|c|c|c|c|c|c|c|}
\hline & \multirow[b]{2}{*}{$\begin{array}{l}\text { Location } \\
\text { Number }\end{array}$} & \multirow[b]{2}{*}{$\begin{array}{c}\text { Blowing } \\
\text { Rate } \\
\text { (1) } \\
\end{array}$} & \multicolumn{2}{|c|}{$\begin{array}{c}\text { Distance from } \\
\text { Grid }\end{array}$} & \multirow{2}{*}{$\begin{array}{c}\begin{array}{c}\text { Distance } \\
\text { from }\end{array} \\
\text { LE of } \\
\text { Instr Blade } \\
\quad(x / c) \\
\end{array}$} & \multirow{2}{*}{$\begin{array}{c}\text { Turbulence } \\
\text { Intensity } \\
T u \\
(\%) \\
\end{array}$} & \multirow{2}{*}{$\begin{array}{c}\text { Length } \\
\text { Scale } \\
\mathrm{N}_{1} \\
(\mathrm{~cm}) \\
\end{array}$} \\
\hline & & & $\begin{array}{c}\text { Original } \\
\text { Grid } \\
x / d_{1} \\
\end{array}$ & $\begin{array}{c}\text { Modified } \\
\text { Grid } \\
x / d_{2} \\
\end{array}$ & & & \\
\hline Low Speed Facility & $\begin{array}{l}2 \\
2 \\
\end{array}$ & $\begin{array}{c}. \\
122 \\
\end{array}$ & $\begin{array}{r}13.5 \\
13.5 \\
\end{array}$ & . & . & $\begin{array}{r}13 \% \\
20 \% \\
\end{array}$ & $\begin{array}{r}2 \\
3.5 \\
\end{array}$ \\
\hline Transonic Factility & & & & & & & \\
\hline $1 \%(2.54 \mathrm{~cm})$ Bars & $\begin{array}{l}\text { To } \\
\text { TO }\end{array}$ & 10 & $\begin{array}{l}10 \\
10 \\
\end{array}$ & . & $\begin{array}{l}0.88 \\
0.88\end{array}$ & $\begin{array}{l}5.58 \% \\
4.69 \%\end{array}$ & $\begin{array}{l}1.38 \\
1.56\end{array}$ \\
\hline $2^{n}(5.08 \mathrm{~cm})$ Bars & $\begin{array}{l}\mathrm{TO} \\
\mathrm{T} 1 \\
\mathrm{~T} 3 \\
\mathrm{~T} 3 \\
\mathrm{~T} 4\end{array}$ & . & - & $\begin{array}{c}5 \\
6.2 \\
7 \\
7 \\
7.5\end{array}$ & $\begin{array}{l}0.88 \\
0.47 \\
0.14 \\
0.14 \\
0.05\end{array}$ & $\begin{array}{l}14.45 \% \\
13.08 \% \\
11.51 \% \\
10.62 \% \\
9.64 \%\end{array}$ & $\begin{array}{l}1.94 \\
1.65 \\
1.93 \\
2.32 \\
1.91\end{array}$ \\
\hline
\end{tabular}

Total pressure traverse measurements were performed behind the turbulence grid, approximately $10.2 \mathrm{~cm}$ (4 in.) upstream of the leading edge of the center cascade passage in order to measure flow uniformity behind the modified grid. Each traverse covered about half the passage in the pitchwise direction. The modified grid has an open area of $60 \%$, with $40 \%$ flow blockage area, and thus there was concern with inlet flow non-uniformity due to large bar wakes. Total pressure surveys demonstrate that although there is a significant drop in total pressure across the grid (approximately 7\%), the total pressure of the flow approaching the leading edge of the cascade is relatively uniform, with total pressure uniformity of $\pm 1 \%$. The main tunnel flow passes through a 2:1 area contraction downstream of the grid before entering the cascade test section, which is believed to help in merging and mixing of the relatively large bar wakes, thus increasing flow uniformity. Results of the total pressure traverse for both the $2.54 \mathrm{~cm}$ ( 1 in.) and $5.08 \mathrm{~cm}$ ( 2 in.) bar grid designs are shown in Figure 10. It can be seen that the total pressure with the $2.54 \mathrm{~cm}$ bars is nonuniform, with wakes present without blowing. With blowing, the total pressure traverse data shows that there is a jet from the bars. The data 
for the $5.08 \mathrm{~cm}$ bars with blowing shows that there is a smaller total pressure drop across the grid, but the flow uniformity is not greatly improved. Since active blowing actually lowered the turbulence intensity slightly, blowing was not used once the grid was implemented for wind tunnel heat transfer tests.

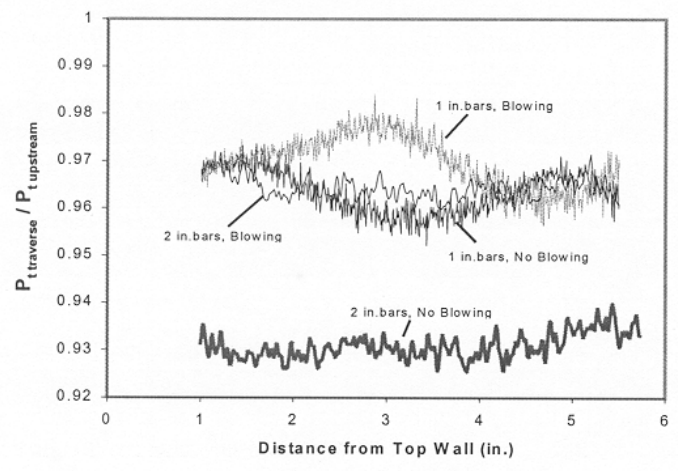

Figure 10. Total pressure uniformity downstream of turbulence grid

\section{CONCLUSIONS}

The development of a turbulence generator for use in a highspeed flow facility has been documented. The generator was tested in a low-speed environment with good results in turbulent flowfield and flow uniformity. Results from testing in a high-speed flowfield were in poor agreement with low-speed results due to higher Reynolds number (from higher velocity and density) providing inadequate flow blockage. The momentum flux ratio from low speed testing of the grid could not be matched in transonic facility. The grid was modified to create higher blockage and used as a passive grid. Results from the modified grid showed lower turbulence than originally desired, but with reasonably high intensity $(10-12 \%$ near the entrance to the cascade passages), large length scale $(2 \mathrm{~cm})$ compared to blade chord and good total pressure uniformity downstream of the grid near the entrance to the cascade passages.

\section{ACKNOWLEDGMENT}

This work was sponsored by the Air Force Office of Scientific Research (AFOSR), USAF, under grant/contract number F49620-011-0177, monitored by Dr. Tom Beutner. We would like to thank Paul Giel, Bob Boyle and Jim Van Fossen at NASA Glenn for their help and insight into developing the turbulence generator.

\section{REFERENCES}

[1] Van Fossen, 2000, Personal Communication, NASA Glenn Research Center. Research paper to be published.

[2] Moss, R.W. and Oldfield, M.L.G., 1991, "Measurements of Hot Combustor Turbulence Spectra," ASME 91-GT-351.

[3] Ames, F.E., 1997, "Aspects of Vane Film Cooling with High Turbulence - Parts I and II," ASME 97-GT-239/240.

[4] Wang, H.P., Goldstein, J., and Olson, R.J., 1998, "Effect of High Freestream Turbulence with Large Scale on Blade Heat/Mass Transfer," ASME 98-GT-107.

[5] Schauer, J.J and Pestian, D.J., 1996, "Film Cooling Heat Transfer with High Freestream Turbulence," ASME 96-WA/HT-6.
[6] Bons, J.P., MacArthur, C.A. and Rivir, R.B., 1996, "The Effect of High Free-Stream Turbulence on Film Cooling Effectiveness," ASME Journal of Turbomachinery, Vol. 118, pp. 814-825.

[7] Thole, K.A., Bogard, D.G. and Whan-Tong, J.L., 1994 , "Generating High Freestream Turbulence Levels," Experiments in Fluids 17, 375-380.

[8] Harrington, M.K., McWaters, M.A., Bogard, D.G., Lemmon, C.A., and Thole, K.A., 2001, "Full-Coverage Film Cooling with Short Normal Injection Holes," ASME 2001-GT-130.

[9] Barringer, M.D., Richard, O.T., Walter, J.P., Stitzel, S.M., and Thole, K.A., 2001, "Flowfield Simulations of a Gas Turbine Combustor," ASME 2001-GT-0170.

[10] Mehendale, A.B. and Han, J.C., 1992, "Influence of High Mainstream Turbulence on Leading Edge Film Cooling Heat Transfer," ASME Journal of Turbomachinery, Vol. 114, pp. 707715.

[11] Sauer, J., 1996, "The SUDI Turbulence Generator - A Method to Generate High Freestream Turbulence Levels and a Range of Length Scales," Thesis, University of Wisconsin/Universitat Karlsruhe

[12] Radomsky, R.W. and Thole K.A., 1998, "Effects of High Freestream Turbulence Levels and Length Scales on Stator Vane Heat Transfer," ASME 98-GT-236.

[13] Polanka, M.D., 1999, "Detailed Film Cooling Effectiveness and Three Component Velocity Field Measurements on a First Stage Turbine Vane Subject to High Freestream Turbulence," Ph.D. Dissertation, The University of Texas at Austin.

[14] Boyle, R. J., Lucci, B.L., Verhoff, V.G., Camperchioli, W.P. and La, H., 1998, "Aerodynamics of a Transitioning Turbine Stator Over a Range of Reynolds Numbers," ASME Paper 98-GT-295 (NASA/TM-1998-208408).

[15] Giel, P.W., Bunker, R.S., Van Fossen, G.J. and Boyle, R.J., 2000, "Heat Transfer Measurements and Predictions on a Power Generation Gas Turbine Blade," ASME 2000-GT-0209.

[16] Holmberg, D.G., 1996, "A Frequency Domain Analysis of Surface Heat Transfer/Freestream Turbulence Interactions in a Transonic Turbine Cascade," Ph.D. Dissertation, Virginia Tech.

[17] Nix, A.C., Diller, T.E. and Ng, W.F., 1997, "Experimental Evaluation of Heat Transfer Effects of Shock Waves on Transonic Turbine Blades," ASME 97-WA/HT-1.

[18] Nix, A.C., Reid, T., Peabody, H., Ng, W.F., Diller, T.E. and Schetz, J.A., 1997, "Effects of Shock Wave Passing on Turbine Blade Heat Transfer in a Transonic Cascade," AIAA-97-0160.

[19] Smith, D.E., Bubb, J.V., Popp, O., Grabowski, H.C., Diller, T.E., Schetz, J.A. and Ng, T.E., 2000, "Investigation of Heat Transfer in a Film Cooled Transonic Turbine Cascade, Part I: Steady Heat Transfer," ASME 2000-GT-202.

[20] Popp, O., Smith, D.E., Bubb, J.V., Grabowski, H.C., Diller, T.E. Schetz, J.A. and Ng, T.E., 2000, "Investigation of Heat Transfer in a Film Cooled Transonic Turbine Cascade, Part II: Unsteady Heat Transfer," ASME 2000-GT-203.

[21] Hinze, J.O., 1975, Turbulence, $2^{\text {nd }}$ Edition, McGraw-Hill, New York..

[22] Moffat, R.J., 1988, "Describing Uncertainties in Experimental Results," Exp. Thermal and Fluid Science 1988, 1:3-17.

[23] Baines, W.D. and Peterson, E.G., 1951, "An Investigation of Flow Through Screens," Trans. of the ASME, July 1951, pp. $467-$ 480. 


\section{High Intensity, Large Length-Scale Freestream Turbulence Generation in a Transonic Turbine Cascade}

A.C. Nix, A.C. Smith, T.E. Diller, W.F. Ng, K.A. Thole

Virginia Polytechnic Institute and State University

Blacksburg, VA 24061

Presented at 2002 IGTI Congress and Exposition, Amsterdam, NL

June 6, 2002

Paper No. GT-2002-30523

感

\section{Motivation}

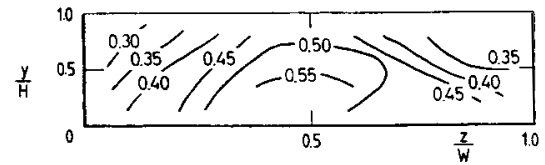

Goldstein, et al. (1983)

Early studies identified high levels of freestream turbulence exiting the combustor $\rightarrow$ Effects on heat transfer?

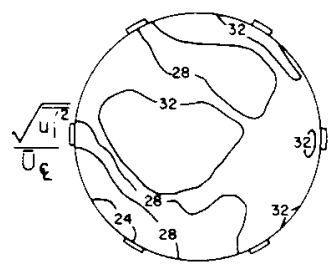

Kuotmos and McGuirk (1989)

感VirginiaTech 


\section{Objectives}

\section{The primary objectives of the current research effort are:}

- To experimentally study the effects of combustor exit freestream turbulence on turbine blade heat transfer

- Develop and test a turbulence grid to produce high-intensity, large-scale freestream turbulence in a transonic turbine cascade

䍐VirginiaTech

\section{Turbulence generating techniques}

\begin{tabular}{|c|c|c|c|c|c|}
\hline Investigator & $\begin{array}{c}\text { Turbulence } \\
\text { Level }\end{array}$ & $\begin{array}{l}\text { Length } \\
\mathrm{cm}\end{array}$ & $\begin{array}{l}\text { Scale } \\
\left(\Lambda_{\mathrm{x}} / \mathrm{c}\right)\end{array}$ & Method & $\begin{array}{c}\text { Flow } \\
\text { Condition }\end{array}$ \\
\hline $\begin{array}{l}\text { Mehendale and Han } \\
\text { (1992) }\end{array}$ & $13 \%$ & 3 & - & Blown Grid & Low speed \\
\hline Thole, et al. (1994) & $20 \%$ & $2-3$ & - & Jets in crossflow & Low speed \\
\hline Holmberg (1996) & $5 \%$ & 0.5 & $<0.1$ & Grid & Transonic \\
\hline $\begin{array}{l}\text { Schauer and } \\
\text { Pestian (1996) }\end{array}$ & $17 \%$ & 8 & - & Jets in crossflow & Low speed \\
\hline Ames (1997) & $13 \%$ & 1.6 & 0.11 & Mock combustor & Low speed \\
\hline Boyle, et al. (1998) & $10 \%$ & 1.5 & - & $\begin{array}{l}\text { Blown and unblown } \\
\text { grid }\end{array}$ & Low speed \\
\hline Wang, et al. (1998) & $18 \%$ & 8 & 0.43 & Mock combustor & Low speed \\
\hline $\begin{array}{l}\text { Radomsky and } \\
\text { Thole (1999) }\end{array}$ & $20 \%$ & $5-6$ & - & Blown bar array & Low speed \\
\hline $\begin{array}{l}\text { Polanka and } \\
\text { Bogard (1999) }\end{array}$ & $20 \%$ & 5 & - & $\begin{array}{l}\text { Passive bar array } \\
\text { (large blockage) }\end{array}$ & Low speed \\
\hline Giel, et al. (2000) & $10 \%$ & 2.2 & 0.17 & Active grid & Transonic \\
\hline $\begin{array}{l}\text { Barringer, et al. } \\
\text { (2001) }\end{array}$ & $18 \%$ & $6-7$ & 0.11 & $\begin{array}{l}\text { Combustor } \\
\text { simulator with large } \\
\text { jets }\end{array}$ & Low speed \\
\hline
\end{tabular}

罗VirginiaTech 


\section{Current turbulence generator development}

- Initial design was an actively blown bar arrangement based on low-speed work by Radomsky and Thole (1998) and work in a transonic facility by Giel, et al. (2000)

- Modification of the initial design was performed based on work of Polanka and Bogard (1999)
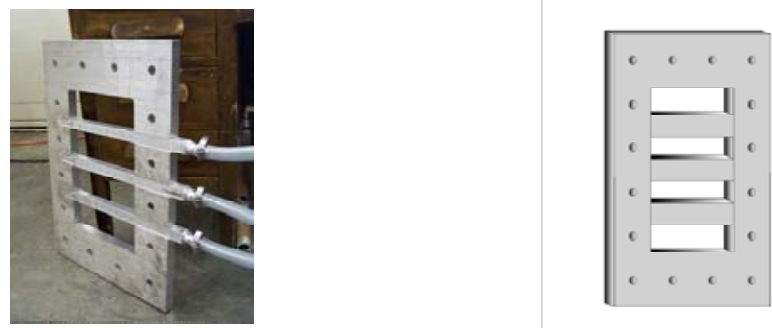

\section{忽VirginiaTech}

\section{Experimental Setup}

Two facilities were used to perform testing of the turbulence grid:

-Low-speed blower wind tunnel (inlet velocity $20 \mathrm{~m} / \mathrm{s}$ )

-Transonic cascade facility

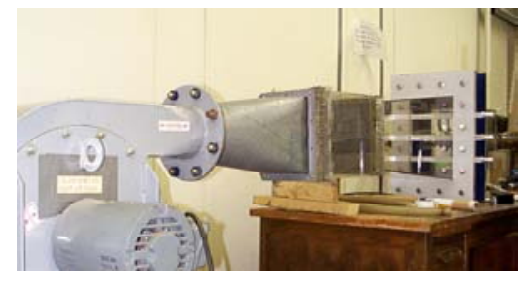
(inlet Mach number of $\mathrm{M}=0.4$, approximately $120 \mathrm{~m} / \mathrm{s}$ )

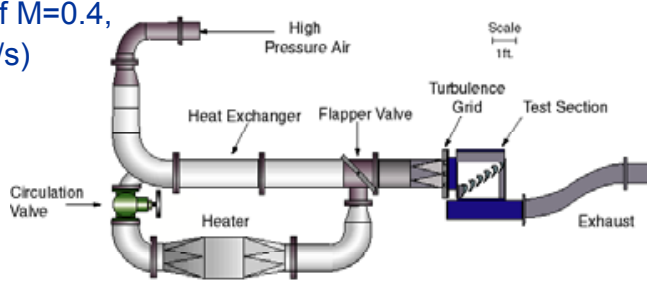




\section{Sample hot-wire calibration in the transonic facility}

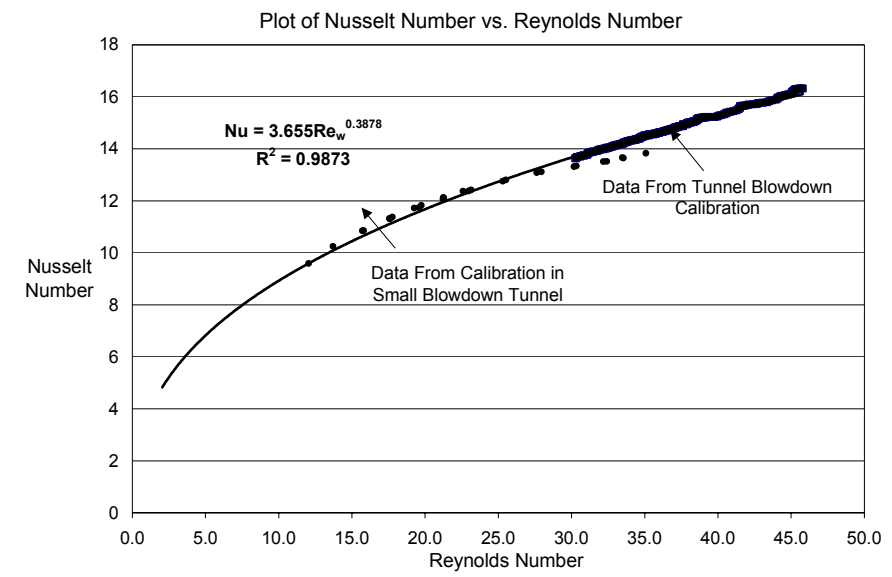

Measured values: $P_{t}, P_{s}, T_{t}$ and hot-wire voltage Hot-wire calibration shown as $\mathrm{Nu}=\mathrm{C}^{*} \mathrm{Re}^{\mathrm{x}}=\mathrm{V}^{2} / \mathrm{k}\left(\mathrm{T}_{\mathrm{m}}-\mathrm{T}_{\mathrm{s}}\right)$

\section{䍐VirginiaTech}

\section{Results of low-speed testing}

Freestream velocity as a function of momentum flux ratio and streamwise location

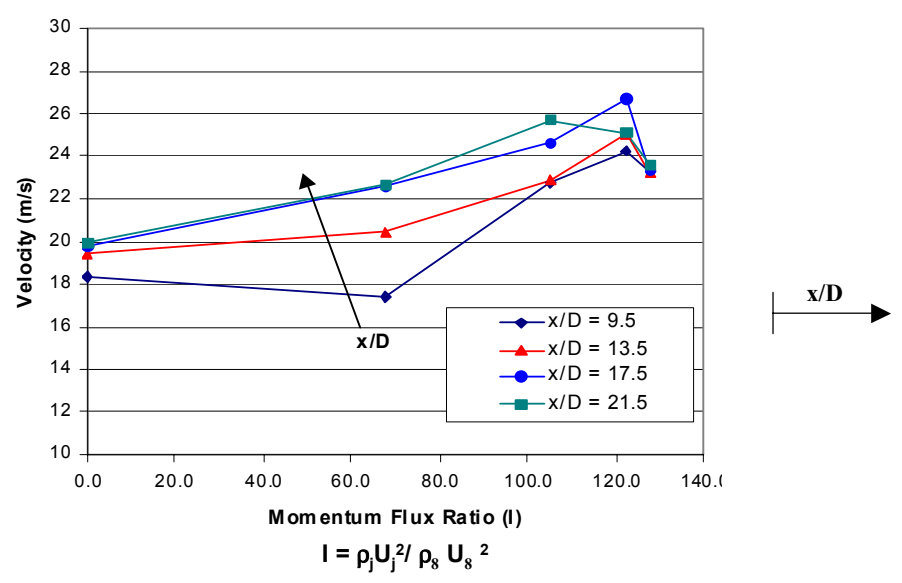

忽VirginiaTech 


\section{Results of low-speed testing}

Turbulence intensity as a function of momentum flux ratio and streamwise location

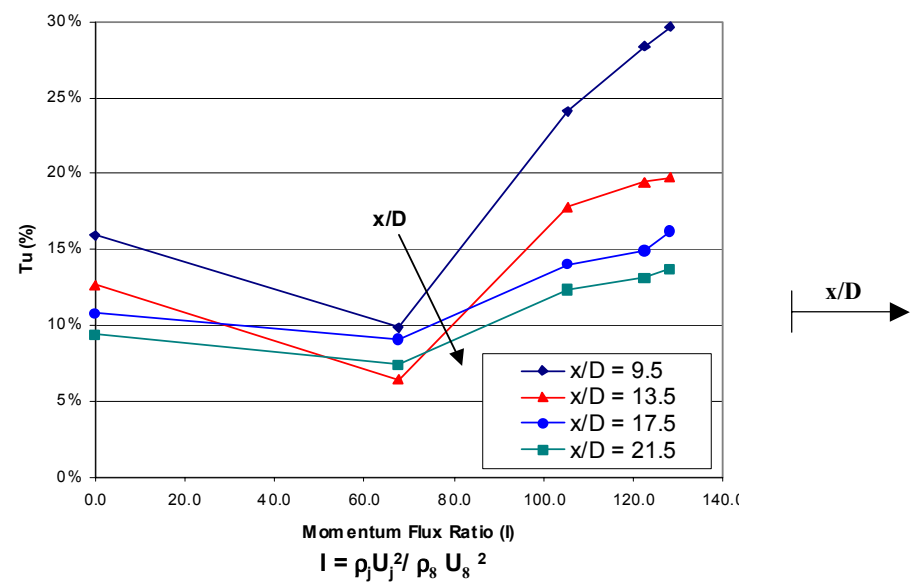

感VirginiaTech

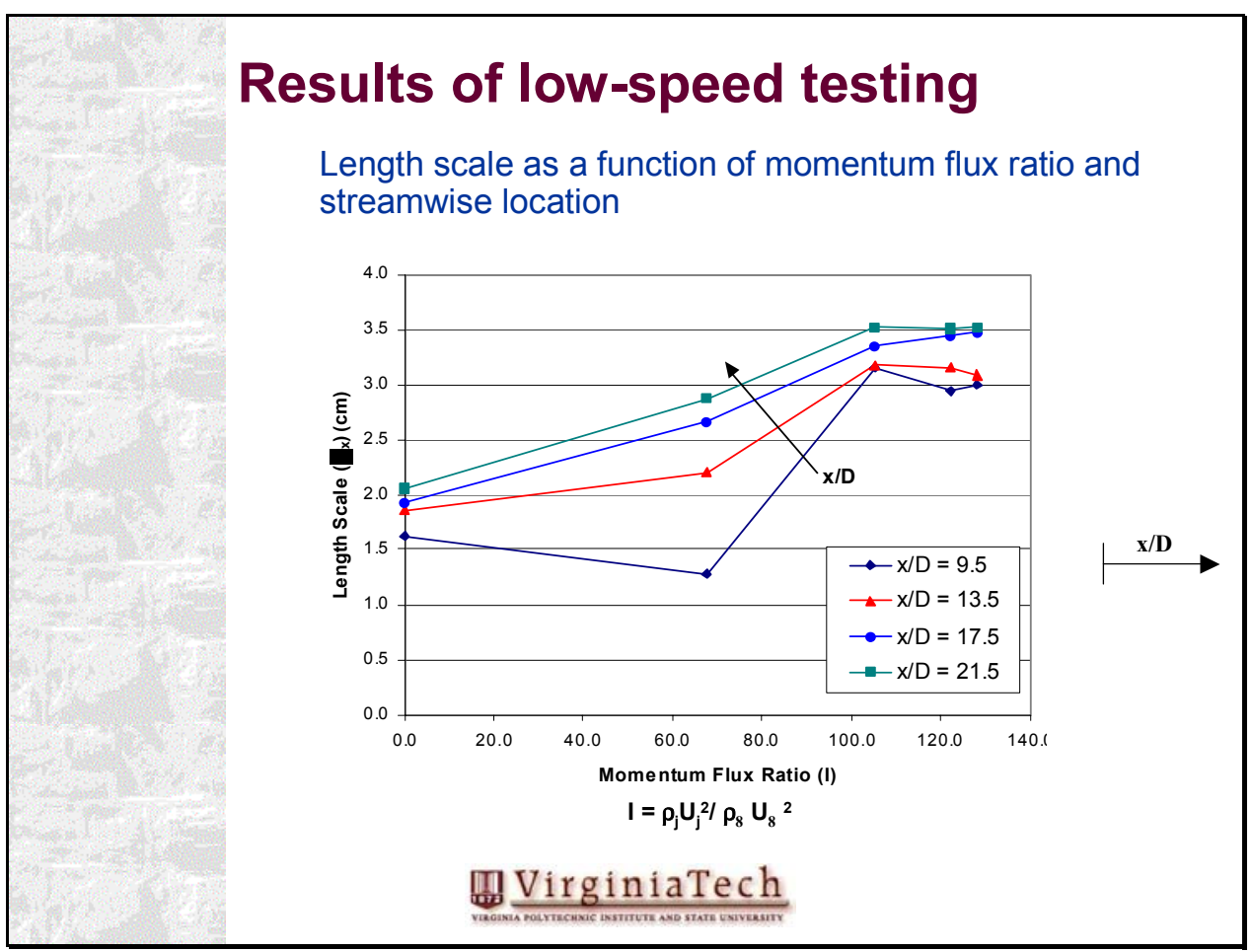




\section{Results of low-speed testing}

Streamwise variation in turbulence intensity

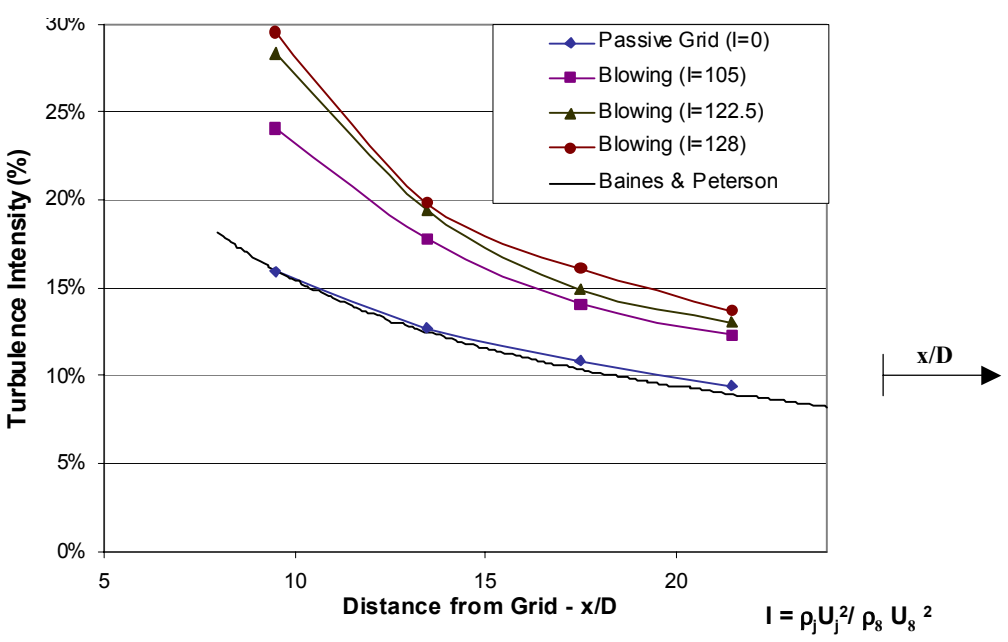

忽VirginiaTech

\section{Results of low-speed testing}

Streamwise variation in length scale

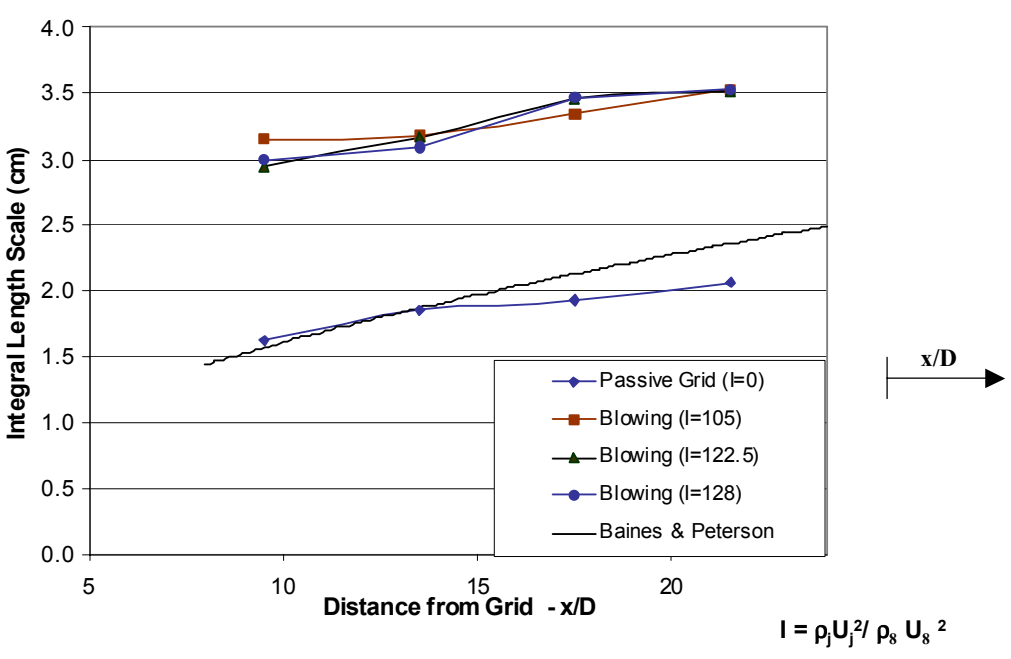

罗VirginiaTech 


\section{Testing in transonic facility}

Turbulence grid Pitchwise survey Streamwise survey

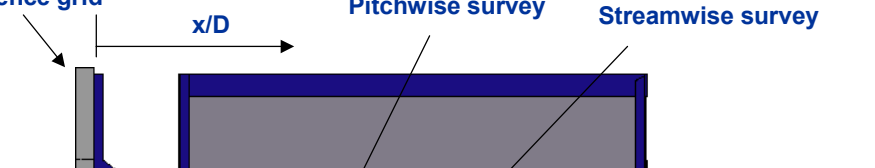

Cascade Design: Blade span (s) $-15.3 \mathrm{~cm}$ Aero. Chord (c) $-13.7 \mathrm{~cm}$ Pitch (p) $-12.2 \mathrm{~cm}$ $\mathrm{s} / \mathrm{c}-1.12$ $\mathrm{p} / \mathrm{c}-0.9$

Blade $1-x / D=7.25$ Blade $2-x / D=9.25$

$$
\text { 2-D }
$$

Cascade Test Section

Contraction

\section{䍐VirginiaTech}

\section{Results of Transonic Testing}

Comparison to Low Speed Testing

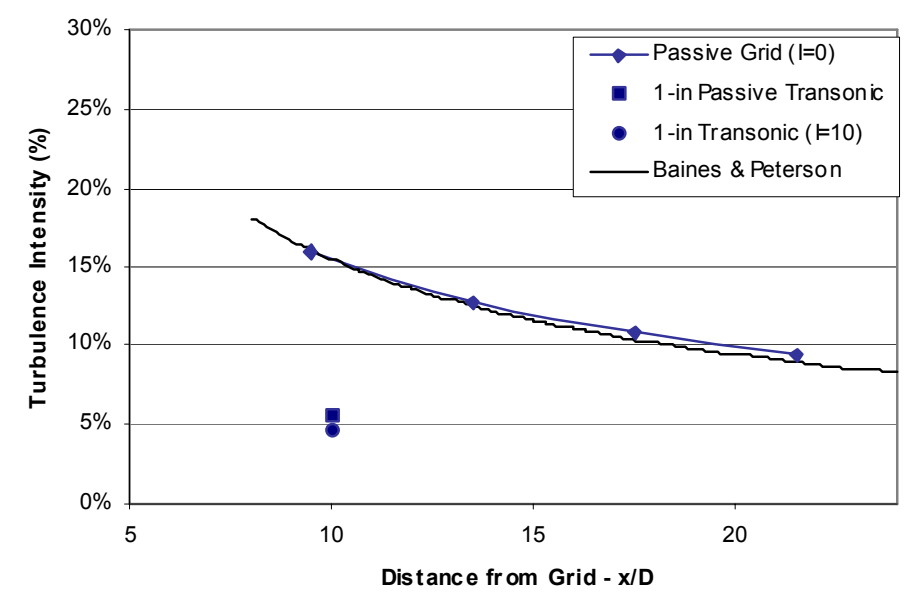

忽VirginiaTech 


\section{Grid Modification}

- Based on turbulence measurements with the initial grid design in the transonic facility, the grid was modified

- Modification of the initial design was performed based on work of Polanka and Bogard (1999) by increasing bar size to $5.08 \mathrm{~cm}$ (2 in)

- Turbulence measurements in the transonic facility were performed with the modified grid

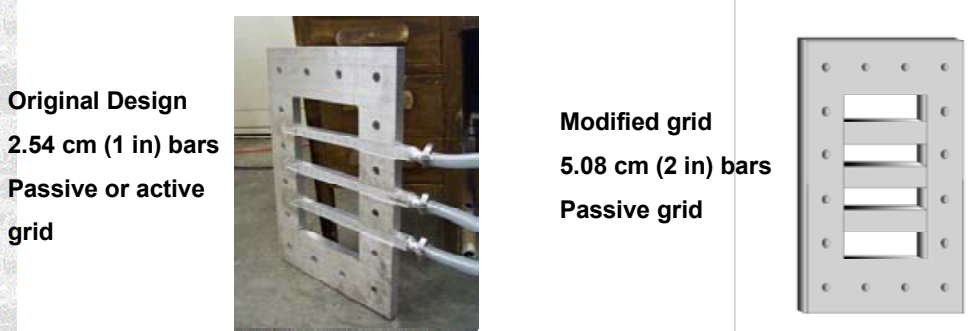

忽VirginiaTech

\section{Streamwise turbulence uniformity}

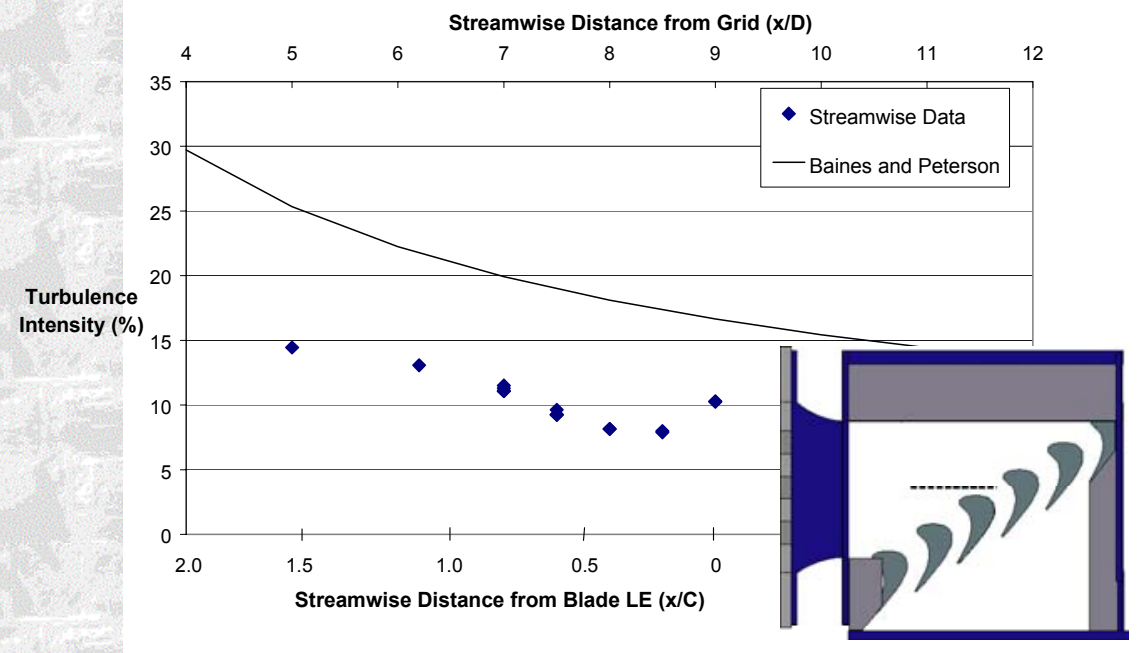

感VirginiaTech 


\section{Streamwise length scale uniformity}

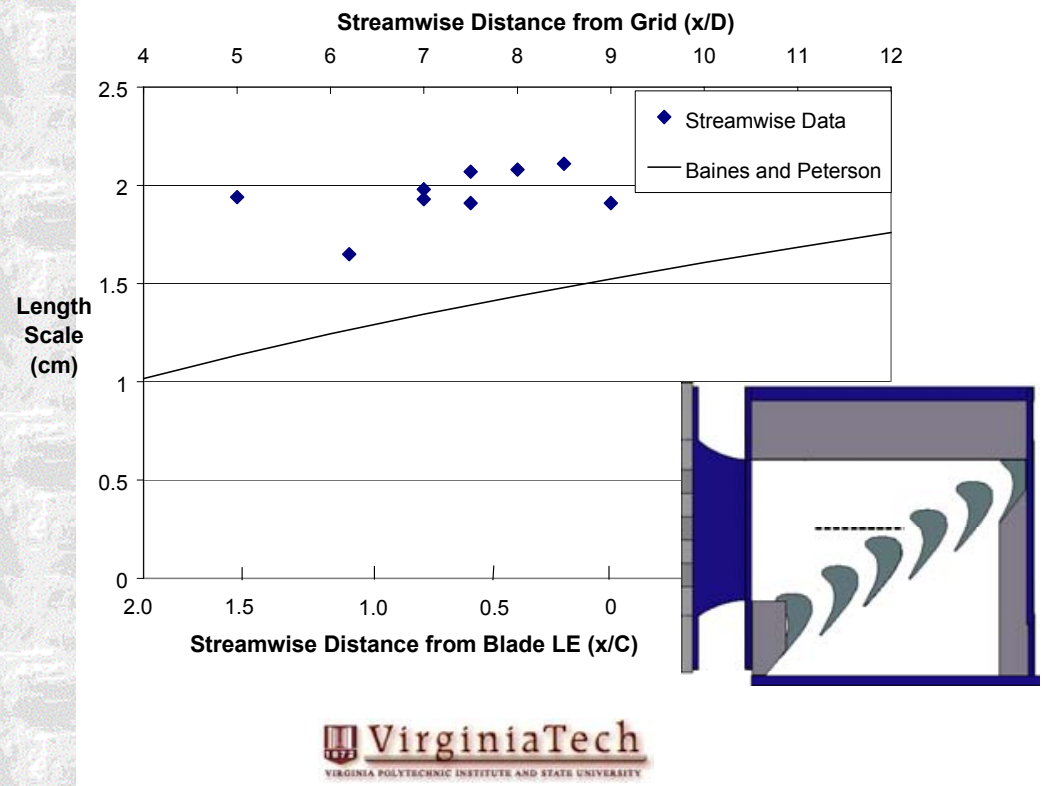

\section{Pitchwise survey}

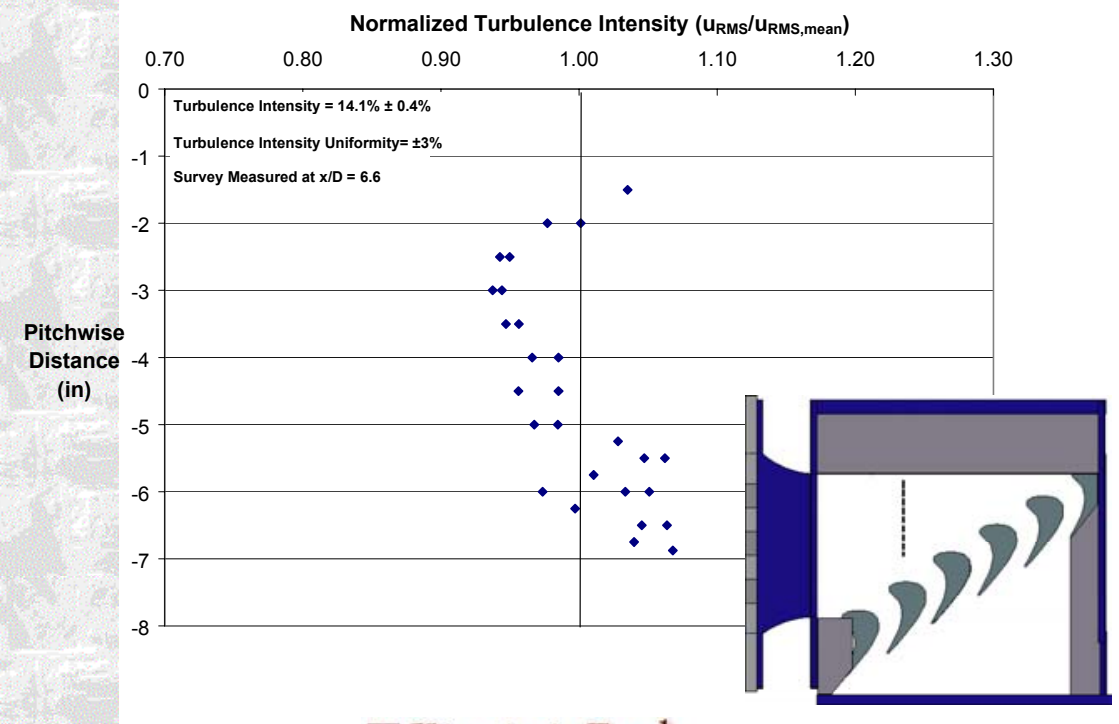

感VirginiaTech 


\section{Streamwise spectral data}

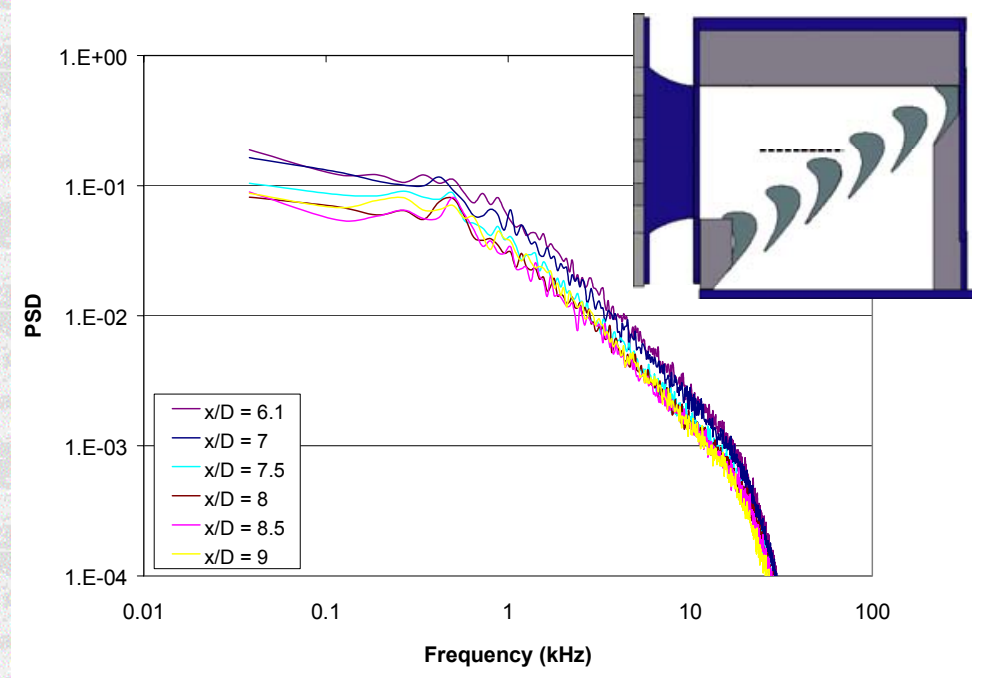

忽 VirginiaTech

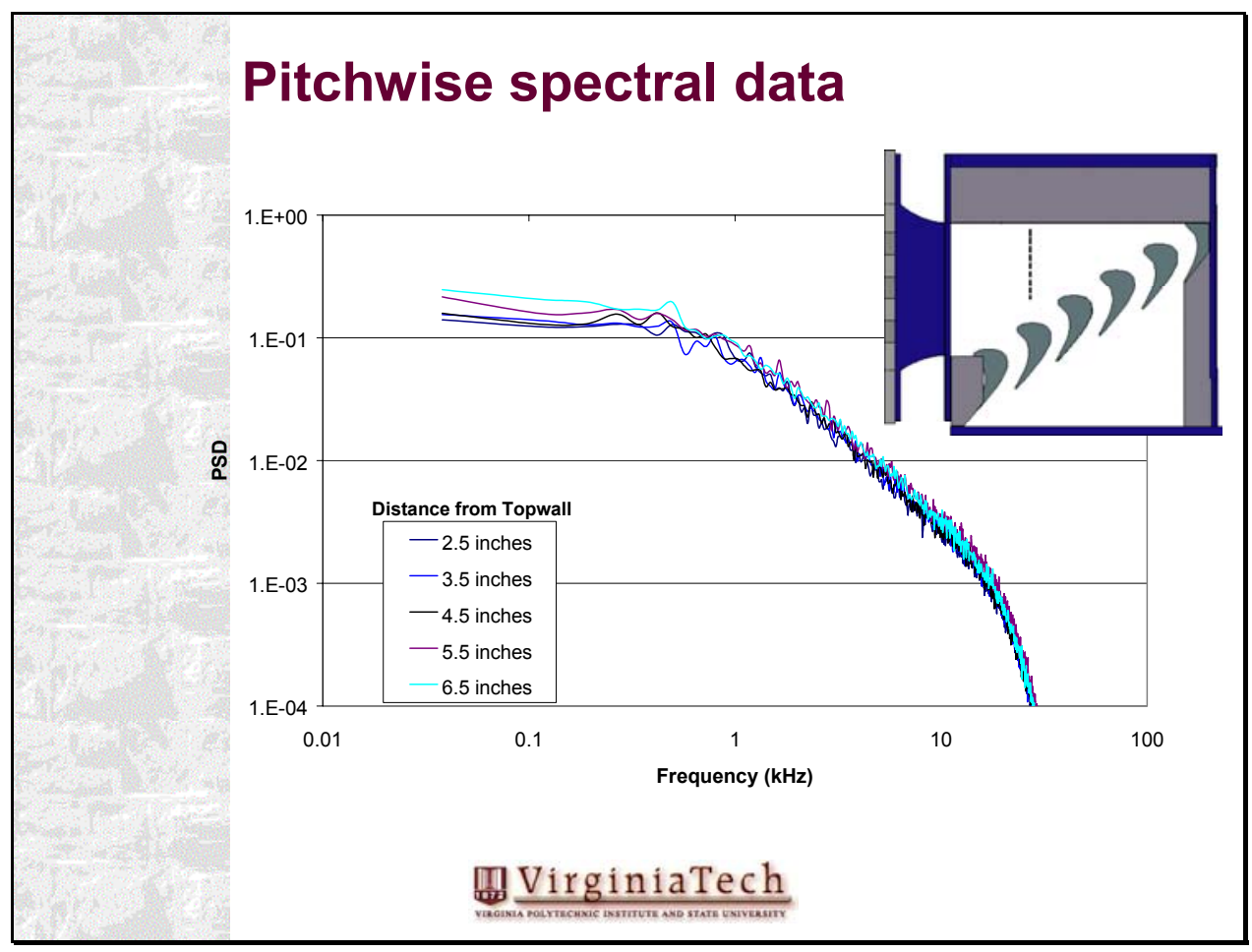




\section{Conclusions}

- The development of a turbulence generator for use in a transonic flow facility has been documented

- The generator was tested in a low-speed environment with good results in turbulent flowfield and flow uniformity

- Results from testing in a high-speed flowfield were in poor agreement with low-speed results

- The grid was modified to create higher blockage and used as a passive grid

- Modified grid generates 10-12\% turbulence near the entrance to the cascade passages with $2 \mathrm{~cm}$ length scale

-Spanwise and pitchwise uniformity of turbulence was shown to be good with no vortex shedding measured

MirginiaTech

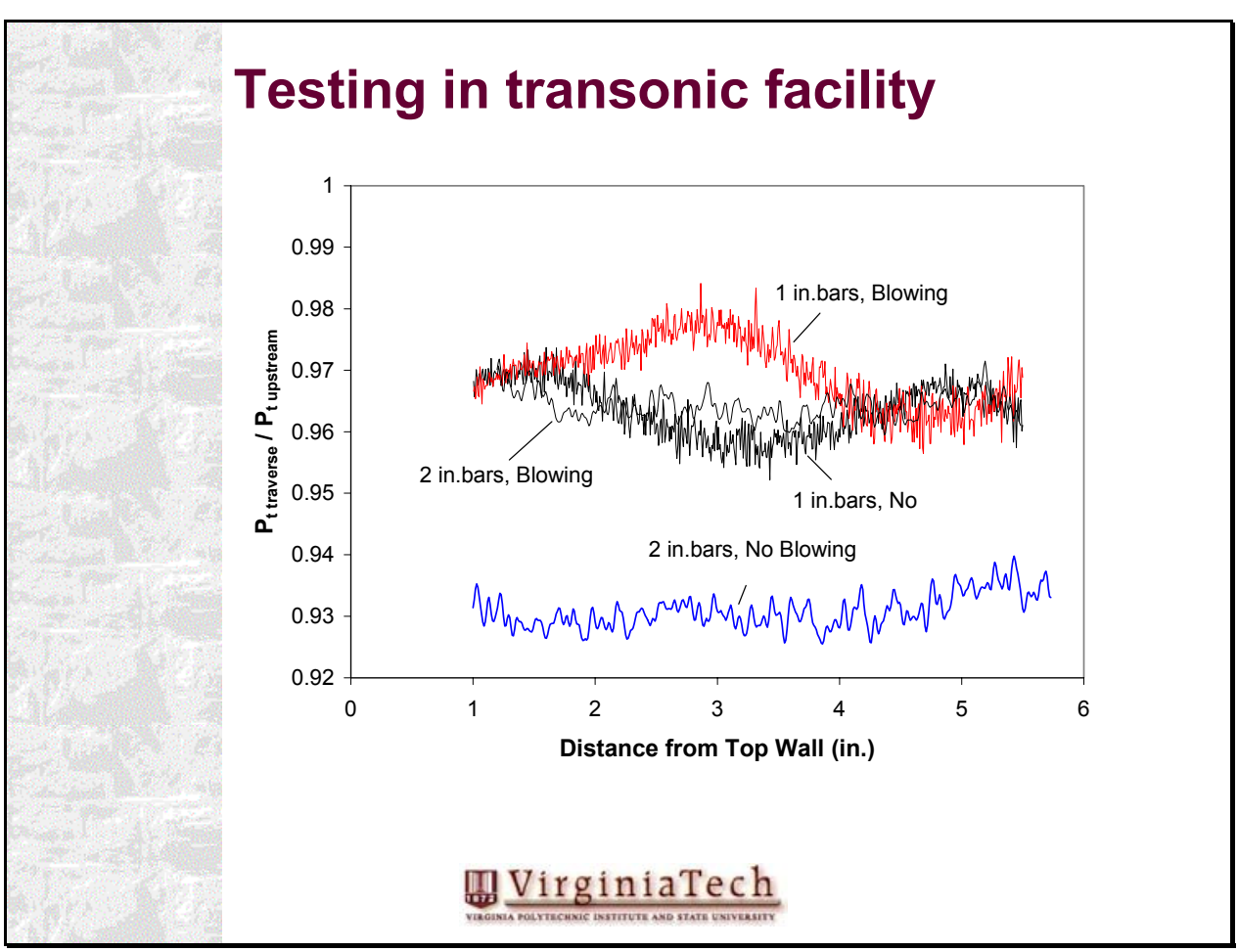




\section{APPENDIX B}

\section{MEASUREMENT UNCERTAINTY}

The following section documents measurement uncertainties of many of the reported data in this dissertation. Results presented in this work are dependent on experimental measurements, making determination of the uncertainty in these measurements important. The primary quantities of concern are the steady heat transfer coefficients, hot-wire velocity measurements (primarily $\mathrm{u}_{\mathrm{rms}}^{\prime}$ ), the integral length scale, and the model predicted values of increase in heat transfer. The uncertainty associated with each one of these measured and calculated values will be presented. The uncertainty of experimental measurements and values calculated from these measurements were determined using the computerized uncertainty analysis method (perturbation method) outlined in Moffat (1988).

\section{B.1 Defining UnCERTAinty}

Total measurement uncertainty consists of two components, bias uncertainty (B) and precision uncertainty $(\mathrm{P})$. Bias uncertainty is error that is consistent from measurement to measurement and is typically a function of errors in calibration of transducers and other measurement devices. Precision or random uncertainty is associated with run-to-run variation in measurements. The total uncertainty $(\Delta)$ is determined by:

$$
\Delta=\sqrt{\mathrm{B}^{2}+\mathrm{P}^{2}}
$$

Many of the bias uncertainties of measurements were estimated based on typical values for transducers, thermocouples, etc, or based on results reported in previous research using similar transducers and measurement techniques. 


\section{B.2 Determining UnCertainty of Measurements and Calculated VALUES}

\section{Uncertainty in Heat Transfer Coefficients (h)}

The bias uncertainty for the measurements of heat transfer coefficients is a result of the calibrated sensitivity of each sensor. The bias uncertainty for the heat flux sensors was detailed in Smith, et al. (1999) and in Popp (2000). The average uncertainty in the heat flux sensors, based on convection and radiation heat flux tests is estimated to be approximately $\pm 6 \%$. Since the calculation of heat transfer coefficient is determined from the slope of a plot of heat flux (q) versus $T_{t}-T_{w}$, (see section 3.2.2) any uncertainty in thermocouple measurements can only shift the curve, not alter the slope (refer to Figure $3.5 b)$. The bias uncertainty of the heat flux sensors then directly translates into the error in heat transfer coefficient.

The precision uncertainty in $\mathrm{h}$ was determined through repeated measurements using a $90 \%$ confidence interval assuming a Student-t distribution. The precision uncertainty for each location was determined by:

$$
\mathrm{P}=\frac{\Delta \mathrm{h}}{\mathrm{h}}=\frac{\mathrm{t} \cdot \mathrm{S}_{\mathrm{h}}}{\overline{\mathrm{h}} \cdot \sqrt{\mathrm{N}}}
$$

where $S_{h}$ is the variance of the heat transfer coefficient, $\overline{\mathrm{h}}$ is the mean of the heat transfer coefficient measurements, $\mathrm{N}$ is the number of samples and $\mathrm{t}$ is the Student- $\mathrm{t}$ variable.

The low turbulence precision uncertainty was determined from $\mathrm{N}=6$ separate tests $(\mathrm{t}=2.015)$ and the high turbulence heat transfer coefficient precision uncertainty was determined from $\mathrm{N}=4$ separate tests $(\mathrm{t}=2.353)$ for each gage location. Table B.1 below details the precision uncertainty determined for each gage location.

Table B.1 Precision uncertainty (P) of heat transfer coefficient tests

\begin{tabular}{|c|c|c|}
\hline Gage Location & Low Turbulence & High Turbulence \\
\hline SS1 & $1.8 \%$ & $2.3 \%$ \\
\hline PS1 & $2.8 \%$ & $1.7 \%$ \\
\hline PS2 & $1.9 \%$ & $1.7 \%$ \\
\hline
\end{tabular}


The uncertainty in measured increase in heat transfer coefficient due to turbulence is only a function of the precision uncertainties in Table B.1 since both low and high turbulence measurements are affected by the same bias uncertainty.

From equation B.1 and the precision uncertainties in Table B.1 and using a bias uncertainty of $\pm 6 \%$ for each gage location, the total uncertainty for each measurement location for both low and high turbulence heat transfer coefficients was calculated and is presented in Table B.2.

Table B.2 Total uncertainty $(\Delta)$ of heat transfer coefficient tests

\begin{tabular}{|c|c|c|}
\hline Gage Location & Low Turbulence & High Turbulence \\
\hline SS1 & $6.3 \%$ & $6.4 \%$ \\
\hline PS1 & $6.6 \%$ & $6.2 \%$ \\
\hline PS2 & $6.3 \%$ & $6.2 \%$ \\
\hline
\end{tabular}

\section{Uncertainty in hot-wire velocity and turbulence measurements}

Uncertainty in measurements of unsteady velocity is of primary concern in the form of the RMS of fluctuating velocity ( $\left.\mathrm{u}_{\mathrm{rms}}\right)$. The RMS of velocity is used both in determination of the turbulence level as well as in the analytical model calculations. Bias uncertainty for the hot-wire measurements is a function of the hot-wire calibration and the variables on which the calibration is dependent. The bias uncertainty was determined by the perturbation method outlined in Moffat (1988). Briefly, the perturbation method combines the effects on the dependent variable (quantity of interest) of perturbing the values of the independent variables by their respective uncertainties. For the measurement of hot-wire velocity, this can be presented by:

$$
\Delta \widetilde{\mathrm{u}}=\sqrt{\sum\left(\frac{\partial \widetilde{\mathrm{u}}}{\partial \mathrm{x}_{\mathrm{i}}} \cdot \Delta \mathrm{x}_{\mathrm{i}}\right)^{2}}
$$

where for the hot-wire velocity calibration, $x_{i} \in\left\{T_{t}, T_{h w}, P_{t}, P_{s}, V\right\}$, where the variables are the total temperature of the flow, the temperature of the hot-wire, the freestream total 
and static pressures and the hot-wire voltage. The hot-wire calibration technique is detailed in Nix, et al. (2002) in Appendix A. These variables were perturbed by values consistent with uncertainty for each of the transducers used to measure the values $\left( \pm 1^{\circ} \mathrm{C}\right.$ for thermocouple measurements and \pm 0.15 psi for pressure transducers) and an estimate of the hot-wire voltage uncertainty of $\pm 2 \mathrm{mV}$. The perturbation analysis was performed by the computerized method presented in Moffat (1988) using a spreadsheet. The bias uncertainty in hot-wire velocity was determined to be $\pm 2.3 \%$.

Precision uncertainty of $\mathrm{u}^{\prime}{ }_{\mathrm{rms}}$ was determined in a similar fashion as for the steady heat transfer coefficients, using a 90\% confidence level assuming a Student-t distribution:

$$
\mathrm{P}=\frac{\Delta \mathrm{u}_{\mathrm{rms}}^{\prime}}{\mathrm{u}_{\mathrm{rms}}^{\prime}}=\frac{\mathrm{t} \cdot \mathrm{S}_{\mathrm{u}^{\prime}}}{\mathrm{u}_{\mathrm{rms}}^{\prime} \cdot \sqrt{\mathrm{N}}}
$$

The precision uncertainties were determined from several repeated measurements at each measurement location. The results of equation B.4 were combined for all measurements and the precision uncertainty was determined to be $\pm 4.4 \%$. From equation B.1, the total uncertainty in the measurement of $\mathrm{u}_{\mathrm{rms}}^{\prime}$ was determined to be $\pm 5 \%$. Through perturbation analysis, the total uncertainty in the measurement of turbulence intensity was determined to be $\pm 5.5 \%$.

\section{Uncertainty in integral length scale $\left(\Lambda_{x}\right)$}

The integral length scale was determined by integrating the autocorrelation of streamwise velocity to determine the integral time-scale and then invoking Taylor's hypothesis and multiplying the integral time scale by the mean velocity. Error in the integral time scale is a function of determining the zero crossing of the autocorrelation function. Perturbation of the integral time scale was performed based on a typical autocorrelation curve and the uncertainty in integrating the autocorrelation was estimated to be about $\pm 2 \%$. The uncertainty associated with invoking Taylor's hypothesis and

multiplying by the mean velocity was estimated by combining the uncertainty in the integral time scale $( \pm 2 \%)$ and the mean velocity $( \pm 2.3 \%)$ through perturbation analysis. The bias uncertainty was then estimated to be $\pm 3 \%$ 
Precision uncertainty in calculating the integral length scale was determined from repeated measurements of the integral length scale at each gage location. The precision uncertainty was determined to be $\pm 5.3 \%$. From equation B.1, the total uncertainty in the measurement of $\Lambda_{\mathrm{x}}$ was determined to be $\pm 6.1 \%$.

\section{Uncertainty analytical model predicted heat transfer increase $\left(\Delta \mathbf{h}_{\mathbf{t}}\right)$}

The final uncertainty to be quantified is the increase in heat transfer coefficient predicted by the analytical model $\left(\Delta \mathrm{h}_{\mathrm{t}}\right)$, which is a function of both $\mathrm{u}_{\mathrm{rms}}^{\prime}$ and $\Lambda_{\mathrm{x}}$ (neglecting small uncertainty in determining the thermal conductivity (k) and thermal diffusivity $(\alpha)$ ). The bias uncertainty in the model predictions was determined through perturbation analysis using the total uncertainty calculated for the RMS of velocity fluctuations ( $\mathrm{u}_{\mathrm{rms}}^{\prime}$ ) of $\pm 5 \%$ and the integral length scale $\left(\Lambda_{\mathrm{x}}\right)$ of $\pm 6.1 \%$. The bias uncertainty on $\Delta \mathrm{h}_{\mathrm{t}}$ was determined to be $\pm 4 \%$.

Precision uncertainty in quantifying the value of $\Delta \mathrm{h}_{\mathrm{t}}$ was determined from repeated measurements at each gage location. The precision uncertainty was determined to be $\pm 2 \%$. From equation B. 1 , the total uncertainty in calculating $\Delta \mathrm{h}_{\mathrm{t}}$ was determined to be $\pm 4.5 \%$.

\section{B.3 UNCERTAINTY SUMMARY}

Table B.3 below summarizes the total uncertainty determined for all relevant parameters in this work.

Table B.3 Summary of total uncertainty $(\Delta)$

\begin{tabular}{|c|c|}
\hline Parameter & Total Uncertainty \\
\hline $\mathbf{h}$ & $6.3 \%$ \\
(average of low and high turbulence) & \\
\hline $\mathbf{u}^{\prime}{ }_{\text {rms }}$ & $5.0 \%$ \\
\hline$T u$ & $5.5 \%$ \\
\hline$\Lambda_{\mathbf{x}}$ & $6.1 \%$ \\
\hline$\Delta \mathbf{h}_{\mathbf{t}}$ & $4.5 \%$ \\
\hline
\end{tabular}




\section{APPENDIX C}

\section{COMPUTER CODE}

The following code is a Matlab M-file which was used to reduce hotwire voltage data based on recent blowdown calibration and perform analyses in the time domain and frequency domain, as well as performing analytical model analyses in both the steady and unsteady form:

\section{HWDATA062401.M:}

\% Matlab M-file to reduce Hotwire Data Using 06/22 Auspex Wire Blowdown Calibration

clear all; close all;

\%Sampling Parameters:

fsample $=100000 ; \%$ Sampling frequency of $100 \mathrm{kHz}$

$\mathrm{dt}=1 /$ fsample; \% Sampling period (sec)

$\mathrm{N}=131069 ; \%$ Number of Samples

$\mathrm{T}=\mathrm{N} * \mathrm{dt} ; \%$ Total Sampling Period (seconds)

$\mathrm{df}=1 / \mathrm{T} ; \%$ Frequency Resolution

$\mathrm{t}=[0: \mathrm{dt}: \mathrm{T}] ; \%$ Define time $\mathrm{T}=($ Number Samples $) *(\mathrm{dt}), \mathrm{dt}=1 /($ Sampling Frequency)

$\mathrm{f}=[0: \mathrm{df}$ :fsample $] ; \%$ Define frequency range from 0 to fsample in increments of $\mathrm{df}$

Patm=94760/6894.8;

Twire $=464$;

Dwire $=5 \mathrm{E}-6$;

\% DATA REDUCTION USING BLOWDOWN CALIBRATION

$\%$ Read in hotwire data from LeCroy:

load hw062401.asc; \%Read hotwire voltage

$\mathrm{V} 2=\mathrm{hw} 062401$;

$\mathrm{V} 2=\mathrm{V} 2+6 ; \%$ Add on $6 \mathrm{~V}$ offset

$\%$ Mach2 $=0.5 ; \%$ From Mach number distribution for SS2

Mach2 $=0.25 ; \%$ From Mach number distribution for PS1

$\%$ Mach2 $=0.3 ; \%$ From Mach number distribution for PS3 
Pt2 $=9.527068893 ; \%$ From low speed data

Ps2 $=(\mathrm{Pt} 2+\mathrm{Patm}) /\left(\left(1+0.2^{*} \mathrm{Mach} 2^{\wedge} 2\right)^{\wedge} 3.5\right)-\mathrm{Patm} ;$

$\mathrm{Tt} 2=299.4831878 ; \%$ From low speed data

$\mathrm{Ts} 2=\mathrm{Tt} 2 /\left(1+0.2^{*} \mathrm{Mach}^{\wedge} 2\right)$;

$\mathrm{Tm} 2=($ Ts $2+$ Twire $) / 2$;

rho $2=($ Ps $2+$ Patm $) * 6894.8 /(287 *$ Tm 2$)$;

$\mathrm{k} 2=0.02414 *(\operatorname{Tm} 2 / 273.16)^{\wedge} 1.5 *(473.16 /(\operatorname{Tm} 2+200))$;

$\mathrm{Nu} 2=\mathrm{V} 2 .^{\wedge} 2 . /\left((\mathrm{Tm} 2-\mathrm{Ts} 2)^{*} \mathrm{k} 2\right){ }^{*}(\mathrm{Tm} 2 / \mathrm{Ts} 2)^{\wedge}(-0.17)$;

$\mathrm{Re} 2=(\mathrm{Nu} 2 . / 4.6267) .^{\wedge}(1 / 0.2755)$;

$\mathrm{mu} 2=0.00001716^{*}(\mathrm{Tm} 2 / 273.16)^{\wedge} 1.5 *(383.716 /(\operatorname{Tm} 2+110.556))$;

rU2=Re2.*mu2./Dwire;

$\mathrm{U} 2=\mathrm{rU} 2 . / \mathrm{rho} 2$;

$\mathrm{Vmean}(2)=\operatorname{mean}(\mathrm{U} 2)$;

$\mathrm{v} 2=\mathrm{U} 2-\mathrm{Vmean}(2)$;

fluct $2=(\operatorname{mean}(\mathrm{v} 2 . \wedge 2)) \wedge^{\wedge} 0.5$;

$\mathrm{Tu} 2=$ fluct $2 / \operatorname{Vmean}(2)$;

\%Using (rhoU)'/rhoU:

rUp2=rU2-mean(rU2);

$\mathrm{Um} 2=\operatorname{Mach} 2 *(1.4 * 287 * \mathrm{Ts} 2)^{\wedge} 0.5$;

Tup2 $=\left(\left(\operatorname{mean}\left(\operatorname{rUp} 2 .^{\wedge} 2\right)\right) .^{\wedge} 0.5\right) . /(\operatorname{rho} 2 * \operatorname{Um} 2)$;

$\%$ Spectral Data

$\%$ Calculate Statistics for each data file:

$\mathrm{V} 22=(\mathrm{v} 2) . \wedge 2 ; \% \mathrm{a}^{\wedge} 2$

$\mathrm{V} 23=(\mathrm{v} 2) . \wedge 3 ; \% \mathrm{a}^{\wedge} 3$

$\mathrm{V} 24=(\mathrm{v} 2) . \wedge 4 ; \% \mathrm{a}^{\wedge} 4$

$\%$ Calculate RMS for each file $\left(\operatorname{RMS}=\left(\operatorname{mean}\left(\mathrm{a}^{\wedge} 2\right)\right)^{\wedge} 1 / 2\right)$ :

$\mathrm{RMS} 2=(\operatorname{mean}(\mathrm{V} 22)) . \wedge .5$;

$\%$ Calculate Kurtosis for each file $\left(\operatorname{Kurtosis}=\left(\operatorname{mean}\left(\mathrm{a}^{\wedge} 4\right)\right) /\left(\left(\operatorname{mean}\left(\mathrm{a}^{\wedge} 2\right)\right)^{\wedge} 2\right)\right)$ :

$\left.\operatorname{Kurt} 2=(\operatorname{mean}(\mathrm{V} 24)) /(\operatorname{mean}(\mathrm{V} 22))^{\wedge} 2\right)$;

$\%$ Calculate Skewness for each file $\left(\right.$ Skewness $\left.=\left(\operatorname{mean}\left(\mathrm{a}^{\wedge} 3\right)\right) /\left(\operatorname{mean}\left(\mathrm{a}^{\wedge} 2\right)\right)^{\wedge} 3 / 2\right)$ :

Skew2 $=(\operatorname{mean}(\mathrm{V} 23)) /\left(\operatorname{mean}(\mathrm{V} 22) .^{\wedge} 1.5\right)$;

\%PART b): PLOT AUTOCORRELATION CURVE AND DETERMINE INTEGRAL LENGTH SCALE:

$\%$

$\%$ Calculate autocorrelation on each data file:

$\mathrm{Rv} 2=\mathrm{xcorr}\left(\mathrm{v} 2,{ }^{\prime} \operatorname{coeff}\right)$;

Rv2=Rv2(131070:262139); 
\%Plot autocorrelations:

figure(1);

$\operatorname{plot}\left(\mathrm{t}, \mathrm{Rv} 2, \mathrm{r}^{\prime}\right)$;

title('Figure 1: Autocorrelation of Velocity Signal');

xlabel('ไtau (s)'); ylabel('Rv(|tau)');

$\operatorname{axis}\left(\left[\begin{array}{llll}0 & 0.002 & -0.2 & 1.2\end{array}\right]\right)$;

grid on;

\%Perform Numerical Integration to Calculate Integral Time Scale:

$\%$ First zero crossing calculated with find command:

$\mathrm{z} 2=$ find $(\operatorname{abs}(\operatorname{Rv} 2)<0.02 \& \operatorname{abs}(\operatorname{Rv} 2)>=0)$;

$\mathrm{T} 2=\operatorname{sum}(\operatorname{Rv} 2(1: \mathrm{z} 2(1))) * \mathrm{dt} ; \% \mathrm{~T}$ is integral time scale

$\mathrm{L}(2)=\mathrm{T} 2 * \operatorname{Vmean}(2)$;

\%PART c): DETERMINE AND PLOT PROBABILITY DENSITY FUNCTION (PDF) OF DATA SETS:

$\%$

$\%$ Create PDFs for each Data file:

$\%$ Create bin file (50 bins)

$\%$ Hotwire Signal:

$\mathrm{dV} 2=((\mathrm{Vmean}(2)+3 * \mathrm{RMS} 2)-(\mathrm{Vmean}(2)-3 * \mathrm{RMS} 2)) / 50$;

$\mathrm{m} 2=[(\mathrm{Vmean}(2)-3 * \mathrm{RMS} 2): \mathrm{dV} 2:(\mathrm{Vmean}(2)+3 * \mathrm{RMS} 2)]$;

$\mathrm{N} 2=$ hist $(\mathrm{U} 2, \mathrm{~m} 2) ; \%$ Create histogram with 50 bins whose centers are defined in the vector $\mathrm{m}$

pdf2 $=\mathrm{N} 2 . /(\operatorname{sum}(\mathrm{N} 2) * \mathrm{dV} 2) ; \%$ Normalize histogram to create PDF (divide by Ntotal and dV)

\%Plot PDFs:

figure(2);

$\operatorname{bar}\left(\mathrm{m} 2, \mathrm{pdf} 2, \mathrm{r}^{\prime}\right)$;

xlabel('Hotwire Voltage (V)'); ylabel('PDF (N_i/(N_t_o_t_a_1*ไDeltaV)');

title('Figure 2: Probability Density Function for (PDF) Velocity Signal');

grid on;

\%PART d): PLOT NON_DIMENSIONAL POWER SPECTRAL DENSITY OF DATA SETS:

$\%$

\%Perform Discrete Fourier Transform of each data set:

\%Perform FFT and scaling of each data set: 


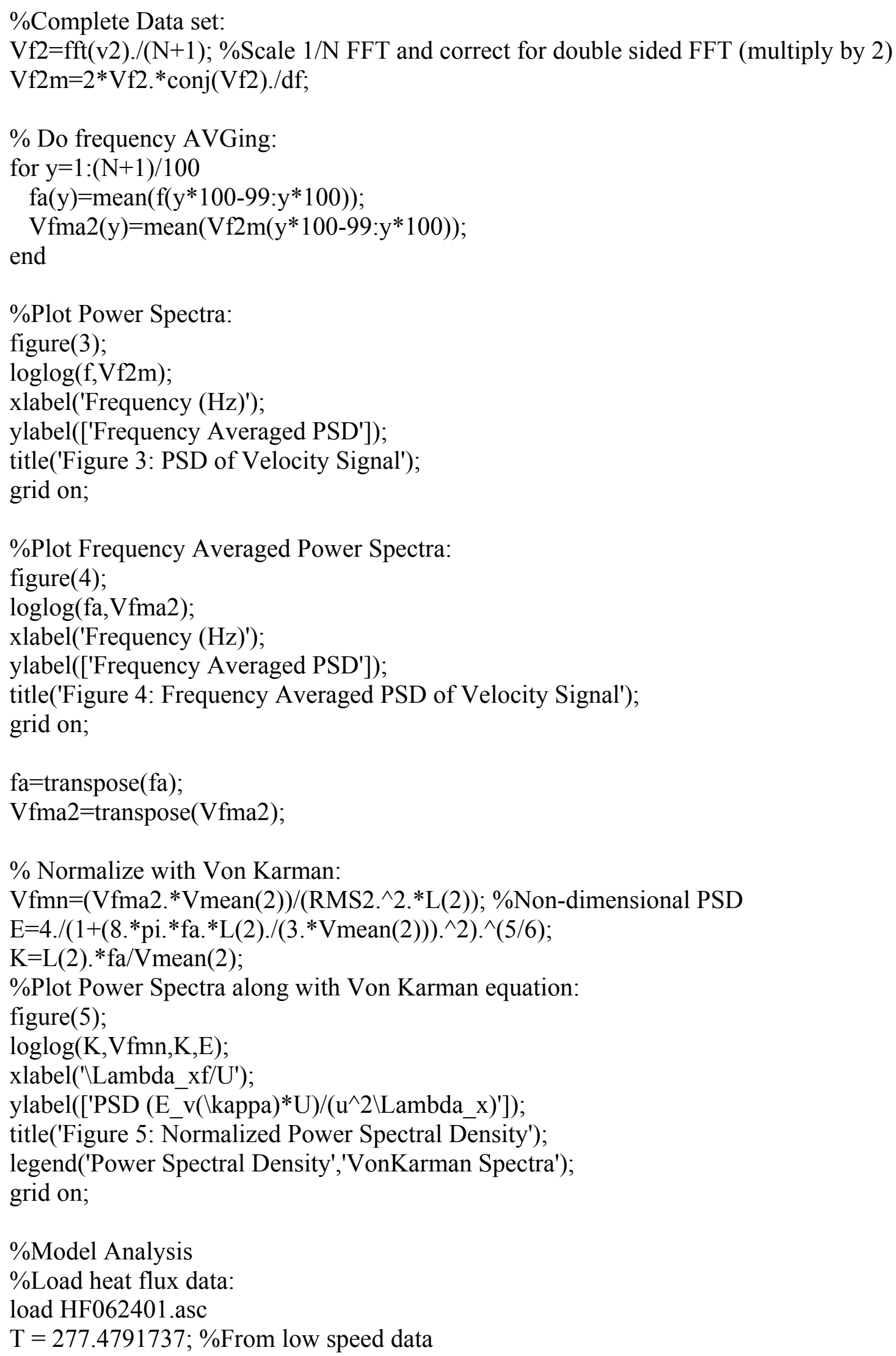


$\% \mathrm{q}=((\mathrm{HF} 062401 * 1000000) / 100) /(105) ; \%$ For SS2 location

$\mathrm{q}=((\mathrm{HF} 062401 * 1000000) / 100) /(0.236426 * \mathrm{~T}+249.4697) * 10000$; \%For $\quad$ PS1 location $\mathrm{W} / \mathrm{cm}^{\wedge} 2$

$\% \mathrm{q}=((\mathrm{HF} 062401 * 1000000) / 100) /\left(0.148635^{*} \mathrm{~T}+236.5432\right) ; \%$ For PS3 location

meanq=mean $(\mathrm{q})$;

$\mathrm{qp}=(\mathrm{q}-\mathrm{meanq})$;

\%Steady model:

urms = RMS2;

$\mathrm{Lx}=\mathrm{L}(2)$;

$\mathrm{Te}=\mathrm{Ts} 2 ; \%$ Static temp of flow

$\mathrm{ke}=0.02414^{*}(\mathrm{Te} / 273.16)^{\wedge} 1.5 *(473.16 /(\mathrm{Te}+200))$;

rho $=(\mathrm{Ps} 2+\mathrm{Patm}) * 6894.8 /(287 * \mathrm{Ts} 2)$

$\mathrm{ae}=\mathrm{ke} /(\mathrm{rho} * 1004.5)$;

qptsteady $=$ abs(ke.*(Te - T)./(pi.*ae.*Lx./urms).^0.5);

qptsteadymax $=\operatorname{abs}\left(\right.$ ke.*(Te - T)./(pi.*ae. ${ }^{*}$ Lx. $\left.\left./ \max (\mathrm{v} 2)\right) . \wedge 0.5\right)$;

dhtsteady $=$ qptsteady $/($ Ts2-T);

$\%$ Unsteady Model

$\%$ Heat Flux:

$\mathrm{Qf}=\mathrm{fft}(\mathrm{qp}) . /(\mathrm{N}+1) ; \%$ Scale $1 / \mathrm{N}$ FFT and correct for double sided FFT (multiply by 2 )

$\mathrm{Qfm}=2 * \mathrm{Qf} .{ }^{*} \operatorname{conj}(\mathrm{Qf}) . / \mathrm{df} ; \% \mathrm{PSD}$ of q fluctuations

$\%$ Determine magnitude of both velocity and heat flux FFTs:

Ufmag $=\left(\operatorname{imag}(\mathrm{Vf} 2) .^{\wedge} 2+\operatorname{real}(\mathrm{Vf} 2) \wedge^{\wedge} 2\right){ }^{\wedge} 0.5 ; \%$ Magnitude of u fluctuations in frequency domain

$\mathrm{Qfmag}=(\operatorname{imag}(\mathrm{Qf}) . \wedge 2+\operatorname{real}(\mathrm{Qf}) . \wedge 2) \wedge^{\wedge} 0.5 ; \quad \%$ Magnitude of $\mathrm{q}$ fluctuations in frequency domain

\%Apply model to Ufmag:

$\mathrm{Lxu}=(3 . * \operatorname{Vmean}(2)) . /\left(8 . *{ }^{*}\right.$ pi. $\left.{ }^{*}\right)$;

$\mathrm{Lxu}=\operatorname{transpose}(\mathrm{Lxu})$

$\mathrm{qptu}=\operatorname{abs}(\mathrm{ke} . *(\mathrm{Te}-\mathrm{T}) \cdot /($ pi.*ae.*(Lxu./Ufmag)).^0.5);

\%Apply Coherence function to data

\%coherence file determined with HFMPHW2 code from Holmberg (1996):

load next062401.coh;

fcoh $=$ next062401(1,:);

$\mathrm{fcoh}=\operatorname{transpose}(\mathrm{fcoh}(1:((\mathrm{N}+1) / 2)))$;

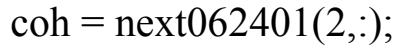

$\operatorname{coh}=\operatorname{transpose}(\operatorname{coh}(1:((\mathrm{N}+1) / 2)))$;

$\mathrm{qptcoh}=\mathrm{qptu}(1:((\mathrm{N}+1) / 2)) .{ }^{*} \mathrm{coh}$;

\%Plot measured magnitude of heat flux fluctuations vs. model with coherence:

figure(6)

$\log \log (\mathrm{f}, \mathrm{Qfmag}, \mathrm{b}$ ',fcoh,qptcoh,'r') 
xlabel('Frequency $(\mathrm{kHz})$ ');

ylabel(['Heat Flux Fluctuation Magnitude, Predicted Heat Flux (W/ $\left.\left.\left.\mathrm{cm}^{\wedge} 2\right)^{\prime}\right]\right)$;

title('Figure 6: Model Heat Flux Comparison with Magnitude of Measured Heat Flux');

axis([10e-1 40000 10e-14 1]);

legend('Measured Heat Flux Fluctuation Magnitude','Unsteady Model Results');

\%Plot measured heat flux PSD vs. model with coherence:

figure(7)

$\log \log (\mathrm{f}, \mathrm{Qfm}, \mathrm{b}$ ',fcoh,qptcoh,'r')

xlabel('Frequency $\left.(\mathrm{kHz}))^{\prime}\right)$;

ylabel(['Heat Flux Spectra, Predicted Heat Flux $\left.\left.\left(\mathrm{W} / \mathrm{cm}^{\wedge} 2\right) '\right]\right)$;

title('Figure 7: Model Heat Flux Comparison with PSD of Measured Heat Flux');

axis([10e-1 $4000010 \mathrm{e}-14$ 1]);

legend('Measured Heat Flux PSD','Unsteady Model Results');

\%Methods of combining unsteady model heat flux (section 3.4.4.3):

$\% 1$. Plot qtmeas and calculate qtrms and dqt total from measured data (no modeling):

qrms $=\operatorname{sqrt}\left(\operatorname{mean}\left(\mathrm{qp} .^{\wedge} 2\right)\right) ; \%$ RMS of measured q fluctuations calculated in time domain qrmsf $=\operatorname{sqrt}(\operatorname{sum}(\mathrm{Qfm}(1: 60000)) * \mathrm{df}) ; \% \mathrm{RMS}$ of measured q fluctuations calculated in frequency domain

dqtotal1 $=\operatorname{sqrt}\left(2 .^{*} \operatorname{sum}\left(\mathrm{Qfmag}(1: 6555) \wedge^{\wedge} 2\right)\right)$;

$\%$ Plot measured values, both PSD and Magnitude

figure (8)

$\log \log (f, \mathrm{Qfm}, \mathrm{b}$ ',f,Qfmag,'r')

xlabel('Frequency $(\mathrm{kHz})$ ');

ylabel(['PSD and Magnitude of HF Fluctuations']);

title('Figure D1: PSD and Magnitude of Fluctuations of Measured Heat Flux');

axis([10e-1 $4000010 \mathrm{e}-14$ 1]);

legend('Measured Heat Flux PSD','Magnitude of Heat Flux Fluctuations');

$\operatorname{text}\left(10,10 \mathrm{E}-10,\left(\mathrm{~W} / \mathrm{cm}^{\wedge} 2\right)^{\wedge} 2 / \mathrm{Hz}\right)$

text $\left(100,10 \mathrm{E}-10,{ }^{\prime} \mathrm{W} / \mathrm{cm}^{\wedge} 2^{\prime}\right)$

\%2. Plot qtmeas $\mathrm{x}$ coherence and calculate qtrms and dqt total from measured data (no modeling):

Qfmcoh $=$ Qfm(1:65535).*coh;

qrmsf2 $=\operatorname{sqrt}(\operatorname{sum}(\mathrm{Qfmcoh}) * \mathrm{df}) ; \%$ RMS of measured $\mathrm{q}$ fluctuations multiplied by coherence calculated in frequency domain

Qfmagcoh $=$ Qfmag(1:65535).*coh;

dqtotalcoh2 $=\operatorname{sqrt}\left(2{ }^{*} \operatorname{sum}\left(\mathrm{Qfmagcoh}(1: 6555) .{ }^{\wedge}\right)\right)$;

\% Plot measured values, both PSD and Magnitude with coherence applied: figure(9) 
$\log \log (\mathrm{fcoh}, \mathrm{Qfmcoh}, \mathrm{b}$ ',fcoh,Qfmagcoh,'r')

xlabel('Frequency $\left.(\mathrm{kHz}))^{\prime}\right)$;

ylabel(['PSD and Magnitude of HF Fluctuations']);

title('Figure D2: PSD and Magnitude of Fluctuations of Measured Heat Flux Multiplied by Coherence'); axis([10e-1 $4000010 \mathrm{e}-14$ 1]);

legend('Measured Heat Flux PSD','Magnitude of Heat Flux Fluctuations'); text $\left(10,10 \mathrm{E}-10,{ }^{\prime}\left(\mathrm{W} / \mathrm{cm}^{\wedge} 2\right)^{\wedge} 2 / \mathrm{Hz}^{\prime}\right)$ $\operatorname{text}\left(100,10 \mathrm{E}-10,{ }^{\prime} \mathrm{W} / \mathrm{cm}^{\wedge} 2^{\prime}\right)$

\%3. Plot model predicted dqt and calculate qtrms and dqt total from modeled data:

qpturms $3=\operatorname{sqrt}(\operatorname{sum}(2 . * \operatorname{qptu}(1: 60000) . \wedge 2)) ; \%$ Modeled heat flux - all frequencies dqtotal3 $=\operatorname{sqrt}\left(\operatorname{sum}\left(2 .{ }^{*} q p t u(1: 6555) \wedge^{\wedge} 2\right)\right) ; \%$ Modeled heat flux out to $5 \mathrm{kHz}$

$\%$ Plot modeled values

figure(10)

$\log \log (f, q p t u, ' b ')$

xlabel('Frequency $\left.(\mathrm{kHz}))^{\prime}\right)$; ylabel(['Modeled Heat Flux $\left.\left.\left(\mathrm{W} / \mathrm{m}^{\wedge} 2\right)^{\prime}\right]\right)$; title('Figure D3: Modeled Heat Flux'); axis([10e-1 $4000010 \mathrm{e}-41])$;

$\% 4$. Plot model predicted dqt and calculate qtrms and dqt total from modeled data with coherence:

qpturms $4=\operatorname{sqrt}(2 . * \operatorname{sum}(q p t c o h(1: 60000) . \wedge 2)) ; \%$ Modeled heat flux - all frequencies $\operatorname{dqtotal} 4=\operatorname{sqrt}\left(2 .{ }^{*} \operatorname{sum}\left(q p t c o h(1: 6555) .^{\wedge} 2\right)\right) ; \%$ Modeled heat flux out to $5 \mathrm{kHz}$

$\%$ Plot modeled values with coherence

figure(11)

$\log \log ($ fcoh,qptcoh, 'b')

xlabel('Frequency (kHz)');

ylabel(['Modeled Heat Flux x \gamma_u_q^2 (W/cm^2)']);

title('Figure D4: Modeled Heat Flux x \gamma_u_q^2');

axis([10e-1 40000 10e-14 1]);

figure(12)

$\log \log (\mathrm{f}, \mathrm{qptu}, \mathrm{b}$ ',fcoh,qptcoh,'r')

xlabel('Frequency $(\mathrm{kHz})$ ');

ylabel(['Modeled Heat Flux (W/ $\left.\left.\left.\mathrm{cm}^{\wedge} 2\right)^{\prime}\right]\right)$;

title('Figure D5: Comparison of Modeled Heat Flux With and Without Coherence');

axis([10e-1 $4000010 \mathrm{e}-14$ 1]);

legend('without Coherence','with Coherence');

\%5. Steady model using u'rms and Lx: 
figure(13)

$\log \log (f, U f m a g, ' b ', f, V f 2 m, ' r ')$

xlabel('Frequency $(\mathrm{kHz})$ ');

ylabel(['Magnitude of $u$ and PSD of $\left.u^{\prime}\right]$ );

title('Figure D6: Magnitude and PSD of Measured Velocity Fluctuations');

axis([10e-1 $4000010 \mathrm{e}-14$ 1]);

legend('Magnitude of u','PSD of u');

\%6. Similar to steady model using $\mathrm{u}^{\prime} / \mathrm{Lx}$ :

$\mathrm{uLxu}=\mathrm{Ufmag} . / \mathrm{Lxu}$;

uLxurms $\left.6=\operatorname{sqrt}\left(\operatorname{sum}(2 . * u \operatorname{Lxu}(1: 60000))^{\wedge} 2\right)\right) ; \%$ All frequencies

qpturms $6=\operatorname{abs}\left(\mathrm{ke} .{ }^{*}(\mathrm{Te}-\mathrm{T}) . /\left(\mathrm{pi} .{ }^{*} \mathrm{ae}\right){ }^{\wedge} 0.5\right) .{ }^{*} \operatorname{sqrt}(\mathrm{uLxurms} 6)$;

uLxusum $6=\operatorname{sqrt}\left(\operatorname{sum}\left(2{ }^{*} \operatorname{uLxu}(1: 6555) .{ }^{\wedge} 2\right)\right) ; \%$ Out to $5 \mathrm{kHz}$

qptusum6 $=\operatorname{abs}\left(\right.$ ke. $*($ Te $-\mathrm{T}) . /\left(\right.$ pi. ${ }^{*}$ ae $\left.) .{ }^{\wedge} 0.5\right) .{ }^{*} \operatorname{sqrt}($ uLxusum6);

\%6. Plot $\mathrm{u}^{\prime} / \mathrm{Lxu}$ values:

figure(14)

$\log \log (f, u L x u, ' b ')$

xlabel('Frequency $(\mathrm{kHz})$ ');

ylabel(['u/Lx (1/s)']);

title('Figure D7: Plot of umag/Lxu versus Frequency');

axis([10e-1 $4000010 \mathrm{e}-14$ 1]);

\%6. Similar to steady model using $\mathrm{u}^{\prime} / \mathrm{Lx}$ with Coherence:

$\mathrm{uLxucoh}=\mathrm{uLxu}(1: 65535) .{ }^{*} \mathrm{coh}$;

uLxurms $7=\operatorname{sqrt}\left(\operatorname{sum}\left(2{ }^{*} u \operatorname{uxucoh}(1: 60000) .^{\wedge} 2\right)\right) ; \%$ All frequencies

qpturms $7=\operatorname{abs}\left(\right.$ ke. ${ }^{*}(\mathrm{Te}-\mathrm{T}) . /($ pi.*ae).^0.5).*sqrt(uLxurms7);

uLxusum7 $=\operatorname{sqrt}\left(\operatorname{sum}\left(2 .{ }^{*} u \operatorname{Luxcoh}(1: 6555) . \wedge 2\right)\right) ; \%$ Out to $5 \mathrm{kHz}$

qptusum7 $=\operatorname{abs}\left(\right.$ ke. ${ }^{*}(\mathrm{Te}-\mathrm{T}) . /\left(\right.$ pi. ${ }^{*}$ ae).^0.5). ${ }^{*} \operatorname{sqrt}($ uLxusum 7$)$;

\%6. Plot u'/Lxu values with coherence:

figure(15)

$\log \log ($ fcoh,uLxucoh,'b')

xlabel('Frequency $\left.(\mathrm{kHz}))^{\prime}\right)$;

ylabel(['u/Lx x \gamma_u_q^2 (1/s)']);

title('Figure D8: Plot of umag/Lxu x \gamma_u_q^2 versus Frequency');

axis([10e-1 $4000010 \mathrm{e}-14$ 1]);

$\% 7$ Autocorrelation of $\mathrm{u}^{\prime}$ and calc of Lx....Not necessary, already done earlier:

$\% 8$ Autocorrelation of q' and calc of Lq:

$\%$ Calculate autocorrelation on each data file:

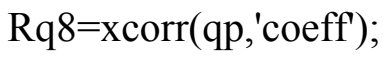

$\mathrm{Rq} 8=\mathrm{Rq} 8(131070: 262139)$;

\%Plot autocorrelations: 


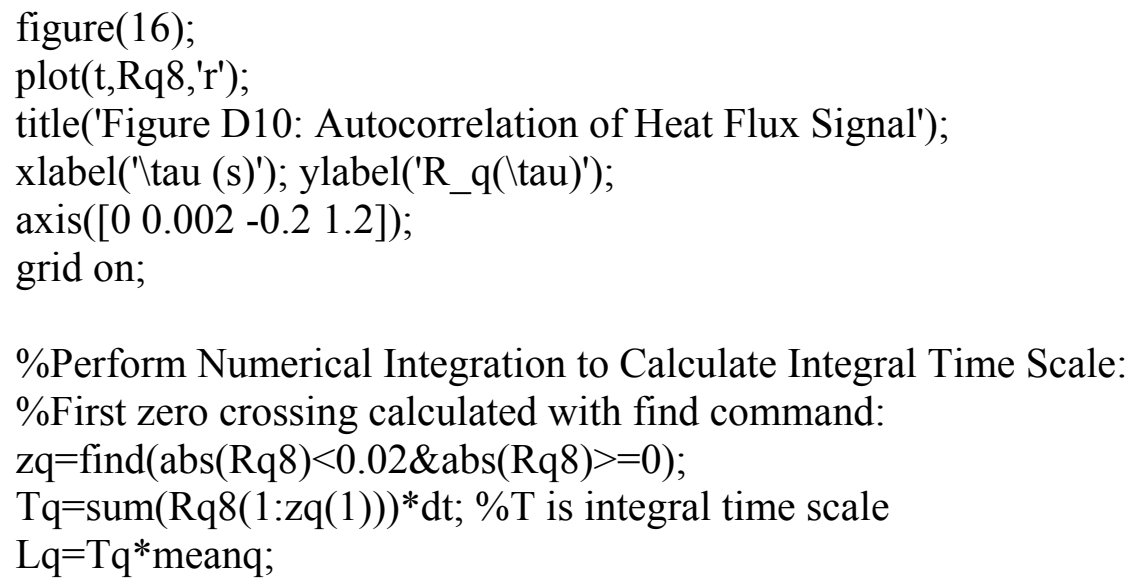




\section{VITA}

Andrew Nix was born in Ankara, Turkey in an American military hospital on July 4, 1972. He lived with his family while his father was stationed at several military assignments in Arizona, Pennsylvania, and Germany before settling in Smithsburg, Maryland where he attended middle and high school. Growing up near the Potomac River and the Blue Ridge Mountains, the author developed a love for fishing and a great appreciation for nature and the outdoors. After graduating from Smithsburg High School in June 1990, he began his undergraduate studies in Mechanical Engineering at the University of Maryland, Baltimore County in the Fall of 1990. While pursuing his BS, he worked for four years as an Atmospheric Scientist/Engineering Technician for Versar, Inc., an environmental consulting firm in Columbia, MD. He received his Bachelor of Science in Engineering from UMBC in the Spring of 1995, graduating summa cum laude. The following Fall, he began his graduate studies at the Virginia Polytechnic Institute and State University in Blacksburg, VA as a graduate research assistant for Dr. W. F. Ng and Dr. T. E. Diller studying heat transfer in gas turbines and received his MS in Mechanical Engineering in December, 1996. Upon completion of his Master's degree, he worked for

three years in the field of power generation and thermal cycle design. In 1998, he married his best friend, Cheryl Ann Gaumer. In August of 1999, he left his job at Bechtel Power Corporation in Frederick, Maryland to pursue his Ph.D at Virginia Tech. After three and a half years of research work as a Graduate Research Assistant and Research Associate studying various aspects of gas turbine heat transfer, ranging from turbulence, shock waves and film cooling, the author completed his dissertation in March of 2003 and successfully defended his Ph.D work in April. The author is pursuing a career in academia and is searching for a faculty position. 